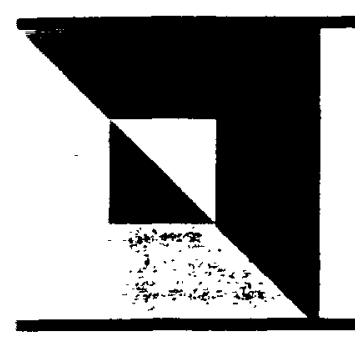

BNL-NCS-67469

Informal Report

ENDF-362

KAERI/TR-1511/2000

\title{
Neutron Cross Section Evaluations of Fission Products Below the Fast Energy Region
}

\section{April 2000}

Soo-Youl Oh and Jonghwa Chang

Nuclear Data Evaluation Laboratory

Korea Atomic Energy Research Institute

\author{
Said Mughabghab \\ National Nuclear Data Center \\ Brookhaven National Laboratory
}



BNL-NCS-67469

Informal Report

ENDF-362

KAERI/TR-1511/2000

\title{
NEUTRON CROSS SECTION EVALUATIONS OF FISSION PRODUCTS BELOW THE FAST ENERGY REGION*
}

\author{
April 2000 \\ Soo-Youl Oh and Jonghwa Chang \\ Nuclear Data Evaluation Laboratory \\ Korea Atomic Energy Research Institute \\ Said F. Mughabghab \\ National Nuclear Data Center \\ Brookhaven National Laboratory
}

*This work is supported by the U.S. Department of Energy under Contract DE-AC02-98CH10886. 


\section{DISCLAIMER}

This report was prepared as an account of work sponsored by an agency of the United States Government. Neither the United States Government nor any agency thereof, nor any of their employees, not any of their contractors, subcontractors, or their employees, makes any warranty, express or implied, or assumes any legal liability or responsibility for the accuracy, completeness, or usefulness of any information, apparatus, product, or process disclosed, or represents that its use would not infringe privately owned rights. Reference herein to any specific commercial product, process, or service by trade name trademark, manufacturer, or otherwise, does not necessarily constitute or imply its endorsement, recommendation, or favoring by the United States Government or any agency, contractor, or subcontractor thereof. The views and opinions of authors expressed herein do not necessarily state or reflect those of the United States Government or any agency, contractor or subcontractor thereof. 


\section{ABSTRACT}

Neutron cross section evaluations of the fission-product isotopes, ${ }^{95} \mathrm{Mo},{ }^{99} \mathrm{Tc},{ }^{101} \mathrm{Ru}$, ${ }^{103} \mathrm{Rh},{ }^{105} \mathrm{Pd},{ }^{109} \mathrm{Ag},{ }^{131} \mathrm{Xe},{ }^{133} \mathrm{Cs},{ }^{141} \mathrm{Pr},{ }^{141} \mathrm{Nd},{ }^{147} \mathrm{Sm},{ }^{149} \mathrm{Sm},{ }^{150} \mathrm{Sm},{ }^{151} \mathrm{Sm},{ }^{152} \mathrm{Sm},{ }^{153} \mathrm{Eu},{ }^{155} \mathrm{Gd}$, and ${ }^{157} \mathrm{Gd}$ were carried out below the fast neutron energy region within the framework of the BNL-KAERI international collaboration. In the thermal energy region, the energy dependence of the various cross-sections was calculated by applying the multi-level Breit-Wigner formalism. In particular, the strong energy dependence of the coherent scattering lengths of ${ }^{155} \mathrm{Gd}$ and ${ }^{157} \mathrm{Gd}$ were determined and were compared with recent calculations of Lynn and Seeger. In the resonance region, the recommended resonance parameters, reported in the BNL compilation, were updated by considering resonance parameter information published in the literature since 1981. The s-wave and, if available, p-wave reduced neutron widths were analyzed in terms of the Porter-Thomas distribution to determine the average level spacings and the neutron strength functions. Average radiative widths were also calculated from measured values of resolved energy resonances. The average resonance parameters determined in this study were compared with those in the BNL and other compilations, as well as the ENDF/B-VI, JEF-2.2, and JENDL3.2 data libraries. The unresolved capture cross sections of these isotopes, computed with the determined average resonance parameters, were compared with measurements, as well as the ENDF/B-VI evaluations. To achieve agreement with the measurements, in a few cases minor adjustments in the average resonance parameters were made.

Because of astrophysical interest, the Maxwellian capture cross sections of these nuclides at a neutron temperature of $30 \mathrm{keV}$ were computed and were compared with other compilations and evaluations. 



\section{ACKNOWLEDGEMENTS}

The authors gratefully acknowledge the financial support of the Ministry of Science and Technology, Korea. The cooperation of the U.S. Department of Energy in the preparation of this publication is acknowledged. We also wish to thank Alexsandra Lopez for her secretarial help in the preparation of this manuscript. 



\section{TABLE OF CONTENTS}

$\underline{\text { Page }}$

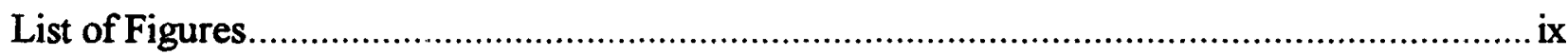

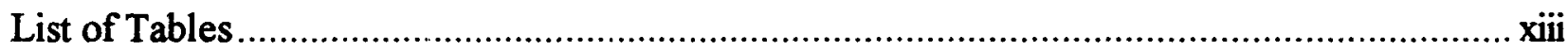

I. INTRODUCTION

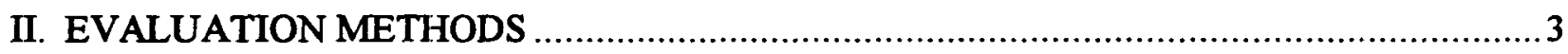

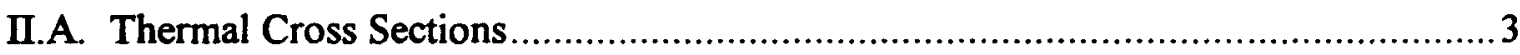

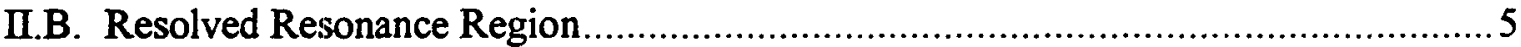

II.C. Unresolved Resonance Region .................................................................

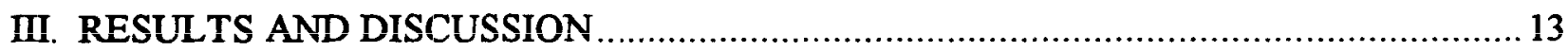

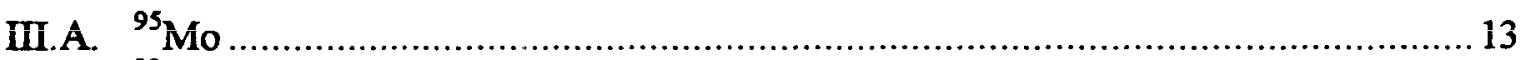

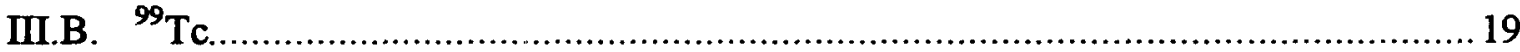

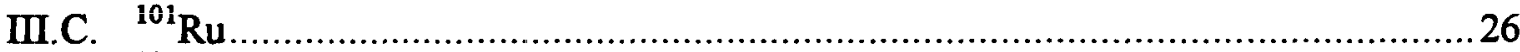

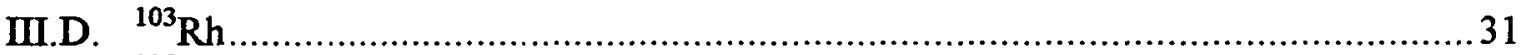

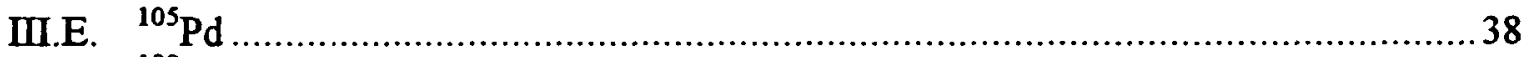

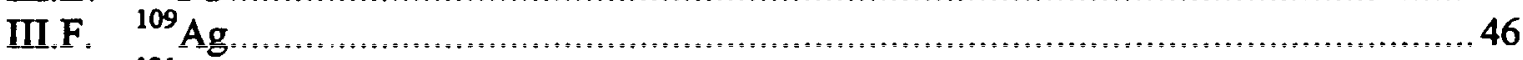

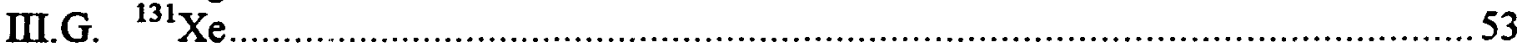

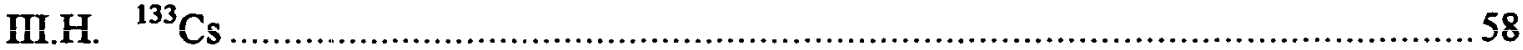

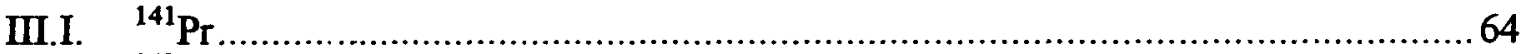

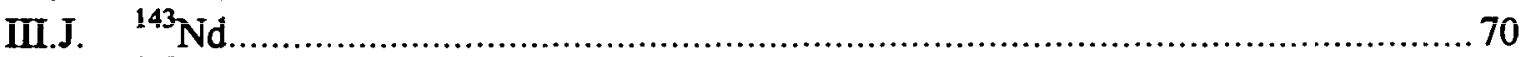

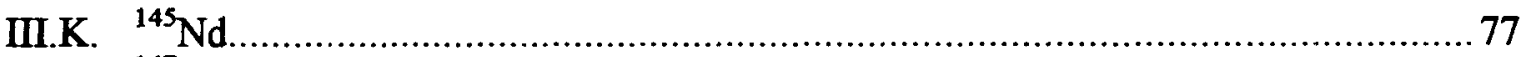

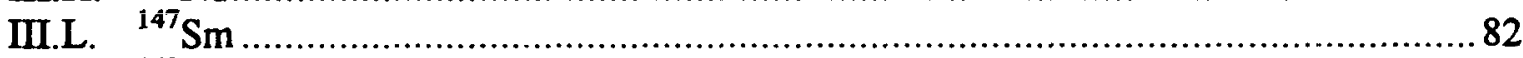

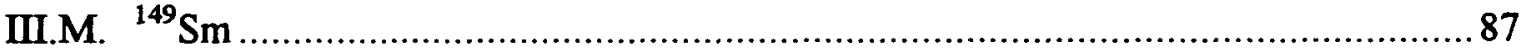

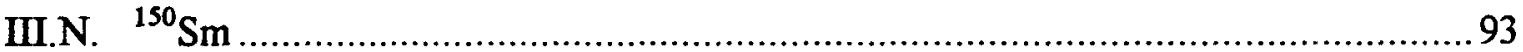

III.O. ${ }^{151} \mathrm{Sm}$

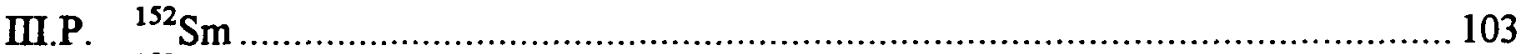

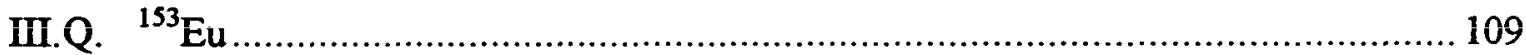

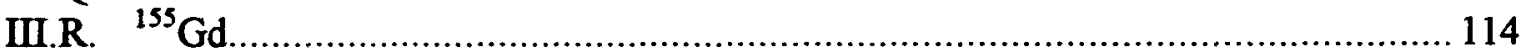

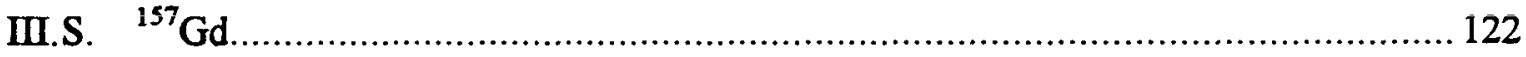

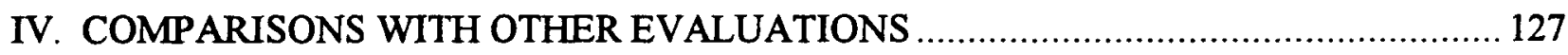

IV.A. Resolved and Unresolved Resonance Data Files ...................................... 127

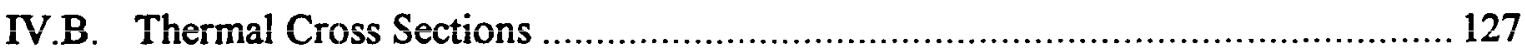

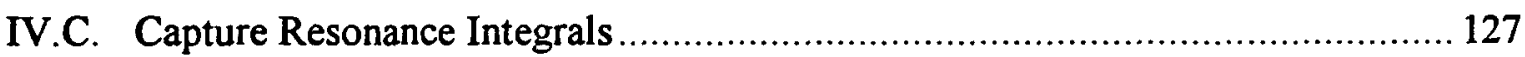

IV.D. Thermai Elastic Cross Sections .............................................................. 127

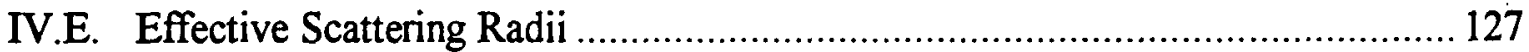




\section{TABLE OF CONTENTS (cont.)}

Page

IV.F. Wescott Factors for Capture Cross Sections ............................................... 127

IV.G. Wescott Factors for Scattering and Total Cross Sections ............................. 128

IV.H. S-Wave Strength Functions.................................................................... 128

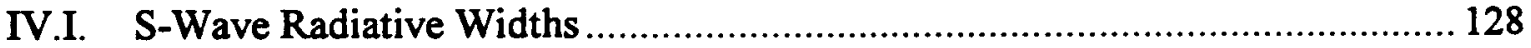

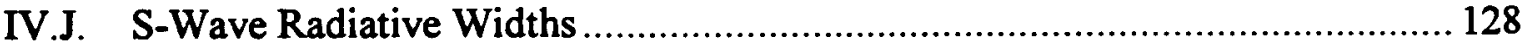

IV.K. Maxwellian Capture Cross Sections at $30 \mathrm{KeV}$......................................... 128

REFERENCES

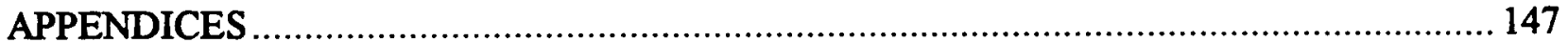




\section{List of Figures}

Page

Figure 1. Complement cdf for p-wave Neutron Reduced Widths...................................11

Figure 2. Complement of the Cumulative Distribution of Neutron

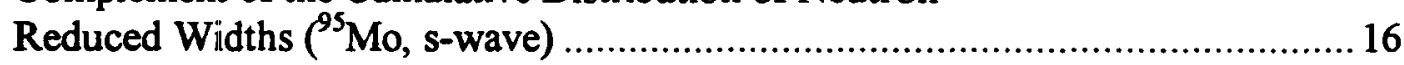

Figure 3. Complement of the Cumulative Distribution of Neutron

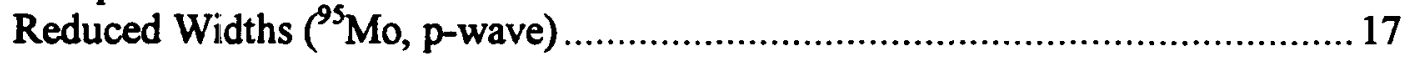

Figure 4. Capture Cross Section in the Unresolved Resonance Region $\left({ }^{95} \mathrm{Mo}\right) \ldots \ldots \ldots \ldots \ldots . . . .18$

Figure 5. Complement of the Cumulative Distribution of Neutron

Reduced Widths $\left({ }^{99} \mathrm{Tc}\right)$. All resonances were assumed as s-wave. .................... 22

Figure 6. Complement of the Cumulative Distribution of Neutron

Reduced Widths $\left({ }^{99} \mathrm{Tc}\right.$, s-wave)

Figure 7. Complement of the Cumulative Distribution of Neutron

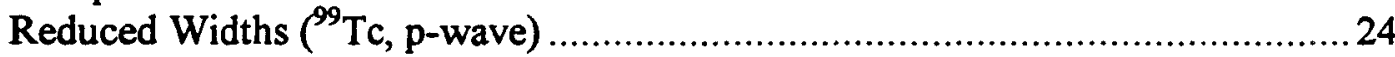

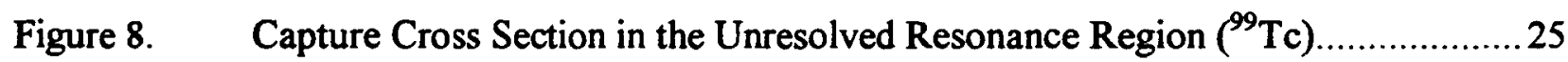

Figure 9. Complement of the Cumulative Distribution of Neutron

Reduced Widths $\left({ }^{101} \mathrm{Ru}\right.$, s-wave)

Figure 10. Capture Cross Section in the Unresolved Resonance Region $\left({ }^{101} \mathrm{Ru}\right) \ldots \ldots \ldots \ldots \ldots \ldots . . . . .30$

Figure 11. Complement of the Cumulative Distribution of Neutron

Reduced Widths $\left({ }^{103} \mathrm{Rh}\right)$. All resonances were assumed as s-wave

Figure 12. Complement of the Cumulative Distribution of Neutron

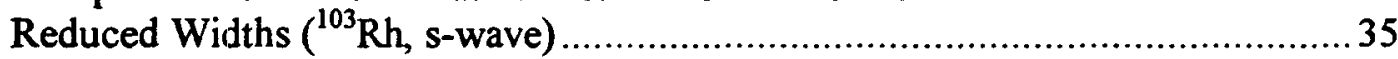

Figure 13. Complement of the Cumulative Distribution of Neutron

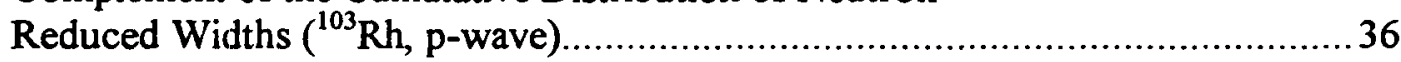

Figure 14. Capture Cross Section in the Unresolved Resonance Region $\left({ }^{103} \mathrm{Rh}\right) \ldots \ldots \ldots \ldots \ldots . . . . .37$

Figure 15. Complement of the Cumulative Distribution of Neutron

Reduced Widths ( ${ }^{105} \mathrm{Pd}$, s-wave) 


\section{List of Figures (cont.)}

Page

Figure 16. Staircase Plot of the Neutron Reduced Widths $\left({ }^{105} \mathrm{Pd}\right.$, s-wave) $\ldots \ldots \ldots \ldots \ldots \ldots \ldots \ldots \ldots . . . . . . . . \ldots 2$

Figure 17. Complement of the Cumulative Distribution of Neutron

Reduced Widths ( ${ }^{105} \mathrm{Pd}$, s-wave: fitted with the degree of freedom of 2 )

Figure 18. Complement of the Cumulative Distribution of Neutron Reduced Widths $\left({ }^{105} \mathrm{Pd}\right.$, p-wave)

Figure 19. Capture Cross Section in the Unresolved Resonance Region $\left({ }^{105} \mathrm{Pd}\right)$

Figure 20. Complement of the Cumulative Distribution of Neutron Reduced Widths $\left({ }^{109} \mathrm{Ag}\right.$, s-wave).

Figure 21. Staircase Plot of the Number of Resonances $\left({ }^{109} \mathrm{Ag}\right.$, s-wave).

The dashed line shows the data and the solid line fits linearly to the data. .50

Figure 22. Complement of the Cumulative Distribution of Neutron

Reduced Widths $\left({ }^{109} \mathrm{Ag}\right.$, p-wave)

Figure 23. Capture Cross Section in the Unresolved Resonance Region $\left({ }^{109} \mathrm{Ag}\right) \ldots \ldots \ldots \ldots \ldots \ldots . .52$

Figure 24. Complement of the Cumulative Distribution of Neutron

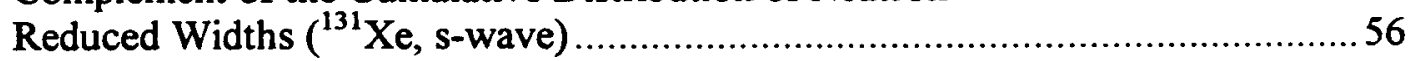

Figure 25. Capture Cross Section in the Unresolved Resonance Region $\left({ }^{131} \mathrm{Xe}\right) \ldots \ldots \ldots \ldots \ldots \ldots . . .57$

Figure 26. Complement of the Cumulative Distribution of Neutron

Reduced Widths ( ${ }^{133} \mathrm{Cs}$, s-wave)

Figure 27. Complement of the Cumulative Distribution of Neutron

Reduced Widths $\left({ }^{133} \mathrm{Cs}\right.$, p-wave)

Figure 28. Capture Cross Section in the Unresolved Resonance Region $\left({ }^{133} \mathrm{Cs}\right)$

Figure 29. Complement of the Cumulative Distribution of Neutron

Reduced Widths $\left({ }^{141} \mathrm{Pr}\right.$, s-wave)

Figure 30. Complement of the Cumulative Distribution of Neutron

Reduced Widths $\left({ }^{141} \mathrm{Pr}\right.$, p-wave) 68 


\section{List of Figures (cont.)}

Page

Figure 31. Capture Cross Section in the Unresolved Resonance Region $\left({ }^{141} \mathrm{Pr}\right)$ 69

Figure 32. Complement of the Cumulative Distribution of Neutron

Reduced Widths $\left({ }^{143} \mathrm{Nd}\right)$. All resonances were assumed as s-wave. 73

Figure 33. Complement of the Cumulative Distribution of Neutron Reduced Widths $\left({ }^{143} \mathrm{Nd}\right.$, s-wave).

Figure 34. Complement of the Cumulative Distribution of Neutron Reduced Widths $\left({ }^{143} \mathrm{Nd}\right.$, p-wave). 75

Figure 35. Capture Cross Section in the Unresolved Resonance Region $\left({ }^{143} \mathrm{Nd}\right) \ldots \ldots \ldots \ldots \ldots . . . . .76$

Figure 36. Complement of the Cumulative Distribution of Neutron Reduced Widths ( ${ }^{145} \mathrm{Nd}$, s-wave) 80

Figure 37. Capture Cross Section in the Unresolved Resonance Region $\left({ }^{145} \mathrm{Nd}\right)$....... 81

Figure 38. Complement of the Cumulative Distribution of Neutron

Reduced Widths ( ${ }^{147} \mathrm{Sm}$, s-wave) 85

Figure 39. Capture Cross Section in the Unresolved Resonance Region $\left({ }^{147} \mathrm{Sm}\right) \ldots \ldots \ldots \ldots . . . .86$

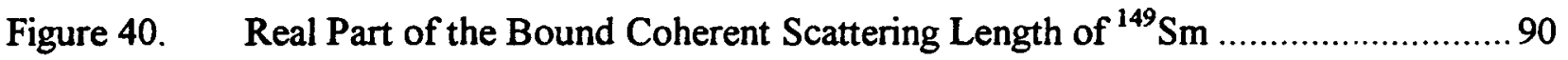

Figure 41. Complement of the Cumulative Distribution of Neutron Reduced Widths ( ${ }^{149} \mathrm{Sm}$, s-wave)

Figure 42. Capture Cross Section in the Unresolved Resonance Region $\left({ }^{149} \mathrm{Sm}\right)$

Figure 43. Complement of the Cumulative Distribution of Neutron Reduced Widths ( ${ }^{150} \mathrm{Sm}$, s-wave) 96

Figure 44. Capture Cross Section in the Unresolved Resonance Region $\left({ }^{150} \mathrm{Sm}\right)$

Figure 45. Complement of the Cumulative Distribution of Neutron Reduced Widths ( ${ }^{151} \mathrm{Sm}$, s-wave) 101

Figure 46. Capture Cross Section in the Unresolved Resonance Region $\left({ }^{151} \mathrm{Sm}\right)$ 102 


\section{List of Figures (cont.)}

Page

Figure 47. Complement of the Cumulative Distribution of Neutron

Reduced Widths ( ${ }^{152} \mathrm{Sm}$, s-wave)

Figure 48. Capture Cross Sections in the Evaluated Libraries in the

Unresolved Resonance Region $\left({ }^{152} \mathrm{Sm}\right)$

Figure 49. Capture Cross Section in the Unresolved Resonance Region $\left({ }^{152} \mathrm{Sm}\right)$ 108

Figure 50. Complement of the Cumulative Distribution of Neutron Reduced Widths ( ${ }^{153} \mathrm{Eu}$, s-wave)

Figure 51. Capture Cross Section in the Unresolved Resonance Region $\left({ }^{153} \mathrm{Eu}\right)$

Figure 52. The Total Cross Section Multiplied by the Square-root of Energy $\left({ }^{155} \mathrm{Gd}\right) \ldots \ldots . .118$

Figure 53. Real Part of the Bound Coherent Scattering Length of ${ }^{155} \mathrm{Gd}$

Figure 54. Complement of the Cumulative Distribution of Neutron

Reduced Widths ( ${ }^{155} \mathrm{Gd}$, s-wave)

Figure 55. Capture Cross Section in the Unresolved Resonance Region $\left({ }^{155} \mathrm{Gd}\right)$.

Figure 56. Bound Coherent Scattering Lengths of ${ }^{157} \mathrm{Gd}$

Figure 57. Complement of the Cumulative Distribution of Neutron Reduced Widths ( ${ }^{157} \mathrm{Gd}$, s-wave).

Figure 58. Capture Cross Section in the Unresolved Resonance Region $\left({ }^{157} \mathrm{Gd}\right)$ 126

Figure A1. Evaluation Procedure.

Figure A2. Flow of Computer Codes 150 


\section{List of Tables}

Page

Table 1. Ranking of Fission Products for a 4.5\% U-235 Fuel and 50 GWD/MTU...........2

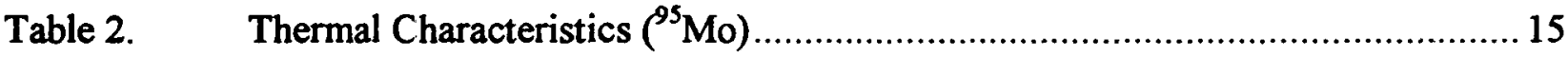

Table 3. Average Resonance Parameters for the Unresolved

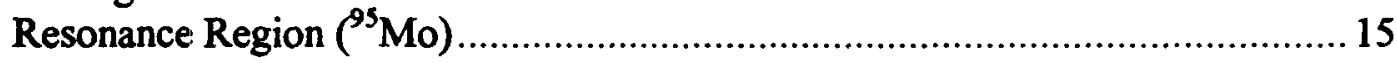

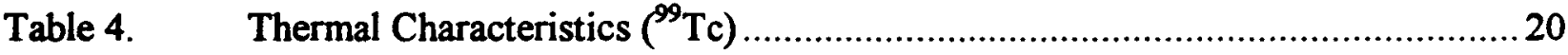

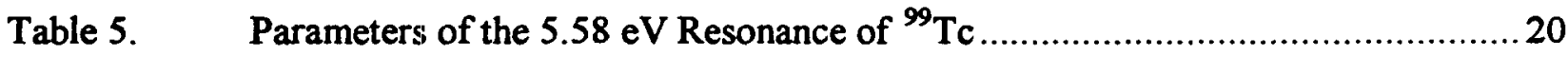

Table 6. Average Resonance Parameters for Unresolved Resonance Region $\left({ }^{99} \mathrm{Tc}\right) \ldots \ldots \ldots 21$

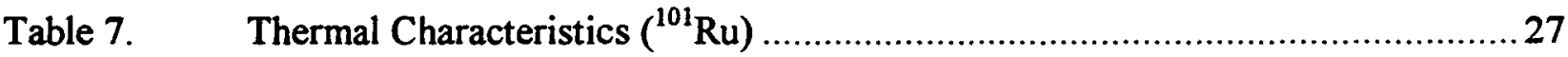

Table 8. Average Resonance Parameters for the Unresolved

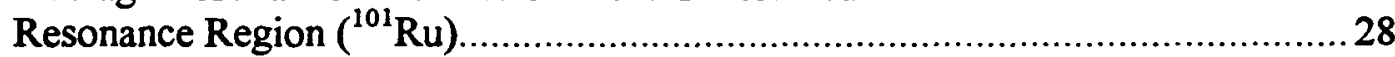

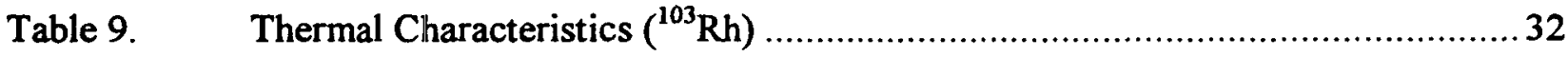

Table 10. Average Resonance Parameters in Unresolved Resonance Region $\left({ }^{103} \mathrm{Rh}\right) \ldots \ldots . . .33$

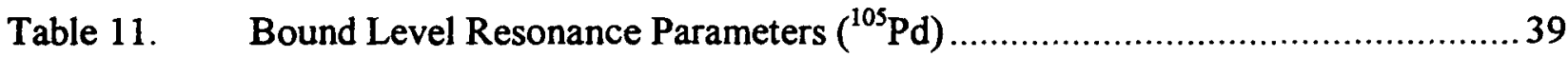

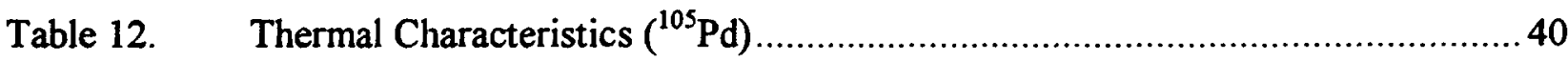

Table 13. Average Resonance Parameters for the Unresolved

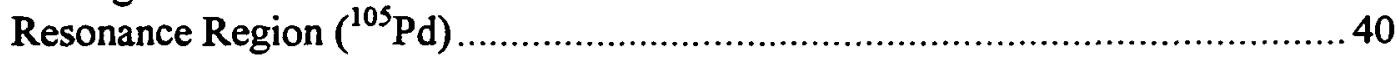

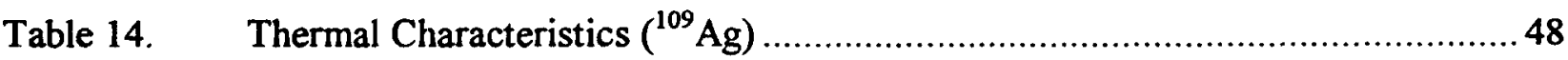

Table 15. Average Resonance Parameters for the Unresolved

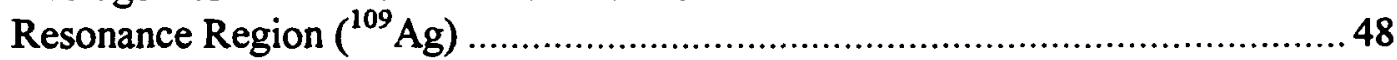

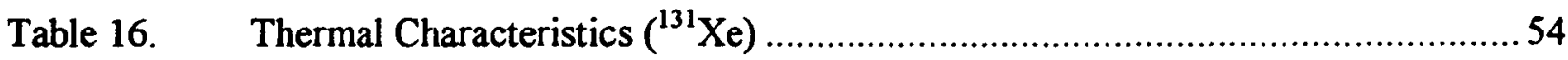

Table 17. Average Resonance Parameters for the Unresolved

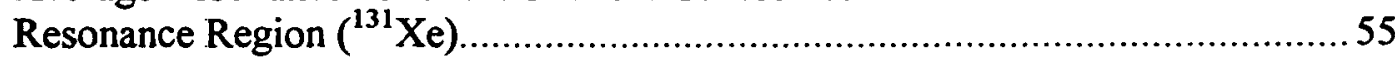




\section{List of Tables (cont.)}

$\underline{\text { Page }}$

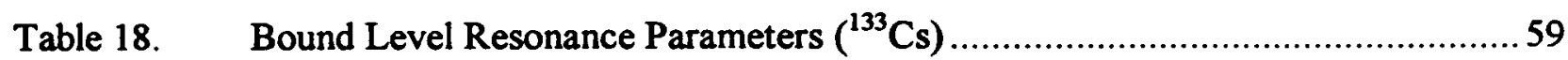

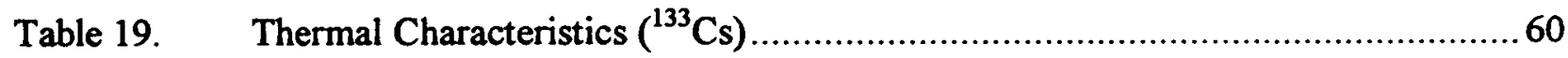

Table 20. Average Resonance Parameters for Unresolved Resonance Region $\left({ }^{133} \mathrm{Cs}\right) \ldots \ldots . .60$

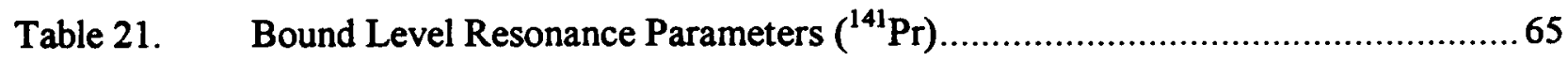

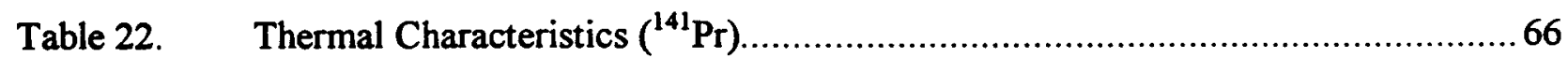

Table 23. Average Resonance Parameters for Unresolved Resonance Region $\left({ }^{141} \mathrm{Pr}\right) \ldots \ldots . .66$

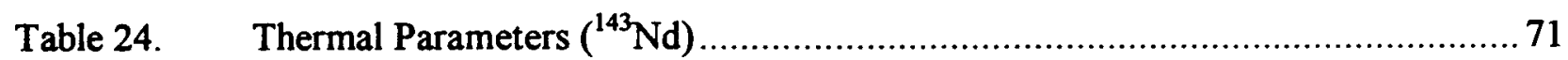

Table 25. Average Resonance Parameters in the Unresolved

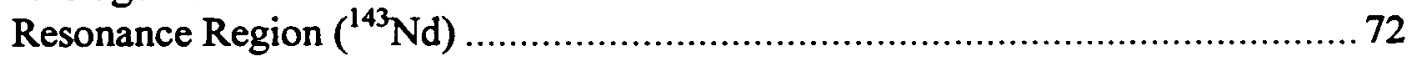

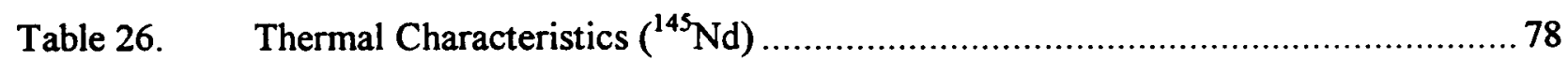

Table 27. Average Resonance Parameters in the Unresolved

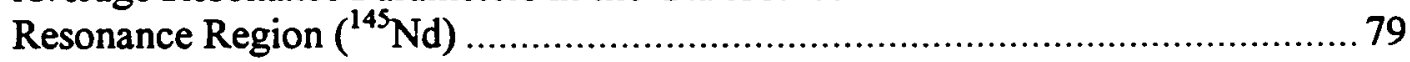

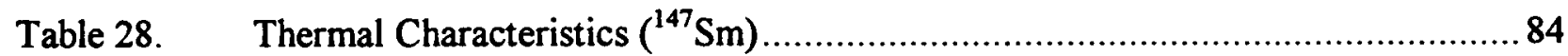

Table 29. Average Resonance Parameters for the Unresolved

Resonance Region ( ${ }^{147} \mathrm{Sm}$ ) 84

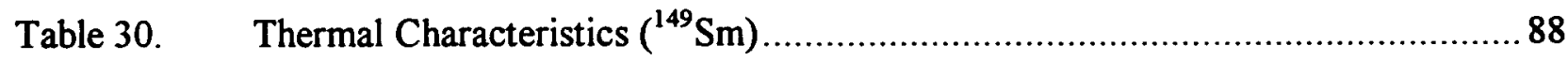

Table 31. Average Resonance Parameters in the Unresolved

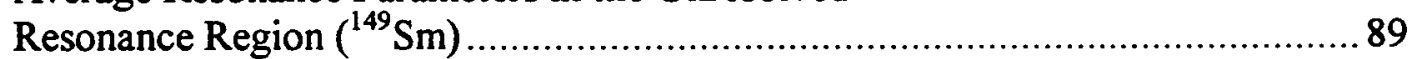

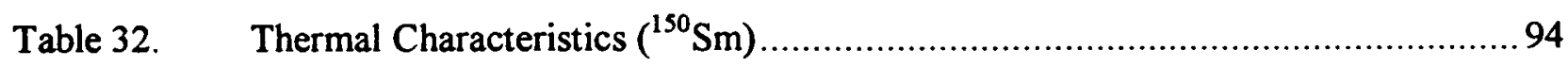

Table 33. Average Resonance Parameters for the Unresolved

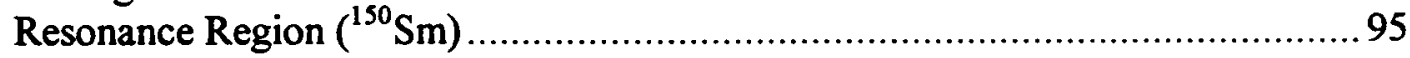

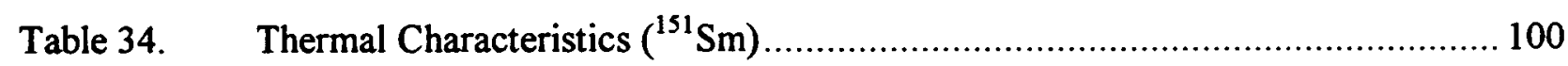

Table 35. Capture and Inelastic Scattering Cross Sections at $10 \mathrm{keV}$ of ${ }^{151} \mathrm{Sm} \ldots \ldots \ldots \ldots . . . . .100$ 


\section{List of Tables (cont.)}

Table 36. Average Resonance Parameters for the Unresolved

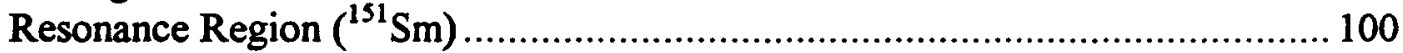

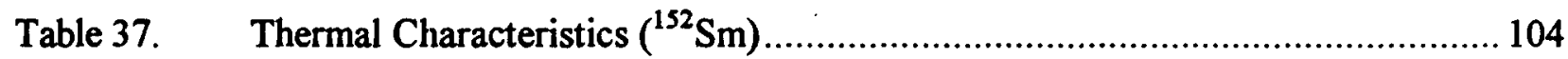

Table 38. Average Resonance Parameters for the Unresolved

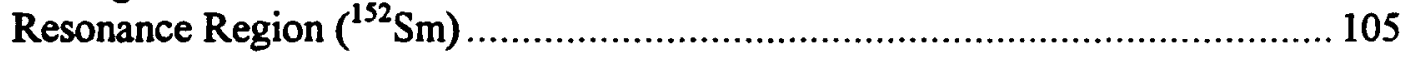

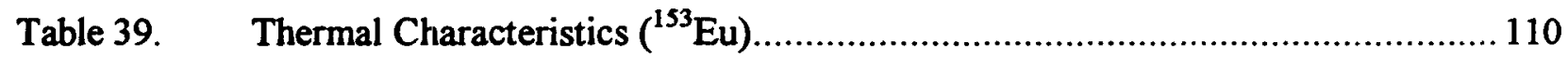

Table 40. Average Resonance Parameters for the Unresolved

Resonance Region $\left({ }^{153} \mathrm{Eu}\right)$.

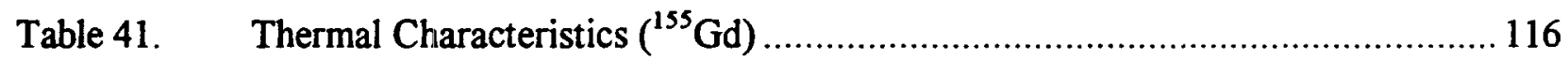

Table 42. Parameters of the First Resonance and Scattering Lengths (Real Part) of ${ }^{155} \mathrm{Gd}$.

Table 43. Average Resonance Parameters in the Unresolved

Resonance Region $\left({ }^{155} \mathrm{Gd}\right)$.

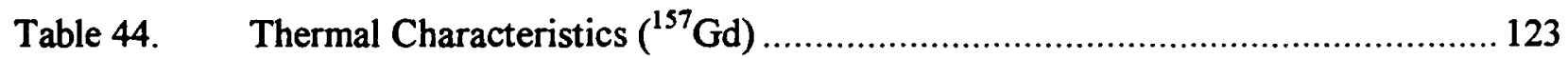

Table 45. Average Resonance Parameters for the Unresolved

Resonance Region ( $\left.{ }^{157} \mathrm{Gd}\right)$.

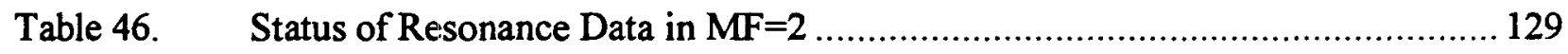

Table 47. Capture Cross Sections at $0.0253 \mathrm{eV}$ (barn) .................................... 130

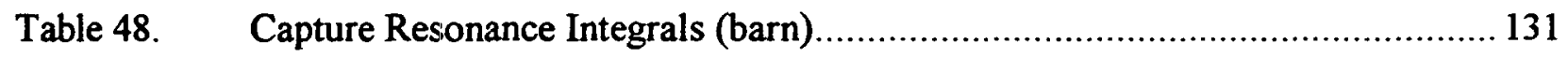

Table 49. Elastic Scattering Cross Sections at $0.0253 \mathrm{eV}$ (bam) ........................... 132

Table 50. Effective Scattering Radii (fm) ..................................................... 133

Table 51. Westcott Factors for Capture...................................................... 134

Table 52. Elastic Scattering and Total Resonance Integrals................................. 135 


\section{List of Tables (cont.)}

Page

Table 53. s-wave Neutron Strength Functions in the Unresolved

Resonance Region $\left(\times 10^{4}\right)$

Table 54. s-wave Average Level Spacings in the Unresolved

Resonance Region (eV)

Table 55. s-wave Average Radiative Widths in the Unresolved

Resonance Region (meV)

Table 56. Average Capture Cross Sections Weighted with Maxwellian

Spectrum at $30 \mathrm{keV}(\mathrm{mb})$

Table B1. Variables appearing in the PTANAL.INP file 152

Table B2. CFMTA Format (for each resonance) 153

Table C1. Variables appearing in the WRIURR.INP file 155 


\section{INTRODUCTION}

Evaluated neutron data play a major role in the design of nuclear reactors, as well as reactor safety analysis. The evaluated ENDF/B VI data libraries currently being used for applications, such as burn-up credit for spent fuel transportation, disposal criticality analysis and design of future advanced fuel do not take advantage of the recent measured cross section information. The consequence of not using the latest experimental data is likely to result in large uncertainties that have to be addressed by overly conservative assumptions or design margins.

Although the current ENDF/B-VI libraries contain data for fission product nuclides that are of importance to long term criticality or dose calculations of spent fuel, the data for many of the other fission products and higher actinides that are not major contributors to in-reactor performance may be of significant importance for criticality and dose calculations involving long-term discharge fuel. Many of such evaluations were based on rather perfunctory evaluations conducted in the 1973 to 1984 time period. Even though new measured data has become available since then, because of priority and resource considerations, these data were not re-evaluated to take advantage of the new information, but were simply copied over from earlier versions of the ENDF/B library into the current one without modification. To a certain extent, the same situation applies to the JEF-2 and JENDL-3 libraries. Table 1, which lists nineteen fission products of importance for criticality safety and transportation, gives in columns 1, 2,3, and 4 the ranking of the fission product isotope according to DeHart [De95], the fission product nuclide, the ENDF/B-VI release date, and the data source of the neutron resonance parameters, respectively.

To address the deficiency in and increase the accuracy of fission product nuclear data, a BNLKAERI collaboration was initiated in 1998. Under this project of international collaboration, the fission product nuclides listed in Table 1 were re-evaluated in the thermal, resolved resonance region, and the unresolved resonance region.

We present in Section II the evaluation methods for the thermal, resolved and unresolved energy regions; in Section III, we report the results and discussion for each fission product nuclide; in Section IV we give comparisons with other evaluations; and in Section V, we summarize the conclusions of the present evaluation project. 
Table 1. Ranking of Fission Products for a 4.5\% U-235 Fuel and 50 GWD/MTUా

\begin{tabular}{|c|c|c|c|}
\hline RANK & $\begin{array}{l}\text { FISSION } \\
\text { PRODUCT }\end{array}$ & $\begin{array}{c}\text { ENDF-VI } \\
\text { EVALUATION DATE }\end{array}$ & $\begin{array}{c}\text { DATA SOURCE OF } \\
\text { RESONANCE PARAMETERS }\end{array}$ \\
\hline 1 & ${ }^{149} \mathrm{Sm}$ & 1978 & Mughabghab (1973) \\
\hline 2 & ${ }^{143} \mathrm{Nd}$ & 1974 & Mughabghab (1984) \\
\hline 3 & ${ }^{103} \mathrm{Rh}$ & 1978 & Mughabghab (1973) \\
\hline 4 & ${ }^{151} \mathrm{Sm}$ & 1989 & Mughabghab(1984) \\
\hline 5 & ${ }^{155} \mathrm{Gd}$ & 1977 & Mughabghab (1973) \\
\hline 6 & ${ }^{131} \mathrm{Xe}$ & 1978 & Ribon (1969) \\
\hline 7 & ${ }^{133} \mathrm{Cs}$ & 1978 & Garg (1965) \\
\hline 8 & ${ }^{99} \mathrm{Tc}$ & 1978 & Mughabghab (1973) \\
\hline 9 & ${ }^{152} \mathrm{Sm}$ & 1980 & Mughabghab (1984) \\
\hline 10 & ${ }^{153} \mathrm{Eu}$ & 1986 & Mughabghab (1973) \\
\hline 11 & ${ }^{145} \mathrm{Nd}$ & 1980 & Mughabghab (1984) \\
\hline 12 & ${ }^{150} \mathrm{Sm}$ & 1980 & Mughabghab (1984) \\
\hline 13 & ${ }^{147} \mathrm{Sm}$ & 1988 & Mughabghab (1984) \\
\hline 14 & ${ }^{109} \mathrm{Ag}$ & 1983 & Mughabghab (1973) \\
\hline 15 & ${ }^{95} \mathrm{Mo}$ & 1990 & Mughabghab (1973) \\
\hline 16 & ${ }^{101} \mathrm{Ru}$ & 1980 & Mughabghab (1981) \\
\hline 17 & ${ }^{105} \mathrm{Pd}$ & 1989 & Mughabghab (1981) \\
\hline 18 & ${ }^{157} \mathrm{Gd}$ & 1977 & Mughabghab (1973) \\
\hline 19 & ${ }^{141} \operatorname{Pr}$ & 1980 & Mughabghab (1973) \\
\hline
\end{tabular}

For Cooling Times Over 5 Years, Gd-155 Ranks 1.

$\cdot[$ De95] 


\section{EVALUATION METHODS}

\section{II.A. Thermal Cross Sections}

In the present work, we followed closely the same evaluation procedure of the various thermal cross sections as that described in [Mu81].

The capture cross section, $\sigma_{\gamma}$, for a single resonance at energy $E_{o}$, represented by the BreitWigner formalism, is given by:

$$
\begin{gathered}
\sigma_{\gamma}(E)=\sigma_{0} \frac{\Gamma_{\gamma}}{\Gamma} \sqrt{\frac{E_{0}}{E}} \frac{1}{1+y^{2}}, \\
y=\frac{2}{\Gamma}\left(E-E_{0}\right), \\
\sigma_{0}=\frac{2.608 \times 10^{6}}{E_{0}}\left(\frac{A+1}{A}\right)^{2} \frac{g \Gamma_{n}}{\Gamma} .
\end{gathered}
$$

In this relation, $\Gamma_{\mathrm{n}}, \Gamma_{\gamma}$, and $\Gamma$ are the scattering width, the radiative width, and total width of the resonance, respectively; $\sigma_{0}$ is the peak total cross section; $A$ is the atomic mass number; $g$ is the statistical spin weight factor defined below.

If $\mathrm{N} \mathrm{s-wave} \mathrm{resonances} \mathrm{of} \mathrm{a} \mathrm{nucleus} \mathrm{are} \mathrm{located} \mathrm{away} \mathrm{from} \mathrm{the} \mathrm{thermal} \mathrm{energy,} \mathrm{then} \mathrm{their}$ contribution to the thermal capture cross section can be approximated by the following expression:

$$
\sigma_{\gamma}(0.0253 \mathrm{eV})=4.099 \times 10^{6}\left(\frac{\mathrm{A}+1}{\mathrm{~A}}\right)^{2} \sum_{\mathrm{j}=1}^{\mathrm{N}} \frac{\mathrm{g} \Gamma_{\mathrm{n}}^{0} \Gamma_{\mathrm{i}}}{\mathrm{E}_{\mathrm{oj}}^{2}} .
$$

The spin-dependent scattering lengths, $a+$ and a, associated with spin states $I+1 / 2$ and $I-1 / 2$, where $I$ is the spin of the target nucleus, can be written as:

$$
\mathrm{a}_{ \pm}=\mathrm{R}^{\prime}+\sum_{\mathrm{j}=1}^{\mathrm{N}} \frac{\lambda_{\mathrm{j}} \Gamma_{\mathrm{nj}}}{2\left(\mathrm{E}-\mathrm{E}_{\mathrm{j}}\right)+i \Gamma_{\mathrm{j}}}
$$

where $\mathbf{R}^{\prime}$ is the potential scattering length and $\lambda$ is De Broglie's wave length divided by $2 \pi$. The summation is carried out over $\mathrm{N}$ s-wave resonances with the same spin. For those cases where resonances are located farther away from thermal energy region, the above relation can be simplified to the following:

$$
a_{ \pm}(f m)=R^{\prime}-2.277 \times 10^{3}\left(\frac{A}{A+1}\right)^{2} \sum_{j=1}^{N} \frac{\Gamma_{n i}^{0}}{E_{0 j}} .
$$


The total coherent scattering length for non-zero spin target nuclei, is then the sum of the spin-dependent coherent scattering widths, $a_{+}$and $a_{\text {, }}$ weighted by the spin statistical factors, $g_{+}$and g.:

$$
\begin{gathered}
a=g_{+} a_{+}+g_{-} a_{-} \\
g_{+}=(I+1) /(2 I+1) \\
g_{.}=I /(2 I+1)
\end{gathered}
$$

The coherent, incoherent, and total scattering cross sections can then be expressed in terms of the scattering lengths by the following relations:

$$
\begin{aligned}
\sigma_{\text {coh }} & =4 \Pi\left(g_{+} a_{+}+g_{-} a_{-}\right)^{2} \\
\sigma_{\text {irc }}(\operatorname{spin}) & =4 \pi g_{+} g_{-}\left(a_{+}-a_{-}\right)^{2} \\
\sigma_{s} & =4 \pi\left(g_{+} a_{+}^{2}+g_{-} a_{-}^{2}\right)
\end{aligned}
$$

To calculate capture cross sections, coherent scattering amplitudes, and various spindependent scattering cross sections in terms of neutron-resonance parameters, the above relations, Eq. 1 to Eq. 8, were applied. These relations were previously implemented in the BNL computer code PSY325, which was used extensively in previous studies [Mu81, Mu84], as well as in the present evaluation.

If the results of the cross-section calculations do not agree with measurements within the uncertainty limits, then one or two negative energy (bound) levels are invoked. As a first step in the determination of the parameters of a bound level, we called upon Eq. 2 and Eq. 4. In addition, we assumed the radiative width of the bound level to be equal to the average s-wave radiative width of the nucleus under study. This quantity, as well as the potential scattering radius, is obtained from either measurements or the systematic investigations of average neutron resonance parameters, as well as optical model computations [Mu81, Mu84].

For target nuclei with non-zero spin, we relied in some favorable cases on gamma-ray spectra measurements of thermal neutrons to deduce the spins of the bound levels. In other cases, where two bound levels were stipulated, we made assumptions on their individual contributions. To carry out the final adjustments of the parameters of the bound level, the INTER computer code was used. In the final step of the analysis, the point wise energy-dependent cross sections were generated by the ENDF programs and then were compared graphically with measurements. Furthermore, the Westcott $\mathrm{g}$ factors, the capture resonance integrals, and the Maxwellian capture cross sections at a temperature of $30 \mathrm{keV}$ were calculated by the INTER computer code. 


\section{II.B. Resolved Resonance Region}

\section{Assignment of Orbital Angular Momentum Quantum Numbers}

The Bayesian approach [Mu81, Sm91] is adopted in this evaluation to distinguish p-wave from s-wave resonances for cases where the orbital angular momentum, $l$, have not been determined from measurements. Even though there is a potential danger of incorrect assignments of $l$ [Mu81, Ga81], the Bayesian approach was used in several cases in the past [for instance, Bo68, Mi79].

For a resonance with a neutron width weighted by the spin statistical factor, $g \Gamma_{n}$, the probability that this resonance is a p-wave resonance is given according to Bayes' theorem of conditional probability by:

$$
P\left(p \mid g \Gamma_{n}\right)=\frac{P\left(g \Gamma_{n} \mid p\right) P_{p}}{P\left(g \Gamma_{n} \mid s\right) P_{s}+P\left(g \Gamma_{n} \mid p\right) P_{p}+P\left(g \Gamma_{n} \mid d\right) P_{d}+\cdots},
$$

where $P\left(g \Gamma_{n} \mid s\right)$ is the probability that the neutron width is $g \Gamma_{n}$ if the resonance is an s-wave resonance, and $P_{s}, P_{p}$, and $P_{d}$ are a priori probabilities that the resonance is an $\mathrm{s}-, \mathrm{p}-$, or $\mathrm{d}$-wave resonance, respectively. Restricting the analysis to $s-$ and $p$-wave resonances, the above equation reduces to:

$$
P\left(p \mid g \Gamma_{n}\right)=\left(1+\frac{P\left(g \Gamma_{n} \mid s\right)\left\langle D_{1}\right\rangle}{P\left(g \Gamma_{n} \mid p\right)\left\langle D_{0}\right\rangle}\right)^{-1}
$$

where the level-spacing ratio $\left\langle D_{1}\right\rangle /\left\langle D_{0}\right\rangle$ is substituted for the ratio $P_{s} / P_{p}$. The probability density functions (pdf) for $\mathrm{s}-$ and $\mathrm{p}$-wave resonances were derived [Oh99] from the $\chi^{2}$ distribution proposed by Porter and Thomas[Po56]. The results are:

for s-wave,

$$
p\left(x_{0}\right) d x_{0}=\left(w_{01} \sqrt{\frac{w_{01}}{g_{01}} \frac{S_{0}}{S_{01}}} \frac{\exp \left(-\frac{w_{01} x_{0}}{2 g_{01}} \frac{S_{0}}{S_{01}}\right)}{\sqrt{2 \pi x_{0}}}+w_{02} \sqrt{\frac{w_{02}}{g_{02}} \frac{S_{0}}{S_{02}}} \frac{\exp \left(-\frac{w_{02} x_{0}}{2 g_{02}} \frac{S_{0}}{S_{02}}\right)}{\sqrt{2 \pi x_{0}}}\right) d x_{0},
$$

for p-wave,

$$
p\left(x_{1}\right) d x_{1}=\left(w_{11} \sqrt{\frac{3 w_{11}}{g_{11}} \frac{S_{1}}{S_{11}}} \frac{\exp \left(-\frac{3 w_{11} x_{1}}{2 g_{11}} \frac{S_{1}}{S_{11}}\right)}{\sqrt{2 \pi x_{1}}}+\frac{3 w_{12}^{2}}{g_{12}} \frac{S_{1}}{S_{12}} \exp \left(-\frac{3 w_{12} x_{1}}{g_{12}} \frac{S_{1}}{S_{12}}\right)\right.
$$




$$
\left.+\frac{3 w_{13}^{2}}{g_{13}} \frac{S_{1}}{S_{13}} \exp \left(-\frac{3 w_{13} x_{1}}{g_{13}} \frac{S_{1}}{S_{13}}\right)+w_{14} \sqrt{\frac{3 w_{14}}{g_{14}} \frac{S_{1}}{S_{14}}} \frac{\exp \left(-\frac{3 w_{14} x_{1}}{2 g_{14}} \frac{S_{1}}{S_{14}}\right)}{\sqrt{2 \pi x_{1}}}\right) d x_{1},
$$

In these relations, $x_{0}=g \Gamma_{n}^{0} /\left\langle g \Gamma_{n}^{0}\right\rangle$ and $x_{1}=g \Gamma_{n}^{1} /\left\langle g \Gamma_{n}^{1}\right\rangle . w_{l j}$ and $g_{l j}$ are the weights and the statistical factors, respectively, corresponding to the $j$-th resonance spin, $J_{j}$, arranged in ascending order, which can be formed from a given $l$ value and target spin, I. The weight is defined by the ratio of the number of $J_{j} l$-wave resonances to the total number of $l$-wave resonances such that $w_{l j}=\left\langle D_{l}\right\rangle /\left\langle D_{l j}\right\rangle$. Note that for spin zero target nuclides, weights $w_{01}$ and $w_{02}$ are 1 and 0 , respectively. Concerning the pdf for a p-wave resonance, the second and third terms in Eq. 12 take into account the degree of freedom of 2 for the same $J$ formation. Thus, in Eq. 12A, weights are set as $w_{12}=w_{13}=0$ for $I=0$, $w_{13}=0$ for $I=1 / 2$, and $w_{11}=0$ for $I=1$. Finally, in the derivation of Eq. 11 and Eq. 12, the neutron strength function is defined by:

$$
S_{l}=\left\langle g \Gamma_{n}^{l}\right\rangle /(2 l+1)\left\langle D_{l}\right\rangle \text { and } S_{l j}=\left\langle\Gamma_{n j}^{l}\right\rangle /\left\langle D_{l j}\right\rangle
$$

The J-dependent average level spacing is calculated by the Bethe formula:

$$
\frac{1}{\left\langle D_{l j}\right\rangle}=C\left(2 J_{j}+1\right) \exp \left(-\frac{\left(J_{j}+1 / 2\right)^{2}}{2 \sigma^{2}}\right),
$$

where $\sigma$ is the spin dispersion parameter. The constant $C$ is obtained from $\left\langle D_{0}\right\rangle$ and the relation $1 /\left\langle D_{0}\right\rangle=\sum_{j} 1 /\left\langle D_{0_{j}}\right\rangle$.

By applying Eq. $11 \mathrm{~A}$ and Eq. 12A to Eq. 10, along with additional relations, such that:

$$
d x_{0}=\frac{d\left(g \Gamma_{n}\right)}{S_{0}\left\langle D_{0}\right\rangle \sqrt{E} V_{0}} \text { and } d x_{1}=\frac{d\left(g \Gamma_{n}\right)}{3 S_{1}\left\langle D_{1}\right\rangle \sqrt{E} V_{1}}
$$

where the penetrabilities, $V_{0}=1$ and $V_{1}=k^{2} R^{2} /\left(1+k^{2} R^{2}\right)$, we can calculate the probability that a resonance at energy $E \mathrm{eV}$ and a width $=g \Gamma_{\mathrm{n}}$ is a p-wave resonance. However, such a calculation requires detailed information, such as $S_{l j}$, which may not be available. Therefore, some simplification is necessary.

When we assume the $(2 J+1)$ law of level density, the weight becomes $w_{l j}=g_{l j} / G_{l}$, where $G_{l} \equiv \sum_{j} g_{l j}$. Note that $G_{0}=1$ whereas $G_{1}=3$ for $\mathrm{I}=0, \mathrm{G}_{1}=9 / 4$ for $\mathrm{I}=1 / 2$, and $\mathrm{G}_{1}=2$ for $\mathrm{I} \geq 1$. In addition, with the relation $S_{l j}=\mu_{l j} S_{l}$, where $\mu_{l j}$ is the multiplicity of $J_{j}$, Eq. $11 \mathrm{~A}$ and Eq. $12 \mathrm{~A}$ become: 


$$
\begin{aligned}
p\left(x_{0}\right) d x_{0} & =\frac{\exp \left(-\frac{x_{0}}{2}\right)}{\sqrt{2 \pi x_{0}}} d x_{0}=\frac{\exp \left(-\frac{g \Gamma_{n}}{2\left\langle D_{0}\right\rangle S_{0} V_{0} \sqrt{E}}\right)}{\sqrt{2 \pi g \Gamma_{n} \sqrt{E}\left\langle D_{0}\right\rangle S_{0} V_{0}}} d\left(g \Gamma_{n}\right), \text { and } \\
p\left(x_{1}\right) d x_{1} & =\left\{\alpha \sqrt{\frac{3}{G_{1}}} \frac{1}{\sqrt{2 \pi x_{1}}}+(1-\alpha) \frac{3}{2 G_{1}}\right\} \exp \left(-\frac{3 x_{1}}{2 G_{1}}\right) d x_{1} \\
& =\left(\frac{\alpha}{\sqrt{2 \pi g \Gamma_{n} \sqrt{E} V_{1}\left\langle D_{0}\right\rangle S_{1}}}+\frac{1-\alpha}{2 \sqrt{E} V_{1}\left\langle D_{0}\right\rangle S_{1}}\right) \exp \left(-\frac{g \Gamma_{n}}{2 \sqrt{E} V_{1}\left\langle D_{0}\right\rangle S_{1}}\right) d\left(g \Gamma_{n}\right),
\end{aligned}
$$

where $\alpha=1$ for $\mathrm{I}=0, \alpha=2 / 3$ for $\mathrm{I}=1 / 2$, and $\alpha=1 / 2$ for $\mathrm{I} \geq 1$. Applying Eq. $11 \mathrm{~B}$ and Eq. $12 \mathrm{~B}$ to Eq. (10) results in:

$$
P\left(p \mid g \Gamma_{n}\right)=\left[1+\frac{\left\langle D_{1}\right\rangle}{\left\langle D_{0}\right\rangle} \sqrt{\frac{S_{1} V_{1}}{S_{0} V_{0}}} \frac{\exp \left\{\frac{g \Gamma_{n}}{2\left\langle D_{0}\right) \sqrt{E}}\left(\frac{1}{S_{1} V_{1}}-\frac{1}{S_{0} V_{0}}\right)\right\}}{\alpha+(1-\alpha) \sqrt{\frac{\pi g \Gamma_{n}}{2 \sqrt{E}\left\langle D_{0}\right\rangle S_{1} V_{1}}}}\right]^{-1} .
$$

This formula is applied in the computer code, PTANAL (see Appendix B). Note that $\left\langle D_{1}\right\rangle /\left\langle D_{0}\right\rangle$, as well as $\alpha$, depends on the target spin. In general, since the known values of $S_{1}$ are less certain than those of $S_{0}$, a set of probabilities was calculated for three different values of $S_{1}$ to access the sensitivity of the calculated probabilities on the p-wave strength function. We note that, regardless of the magnitude of the calculated probability, the $l$ value of a resonance, as determined from measurements, was not altered.

\section{Assignment of Resonance Spins}

Several methods to assign the spin $J$ of a resonance from measurements are available. For details, refer to \{Mu81]. However, for weak and high-energy resonances, spins are difficult to measure. For those resonances, a method of random assignment is applied in the present study. The probability that the spin is $J_{j}$ is calculated by:

$$
P\left(J_{j}\right)=\frac{N_{l j}}{\sum_{i} N_{l i}}=\frac{1 /\left\langle D_{l j}\right\rangle}{\sum_{i} 1 /\left\langle D_{l i}\right\rangle} .
$$


When we assume $(2 J+1)$ law, Eq. 17 becomes:

$$
P\left(J_{j}\right)=\frac{\left(2 J_{j}+1\right)}{2(2 I+1)} \frac{1}{G_{l}} .
$$

With the above equation as the pdf, and on the basis of a random number, uniform in the $[0,1]$ interval, the $J$ value of a resonance is assigned. 


\section{II.C. Unresolved Resonance Region}

1. Determination of Average Level Spacings and Strength Functions

After the determination of $l$ values for all resonances, the reduced neutron widths are analyzed in terms of the Porter-Thomas distribution [Po 57] to obtain the average reduced neutron width, the average level spacing and the neutron strength function for the unresolved resonance region. If the number of measured resonances is large enough for a statistical sample, the distribution of reduced neutron widths is analyzed in terms of the Porter-Thomas (PT) distribution.

$$
p(x) d x=\frac{e^{-x / 2}}{\sqrt{2 \pi x}} d x, x=\frac{g \Gamma_{n}^{l}}{\left\langle g \Gamma_{n}^{l}\right\rangle} .
$$

Its corresponding cumulative distribution function (cdf) is given by:

$$
P(x) \equiv \int_{0}^{x} p(x) d x=\operatorname{erf}(\sqrt{x / 2}) .
$$

This pdf is exactly the same as Eq. 11B for s-wave resonances but slightly differs from Eq. 12B for $\mathrm{p}$-wave resonances. Figure 1 shows the complement distribution function, cdf, i.e. $1-P(x)$, corresponding to Eq. 12B for the p-wave case. Note that Eq. 12B is reduced to Eq. 19 for $\mathrm{I}=0$ nuclide. Maximum relative difference of about $10 \%$ between $I=0$ and $I \geq 1$ cases is observed. Note that the cdf for p-wave case corresponding to $\mathrm{Eq} .12 \mathrm{~B}$ is:

$$
P(x)=\alpha \operatorname{erf}\left(\sqrt{\frac{3 x}{2 G_{1}}}\right)+(1-\alpha)\left\{1-\exp \left(-\frac{3 x}{2 G_{1}}\right)\right\} .
$$

For computational brevity, Eq. 20 was adopted in the analysis for both s- and p-wave resonances. As will be described later, because of the small number of assigned $\mathrm{p}$-wave resonances, the average parameters obtained from such analysis have to be adjusted to reproduce the capture cross sections in the unresolved resonance region in most cases. Thus an approximate model is satisfactory for the fitting of p-wave reduced widths. However, derived rigorous cdf s (Eq. 21) will be implemented in a later version of the PTANAL program.

After sorting $M$ measured $l$-wave reduced neutron widths in descending order, their distribution is fitted to the complement distribution function, cdf, using the following model:

$$
\begin{aligned}
& 1+\varepsilon_{1}=N_{r}\left\{1-\operatorname{erf}\left(\sqrt{g \Gamma_{n 1}^{l} / 2\left\langle g \Gamma_{n}^{l}\right\rangle}\right)\right\}, \\
& 2+\varepsilon_{2}=N_{r}\left\{1-\operatorname{erf}\left(\sqrt{g \Gamma_{n 2}^{l} / 2\left\langle g \Gamma_{n}^{l}\right\rangle}\right)\right\},
\end{aligned}
$$




$$
m+\varepsilon_{m}=N_{r}\left\{1-e r f\left(\sqrt{g \Gamma_{n m}^{l} / 2\left(g \Gamma_{n}^{l}\right\rangle}\right)\right\}
$$

where $N_{r}$ is the number of 'expected' total number of $l$-wave resonances and $\left\langle g \Gamma_{n}^{l}\right\rangle$ is the average reduced neutron width. Since resonances with small neutron widths are usually missed in measurements, it is necessary to exclude resonances whose reduced widths are smaller than a certain magnitude. Then the number of resonances is reduced to $m$ from a total $M$ measured resonances. By setting a cutoff value, i.e. minimum magnitude of reduced width, the effect of missed small resonances on the resulting average parameters is reduced significantly. The error term $\varepsilon_{i}$ is the propagated error from $g \Gamma_{n i}^{l} \pm \delta g \Gamma_{n i}^{l}$, which is approximately calculated by:

$$
\varepsilon_{i}=M \frac{\exp \left(-\frac{g \Gamma_{n i}^{l}}{2 \overline{g \Gamma_{n}^{l}}}\right)}{\sqrt{2 \pi g \Gamma_{n i}^{l} \overline{g \Gamma_{n}^{l}}}} \delta g \Gamma_{n i}^{l}, \overline{g \Gamma_{n}^{l}}=\sum g \Gamma_{n i}^{l} / M
$$

Now two parameters, $N_{r}$ and $\left\langle g \Gamma_{n}^{l}\right\rangle$, are determined through the fitting procedure. The Levenberg-Marquardt method $[\operatorname{Pr} 86]$ is used in this analysis. For $\left\langle D_{l}\right\rangle$ and $\left\langle g \Gamma_{n}^{l}\right\rangle$, the uncertainty of each quantity consists of two parts:

$$
\frac{\delta\left\langle D_{l}\right\rangle}{\left\langle D_{l}\right\rangle}=\sqrt{\frac{\operatorname{Var}\left(N_{r}\right)}{N_{r}^{2}}+\frac{0.52^{2}}{N_{r}}} \text { and } \frac{\delta\left\langle g \Gamma_{n}^{l}\right\rangle}{\left\langle g \Gamma_{n}^{l}\right\rangle}=\sqrt{\frac{\operatorname{Var}\left(\left\langle g \Gamma_{n}^{l}\right\rangle\right)}{\left\langle g \Gamma_{n}^{l}\right\rangle^{2}}+\frac{2}{N_{r}}}
$$

The first term originates from the fitting, and the second term is the inherent uncertainty. The variances of $N_{r}$ and $\left\langle g \Gamma_{n}^{l}\right\rangle$, as well as the covariance of these two parameters, are obtained from the fitting analysis. The inherent uncertainty of $\left\langle D_{l}\right\rangle$ is adopted from Wigner's surmise [Wi57] and that of $\left\langle g \Gamma_{n}^{l}\right\rangle$ from the Porter and Thomas distribution [Po56]. Note that the uncertainty of the level spacing, obtained by Dyson and Mehta [Dy63], $\delta D_{l} / D_{l}=0.45 / N \cdot \sqrt{\ln N+2.18}$, is much smaller than that obtained from Wigner's surmise. The resulting neutron strength function with a known energy interval $\Delta E$ becomes:

$$
S_{l}=\frac{\left\langle g \Gamma_{n}^{l}\right\rangle N_{r}}{(2 l+1) \Delta E}
$$




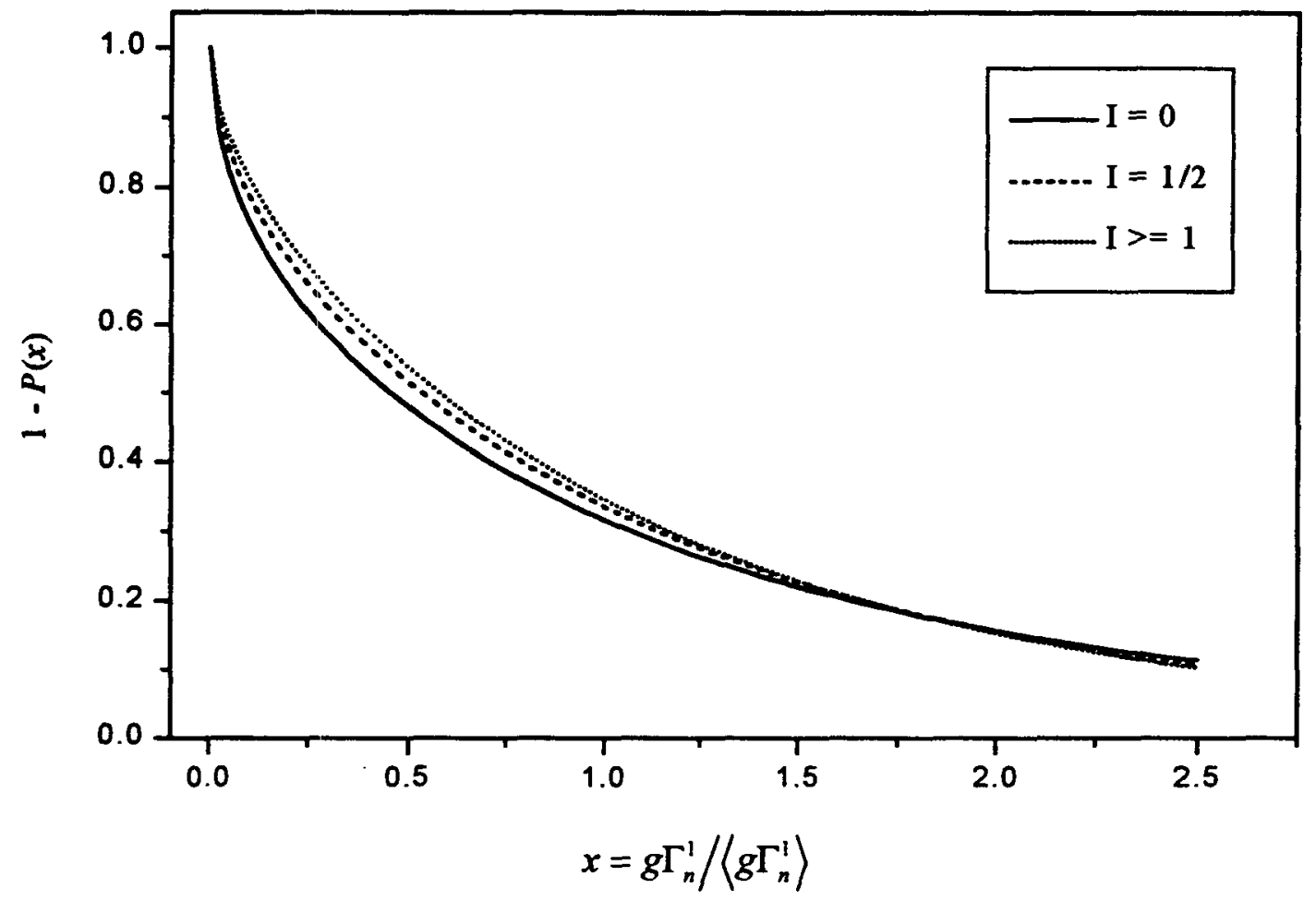

Fig. 1. Complement cdf for $p$-wave Neutron Reduced Widths

The corresponding relative uncertainty of $S_{l}$ is:

$$
\frac{\delta S_{l}}{S_{l}}=\sqrt{\left(\frac{\operatorname{Var}\left(N_{r}\right)}{N_{r}^{2}}+\frac{2 \operatorname{Cov}\left(N_{r},\left\langle g \Gamma_{n}^{l}\right\rangle\right)}{N_{r}\left\langle g \Gamma_{n}^{l}\right\rangle}+\frac{\operatorname{Var}\left(\left\langle g \Gamma_{n}^{l}\right\rangle\right)}{\left\langle g \Gamma_{n}^{l}\right\rangle^{2}}\right)+\frac{0.52^{2}+2}{N_{r}}}
$$

In most cases, analyzed in this work, the uncertainty due to the fitting is much smaller than the inherent uncertainty. 


\section{Determination of Average Radiative Widths}

Average radiative widths of neutron resonances are determined from measurements in the resolved energy region by calculating the weighted, as well as unweighted, values. For nuclei with unmeasured radiative widths, we called upon the systematic study of s-, p- and d-wave radiative widths as a function of atomic mass number, as described in [Mu84]. In some cases, final adjustments of the radiative widths were made in order to fit measured capture cross sections in the unresolved energy region. In other cases, we were guided by the recent theoretical calculations of radiative widths on the basis of giant dipole resonance [Mu99]. 


\section{RESULTS AND DISCUSSION}

\section{III.A. ${ }^{95} \mathrm{Mo}$}

\section{Thermal Region}

Positive-energy resolved resonance parameters and an effective scattering radius, $\mathbf{R}^{\prime}=7.0 \mathrm{fm}$, were adopted from Mughabghab's BNL compilation [Mu81]. However, in the resent evaluation, the parameters of a bound level were determined to reproduce a thermal capture cross section of $13.6 \mathrm{~b}$ and a bound coherent scattering length of $6.93 \pm 0.06 \mathrm{fm}$ [Ko87a, K091]. A capture cross section of $13.6 \mathrm{~b}$ was obtained by weighting $14.0 \pm 0.5 \mathrm{~b}$ in the BNL compilation and $13.4 \pm 0.3 \mathrm{~b}$ of Koester et al. [Ko87a]. The derived bound level parameters are: $\mathrm{E}_{0}=-26.8 \mathrm{eV}, \Gamma_{\mathrm{n}}=126 \mathrm{meV}, \Gamma_{\gamma}=150 \mathrm{meV}$ and $\mathrm{J}=2$. As shown in Table 2, the capture cross sections from the various indicated sources are in good agreement with each other, within the associated uncertainty limits. However, the present thermal scattering cross section is larger than those reported in other evaluated libraries.

\section{Resolved Resonance Parameters}

Resonance parameters, extending to an energy of $2.14 \mathrm{keV}$, were adopted from the BNL compilation [Mu81] with minor revisions. For resonances at energies of $1.3407,1.4955,1.5895$, 1.7661 and $2.1301 \mathrm{keV}$ for which neutron scattering widths are not quoted in the BNL compilation, the $2 \mathrm{~g} \Gamma_{\mathrm{n}}$ values were deduced from the total cross section measurement of natural Mo [Wy68]. Because of the lack of data in the energy region 2.14-3.23 keV, resonances above $2.14 \mathrm{keV}$ were not included in the present evaluation. Of a total of 55 resonances, 20 (including 12 identified in the BNL compilation) were assigned as s-wave resonances, whereas 35 (7 identified in the BNL compilation) were assigned as p-wave on the basis of Bayesian analysis. Spins of resonances for which the spins were not determined were assigned randomly by assuming that the level density is proportional to $(2 \mathrm{~J}+1)$. It is worth noting that Bayesian analysis for the resonance at $630.0 \mathrm{eV}$ with a relatively large neutron width $(24 \mathrm{meV})$ suggests s-wave assignment. On the other hand, this resonance is determined as a p-wave resonance by measurements. Weighted averaging of the known twelve radiative widths, two of which are pwave resonances, resulted in an average value of $158 \pm 14 \mathrm{meV}$. However, because of the importance of valence capture of p-wave resonances in this mass region [Mu71], we adopted a radiative width of $180 \mathrm{meV}$ for $\mathrm{p}$-wave resonances [Mu76]. For s-wave resonances for which radiative widths are unknown, a value of $150 \mathrm{meV}$ was assumed.

The distribution of reduced neutron widths was analyzed by fitting the widths to a PorterThomas distribution. Figure 2 shows the fit for the cumulative number of s-wave resonances having a $\operatorname{Vg}_{\mathrm{g}}{ }_{\mathrm{n}}^{0}$ larger than the value indicated on the $\mathrm{x}$-axis. None of the weak resonances was excluded in the fitting procedure. The fit resulted in $\left\langle\mathrm{D}_{0}\right\rangle=80.7 \pm 13.1 \mathrm{eV}$ and $\left\langle\mathrm{g} \Gamma_{\mathrm{n}}{ }^{0}\right\rangle=3.64 \pm 1.25$ $\mathrm{meV}$. These values yield $s$-wave strength function of $S_{0}=0.45 \pm 0.16$. The present $\left\langle D_{0}\right\rangle$ is

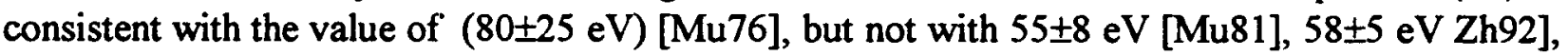
and $105 \pm 10 \mathrm{eV}$ [Re98]. The $S_{0}$ value is consistent with Musgrove's value $(0.48 \pm 0.10)$, slightly larger than that recommended in the BNL compilation $(0.35 \pm 0.07)$ [Mu81], and smaller than 
$(0.60 \pm 0.10)$ [Re98]. For $\mathrm{p}$-wave resonances, those with a $\mathrm{g} \Gamma_{\mathrm{n}}{ }^{1}$ value smaller than $25 \mathrm{meV}$ were excluded from the fitting procedure. The final fit, shown in Fig. 3, resulted in $\left\langle D_{1}\right\rangle=34.7 \pm 4.3$ $\mathrm{eV}$ and $\mathrm{S}_{1}=6.54 \pm 1.68$. The latter value is consistent with those reported in the BNL compilation

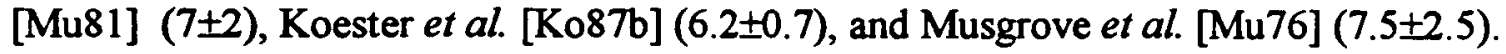

\section{Unresolved Resonance Parameters}

The present unresolved resonance region covers the energy range up to $206.27 \mathrm{keV}$, which corresponds to inelastic neutron scattering to the first excited level of ${ }^{95} \mathrm{Mo}$ at $204.12 \mathrm{keV}$. The average resonance parameters for $s-p-$, and d-wave resonances are provided. Since the upper energy range of the unresolved region is relatively high, $d$-wave contribution is not negligible. The level spacing varies with energy according to Gilbert-Cameron's level density formula with associated parameters adopted from Mughabghab and Dunford [Mu98a]. From the $(2 \mathrm{~J}+1)$ dependence of the level density, level spacings of $\mathrm{p}$ - and d-waves were assumed to be $1 / 2$ and $1 / 3$ of that for the s-wave resonances, respectively.

In the unresolved resonance region, average capture cross section measurements of Musgrove et al. [Mu76], which were corrected later by Allen et al. [Al82], and Kapchigashev and Popov [Ka64] were considered in this evaluation. These two sets show good agreement with each other. In the first attempt, we adopted values of $\left\langle D_{0}\right\rangle, S_{0}$ and $S_{1}$ deduced from the resolved resonances, $S_{2}$ read from a spherical optical model calculation in the BNL compilation [Mu84], and $\left\langle\Gamma_{\gamma}\right\rangle$ of $150 \mathrm{meV}$ for s- and d-wave whereas $180 \mathrm{meV}$ for $\mathrm{p}$-wave resonances. The resulting calculated capture cross section is larger by $10 \%$ to $15 \%$ than the measurements throughout the whole energy region. Subsequently, $\left\langle\mathrm{D}_{0}\right\rangle$ was adjusted to $69.4 \mathrm{eV}$ to obtain good agreement with the measurements as shown in Fig. 4. Note that the present $\left\langle D_{0}\right\rangle$ is twice that of $\left\langle D_{1}\right\rangle$ obtained from the analysis of resolved p-wave resonances. The finalized parameters of the present study are listed in the last column of Table 3 . We note that unresolved resonance parameters were not provided in the ENDF/B-VI and JEF-2.2 evaluations.

Maxwellian-averaged capture cross section for a temperature of $30 \mathrm{keV}$ was computed as $336 \mathrm{mb}$. The integration was performed up to an energy of $206 \mathrm{keV}$. The present cross section is compared with 292 $\pm 12 \mathrm{mb}$ [Wi87] (see Beer's compilation [Be92]) and 380 $\pm 50 \mathrm{mb}$ [Mu81]. 
Table 2. Thermal Characteristics $\left({ }^{95} \mathrm{Mo}\right)$

\begin{tabular}{|c|c|c|c|c|c|c|c|}
\hline Quantity & Unit & $\begin{array}{c}\text { BNL } \\
{[\mathrm{Mu} 81]}\end{array}$ & $\begin{array}{l}\text { 98CRC } \\
\text { [Ho98] } \\
\end{array}$ & $\begin{array}{l}\text { ENDF/ } \\
\text { B-VI }\end{array}$ & JEF-2.2 & $\begin{array}{c}\text { JENDL- } \\
3.2 \\
\end{array}$ & Present \\
\hline $\mathbf{R}^{\prime}$ & $\mathrm{fm}$ & $7.0 \pm 0.2$ & & 5.50 & 7.0 & 7.0 & 7.0 \\
\hline$\sigma_{\gamma}^{0}$ & barn & $14.0 \pm 0.5$ & $13.4 \pm 0.3$ & 14.6 & 14.0 & 14.0 & 13.6 \\
\hline$\sigma_{\mathrm{s}}{ }^{0}$ & barn & & & 2.48 & 5.21 & 5.57 & 6.41 \\
\hline$g_{w}^{*}$ & & & & 0.9990 & 0.9987 & 0.9994 & 1.0000 \\
\hline $\begin{array}{c}\text { RI- } \\
\text { capt. }\end{array}$ & barn & $109 \pm 5$ & $109 \pm 5$ & 113 & 110 & 119 & 111 \\
\hline RI-total ${ }^{* *}$ & barn & & & 291 & 306 & 334 & 315 \\
\hline
\end{tabular}

*Westcott factor for capture cross section.

** Integrated from $0.5 \mathrm{eV}$ to $100 \mathrm{keV}$ with $1 / \mathrm{E}$ spectrum.

Table 3. Average Resonance Parameters for the Unresolved Resonance Region $\left({ }^{95} \mathrm{Mo}\right)$

\begin{tabular}{|c|c|c|c|c|c|c|c|}
\hline \multirow{2}{*}{ Quantity } & Unit & \multirow{2}{*}{$\begin{array}{c}\text { BNL } \\
{[\mathrm{Mu} 81]}\end{array}$} & $\begin{array}{c}\text { ENDF/ } \\
\text { B-VI }\end{array}$ & JEF-2.2 & JENDL- & \multicolumn{2}{|c|}{ Present } \\
\cline { 6 - 8 } & & & & $\begin{array}{c}\text { PT } \\
\text { Analysis }\end{array}$ & Adopted \\
\hline $\mathrm{R}^{\prime}$ & $\mathrm{Fm}$ & $7.0 \pm 0.2$ & & & 6.68 & & 7.0 \\
\hline$\left\langle\mathrm{D}_{0}\right\rangle$ & $\mathrm{eV}$ & $55 \pm 8$ & & & 78.86 & $80.7 \pm 13.1$ & $69.4^{* *}$ \\
$\mathrm{~S}_{0}$ & $\times 10^{-4}$ & $0.35 \pm 0.07$ & & & 0.37 & $0.45 \pm 0.16$ & 0.45 \\
$\left\langle\Gamma_{\gamma 0}\right\rangle$ & $\mathrm{meV}$ & $160 \pm 20$ & & & 232 & & 150 \\
\hline$\left\langle\mathrm{D}_{1}\right\rangle$ & $\mathrm{eV}$ & & & & 39.43 & $34.7 \pm 4.3$ & $34.7^{* *}$ \\
$\mathrm{~S}_{1}$ & $\times 10^{-4}$ & $7 \pm 2$ & & & 5.48 & $6.54 \pm 1.68$ & 6.54 \\
$\left\langle\Gamma_{\gamma 1}\right\rangle$ & $\mathrm{meV}$ & & & & 232 & & 180 \\
\hline$\left\langle\mathrm{D}_{2}\right\rangle$ & $\mathrm{eV}$ & & & & 26.29 & & $23.1^{* *}$ \\
$\mathrm{~S}_{2}$ & $\times 10^{-4}$ & & & & 0.365 & & 1.70 \\
$\left\langle\Gamma_{\gamma 2}\right\rangle$ & $\mathrm{meV}$ & & & & 232 & & 150 \\
\hline
\end{tabular}

* Average parameters at the low energy range of the unresolved region $(2.04 \mathrm{keV})$.

** Value at the neutron separation energy of ${ }^{96} \mathrm{Mo}$. 


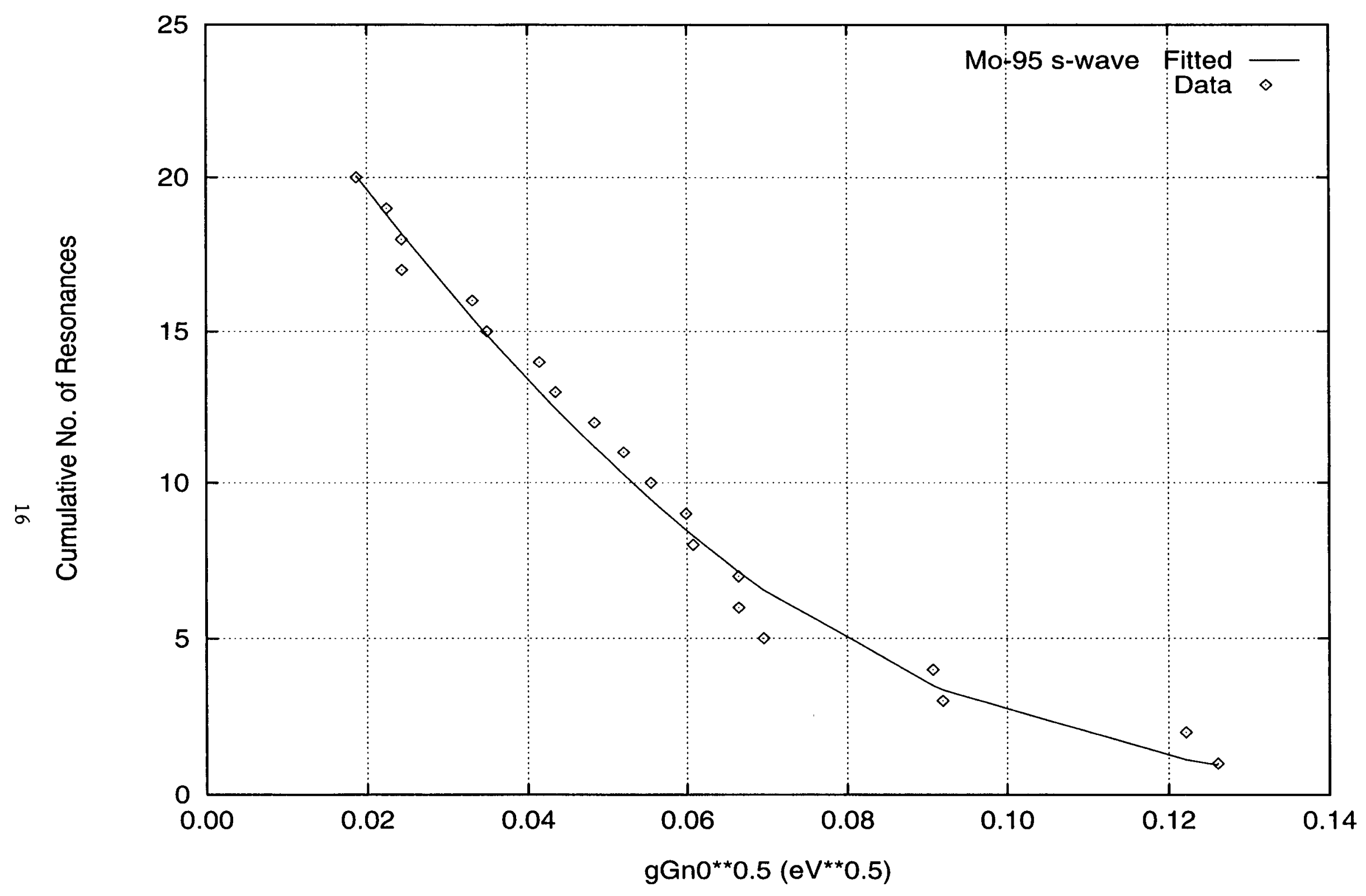

Fig. 2. Complement of the Cumulative Distribution of Neutron Reduced Widths ( ${ }^{95} \mathrm{Mo}$, s-wave) 


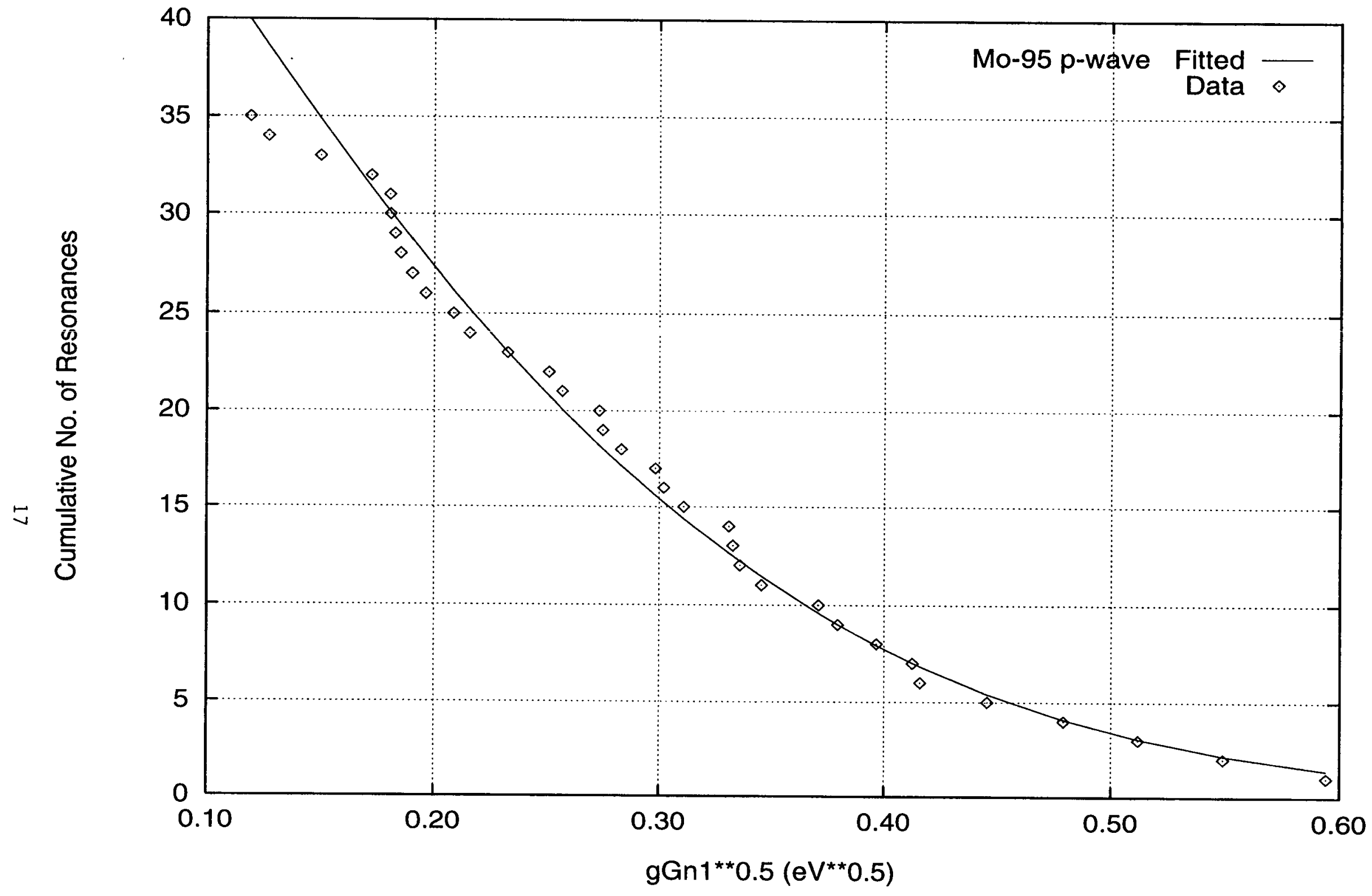

Fig. 3. Complement of the Cumulative Distribution of Neutron Reduced Widths $\left({ }^{95} \mathrm{Mo}\right.$, p-wave) 


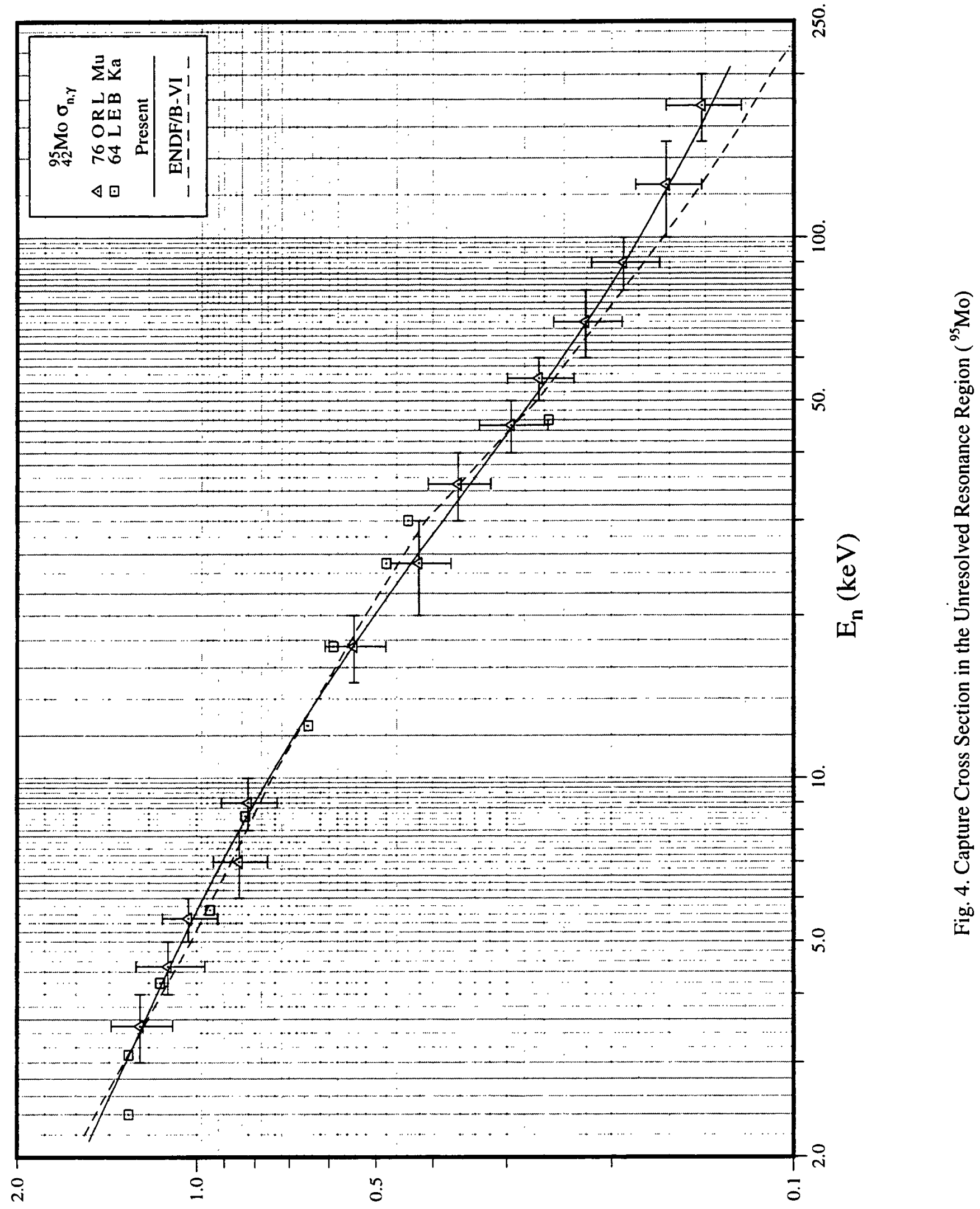

(q) 0 


\section{III.B. ${ }^{99} \mathrm{TC}$}

\section{Thermal Region}

The thermal capture cross section at $0.0253 \mathrm{eV}(2200 \mathrm{~m} / \mathrm{sec})$ and the bound coherent scattering amplitude were adopted from the BNL compilation [Mu81]. The capture cross section recently measured by Harada et al. [Ha95] was not considered since it was based on an incorrect half-life of ${ }^{100} \mathrm{Tc}$. Harada et al. used $15.5 \mathrm{sec}$, while the recent recommended value is $15.8 \mathrm{sec}$ [Au95]. A bound level was invoked to fit a coherent scattering amplitude of $b^{\prime}=6.8 \pm 0.3 \mathrm{fm}$ [Mu81] and the thermal capture cross section [Mu81]. To determine the parameters of the bound level, a potential scattering radius of $R^{\prime}=7.0 \mathrm{fm}$, based on systematics study and optical model calculations [Mu84], was applied in the analysis. The resulting parameters are: $E_{0}=-20.318$ $\mathrm{eV}, \mathrm{J}=4, \Gamma_{\mathrm{n}}=35.5 \mathrm{meV}$, and $\Gamma_{\gamma}=109.5 \mathrm{meV}$. Table 4 summarizes the present calculated thermal values which are compared with previous evaluations. The calculated Westcott factor for capture is $\mathrm{g}_{\mathrm{w}}=1.0036$.

\section{Resolved Resonance Parameters}

The BNL resonance parameter compilation of ${ }^{99} \mathrm{Tc}$ [Mu81] is based on available measurements prior to 1981. Recent measurements carried out at Geel by the Saclay group [Gu97, Ra97] reported resonance parameters up to $600 \mathrm{eV}$. This information was incorporated in the present evaluation. Because resonances above an energy of $1000 \mathrm{eV}$ are not resolved, the upper energy of the resolved resonance region was set at this energy. The favored spin of the first resonance at $5.61 \mathrm{eV}$ is 5 . Since spins of other resonances were not determined experimentally, these were assigned randomly on the basis of the $2 \mathrm{~J}+1$ law for the density of resonances. Figure 5 displays the result of the Porter-Thomas distribution analysis of reduced neutron widths, on the assumption that all resonances are treated as s-wave levels. Note that below $0.01(\mathrm{eV})^{1 / 2}$, there is an excess of weak resonances, indicating the presence of $p$-wave resonances. With this cutoff value, the analysis was carried out (Fig. 6), leading to an average swave level spacing, $D_{0}=15.4 \pm 2.0 \mathrm{eV}$, and an s-wave strength function, $S_{0}=0.43 \pm 0.10$. Angular momentum assignments $(l=0,1)$ were carried out with the help of Bayesian methodology. Figure 7 displays the Porter-Thomas distribution for $\mathrm{p}$-wave resonances. For a cutoff value of $\left(g \Gamma_{n}^{1}\right)^{1 / 2}>0.13(\mathrm{eV})^{1 / 2}$, we obtain the following values: $D_{1}=6.0 \pm 0.9 \mathrm{eV}$ and $S_{1}$ $=7.3 \pm 1.6$. The latter value is consistent with optical model calculation [Mu84]. A comparison between the present average resonance parameters and previous evaluations is shown in Table 5.

Special attention was paid to the evaluation of the radiative width of the first positiveenergy resonance at $5.58 \mathrm{eV}$. On the basis of recent [Gu97] and previous [Fi78, Wa70] measurements, we obtain $\Gamma_{Y}=140 \mathrm{meV}$ for this resonance.

\section{Unresolved Resonance Parameters}

We set the boundary between the resolved and unresolved energy regions at $991 \mathrm{eV}$. Since the first excited level of ${ }^{99} \mathrm{Tc}$ is $140.5108 \mathrm{keV}$, the upper limit of the unresolved resonance region was set to $141.94 \mathrm{keV}$. 
Three capture cross section measurements [Ch73, Li77, Ma82] are relevant to the unresolved energy region. When compared with the ORNL data [Ma82a], the RPI data [Li77] is high while the KFK data [Ch73] is low. As a starting point, the parameters obtained from the Porter-Thomas analysis in the resolved energy region were applied in the unresolved energy region. In addition, the average $p$-wave radiative width and the $d$-wave strength function are obtained from the systematics investigations [Mu84]. An energy-dependent level spacing with the relevant parameters of Mughabghab and Dunford [Mu98a], as well as a spin dispersion parameter of 2.8 [Mu98b], were applied in the calculation of the capture cross section. To fit the ORNL data [Ma82a], the average s-wave radiative width was adjusted from $173 \mathrm{meV}$ to 130 $\mathrm{meV}$. This value is consistent, within the error limits, with $115 \pm 17 \mathrm{meV}$ determined by Macklin [Ma82a]. In addition, an adjustment of the p-wave strength function was made from a value of 7.3 to 6.0 .

The final unresolved resonance parameters are listed in Table 6 and are compared with existing evaluated data files. The present parameters are also compared with those recommended in RIPL [Re98]: $\left\langle\mathrm{D}_{0}\right\rangle=12.8 \pm 1.8 \mathrm{eV}, \mathrm{S}_{0}=0.48 \pm 0.07$, and $\left\langle\Gamma_{\gamma 0}\right\rangle=160 \pm 50 \mathrm{meV}$. The capture cross section constructed with the present average parameters is shown in Fig. 8.

Maxwellian average capture cross section for a temperature of $30 \mathrm{keV}$ was calculated by the INTER computer code as $795 \mathrm{mb}$, which is consistent with a value of $782 \pm 40 \mathrm{mb}$ reported in Beer's compilation [Be92]. The calculation is performed up to $1 \mathrm{MeV}$ with the aid of supplementary capture cross section in the energy region from $141.94 \mathrm{keV}$ to $1 \mathrm{MeV}$, imported from ENDF/B-VI.

Table 4. Thermal Characteristics $\left({ }^{99} \mathrm{Tc}\right)$

\begin{tabular}{|c|c|c|c|c|c|c|c|}
\hline Quantity & Unit & $\begin{array}{c}\text { BNL } \\
{[\mathrm{Mu} 81]}\end{array}$ & $\begin{array}{c}98 \mathrm{CRC} \\
{[\mathrm{Ho98}]}\end{array}$ & $\begin{array}{c}\text { ENDF/B- } \\
\text { VI }\end{array}$ & JEF-2.2 & $\begin{array}{c}\text { JENDL- } \\
3.2\end{array}$ & Present \\
\hline$R^{\prime}$ & $\mathrm{fm}$ & $6.0 \pm 0.5$ & - & 7.91 & 6.0 & 6.0 & 7.0 \\
\hline$b^{\prime}$ & $\mathrm{fm}$ & $6.8 \pm 0.3$ & $6.8 \pm 0.3$ & 7.38 & & 5.22 & 6.75 \\
\hline$\sigma_{\gamma}$ & barn & $20 \pm 1$ & $23 \pm 2$ & 19.6 & 19.1 & 19.6 & 20.0 \\
\hline$\sigma_{s}$ & barn & - & - & 6.88 & 3.54 & 3.43 & 5.81 \\
\hline RI-capt. & barn & $340 \pm 20$ & $400 \pm 40$ & 350 & 304 & 312 & 312 \\
\hline
\end{tabular}

Table 5. Parameters of the $5.58 \mathrm{eV}$ Resonance of ${ }^{99} \mathrm{Tc}$

\begin{tabular}{|c|c|c|c|c|c|c|}
\hline Parameter & Unit & $\begin{array}{c}\text { BNL } \\
{[\text { Mu81] }}\end{array}$ & $\begin{array}{c}\text { ENDF/B- } \\
\text { VI* }\end{array}$ & JENDL-3.2 & $\begin{array}{c}\text { Gunsing } \\
{[\text { Gu97] }}\end{array}$ & Present \\
\hline$g \Gamma_{n}$ & $\mathrm{meV}$ & 1.755 & 2.25 & 1.885 & 1.864 & 1.806 \\
\hline$\Gamma_{r}$ & $\mathrm{meV}$ & $177 \pm 18$ & 134 & 168.1 & 149.2 & 140 \\
\hline
\end{tabular}

* ENDF/B-VI uses a bound resonance at $-6.4 \mathrm{eV}$. 
Table 6. Average Resonance Parameters for Unresolved Resonance Region $\left({ }^{99} \mathrm{Tc}\right)$

\begin{tabular}{|c|c|c|c|c|c|c|c|}
\hline \multirow{2}{*}{ Quantity } & \multirow{2}{*}{ Unit } & \multirow{2}{*}{$\begin{array}{c}\text { ENDF/B- } \\
\text { VI }\end{array}$} & JEF-2.2 & JENDL- & \multirow{2}{*}{$\begin{array}{c}\text { BNL } \\
\text { [Mu81] }\end{array}$} & $\begin{array}{c}\text { PT } \\
\text { Analysis }\end{array}$ & Adopted \\
\hline$R^{\prime}$ & $\mathrm{fm}$ & 7.91 & 6.80 & 6.216 & $6.0 \pm 0.5$ & & 7.00 \\
\hline$\left\langle D_{0}\right\rangle$ & $\mathrm{eV}$ & 12.1 & 18.34 & 20.6 & $10.7 \pm 1.8$ & $15.4 \pm 2.0$ & $15.4^{* *}$ \\
$S_{0}$ & $\times 10^{-4}$ & 0.43 & 0.55 & $0.54^{*}$ & $0.45 \pm 0.0$ & $5.43 \pm 0.10$ & 0.43 \\
$\left\langle\Gamma_{\gamma 0}\right\rangle$ & $\mathrm{meV}$ & 122 & 131 & 187 & 160 & & 130 \\
\hline$\left\langle D_{1}\right\rangle$ & $\mathrm{eV}$ & 6.10 & 9.49 & 10.29 & & $6.0 \pm 0.9$ & $7.68^{* *}$ \\
$S_{1}$ & $\times 10^{-4}$ & 3.88 & 8.94 & $5.52^{*}$ & & $7.3 \pm 1.6$ & 6.0 \\
$\left\langle\Gamma_{\gamma 1}\right\rangle$ & $\mathrm{meV}$ & 122 & 131 & 187 & & & 140 \\
\hline$\left\langle D_{2}\right\rangle$ & $\mathrm{eV}$ & - & 6.70 & 6.86 & & & $5.25^{* *}$ \\
$S_{2}$ & $\times 10^{-4}$ & - & 0.55 & $0.64^{*}$ & & & 1.0 \\
$\left\langle\Gamma_{\gamma 2}\right\rangle$ & $\mathrm{meV}$ & - & 131 & 187 & & & 130 \\
\hline
\end{tabular}

* Value at the low energy $(4.219 \mathrm{keV})$ range of the unresolved resonance region.

** Value at the neutron separation energy of ${ }^{100} \mathrm{Tc}$. 


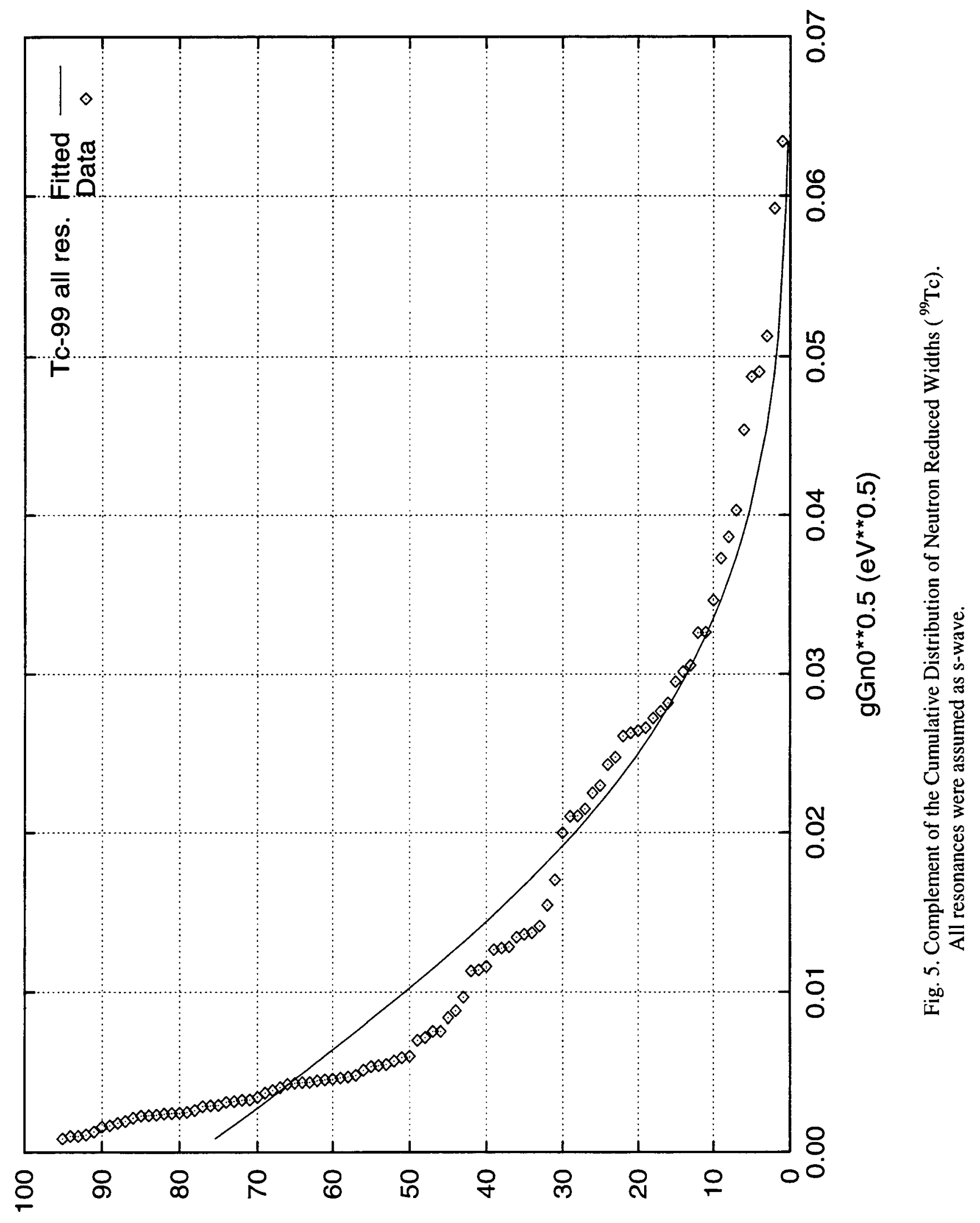

səoueuosey to 'ON әм!̣e|nunว 


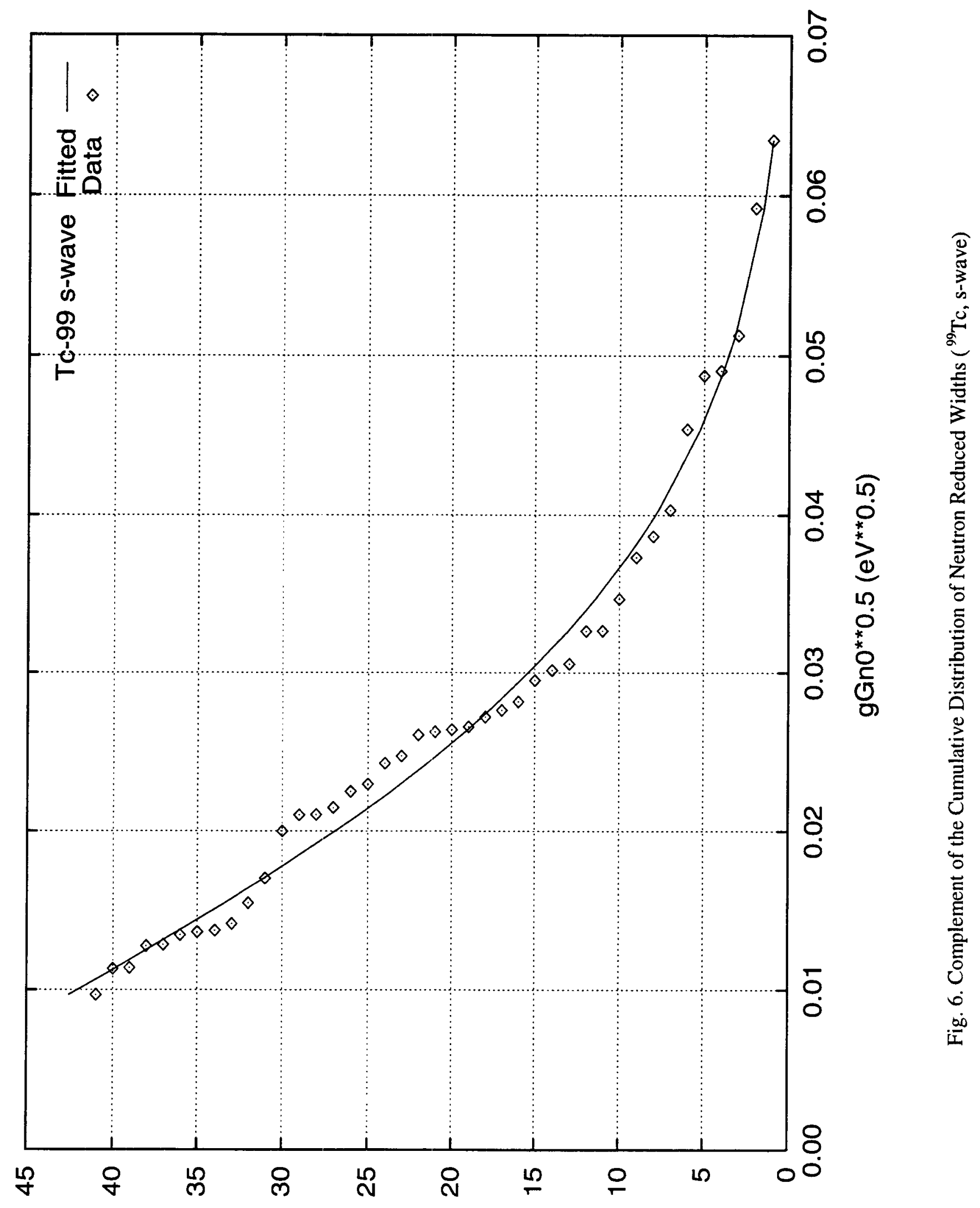




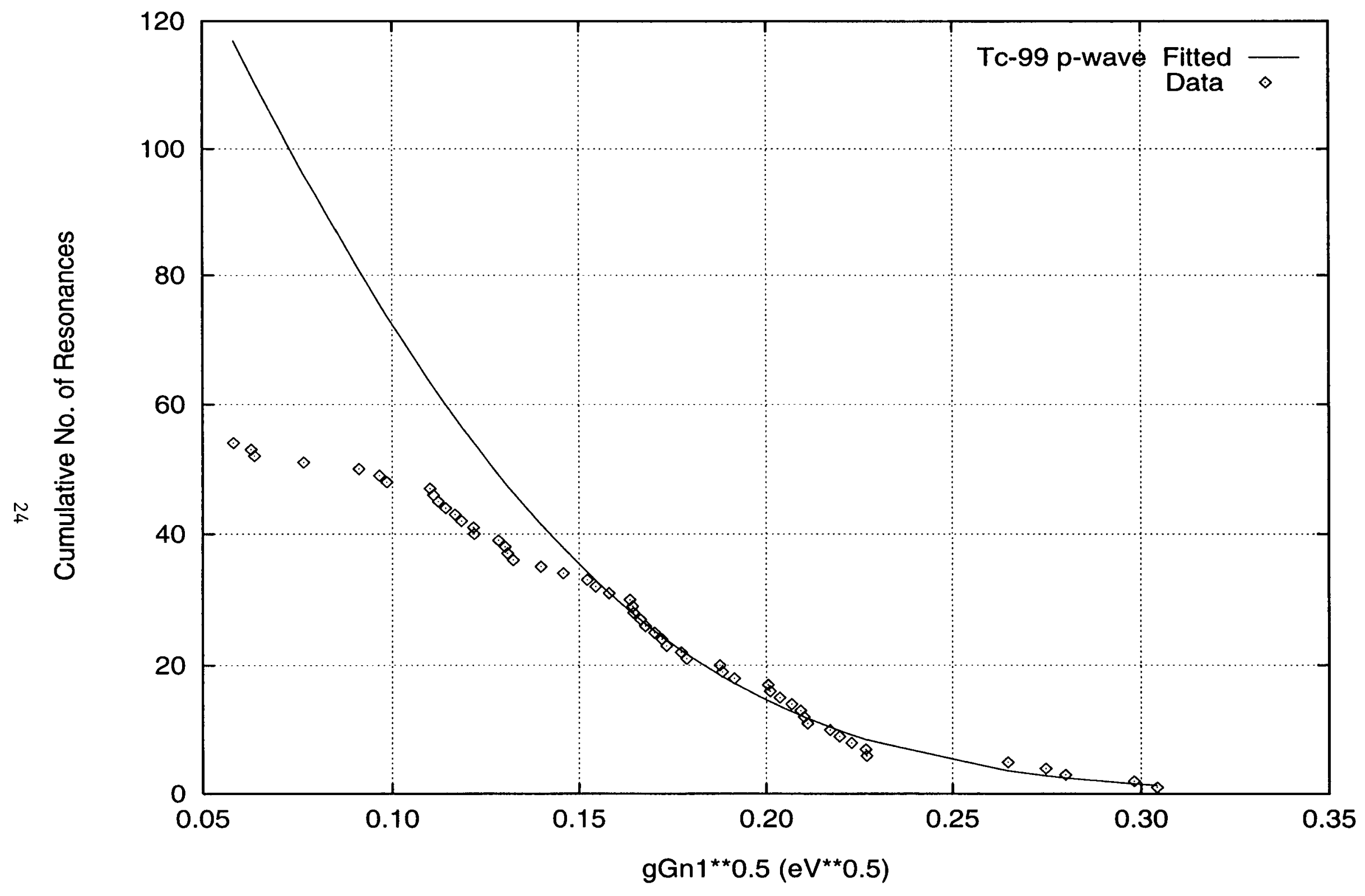

Fig. 7. Complement of the Cumulative Distribution of Neutron Reduced Widths $\left({ }^{99} \mathrm{Tc}\right.$, $\mathrm{p}$-wave) 


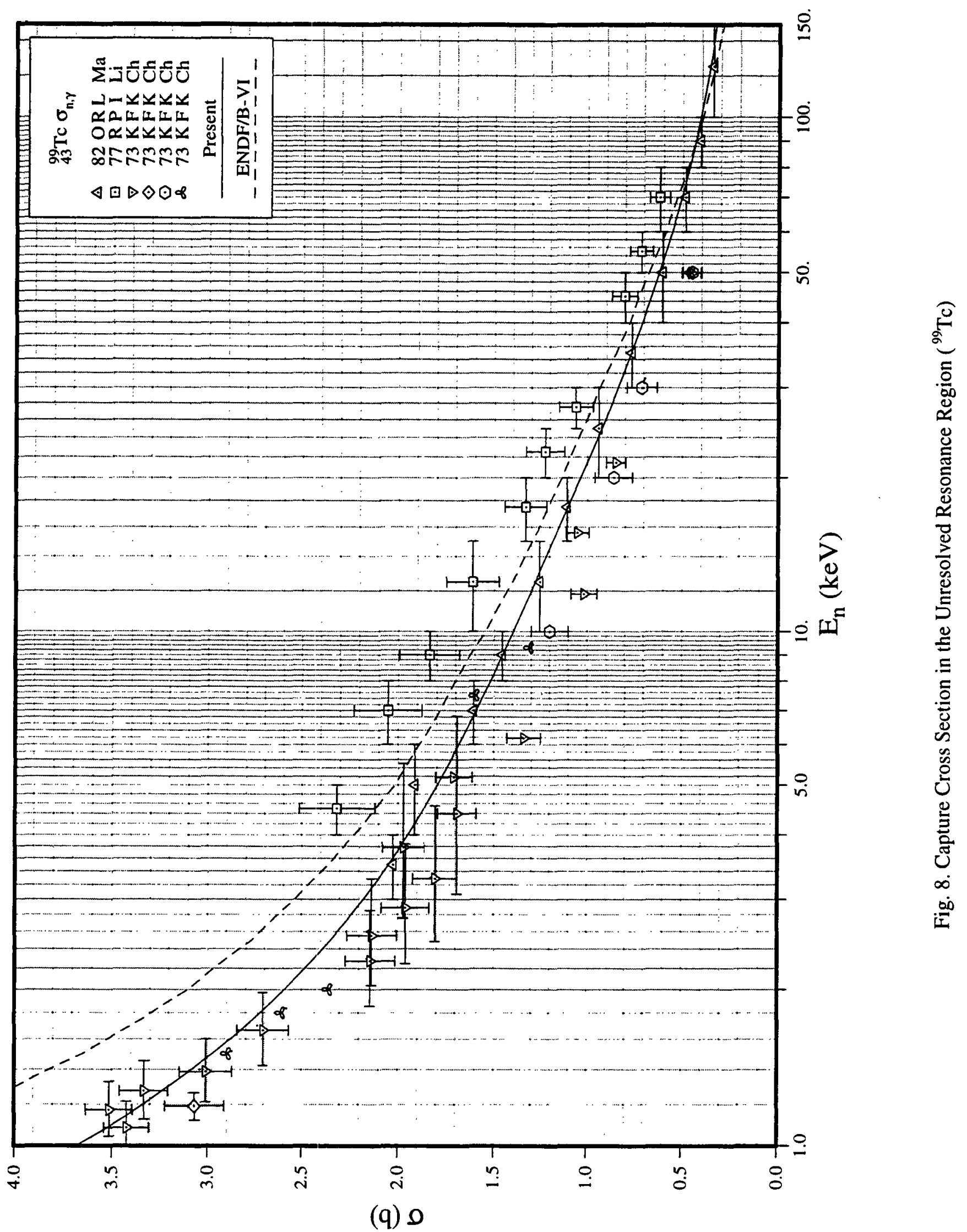


III.C. ${ }^{101} \mathbf{R u}$

\section{Thermal Region}

A capture cross section of $3.45 \mathrm{~b}$ is computed at $0.0253 \mathrm{eV}$ from the present evaluation of the resonance parameters. This value is consistent with Halperin's measurement [Ha65], which yields $\sigma_{\gamma}=3.0 \mathrm{~b}$, and with Mughabghab's BNL compilation [Mu81], $\sigma_{\gamma}=3.4 \pm 0.9 \mathrm{~b}$. The former value is obtained by correcting for reactor neutrons by using a resonance integral of $100 \mathrm{~b}$ as calculated from the resonance parameters. Bound levels are not required to describe the capture cross section at thermal energies. A comparison of our thermal cross sections, as well as resonance integrals, with other evaluations is made in Table 7.

\section{Resolved Resonance Parameters}

Measurement by Anufriev et al. [An85] in the energy region from thermal energy to 1720 $\mathrm{eV}$, which was not cited in the BNL compilation [Mu81], was included in the present evaluation. In addition, weak observed resonances, identified as belonging to ${ }^{101} \mathrm{Ru}$, were included in the present evaluation. Because of poor energy resolution [An85], the resonance parameters above $62 \mathrm{eV}$ were not considered. Above this energy, the resonance parameters of the BNL compilation were adopted. Because of lack of measurements in the energy region from 1.035 to $2.661 \mathrm{keV}$, Macklin and Halperin's [Ma80] resolved resonance parameters above $2.6 \mathrm{keV}$ were not included in this evaluation.

The average radiative width, calculated from the known 11 radiative widths below 1.035 $\mathrm{keV}$, is $184 \pm 10 \mathrm{meV}$; this value was assumed for resonances with unreported radiative widths. This average is consistent with the values, $180 \pm 15 \mathrm{meV}$ reported in the BNL compilation, $187 \pm 16 \mathrm{meV}$, determined by Macklin and Halperin [Ma80] for 10 resonances in the energy region $2.874-4.433 \mathrm{keV}$, and $175 \mathrm{meV}$ of Popov et al. [Po79]. However, it is smaller than $219 \pm 19 \mathrm{meV}$ determined by Anufriev et al. [An85]. Of a total of 48 resonances, 41 were assigned as s-wave and 7 as p-wave from the Bayesian analysis. Resonance spins were assigned randomly on the basis of the $(2 \mathrm{~J}+1)$ dependence of the level density to resonances for which the spin was not determined.

The distribution of reduced neutron widths was analyzed by fitting the widths to the Porter-Thomas distribution. As shown in Fig. 9 none of the weak resonances was excluded from the fitting. The fit resulted in $\left\langle\mathrm{D}_{0}\right\rangle=19.3 \pm 1.8 \mathrm{eV}$ and $\left\langle\mathrm{g} \Gamma_{\mathrm{n}}{ }^{0}\right\rangle=1.21 \pm 0.28 \mathrm{meV}$. These values yield s-wave strength function of $S_{0}=0.62 \pm 0.15$. The present $\left\langle D_{0}\right\rangle$ is consistent with values reported in the BNL compilation (D = 16 $\pm 2 \mathrm{eV}), \mathrm{RIPL}$ [Re98] $(18 \pm 3 \mathrm{eV})$, and Popov et al. [Po79] $(18 \pm 2 \mathrm{eV})$. The $S_{0}$ value is consistent with those of Macklin and Halperin [Ma80] $(0.59 \pm 0.04)$ and Popov et al. [Po79] $(0.61 \pm 0.13)$. However, it is somewhat larger than the values

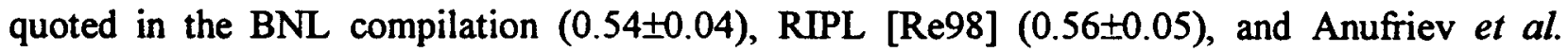
[An85] $(0.547 \pm 0.015)$. For $p$-wave resonances, a fit to the Porter-Thomas distribution yielded unreliable results due to the limited number of assigned $p$-wave resonances. 


\section{Unresolved Resonance Parameters}

The present unresolved resonance region covers the energy range from $1.035 \mathrm{keV}$ to $128.49 \mathrm{keV}$. The latter energy corresponds to the threshold energy of inelastic neutron scattering to the first excited level of ${ }^{101} \mathrm{Ru}$. In this region, the average resonance parameters for s-, p- and $\mathrm{d}$-wave were provided. Since the upper energy of the unresolved resonance region is relatively high, $d$-wave contribution is not negligible. An energy-dependent level spacing according to Gilbert-Cameron level density formula with associated parameters adopted from Mughabghab and Dunford [Mu98a] was applied in the calculations. On the basis of the $(2 \mathrm{~J}+1)$ dependence of the level density, level spacings for $p$ - and $d$-waves were assumed to be $1 / 2$ and $1 / 3$ of the s-wave spacing, respectively.

The average capture cross section measurement by Macklin and Halperin [Ma80], corrected later by Macklin and Winters [Ma81], was considered in this evaluation. As a first attempt in the evaluation of the capture cross section, the following parameters were implemented: 1) $\left\langle D_{0}\right\rangle$ and $S_{0}$ values which were derived from resolved resonances; 2) $S_{1}$ and $S_{2}$ values of Macklin and Haiperin; and 3$)\left\langle\Gamma_{\gamma}\right\rangle=184 \mathrm{meV}$ for s-, p- and d-wave resonances. These parameters resulted in a calculated capture cross section which is in good agreement with the measurement [Ma80, Ma81] below $100 \mathrm{keV}$, but above $100 \mathrm{keV} 5 \%$ it is larger than the measurement. Subsequently, to achieve an improved fit at the high energy, $S_{2}$ was reduced to 1.2. This $S_{2}$ value is consistent with reported measured values in the mass region around $A=100$ [Mu84, Fig. 5]. The finalized parameters of the present evaluation are listed in Table 8. The capture cross section data and the final calculated fit are shown in Fig. 10.

Maxwellian-averaged capture cross section for a temperature of $30 \mathrm{keV}$ was computed as $970 \mathrm{mb}$. In the INTER calculation up to $\mathrm{I} \mathrm{MeV}$, the capture cross sections for the energy region from $127 \mathrm{keV}$ to $1 \mathrm{MeV}$ were adopted from the ENDF/B-VI evaluation. The present cross section is compared with $996 \pm 40 \mathrm{mb}$ reported in Beer's compilation [Be92] and $1011 \mathrm{mb}$ in the BNL compilation [Mu81].

Table 7. Thermal Characteristics $\left({ }^{101} \mathrm{Ru}\right)$

\begin{tabular}{|c|c|c|c|c|c|c|c|}
\hline Quantity & Unit & $\begin{array}{c}\text { BNL } \\
\text { Compilation }\end{array}$ & $\begin{array}{c}\text { 98CRC } \\
\text { [Ho98] }\end{array}$ & $\begin{array}{c}\text { ENDF/ } \\
\text { B-VI }\end{array}$ & JEF-2.2 & $\begin{array}{c}\text { JENDL- } \\
3.2\end{array}$ & Present \\
\hline $\mathrm{R}^{\prime}$ & fm & & & 5.617 & 6.9 & 6.1 & 6.53 \\
\hline$\sigma_{\gamma}^{0}$ & barn & $3.4 \pm 0.9$ & $5 \pm 1$ & 3.43 & 3.41 & 3.36 & 3.45 \\
$\sigma_{\mathrm{s}}{ }^{0}$ & barn & & & 3.23 & 5.05 & 3.74 & 4.50 \\
\hline $\mathrm{gw}^{*}$ & & & & 1.003 & 1.001 & 1.001 & 1.0016 \\
\hline $\begin{array}{c}\text { RI- } \\
\text { capt. }\end{array}$ & barn & $100 \pm 20$ & $110 \pm 30$ & 111 & 111 & 100 & 111 \\
RI-total $^{* *}$ & barn & & & 184 & 200 & 173 & 200 \\
\hline
\end{tabular}

* Westcott factor for capture cross section.

** Integrated from $0.5 \mathrm{eV}$ to $100 \mathrm{keV}$ with $1 / \mathrm{E}$ spectrum. 
Table 8. Average Resonance Parameters for the Unresolved Resonance Region $\left({ }^{101} \mathrm{Ru}\right)$

\begin{tabular}{|c|c|c|c|c|c|c|c|}
\hline \multirow[b]{2}{*}{ Quantity } & \multirow[b]{2}{*}{ Unit } & \multirow{2}{*}{$\begin{array}{c}\text { BNL } \\
\text { [Mu81] }\end{array}$} & \multirow[b]{2}{*}{ ENDF/VI } & \multirow[b]{2}{*}{ JEF-2.2 } & \multirow{2}{*}{$\begin{array}{c}\text { JENDL- } \\
3.2^{*}\end{array}$} & \multicolumn{2}{|c|}{ Present } \\
\hline & & & & & & $\begin{array}{c}\text { PT } \\
\text { Analysis }\end{array}$ & Adopted \\
\hline $\mathbf{R}^{\prime}$ & $\mathrm{fm}$ & & 5.617 & 6.90 & 5.062 & & 6.53 \\
\hline$\left\langle\mathrm{D}_{0}\right\rangle$ & $\mathrm{eV}$ & ** & 20.42 & 18.29 & 25.58 & $19.3 \pm 1.8$ & $19.3^{+}$ \\
\hline$S_{0}$ & $\times 10^{-4}$ & $0.54 \pm 0.04$ & 0.59 & 0.59 & 0.59 & $0.62 \pm 0.15$ & 0.62 \\
\hline$\left\langle\Gamma_{r_{0}}\right\rangle$ & $\mathrm{meV}$ & $180 \pm 15$ & 180 & 180.3 & 173 & & 183.7 \\
\hline$\left\langle D_{1}\right\rangle$ & eV & & 8.75 & 9.53 & 12.79 & & $9.65^{+}$ \\
\hline$S_{1}$ & $\times 10^{-4}$ & $6.1 \pm 0.4$ & 6.00 & 6.10 & 6.10 & & 6.10 \\
\hline$\left\langle\Gamma_{\gamma 1}\right\rangle$ & $\mathrm{meV}$ & & 180 & 190.4 & 173 & & 183.7 \\
\hline$\left\langle\mathrm{D}_{2}\right\rangle$ & $\mathrm{eV}$ & & 6.30 & 6.76 & 8.53 & & $6.43^{+}$ \\
\hline$S_{2}$ & $\times 10^{-4}$ & & 2.00 & 1.12 & 0.53 & & 1.20 \\
\hline$\left\langle\Gamma_{\gamma_{2}}\right\rangle$ & $\mathrm{meV}$ & & 180 & 180.3 & 173 & & 183.7 \\
\hline
\end{tabular}

* Average parameters at the low energy $(1.06 \mathrm{keV})$ of the unresolved region.

** mean observed level spacing $=16 \pm 2 \mathrm{eV}$.

+value at the neutron separation energy of ${ }^{102} \mathrm{Ru}$. 


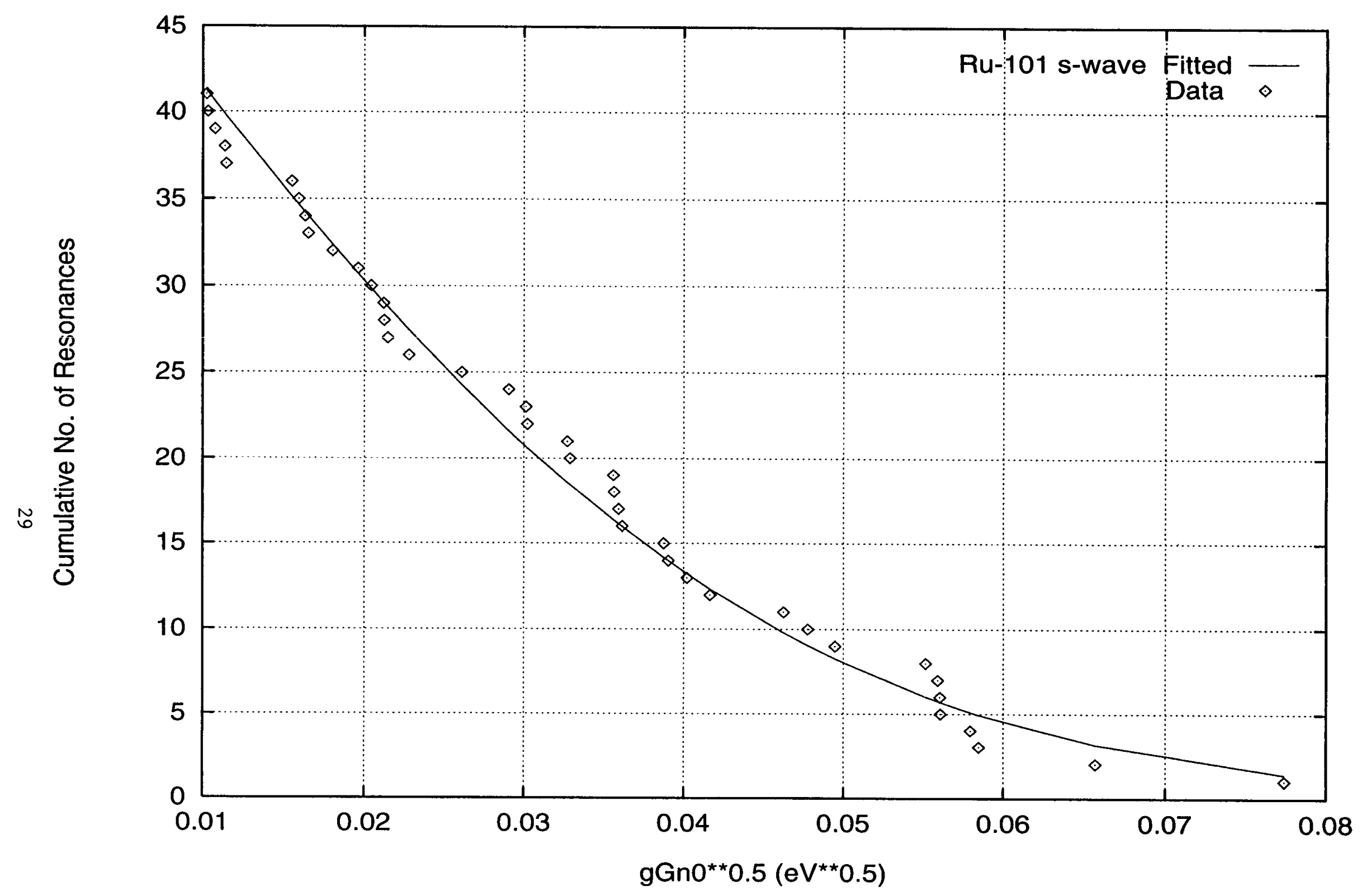

Fig. 9. Complement of the Cumulative Distribution of Neutron Reduced Widths ( ${ }^{101} \mathrm{Ru}, \mathrm{s}$-wave) 


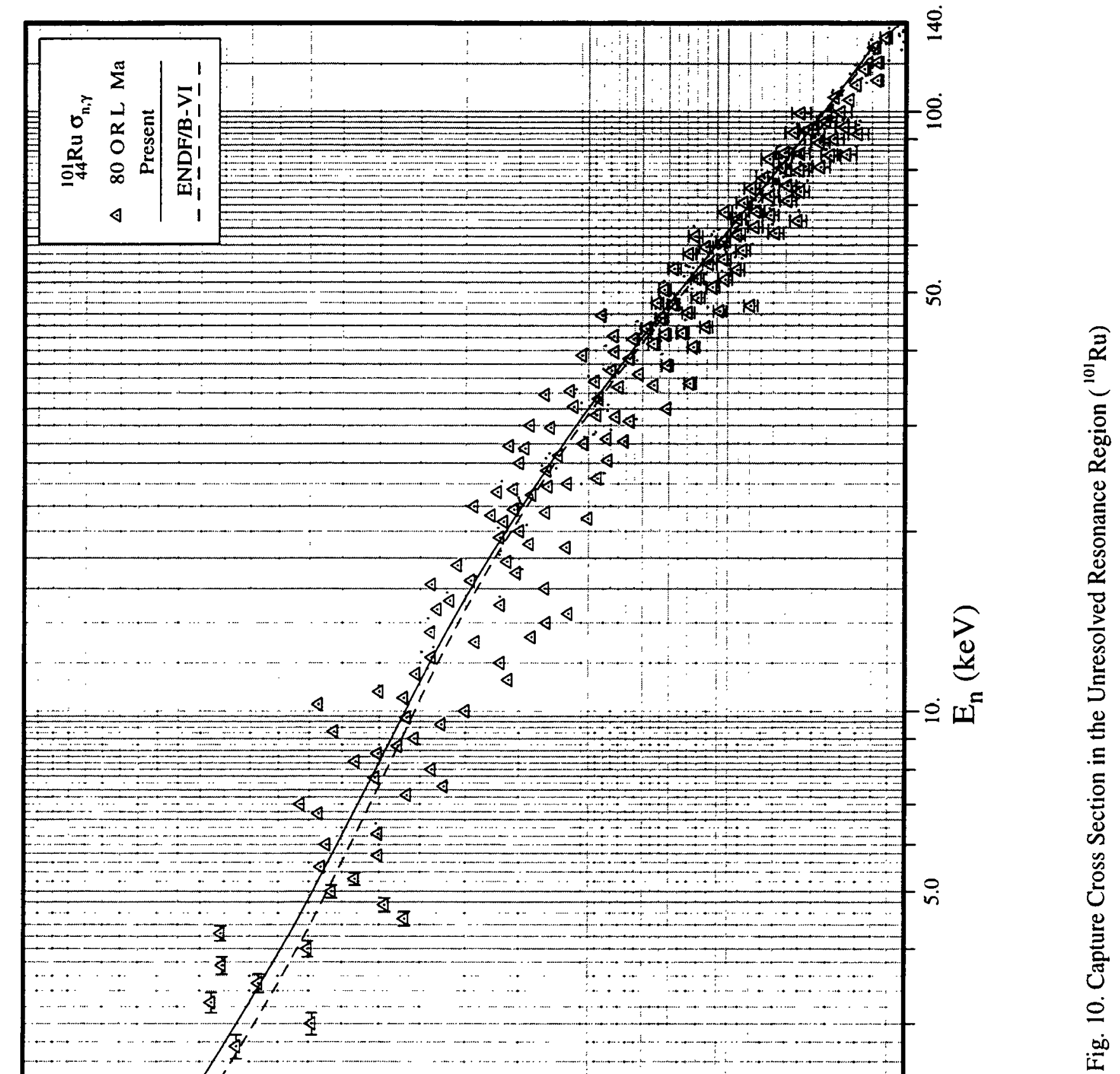


II.D. ${ }^{103} \mathbf{R h}$

1. Thermal Region

Known positive-energy neutron resonances account for the thermal capture cross section [Mus1], but not the bound coherent scattering length, $b^{\prime}=5.88 \pm 0.04$ fm, reported by Koester [Ko91]. On this basis, a bound level is postulated to account for the discrepancy in the coherent scattering length. This is achieved by decreasing the radiative width of the first resonance at $1.259 \mathrm{eV}$ from $154 \mathrm{meV}$ to $151 \mathrm{meV}$. This provides about 2.25 barns of capture contribution for the bound level. With this condition, the parameters of a bound level are determined as: $\mathrm{E}_{0}=\mathbf{- 5 5}$ $\mathrm{eV}, \Gamma_{\mathrm{n}}=101 \mathrm{meV}$, and $\Gamma_{\gamma}=160 \mathrm{meV}$. In this calculation, an effective scattering radius of 6.56 $\mathrm{fm}$ was adopted from the work of Ribon [Ri70]. The Westcott factor for capture is calculated as 1.0229. The thermal characteristics are summarized in Table 9 and are compared with other evaluations.

\section{Resolved resonance parameters}

Transmission data of Ribon [Ri70] at Saclay covered the energy region up to $4140 \mathrm{eV}$. In addition, the capture data of Carlson [Ca7lb] covered the energy region up to $989 \mathrm{eV}$. Macklin [Ma80] reported parameters from capture measurements in the energy region from 2644 to $4163 \mathrm{eV}$. The upper limit of the present resolved resonance region is set to $4.116 \mathrm{keV}$.

According to Macklin [Ma80], there is a problem with Ribon's energy scale [Ri70]. In this evaluation, correlation analysis between Macklin's energies and those of Ribon show that the time of flights are related by:

$$
t=0.99906891+6.0584518 \times 10^{-5} t_{\text {Riz } \overline{0}}
$$

We note that Macklin's $\mathrm{g} \Gamma_{\mathrm{n}}$ values are about 1.5 times larger than those of Ribon in the overlapping energy regions. On the other hand, there is good agreement between Ribon's and Carlson's $\mathrm{g} \Gamma_{\mathbf{n}}$ values. Because of these considerations, Macklin's scattering widths are not included in the present evaluation. This is additionally supported by the observation that the derived average parameters, $S_{0}, S_{1}$, and $S_{2}$ [Ma80] are about a factor 1.5 larger than the corresponding values obtained from the systematics studies [Mu81]. However, we adopted Macklin's energy scale.

The $l$ assignments were performed by the Bayesian method. For all observed resonances, the resulting cumulative Porter-Thomas distributions are displayed in Fig. 11. For s- and p-wave resonances, the fits are shown in Fig. 12 and Fig. 13, respectively. The average resonance parameters for s- and p-wave resonances, derived from this analysis, are summarized in Table 10 and are compared with [Mu81], as well as other evaluations.

\section{Unresolved Resonance Parameters}

Since the first excited level of ${ }^{103} \mathrm{Rh}$ is $39.756 \mathrm{keV}$, the upper limit of the unresolved resonance region is set to $40.15 \mathrm{keV}$. 
With the exception of minor adjustments in the s-wave radiative width and the p-wave strength function, the average resonance properties, obtained from the analysis of the resolved energy region, were adopted. The level spacing is assumed to vary with energy and spin according to the Gilbert-Cameron's level density formula; the associated parameters [Mu98a] and spin dispersion parameter of 3.1 were adopted from [Mu98b]. The average p-wave radiative width and the d-wave average parameters, assumed in this evaluation, are based on the systematics investigation [Mu84]. The capture cross section in the unresolved energy region, generated on the basis of the present average parameters, listed in the last column of Table 10, is shown in Fig. 14. In the present evaluation, the capture data by Wisshak et al. at Karlsruhe [Wi90] and Bokhovko at Obninsk [Bo85] were considered. Earlier measurements were reported in the curve book of McLane et al. [Mc88]. The calculated capture cross section of the present study shows good agreement with the recent data of [Wi90] up to $100 \mathrm{keV}$.

Maxwellian average capture cross section for a temperature of $30 \mathrm{keV}$ for the energy region $10^{-5} \mathrm{eV}$ to $225 \mathrm{keV}$ was computed as $809 \mathrm{mb}$. This is in excellent agreement with a value of $810 \pm 15 \mathrm{mb}$ [Be92].

Table 9. Thermal Characteristics $\left({ }^{103} \mathrm{Rh}\right)$

\begin{tabular}{|c|c|c|c|c|c|c|}
\hline Quantity & Unit & $\begin{array}{c}\text { BNL } \\
{[\mathrm{Mu} 81]}\end{array}$ & $\begin{array}{c}\text { ENDF/B- } \\
\text { VI }\end{array}$ & JEF-2.2 & JENDL-3.2 & Present \\
\hline$R^{\prime}$ & $\mathrm{fm}$ & $6.2 \pm 0.3$ & 7.09 & 6.20 & 6.20 & 6.56 \\
$b^{\prime}$ & $\mathrm{fm}$ & $5.0 \pm 0.1$ & 6.13 & & & 5.88 \\
\hline$\sigma_{\gamma}$ & barns & $145 \pm 2$ & 147 & 146 & 147 & 145 \\
$\sigma_{\mathrm{s}}$ & barns & & 4.69 & 3.43 & 3.28 & 4.37 \\
\hline RI-capt. & barns & $1100 \pm 50$ & 1035 & 1035 & 1040 & 1036 \\
\hline
\end{tabular}


Table 10. Average Resonance Parameters in Unresolved Resonance Region $\left({ }^{103} \mathrm{Rh}\right)$

\begin{tabular}{|c|c|c|c|c|c|c|c|}
\hline \multirow{2}{*}{ Value } & \multirow{2}{*}{ Unit } & \multirow{2}{*}{$\begin{array}{c}\text { ENDF/B- } \\
\text { VI }\end{array}$} & \multirow{2}{*}{ JEF-2.2 } & \multirow{2}{*}{$\begin{array}{c}\text { JENDL- } \\
3.2^{\mathrm{a}}\end{array}$} & \multirow{2}{*}{$\begin{array}{c}\text { BNL } \\
\text { [Mu81] }\end{array}$} & \multicolumn{2}{|c|}{ Present } \\
\hline & & & & & & $\begin{array}{c}\text { PT } \\
\text { Analysis } \\
\end{array}$ & Adopted \\
\hline$R^{\prime}$ & fm & 7.09 & $\begin{array}{c}6.20^{b} \\
(6.56)^{c}\end{array}$ & $\begin{array}{c}6.20^{\mathrm{b}} \\
(6.521)^{\mathrm{c}}\end{array}$ & $6.2 \pm 0.3$ & & 6.56 \\
\hline$<D_{0}>$ & $\overline{\mathrm{eV}}$ & 10.12 & 25.77 & $32.13^{+}$ & $16 \pm 1$ & $28.6 \pm 1.6$ & $28.6^{d}$ \\
\hline$S_{0}$ & $\times 10^{-4}$ & 0.43 & 0.485 & 0.445 & $0.53 \pm 0.05$ & $0.57 \pm 0.09$ & 0.57 \\
\hline$\left\langle\Gamma_{r 0}\right\rangle$ & $\mathrm{meV}$ & 153 & 161.25 & 230 & $160 \pm 15$ & & 170 \\
\hline$\left\langle D_{1}\right\rangle$ & $\mathrm{eV}$ & 13.5 & 11.86 & $14.28^{+}$ & & $14.5 \pm 0.6$ & $14.3^{d}$ \\
\hline$S_{1}$ & $\times 10^{-4}$ & 5.6 & 6.332 & 4.15 & $5.5 \pm 0.9$ & $5.0 \pm 0.6$ & 4.81 \\
\hline$\left\langle\Gamma_{n 1}\right\rangle$ & $\mathrm{meV}$ & 153 & 161.25 & 230 & & & 150 \\
\hline$\left\langle D_{2}\right\rangle$ & $\mathrm{eV}$ & & 7.52 & $8.57^{+}$ & & & $9.48^{d}$ \\
\hline$S_{2}$ & $\times 10^{-4}$ & & 0.595 & 0.53 & & & 1.0 \\
\hline$\left.<\Gamma_{y 2}\right\rangle$ & $\mathrm{meV}$ & & 161.25 & 230 & & & 140 \\
\hline
\end{tabular}

a) Value at the low energy $(3.58 \mathrm{keV})$ of the unresolved resonance region.

b) For the resolved energy region.

c) For the unresolved energy region.

d) Value at the neutron separation energy of ${ }^{104} \mathrm{Rh}$. 


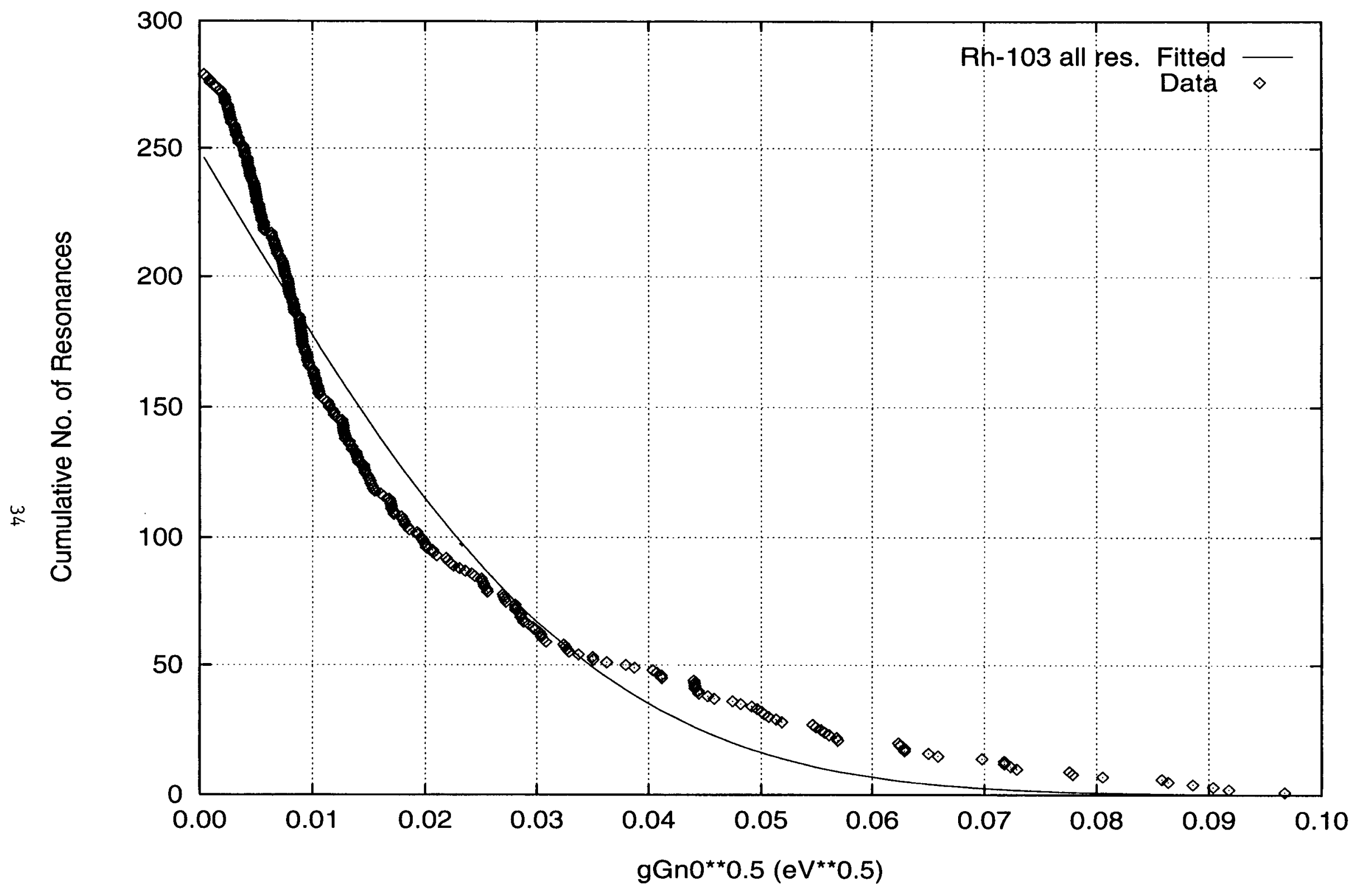

Fig. 11. Complement of the Cumulative Distribution of Neutron Reduced Widths $\left({ }^{103} \mathrm{Rh}\right)$. All resonances were assumed as s-wave. 


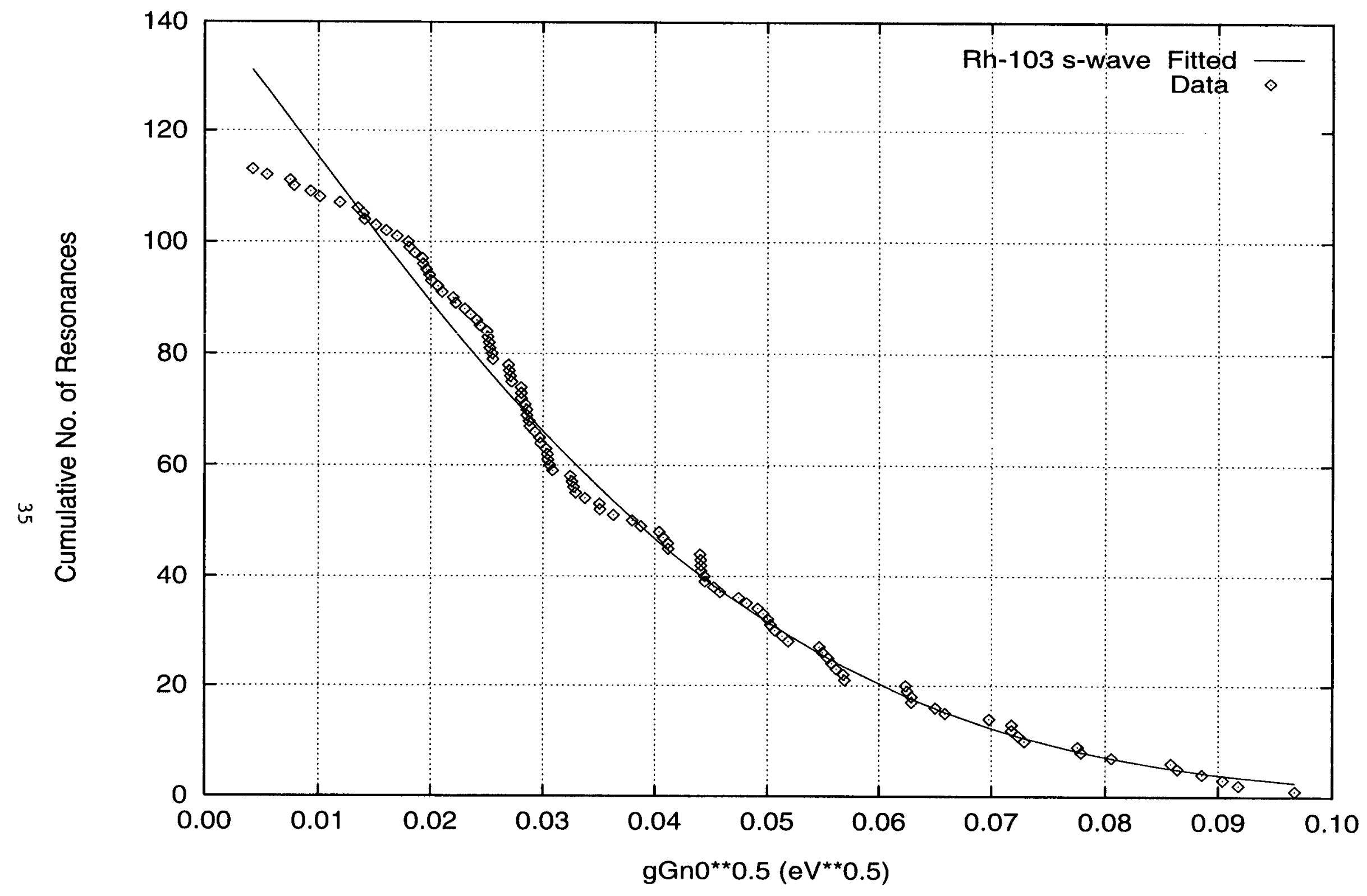

Fig. 12. Complement of the Cumulative Distribution of Neutron Reduced Widths ( ${ }^{103} \mathrm{Rh}, \mathrm{s}$-wave) 


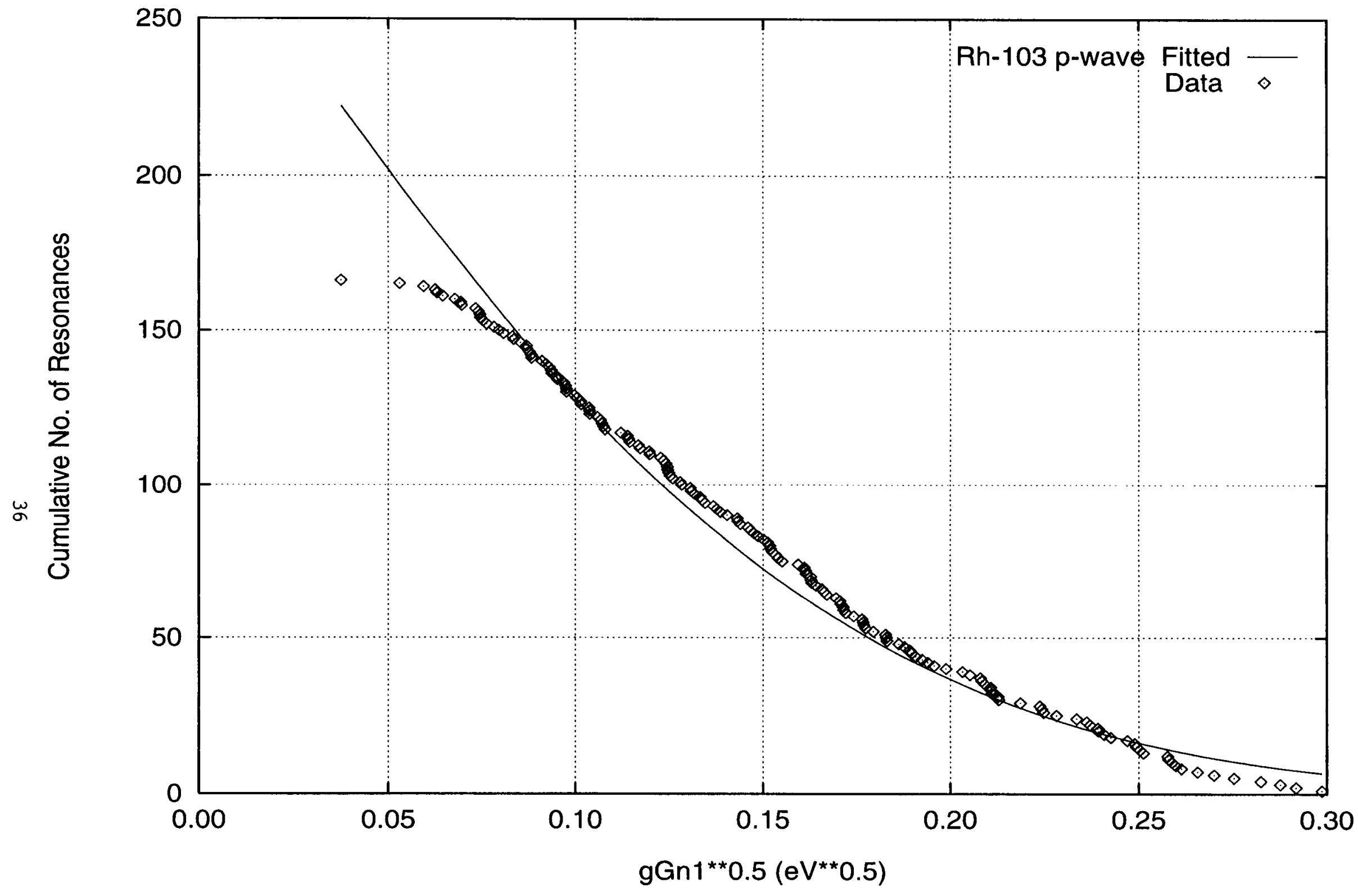

Fig. 13. Complement of the Cumulative Distribution of Neutron Reduced Widths $\left({ }^{103} \mathrm{Rh}, \mathrm{p}\right.$-wave) 


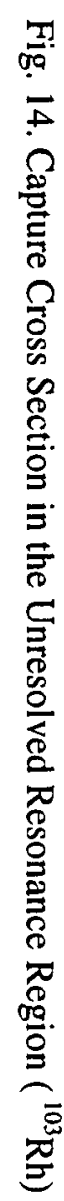

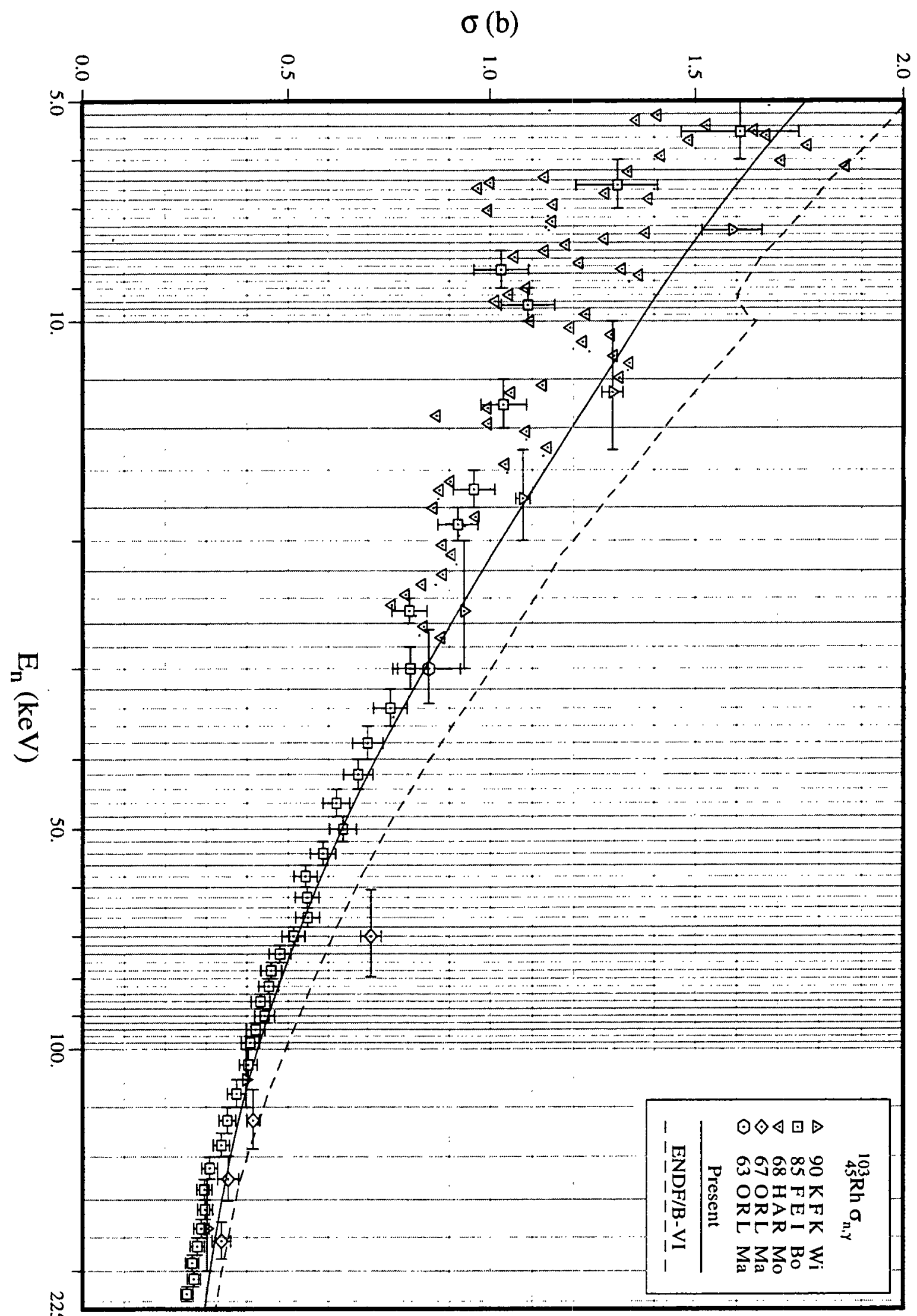


III.E. ${ }^{105} \mathbf{P d}$

\section{Thermal Region}

An evaluated thermal capture cross section of ${ }^{105} \mathrm{Pd}, \sigma_{\gamma}{ }^{0}=20.8 \mathrm{~b}$, was obtained by taking a weighted average of $22.0 \pm 1.5 \mathrm{~b}$ [Bu82] and $20.4 \pm 0.9 \mathrm{~b}$. The latter was derived by subtracting the isotopic contributions from a value of $6.6 \pm 0.1 \mathrm{~b}$ for the natural element [An80]. Since the contribution of positive energy resonances to the thermal capture cross section at $0.0253 \mathrm{eV}$ is only $2.3 \mathrm{~b}$, one or more s-wave bound levels are required to fit the thermal capture cross section. An examination of the gamma-ray spectra of thermal neutrons [Or70] indicates that two bound levels with spins 2 and 3 and about equal contribution to the thermal capture cross section are required. As a starting point in the fitting procedure, we adopted Glaettli et al.'s [G187] incoherent scattering length, $b_{+}-b_{-}=-5.2 \pm 3.8 \mathrm{fm}$, and a coherent scattering length, $b=7.3 \mathrm{fm}$, by the subtraction method. However, with these values it was not possible to obtain consistent results. Final adjustments, principally in the incoherent scattering length, had to be made. The bound level parameters are presented in Table 11. With these parameters, $\sigma_{\text {coh }}=7.263 \mathrm{~b}$ $(b=7.61 \mathrm{fm}), \sigma_{\text {inc }}=0.052 \mathrm{~b}\left(\mathrm{~b}_{+}-\mathrm{b}_{-}=-1.3 \mathrm{fm}\right)$, and $\sigma_{\gamma}{ }^{0}=20.9 \mathrm{~b}$. Comparisons of the thermal cross sections and resonance integrals of the present evaluation with those of ENDF/B-VI, JEF-2 and JENDL-3 are summarized in Table 12. Note the large difference between the present evaluation of the scattering cross section and those in the other evaluations.

\section{Resolved Resonance Parameters}

Resonance parameters measured by Staveloz et al. [St79] were adopted in this evaluation. These preliminary results were the main source in Mughabghab's BNL compilation in 1981 [Mu81]. Of a total of 198 positive-energy resonances, 141 resonances were assigned as s-wave and 57 as p-wave on the basis of the Bayesian analysis. Uncertainty-weighted averaging of 70 radiative widths, which were reported by Staveloz et al. [St79], resulted in an average value of $148 \pm 4 \mathrm{meV}$; this value was assumed for the remaining resonances. Resonance spins were assigned randomly to those resonances for which the spin had not been determined. This random assignment assumes that the level density is proportional to $(2 \mathrm{~J}+1)$.

The distributions of s- and p-wave reduced neutron widths were analyzed by fitting to a Porter-Thomas distribution. For the s-wave case, resonances with reduced widths smaller than $0.08 \mathrm{meV}$ were excluded in the fitting procedure to minimize the $\chi^{2}$ value. In Fig. 15, the fit to the data resulted in $\left\langle\mathrm{D}_{0}\right\rangle=10.32 \pm 0.47 \mathrm{eV}$ and $\left\langle\mathrm{g} \Gamma_{\mathrm{n}}{ }^{0}\right\rangle=0.68 \pm 0.08 \mathrm{meV}$. These values yield swave strength function of $S_{0}=0.66 \pm 0.08$. The present $\left\langle D_{0}\right\rangle$ is consistent with values reported by

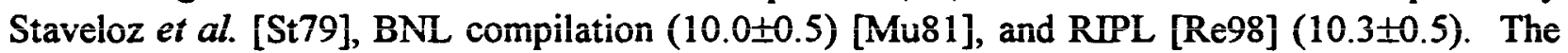
present $S_{0}$ is consistent with those reported in the BNL compilation $(0.6 \pm 0.1)$, RIPL $(0.60 \pm 0.05)$, and by Staveloz et al. $(0.63 \pm 0.07)$. In addition, the possible deviation from a Porter-Thomas distribution with one degree of freedom was investigated. Pandita and Agrawal [Pa92] calculated $v$ as 1.69 for this nuclide and suggested that intermediate structure is a possible reason for a deviation from a Porter-Thomas distribution $(v=1)$. However, as depicted in Fig. 16, concentration of strength in the considered energy region was not observed. Even though the fitting with $v=2$ (Fig. 17) seems slightly better than that for $v=1$, the latter was adopted in the 
present evaluation. For the p-wave population, resonances for which the reduced widths are smaller than $15 \mathrm{meV}$ were excluded in the fitting (Fig. 18). This procedure resulted in $\left\langle D_{1}\right\rangle=5.07 \pm 0.76 \mathrm{eV}$ and $S_{1}=4.56 \pm 1.00$. This $p$-wave level spacing is consistent with other evaluations. The derived $p$-wave strength function is also consistent, within the associated uncertainty range, with values of 5.8 \pm 0.3 in the BNL compilation [Mu81] and $4.1 \pm 0.4$ of Koester [Ko87].

\section{Unresolved Resonance Parameters}

The present unresolved energy region extends to $283.2 \mathrm{keV}$, which corresponds to the threshold energy of inelastic neutron scattering to the first excited level of ${ }^{105} \mathrm{Pd}$ at $280.51 \mathrm{keV}$. In this region, average capture cross sections measured by Macklin et al. [Ma81, Ma79], and Cornelis et al. [C082] were taken into consideration. These two data sets show good agreement with each other.

For this isotope, s-, p- and d-wave average resonance parameters were provided. Since the upper energy is relatively high, $d$-wave contribution is not negligible. The level spacing is assumed to vary with energy according to Gilbert-Cameron level density formula in which the associated parameters were adopted from Mughabghab and Dunford [Mu98a]. From the $(2 \mathrm{~J}+1)$ dependence of level density, spacings of $p$ - and d-wave resonances were assumed to be $1 / 2$ and $1 / 3$ of the s-wave spacing, respectively. In the calculation of the capture cross-section, the following parameters were initially applied: 1) $\left\langle\mathrm{D}_{0}\right\rangle$ and $\mathrm{S}_{0}$ values which were deduced from the resolved resonances; 2) $S_{1}$ which was adopted from the BNL compilation; 3) $S_{2}$ which was assumed to be the same as $S_{0}$; and 4) a $\left\langle\Gamma_{\gamma}\right\rangle$ of $148 \mathrm{meV}$ for s-, p- and d-waves. These parameters resulted in a calculated cross section, which is larger by about $15 \%$ at the low energy region and lower by a few \% at the high region than the measurements. Subsequently, to obtain good agreement with the measurements, $S_{0}$ and $S_{2}$ were adjusted to the values listed in Table 13. The resulting calculated capture cross section is shown in Fig. 19.

Maxwellian-averaged capture cross section for a temperature of $30 \mathrm{keV}$ was computed as $1.19 \mathrm{~b}$. This value is in excellent agreement with $1.20 \pm 0.06 \mathrm{~b}$ [Be92] and $1.19 \mathrm{~b}$ in the BNL compilation [Mu81].

Table 11. Bound Level Resonance Parameters $\left({ }^{105} \mathrm{Pd}\right)$

\begin{tabular}{|c|c|c|c|}
\hline Energy (eV) & $l$ and Resonance Spin & $\begin{array}{c}\text { Scattering Width } \\
(\mathrm{meV})\end{array}$ & $\begin{array}{c}\text { Capture Width } \\
(\mathrm{meV})\end{array}$ \\
\hline-29.64 & $l=0, \mathrm{~J}=2.0$ & 173.0 & 148.0 \\
-15.84 & $l=0, \mathrm{~J}=3.0$ & 25.68 & 148.0 \\
\hline
\end{tabular}


Table 12. Thermal Characteristics $\left({ }^{105} \mathrm{Pd}\right)$

\begin{tabular}{|c|c|c|c|c|c|c|c|}
\hline Quantity & Unit & $\begin{array}{c}\text { BNL } \\
{[\mathrm{Mu81}]}\end{array}$ & $\begin{array}{c}\text { 98CRC } \\
{[\mathrm{Ho98}]}\end{array}$ & $\begin{array}{c}\text { ENDF/ } \\
\text { B-VI }\end{array}$ & JEF-2.2 & $\begin{array}{c}\text { JENDL- } \\
3.2\end{array}$ & Present \\
\hline R' & fm & $6.6 \pm 0.3$ & & 6.60 & 6.695 & 7.05 & 6.60 \\
\hline $\begin{array}{c}\sigma_{\gamma}^{0} \\
\sigma_{\mathrm{s}}{ }^{0}\end{array}$ & $\begin{array}{c}\text { barn } \\
\text { barn }\end{array}$ & $\begin{array}{r}20.0 \pm 3.0 \\
5.0 \pm 0.6\end{array}$ & $22 \pm 2$ & $\begin{array}{c}20.1 \\
5.01\end{array}$ & $\begin{array}{c}21.8 \\
5.22\end{array}$ & $\begin{array}{c}20.3 \\
5.13\end{array}$ & $\begin{array}{c}20.9 \\
7.36\end{array}$ \\
\hline $\mathrm{gw}^{*}$ & & & & 0.9973 & 0.9951 & 0.9986 & 0.9995 \\
\hline $\begin{array}{c}\text { RI- } \\
\text { capt. } \\
\text { RI-total"* }\end{array}$ & $\begin{array}{l}\text { barn } \\
\text { barn }\end{array}$ & 62.2 & $60 \pm 20$ & $\begin{array}{c}111 \\
200\end{array}$ & $\begin{array}{c}93.1 \\
175\end{array}$ & $\begin{array}{c}96.8 \\
168\end{array}$ & $\begin{array}{c}95.2 \\
186\end{array}$ \\
\hline
\end{tabular}

* Westcott factor for capture cross section.

** Integrated from $0.5 \mathrm{eV}$ to $100 \mathrm{keV}$ with $1 / \mathrm{E}$ spectrum.

Table 13. Average Resonance Parameters for the Unresolved Resonance Region $\left({ }^{105} \mathrm{Pd}\right)$

\begin{tabular}{|c|c|c|c|c|c|c|c|}
\hline \multirow[b]{2}{*}{ Quantity } & \multirow[b]{2}{*}{ Unit } & \multirow{2}{*}{$\begin{array}{c}\text { BNL } \\
\text { [Mu81] }\end{array}$} & \multirow{2}{*}{$\begin{array}{c}\text { ENDF/ } \\
\text { B-VI }\end{array}$} & \multirow[b]{2}{*}{ JEF-2.2 } & \multirow{2}{*}{$\begin{array}{c}\text { JENDL- } \\
3.2^{*}\end{array}$} & \multicolumn{2}{|c|}{ Present } \\
\hline & & & & & & $\begin{array}{c}\text { PT } \\
\text { Analysis }\end{array}$ & Adopted \\
\hline $\mathbf{R}^{\prime}$ & $\mathrm{fm}$ & $6.6 \pm 0.2$ & & 6.695 & 4.60 & & 6.6 \\
\hline $\begin{array}{c}\left\langle\mathrm{D}_{0}\right\rangle \\
\mathrm{S}_{0} \\
\left\langle\mathrm{~T}_{\gamma 0}\right\rangle\end{array}$ & $\begin{array}{c}\mathrm{eV} \\
\times 10^{-4} \\
\mathrm{meV}\end{array}$ & $\begin{array}{c}10.0 \pm 0.5 \\
0.6 \pm 0.1 \\
145 \pm 8\end{array}$ & & $\begin{array}{c}10.3 \\
0.58 \\
155\end{array}$ & $\begin{array}{r}5.30 \\
0.60 \\
145\end{array}$ & $\begin{array}{c}10.32 \pm 0.4 \\
7 \\
0.66 \pm 0.08\end{array}$ & $\begin{array}{c}10.3^{* *} \\
0.50 \\
148\end{array}$ \\
\hline $\begin{array}{c}\left\langle D_{1}\right\rangle \\
S_{1} \\
\left\langle\Gamma_{\gamma_{1}}\right\rangle\end{array}$ & $\begin{array}{c}\mathrm{eV} \\
\times 10^{-4} \\
\mathrm{meV} \\
\end{array}$ & $5.8 \pm 0.3$ & & $\begin{array}{r}5.16 \\
4.80 \\
155 \\
\end{array}$ & $\begin{array}{r}2.65 \\
5.80 \\
145\end{array}$ & $\begin{array}{l}5.07 \pm 0.76 \\
4.56 \pm 1.00\end{array}$ & $\begin{array}{l}5.16^{* *} \\
5.80 \\
148\end{array}$ \\
\hline $\begin{array}{c}\left\langle\mathrm{D}_{2}\right\rangle \\
\mathrm{S}_{2} \\
\left\langle\Gamma_{\gamma_{2}}\right\rangle \\
\end{array}$ & $\begin{array}{c}\mathrm{eV} \\
\times 10^{-4} \\
\mathrm{meV} \\
\end{array}$ & & & $\begin{array}{r}3.44 \\
0.60 \\
155 \\
\end{array}$ & $\begin{array}{l}1.77 \\
0.98 \\
145\end{array}$ & & $\begin{array}{l}3.43^{* *} \\
0.80 \\
148\end{array}$ \\
\hline
\end{tabular}

* Average parameters at the low energy end $(2.049 \mathrm{keV})$ of the unresolved region.

** Value at the neutron separation energy of ${ }^{106} \mathrm{Pd}$. 


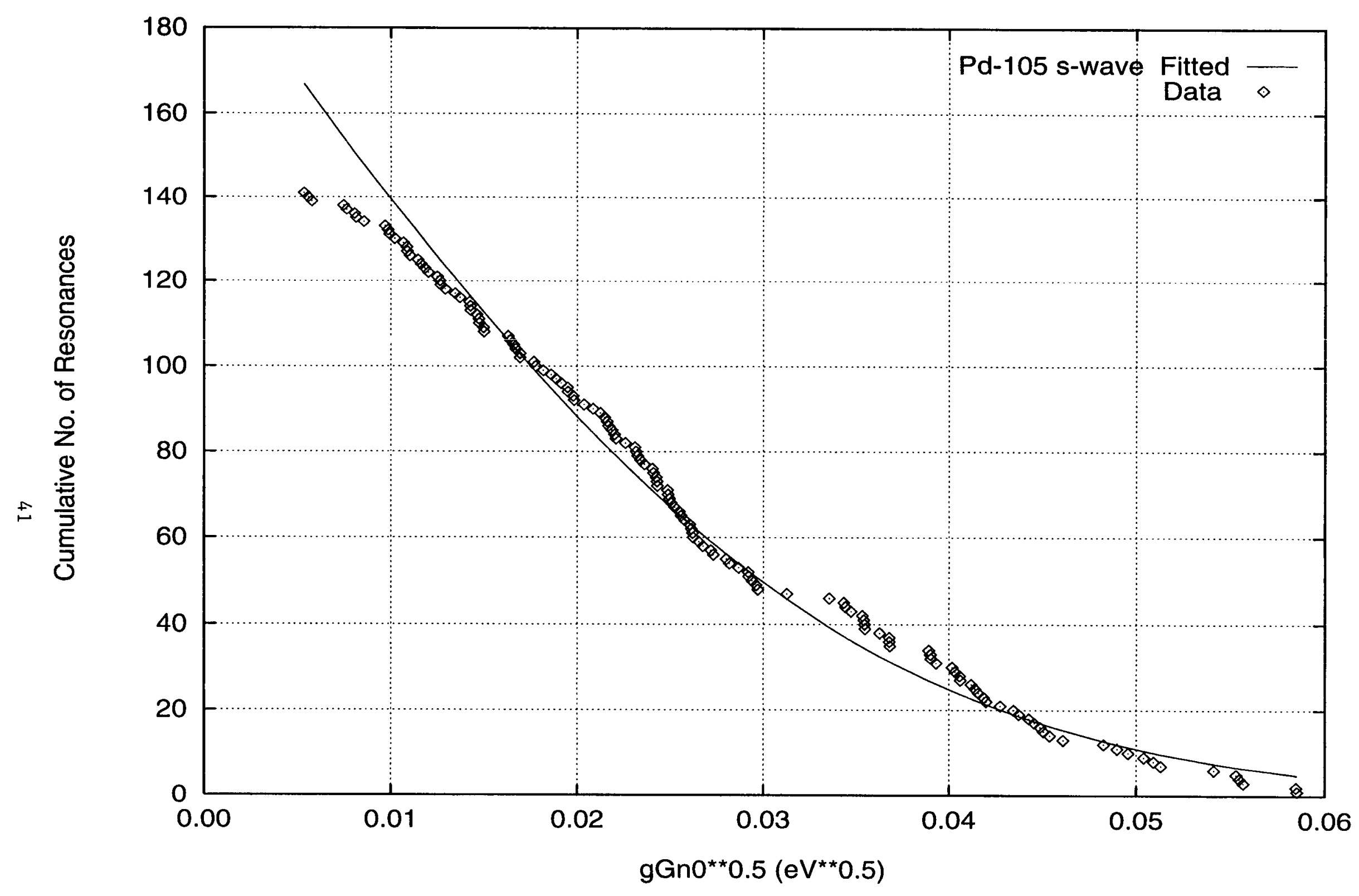

Fig. 15. Complement of the Cumulative Distribution of Neutron Reduced Widths ( ${ }^{105} \mathrm{Pd}, \mathrm{s}$-wave) 


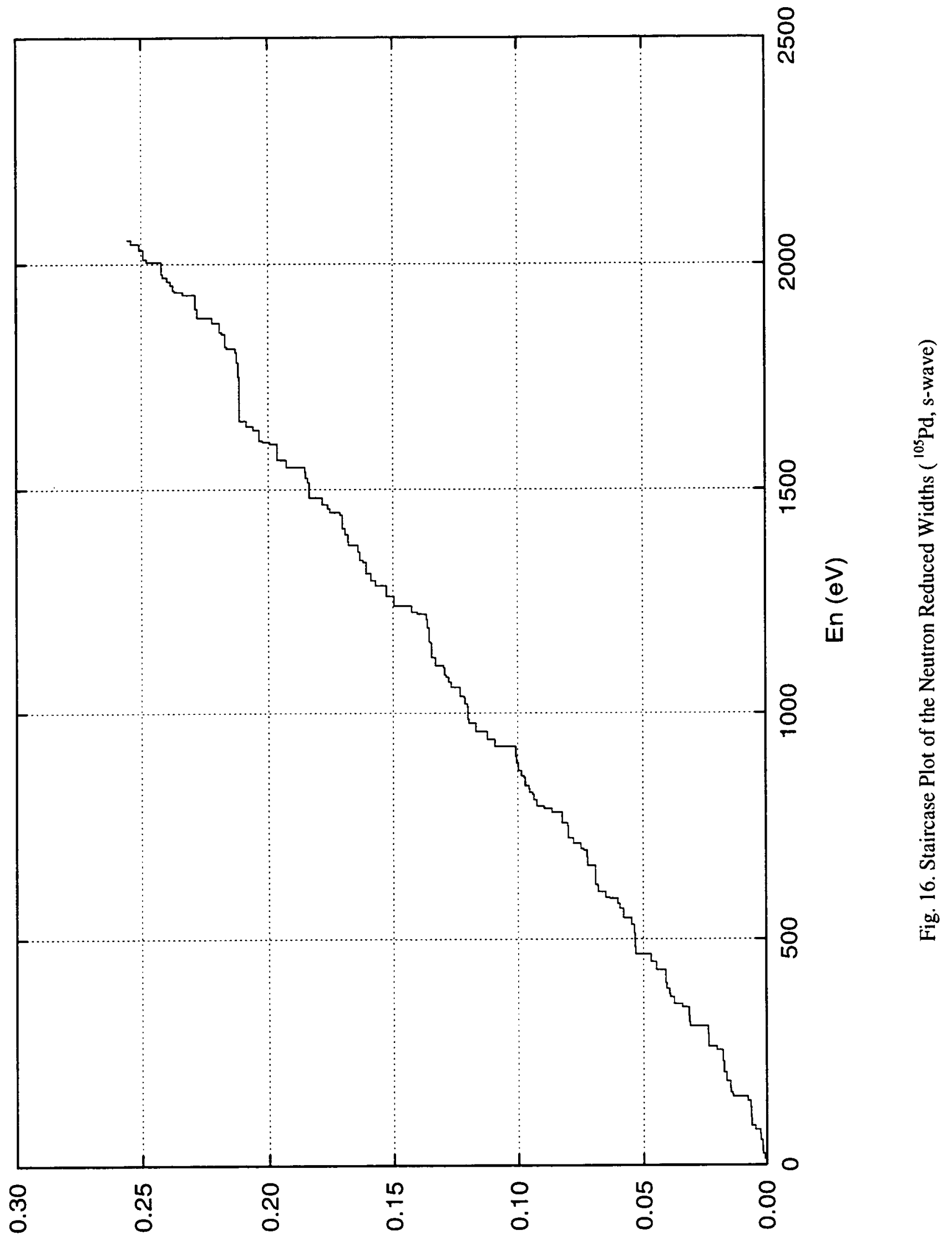

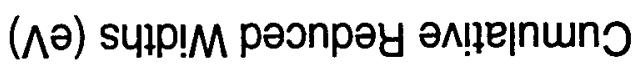




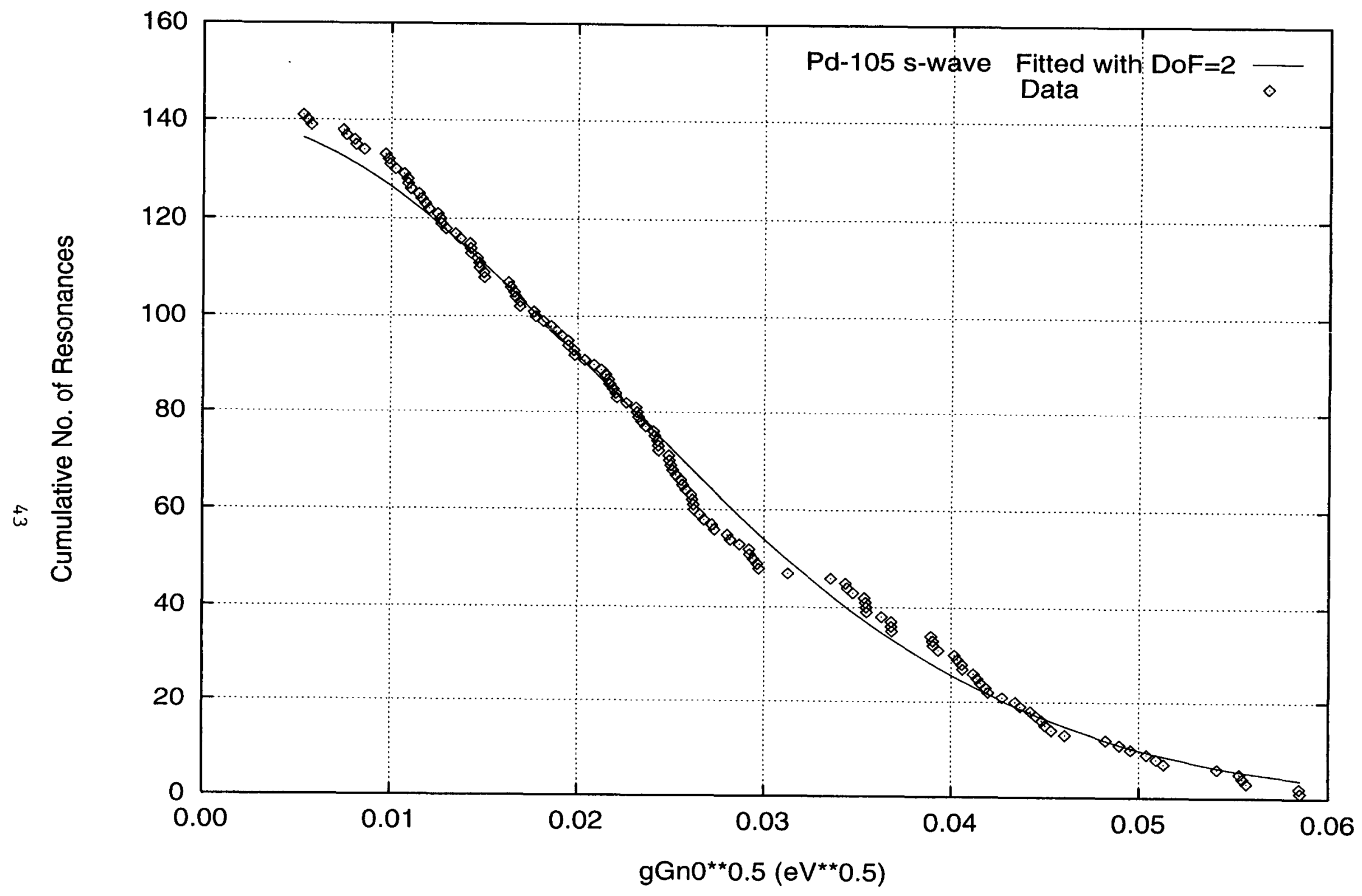

Fig. 17. Complement of the Cumulative Distribution of Neutron Reduced Widths ( ${ }^{105} \mathrm{Pd}, \mathrm{s}$-wave: fitted with the degree of freedom of 2 ) 


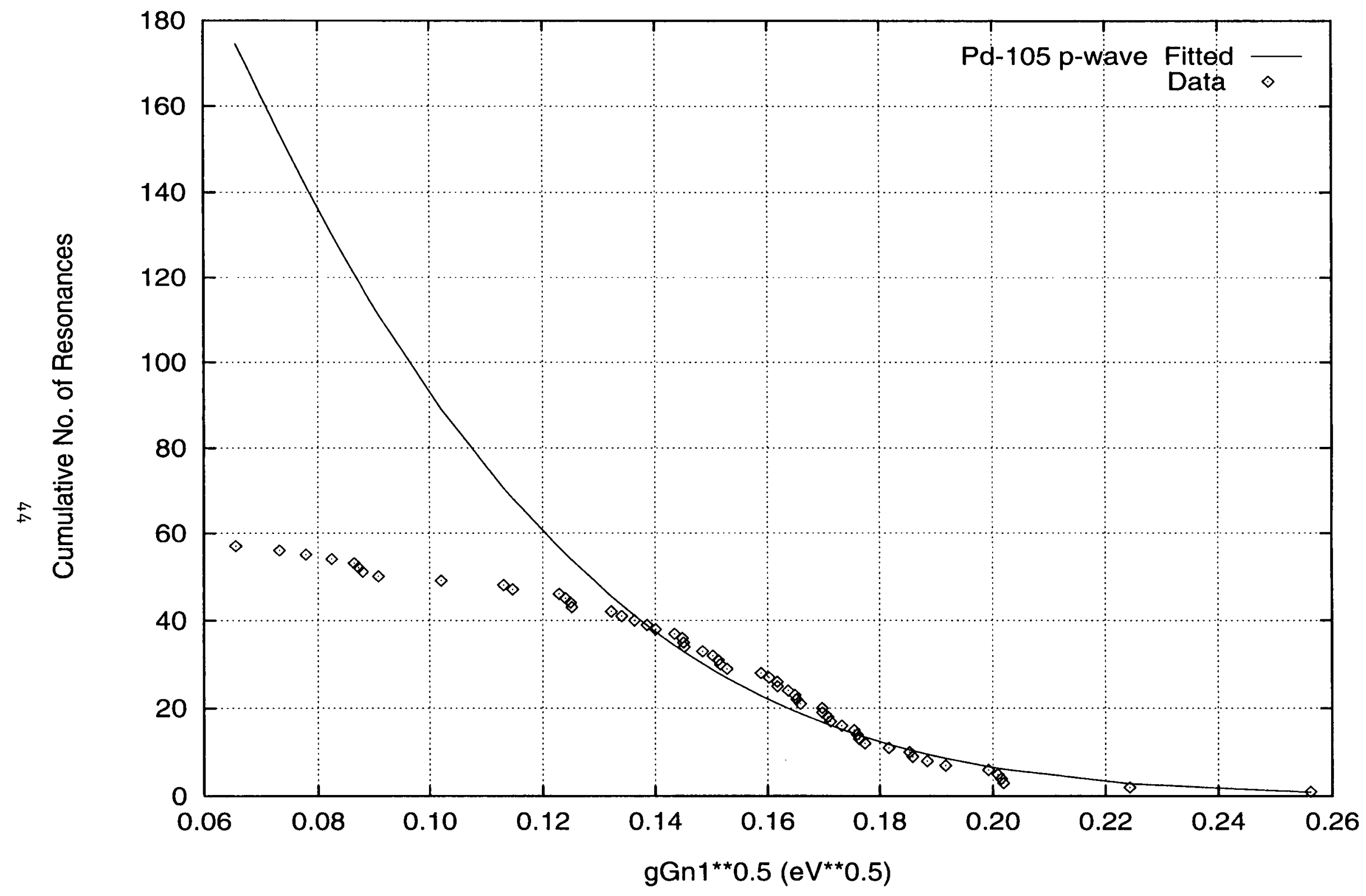

Fig. 18. Complement of the Cumulative Distribution of Neutron Reduced Widths ( ${ }^{105} \mathrm{Pd}, \mathrm{p}$-wave) 


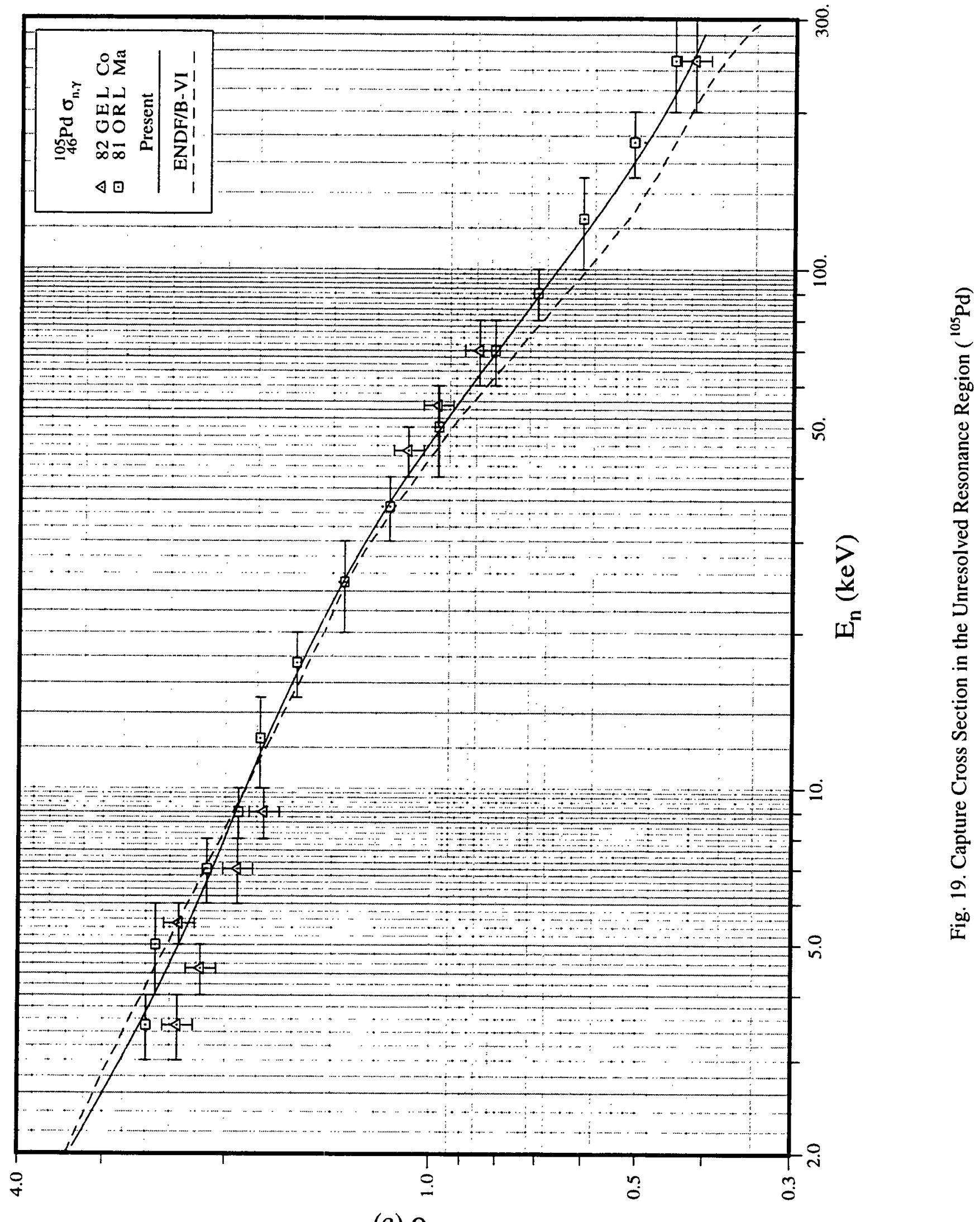

(q) $\mathrm{D}$ 


\section{III.F. ${ }^{109} \mathbf{A g}$}

\section{Thermal Region}

The resolved resonance parameters in the energy region from $5.9 \mathrm{eV}$ to $2.5 \mathrm{keV}$, as well as the effective scattering radius, $R^{\prime}=6.6 \mathrm{fm}$, were adopted from the BNL compilation [Mu81]. Since positive energy resonances account for the thermal capture cross section and the spindependent coherent scattering amplitudes, no bound levels are required. As shown in Table 14, only minor differences exist between the present thermal cross sections, those reported in the BNL compilation [Mu81], and existing evaluated libraries.

\section{Resolved Resonance Region}

To resolve a discrepancy in the existing cross section measurements of ${ }^{109} \mathrm{Ag}$, several new measurements, which reported resonance parameter information since the BNL compilation [Mu81], were carried out [Ma82], [Mi83], [Co97], and [L097]. These data were incorporated partially in the ENDF/B-VI revision and JENDL-3. In the present evaluation, the parameters of neutron resonances up to $2.506 \mathrm{keV}$ were basically adopted from the BNL compilation [Mu81]. Below this energy region, the spin assignments of Corvi et al. [Co97] and the neutron widths of Lowie et al. [L097] were adopted. The resonance parameters from $2.6 \mathrm{keV}$ to $5.0 \mathrm{keV}$ are based on the data of Macklin [Ma82b] and Mizumoto et al. [Mi83]. Even though individual resonance parameters were reported [Ma82b, Mi83] up to $7 \mathrm{keV}$, the upper energy region of the resolved energy region was set to $4.996 \mathrm{keV}$ in this evaluation. Above $5 \mathrm{keV}$, the cross sections are represented by average resonance parameters.

For weak resonances in the energy region from $2.5 \mathrm{keV}$ to $5.0 \mathrm{keV}$, the $\mathrm{g} \Gamma_{\mathrm{n}}$ values were calculated from the reported capture areas [Ma82b] for an assumed spin statistical factor, $g$, and an assumed constant radiative width of $143 \mathrm{meV}$. In this procedure, consistency, within the quoted uncertainties, was achieved with the parameters of Mizumoto et al. [Mi83]. For strong resonances, the scattering widths of Mizomoto et al. were adopted. In some favorable cases, the capture areas [Ma82b] were combined with the transmission data [Mi83] to deduce spins of resonances. Of a total number of 307 resonances, 236 were assigned as $l=0$ and the remaining as $l=1$ by Bayesian analysis. Resonance spins were assigned randomly to those resonances for which the spin is not reported in the BNL compilation [Mu81], Lowie et al. [Lo97], or determined in the present analysis. The weighted-average value of the radiative widths of $21 \mathrm{~s}-$ wave resonances was calculated as $129.9 \pm 2.6 \mathrm{meV}$, which is in very good agreement with that in the BNL compilation (130 meV) [Mu81] and RIPL (130 $\pm 20 \mathrm{meV})$ [Re98].

The distributions of reduced neutron widths for $s$ - and $p$-wave resonances were analyzed in terms of the Porter-Thomas distribution. For the s-wave case, those resonances with reduced neutron widths less than $0.4 \mathrm{meV}$ were excluded in order to obtain a good fit. The result of the analysis, depicted in Fig. 20, shows that there is a surplus of s-wave resonances with reduced widths in the range of $0.1 \mathrm{meV}-0.4 \mathrm{meV}$, possibly belonging to the $l=1$ population. However, as shown in Fig. 21, the staircase plot suggests that there is a minor, if any, spurious p-wave resonances. The Porter-Thomas fitting resulted in $\left\langle\mathrm{D}_{0}\right\rangle=20.0 \pm 0.8 \mathrm{eV}$ and $\left\langle\mathrm{g} \Gamma_{\mathrm{n}}{ }^{0}\right\rangle=1.04 \pm 0.10$ meV. These values yield s-wave strength function of $S_{0}=0.52 \pm 0.05$. The present $\left\langle D_{0}\right\rangle$ value is 
consistent with $20 \pm 2 \mathrm{eV}$ [Mi83] but is much larger than the values, 14 $\pm 2 \mathrm{eV}$ [Mu81] and $15.1 \pm 1.4 \mathrm{eV}$ [Re98]. The present $S_{0}$ is in good agreement, within the uncertainty limits, with reported values: $0.46 \pm 0.15$ [Mu81], $0.45 \pm 0.05$ [Mi83], but not with $0.75 \pm 0.08$ [Re98]. For pwave resonances, the fitting analysis, with a cutoff of $11.5 \mathrm{meV}$ for the p-wave reduced width, resulted in $\left\langle D_{1}\right\rangle=10.5 \pm 2.1 \mathrm{eV}$ and $S_{1}=1.31 \pm 0.29$ (Fig. 22). Even though the level spacing seems to be reasonable, the p-wave strength function is quite low in this mass region $\left(S_{1}=3.8 \pm 0.6\right)$ [Mu81]. It may stem from the fact that some strong to medium-strong resonances, assigned as s-wave by the Bayesian analysis, may in fact be p-wave resonances.

\section{Unresolved Resonance Region}

In the unresolved energy region, $5 \mathrm{keV}-88.8 \mathrm{keV}$, the average capture cross section measured by Macklin [Ma82b] was adopted as the reference cross section. The cross section measurements of Bokhovko et al. [Bo87] and Mizumoto et al. [Mi83] show relatively good agreement with Macklin's measurements. Earlier measurements reported in the Neutron Cross Section Curves [Mc88] are discrepant with these three measurements. Since the d-wave strength function has a minimum value in this mass region [Mu81], the small $\mathrm{d}$-wave contribution to the capture cross section below $88 \mathrm{keV}$, was not included in the analysis of ${ }^{109} \mathrm{Ag}$. The average resonance parameters which were applied in the present calculation are listed in the last column of Table 15. We adopted $S_{0}$ value which was increased by its uncertainty to improve the fit. From the $(2 \mathrm{~J}+1) \cdot \exp \left\{-(\mathrm{J}+1 / 2)^{2} / 2 \sigma^{2}\right\}$ dependence of the level spacing, the average level spacing of $p$-wave is calculated as $1 / 2.005$ of the s-wave spacing for a spin dispersion parameter $\sigma=3.2$ [Mu98b]. The adopted average gamma width is obtained by weighting Macklin's value of $146 \pm 6 \mathrm{meV}$ [Ma82b] and a value of $129.9 \pm 2.6 \mathrm{meV}$ derived from resolved resonances. Figure 23 compares our calculated capture cross section with measurements [Bo87, Mi83, Ma82b], as well as the ENDF/B-VI evaluation.

Maxwellian-averaged capture cross section for a temperature of $30 \mathrm{keV}$ was computed as $787 \mathrm{mb}$. In extending the energy region up to $1 \mathrm{MeV}$, the capture cross-section in the region 88.8 $\mathrm{keV}$ to $1 \mathrm{MeV}$ was adopted from the ENDF/B-VI evaluation. The present computed cross section is to be compared with a value of $779 \pm 23 \mathrm{mb}$ [Be92]. 
Table 14. Thermal Characteristics $\left({ }^{109} \mathrm{Ag}\right)$

\begin{tabular}{|c|c|c|c|c|c|c|c|c|}
\hline Quantity & Unit & $\begin{array}{c}\text { BNL } \\
\text { [Mu81] }\end{array}$ & $\begin{array}{c}98 \mathrm{CR} \\
\mathrm{C} \\
{[\mathrm{Ho} 98]}\end{array}$ & $\begin{array}{l}\text { ENDF/ } \\
\text { B-VI }\end{array}$ & JEF-2.2 & $\begin{array}{c}\text { JENDL- } \\
3.2\end{array}$ & $\begin{array}{l}\text { LIPAR-5 } \\
\text { [Ab97] }\end{array}$ & Present \\
\hline $\mathbf{R}^{\prime}$ & $\mathrm{fm}$ & $6.6 \pm 0.2$ & & 6.60 & 6.60 & 7.05 & & 6.60 \\
\hline$\sigma_{\gamma}{ }^{0}$ & barn & $91.0 \pm 1.0$ & 91.2 & 91.0 & 90.8 & 90.5 & 90.7 & 90.8 \\
\hline$\sigma_{s}^{0}$ & barn & $2.55 \pm 0.06$ & & 2.25 & 2.30 & 2.48 & 2.42 & 2.12 \\
\hline $\mathrm{gw}^{*}$ & & & & 1.006 & 1.005 & 1.005 & 1.0050 & 1.0053 \\
\hline $\begin{array}{c}\text { RI- } \\
\text { capt. }\end{array}$ & barn & $1400 \pm 400$ & 1480 & 1471 & 1473 & 1 & 1467 & 1476 \\
\hline RI-total ${ }^{* *}$ & barn & & & 1691 & 1690 & 1689 & & 1691 \\
\hline
\end{tabular}

* Westcott factor for capture cross section.

** Integrated from $0.5 \mathrm{eV}$ to $100 \mathrm{keV}$ with $1 / \mathrm{E}$ spectrum.

Table 15. Average Resonance Parameters for the Unresolved Resonance Region $\left({ }^{109} \mathrm{Ag}\right)$

\begin{tabular}{|c|c|c|c|c|c|c|c|}
\hline \multirow[b]{2}{*}{ Quantity } & \multirow[b]{2}{*}{ Unit } & \multirow{2}{*}{$\begin{array}{c}\text { ENDF/ } \\
\text { B-VI }\end{array}$} & \multirow[b]{2}{*}{ JEF-2.2 } & \multirow{2}{*}{$\begin{array}{l}\text { JENDL- } \\
3.2^{*}\end{array}$} & \multirow{2}{*}{$\begin{array}{c}\text { BNL } \\
\text { [Mu81] }\end{array}$} & \multicolumn{2}{|c|}{ Present } \\
\hline & & & & & & $\begin{array}{c}\text { PT } \\
\text { Analysis }\end{array}$ & Adopted \\
\hline $\begin{array}{c}\mathbf{R}^{\prime} \\
\text { (URR) }\end{array}$ & $\mathrm{fm}$ & & 6.30 & 6.618 & $6.6 \pm 0.2$ & & 6.60 \\
\hline$\left\langle\mathrm{D}_{0}\right\rangle$ & $\mathrm{eV}$ & & 16.07 & 19.40 & $14 \pm 2$ & $20.0 \pm 0.8$ & $20.0^{* *}$ \\
\hline$S_{0}$ & $\times 10^{-4}$ & & 0.64 & 0.54 & $0.46 \pm 0.15$ & $0.52 \pm 0.05$ & 0.57 \\
\hline$\left\langle\Gamma_{\gamma 0}\right\rangle$ & $\mathrm{meV}$ & & 132.3 & 130 & 130 & & 133 \\
\hline$\left\langle\mathrm{D}_{1}\right\rangle$ & $\mathrm{eV}$ & & 7.379 & 8.621 & & $10.5 \pm 2.1$ & $9.97^{* *}$ \\
\hline$S_{1}$ & $\times 10^{-4}$ & & 3.95 & 4.263 & $3.8 \pm 0.6$ & $1.31 \pm 0.29$ & 3.0 \\
\hline$\left\langle\Gamma_{\gamma 1}\right\rangle$ & $\mathrm{meV}$ & & 132.2 & 130 & & & 133 \\
\hline$\left\langle\mathrm{D}_{2}\right\rangle$ & $\mathrm{eV}$ & & 4.664 & 5.173 & & & \\
\hline$S_{2}$ & $\times 10^{-4}$ & & 2.5 & 0.53 & & & \\
\hline$\left\langle\Gamma_{\gamma 2}\right\rangle$ & $\mathrm{meV}$ & & 132.2 & 130 & & & \\
\hline
\end{tabular}

* Average parameters at the low energy $(7.01 \mathrm{keV})$ of the unresolved region.

** Value at the neutron separation energy of ${ }^{110} \mathrm{Ag}$. 


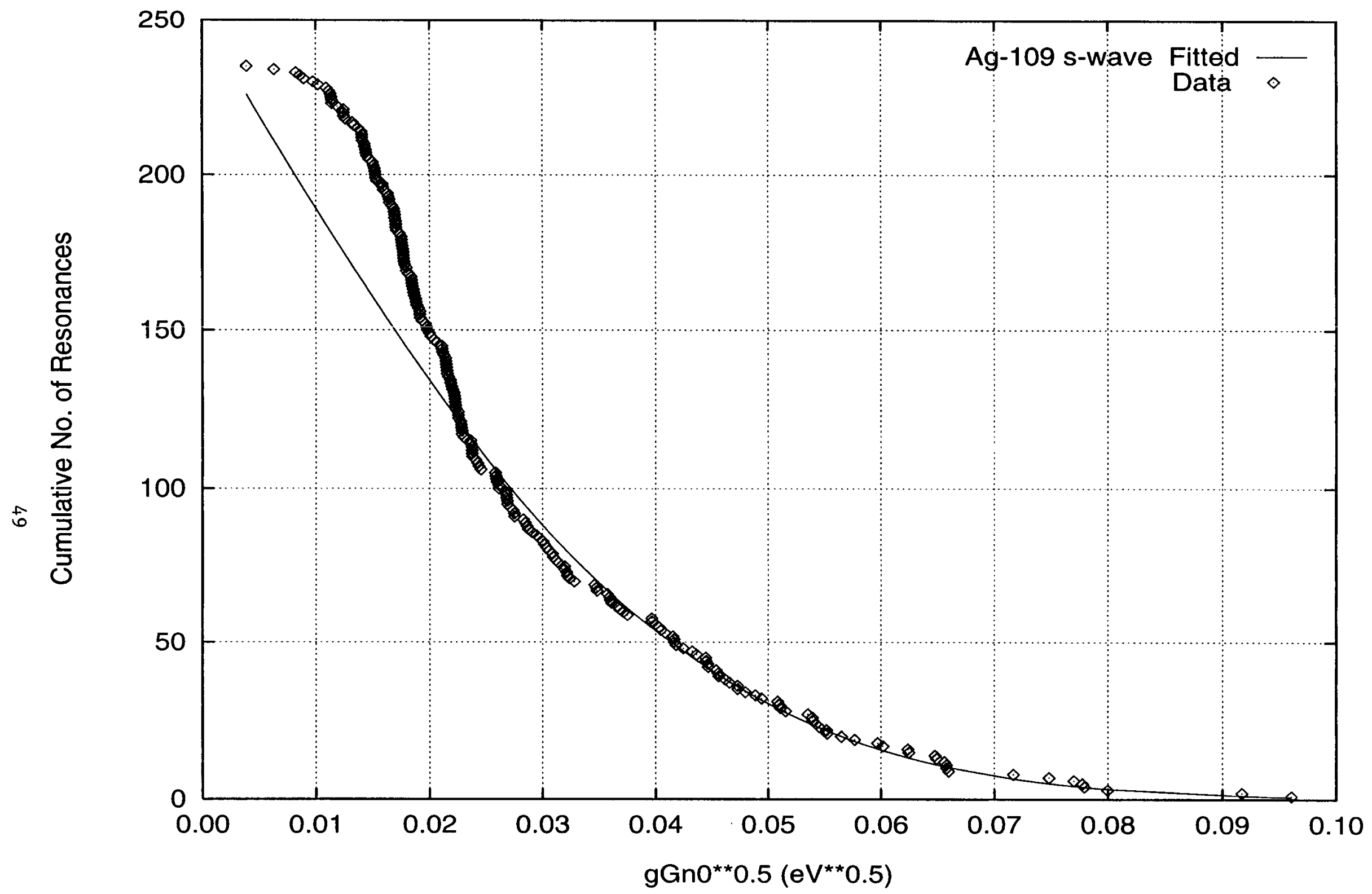

Fig. 20. Complement of the Cumulative Distribution of Neutron Reduced Widths ( ${ }^{109} \mathrm{Ag}$, s-wave) 


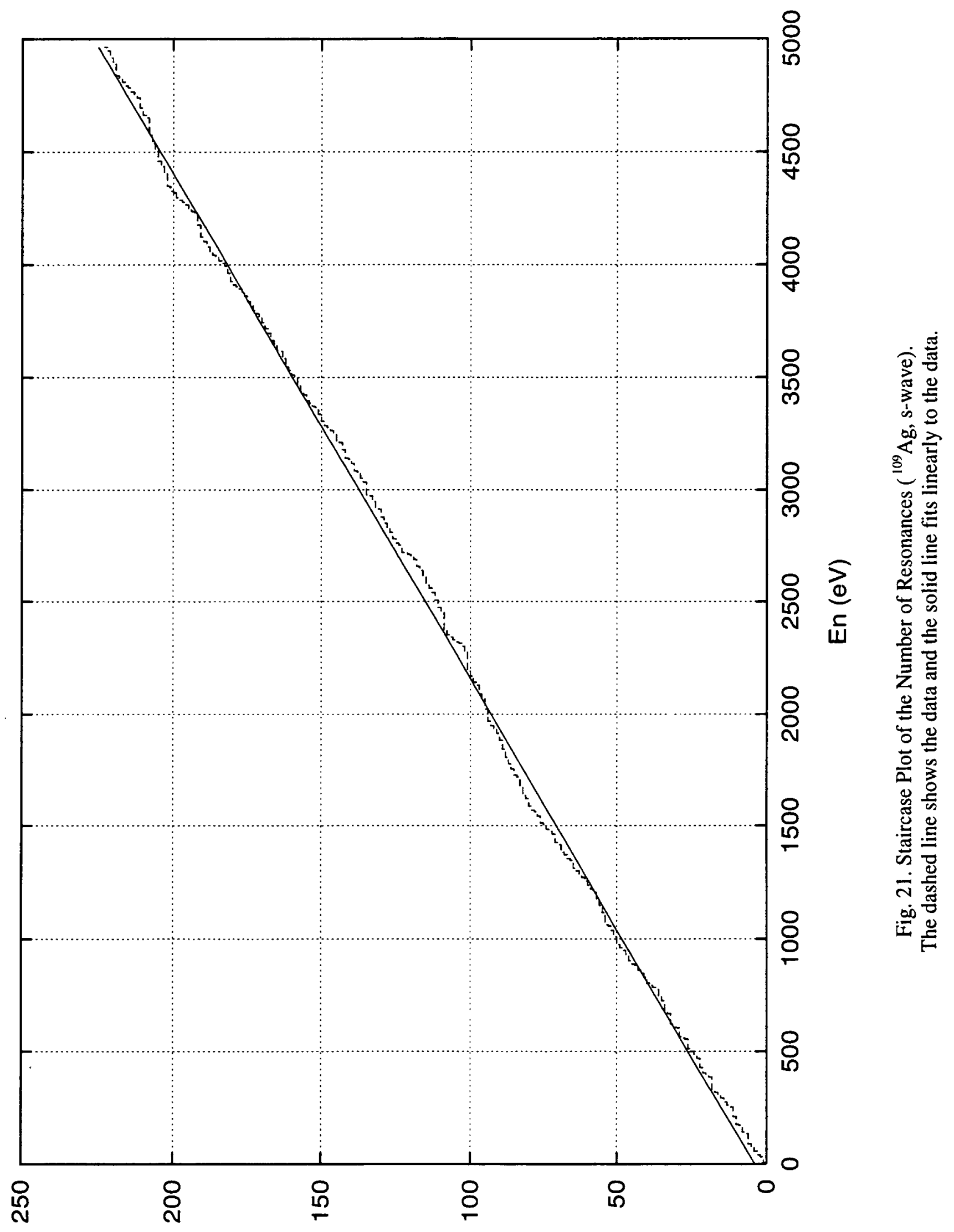

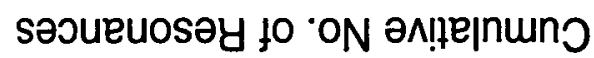




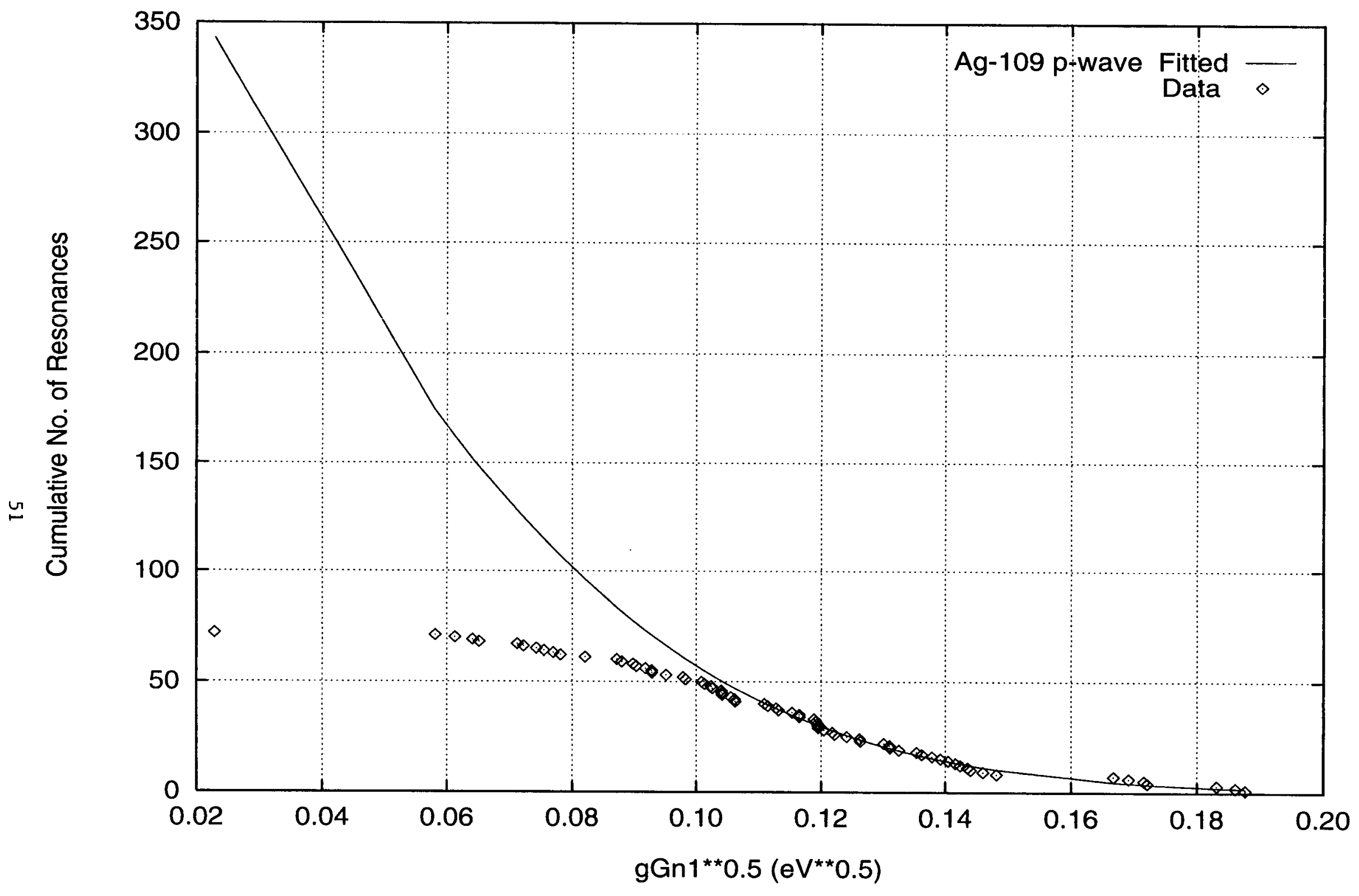

Fig. 22. Complement of the Cumulative Distribution of Neutron Reduced Widths ( ${ }^{109} \mathrm{Ag}$, p-wave) 


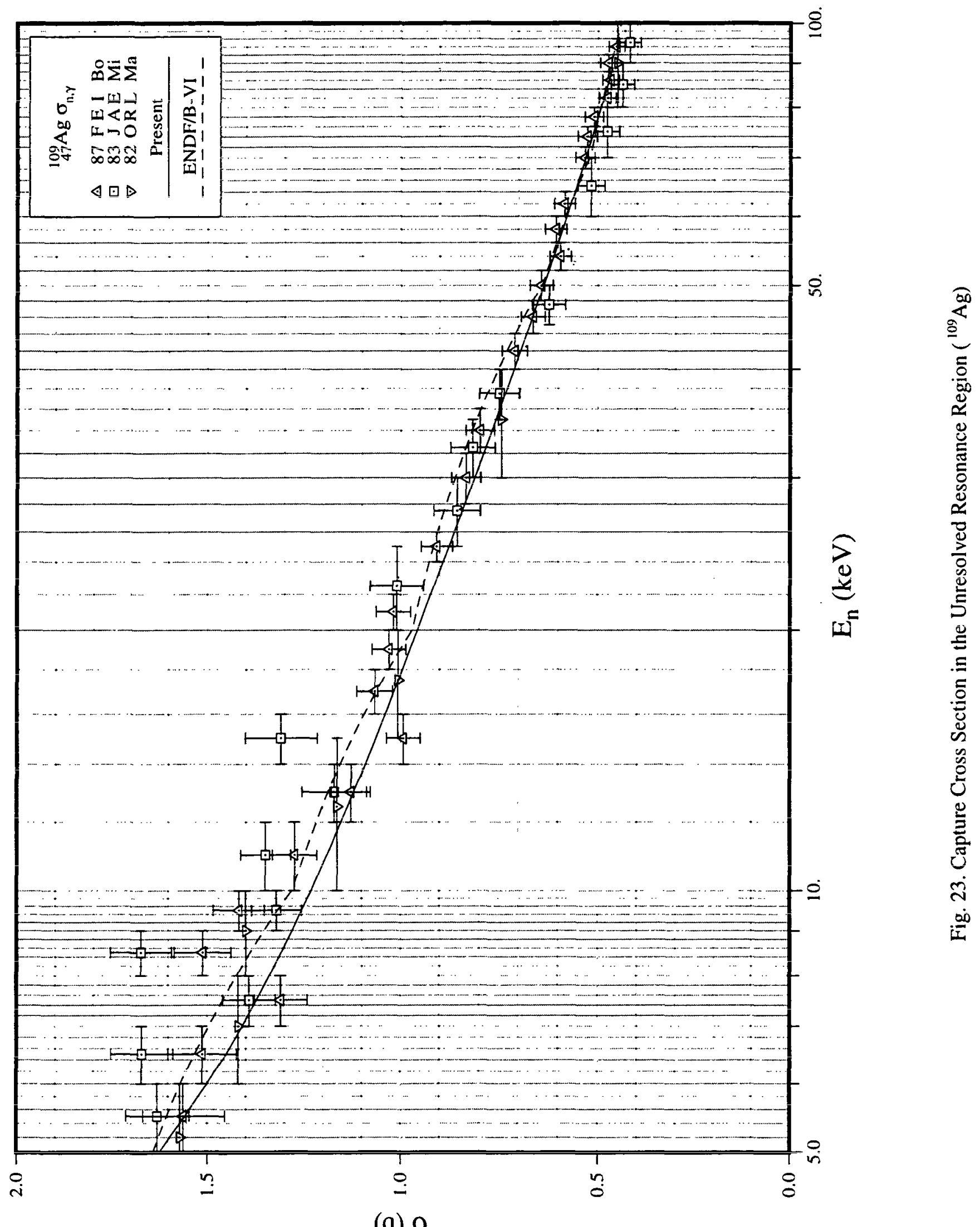


III.G. ${ }^{131} \mathrm{Xe}$

1. Thermal Region

To reproduce a thermal capture cross section of $90 \mathrm{~b}$, a bound level was stipulated. This capture cross-section was obtained by subtracting the isotopic contributions (mainly ${ }^{129} \mathrm{Xe}$ and ${ }^{131} \mathrm{Xe}$ ) from the natural element thermal capture cross section of $23.9 \pm 1.2$ b [Mu81]. An effective potential scattering radius, $R^{\prime}=5.4 \mathrm{fm}$, was adopted from a deformed optical model calculation reported in the BNL compilation [Mu81, Fig. 1]. The resonance parameters of the bound level were determined as: $\mathrm{E}_{0}=-26.65 \mathrm{eV}, \mathrm{J}=2, \Gamma_{\mathrm{n}}=303.5 \mathrm{meV}$, and $\Gamma_{\gamma}=112 \mathrm{meV}$. The contribution of a bound level to the thermal capture cross section was calculated as $22.1 \mathrm{~b}$. A comparison between the present thermal cross sections, as well as resonance capture and total integrals, with those of ENDF/B-VI, JEF-2 and JENDL-3 is presented in Table 16. While there is general agreement among the various evaluated thermal capture cross sections, discrepancies in the evaluated scattering cross sections exist. The capture resonance integral of the ENDF/BVI evaluation deviates from those of the present evaluation, JEF-2.2, JENDL-3.2, and the BNL compilation [Mu81].

\section{Resolved Resonance Parameters}

The resolved resonance parameters were adopted from the measurement of Ribon [Ri69]. On the basis of reported $\Gamma$ and $2 a g \Gamma_{\mathrm{n}}$, where $a$ is the abundance of Xe isotopes, five resonances at energies of $339.0,703.5,1380.3,1891.0$, and $2234.3 \mathrm{eV}$ were assigned to ${ }^{131} \mathrm{Xe}$ in the present study, in addition to the 41 resonances identified by Ribon for ${ }^{131} \mathrm{Xe}$. A p-wave resonance at 3.2 $\mathrm{eV}$ [Sk96] was included in the present evaluation. The present resolved energy region extends to $3.95 \mathrm{keV}$. Of a total of 47 positive-energy resonances, 45 were assigned as s-wave and 2 as pwave resonances by Bayasian analysis. Spins were assigned randomly to resonances for which the spin is unknown. This random assignment assumes that the level density is proportional to $(2 \mathrm{~J}+1)$. The uncertainty-iveighted averaging of 10 determined radiative widths resulted in an average value of $112 \pm 11 \mathrm{meV}$. This average value was assumed for those resonances for which radiative widths are unknown. The present average value is consistent with that reported in RIPL [Re98] (114 $\pm 37 \mathrm{meV})$, as well as that which is obtained from the s-wave systematics of radiative widths in the BNL compilation [Mu84, Fig. 6] around this mass region.

The distribution of reduced neutron widths for s-wave resonances was analyzed by fitting 36 resonances below $2 \mathrm{keV}$ to the Porter-Thomas distribution. As shown in Fig. 24, for a cutoff value of $0.4 \mathrm{meV}$ for the reduced width, the fit resulted in $\left\langle\mathrm{g} \Gamma_{\mathrm{n}}{ }^{0}\right\rangle=3.37 \pm 0.85 \mathrm{meV}$ and $\left\langle\mathrm{D}_{0}\right\rangle=42.8 \pm 5.0 \mathrm{eV}$. These values yield s-wave strength function of $\mathrm{S}_{0}=0.79 \pm 0.20$. The present $\left\langle\mathrm{D}_{0}\right\rangle$ is smaller than that presented in the BNL compilation, but is consistent with (45 $\left.\pm 15 \mathrm{eV}\right)$ [Re98]. While the present $S_{0}$ value deviates from the values (1.2 \pm 0.4$)$ [Mu81] and $(1.2 \pm 0.3)$ [Re98], it is consistent with Ribon's value of $0.85_{-0.17}^{+0.19}$ [Ri69]. 


\section{Unresolved Resonance Parameters}

The present unresolved resonance region covers the energy range from $3.95 \mathrm{keV}$ to 80.8 $\mathrm{keV}$. The latter energy corresponds to the threshold energy for neutron inelastic scattering to the first excited level of ${ }^{131} \mathrm{Xe}$. For this nucleus, s-, $\mathrm{p}$ - and d-wave average resonance parameters were provided. The level spacing varies with energy according to Gilbert-Cameron level density formula with associated parameters adopted from Mughabghab and Dunford [Mu98a] (neutron separation energy $=8.936 \mathrm{MeV}$, pairing energy $=2.16 \mathrm{MeV}$, and level density parameter $=10.58$ $\left.\mathrm{MeV}^{-1}\right)$. The dependence of the level spacing on $\exp \left\{-(\mathrm{J}+1 / 2)^{2} / 2 \sigma^{2}\right\}$, as well as $(2 \mathrm{~J}+1)$, was taken into account. With a value of $\sigma=3.0[\mathrm{Mu} 98 \mathrm{~b}]$ for the spin dispersion parameter, the level spacings for $p$ - and d-wave resonances are calculated as $1 / 1.73$ and $1 / 2.21$ of the s-wave spacing, respectively.

Unfortunately, capture cross section measurements were unavailable in the unresolved resonance region. The present unresolved resonance parameters are listed in the last column of Table 17. The s-wave average parameters are based on the analysis carried out in the resolved energy region. The value of $S_{1}$ is obtained from a deformed optical model calculation [Mu84, Fig. 4], and the value of $S_{2}$ was assumed to be same as that of $S_{0}$. An average radiative width of $112 \mathrm{meV}$ was assumed for $\mathrm{p}$ - and d-wave resonances. The capture cross section, constructed from the present average resonance parameters, is shown in Fig. 25 and is compared with other evaluations. The present cross section is consistent with the ENDF/B-VI evaluation.

The average capture cross section over a Maxwellian spectrum at $30 \mathrm{keV}$ was calculated as $306 \mathrm{mb}$. This value is apparently lower than that recommended in Beer's compilation, $453 \pm 81 \mathrm{mb}$ [Be92]. However, since Beer's value, as well as those reported in Bao's compilation [Ba87], is not based on measurements, the validity of the present evaluation is to be resolved by future measurements.

Table 16. Thermal Characteristics $\left({ }^{131} \mathrm{Xe}\right)$

\begin{tabular}{|c|c|c|c|c|c|c|c|}
\hline Quantity & Unit & $\begin{array}{c}\text { BNL } \\
{[\text { Mu81] }}\end{array}$ & $\begin{array}{c}\text { 98CRC } \\
{[\text { Ho98] }}\end{array}$ & $\begin{array}{c}\text { ENDF/ } \\
\text { B-VI }\end{array}$ & JEF-2.2 & $\begin{array}{c}\text { JENDL- } \\
3.2\end{array}$ & Present \\
\hline $\mathrm{R}^{\prime}$ & fm & & & 5.85 & 5.40 & 5.31 & 5.40 \\
\hline$\sigma_{\gamma}^{0}$ & barn & $85 \pm 10$ & $90 \pm 10$ & 90.6 & 85.1 & 85.0 & 90.0 \\
$\sigma_{\mathrm{s}}{ }^{0}$ & barn & & & 4.32 & 24.3 & 24.0 & 1.19 \\
\hline $\mathrm{g}_{\mathrm{w}}{ }^{*}$ & & & & 0.9996 & 1.001 & 1.001 & 1.0014 \\
\hline RI-capt. & barn & $900 \pm 100$ & $900 \pm 100$ & 1016 & 890 & 900 & 882 \\
RI-total & barn & & & 2877 & 2958 & 2938 & 2765 \\
\hline
\end{tabular}

* Westcott factor for capture cross section. 
Table 17. Average Resonance Parameters for the Unresolved Resonance Region $\left({ }^{131} \mathrm{Xe}\right)$

\begin{tabular}{|c|c|c|c|c|c|c|c|}
\hline \multirow[b]{2}{*}{ Quantity } & \multirow[b]{2}{*}{ Unit } & \multirow{2}{*}{$\begin{array}{c}\text { B.NL } \\
\text { [Mu81] }\end{array}$} & \multirow{2}{*}{$\begin{array}{c}\text { ENDF/ } \\
\text { B-VI }\end{array}$} & \multirow[b]{2}{*}{ JEF-2.2 } & \multirow{2}{*}{$\begin{array}{c}\text { JENDL- } \\
3.2^{*}\end{array}$} & \multicolumn{2}{|c|}{ Present } \\
\hline & & & & & & $\begin{array}{c}\text { PT } \\
\text { Analysis }\end{array}$ & Adopted \\
\hline $\mathbf{R}^{\prime}$ & $\mathrm{fm}$ & & & & 5.63 & & 5.40 \\
\hline $\begin{array}{c}\left\langle\mathrm{D}_{0}\right\rangle \\
\mathrm{S}_{\mathbf{0}} \\
\left\langle\Gamma_{\gamma_{0}}\right\rangle\end{array}$ & $\begin{array}{c}\mathrm{eV} \\
\times 10^{-4} \\
\mathrm{meV}\end{array}$ & $\begin{array}{c}70 \pm 20 \\
1.2 \pm 0.4\end{array}$ & & & $\begin{array}{c}35.5 \\
0.70 \\
114\end{array}$ & $\begin{array}{c}42.8 \pm 5.0 \\
0.79 \pm 0.20\end{array}$ & $\begin{array}{c}42.8^{* *} \\
0.79 \\
112.3\end{array}$ \\
\hline $\begin{array}{c}\left\langle\mathrm{D}_{1}\right\rangle \\
\mathrm{S}_{1} \\
\left\langle\Gamma_{\gamma 1}\right\rangle\end{array}$ & $\begin{array}{c}\mathrm{eV} \\
\times 10^{-4} \\
\mathrm{meV} \\
\end{array}$ & & & & $\begin{array}{c}17.7 \\
1.58 \\
114\end{array}$ & & $\begin{array}{c}24.7^{* *} \\
1.40 \\
112.3\end{array}$ \\
\hline $\begin{array}{c}\left\langle\mathbf{D}_{2}\right\rangle \\
S_{2} \\
\left\langle\Gamma_{\gamma_{2}}\right\rangle \\
\end{array}$ & $\begin{array}{c}\mathrm{eV} \\
\times 10^{-4} \\
\mathrm{meV} \\
\end{array}$ & & & & $\begin{array}{c}11.3 \\
0.99 \\
114\end{array}$ & & $\begin{array}{c}19.4^{* *} \\
0.79 \\
112.3\end{array}$ \\
\hline
\end{tabular}

* Average parameters at the low energy bound of the unresolved resonance region $(2.25 \mathrm{keV})$. ** Value at the neutron separation energy of ${ }^{132} \mathrm{Xe}$. 


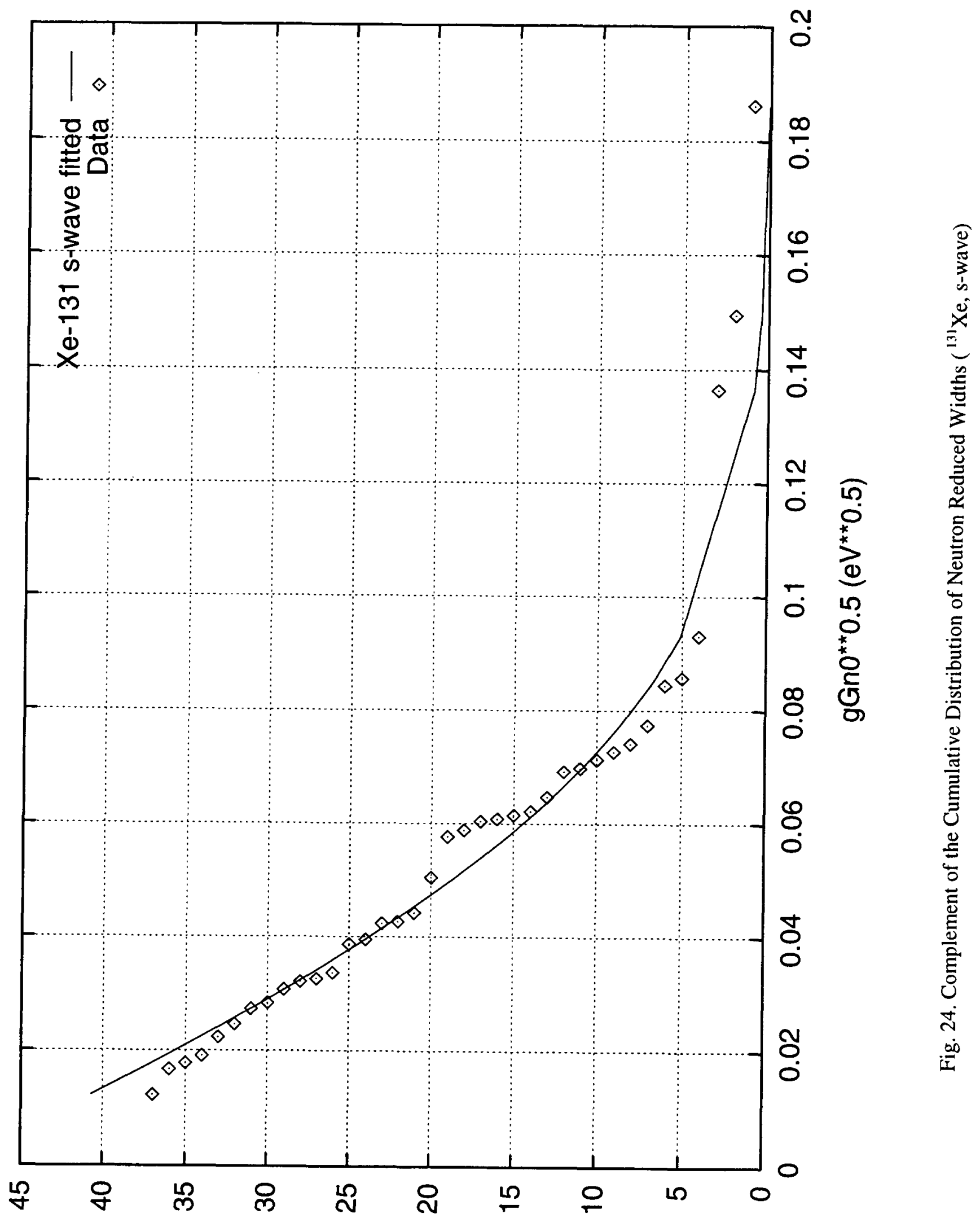

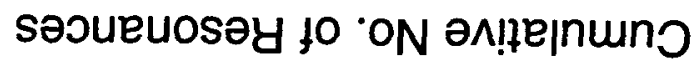




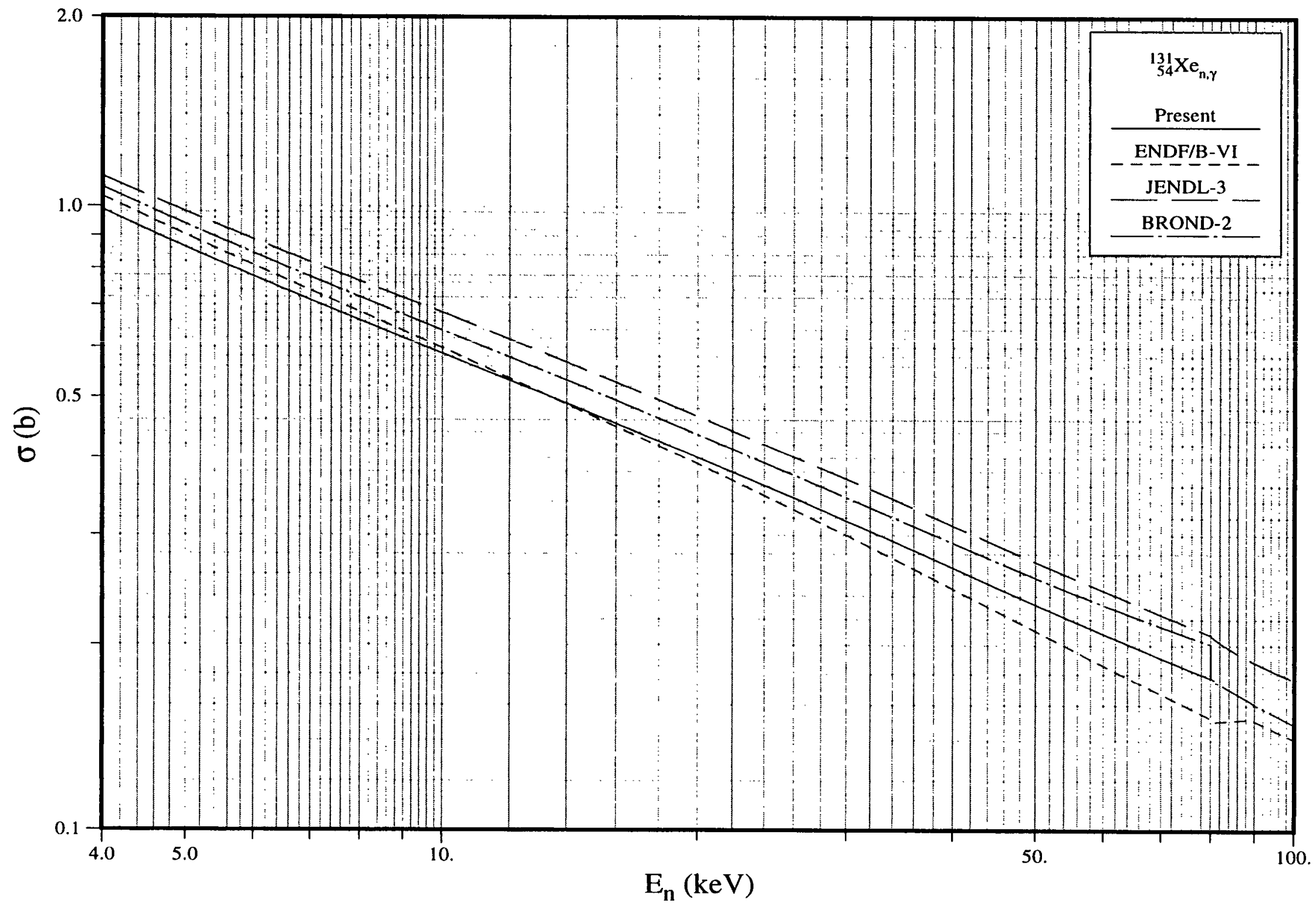

Fig. 25. Capture Cross Section in the Unresolved Resonance Region $\left({ }^{131} \mathrm{Xe}\right)$ 


\section{II.H. ${ }^{133} \mathrm{Cs}$}

\section{Thermal Region}

In the present evaluation, two bound levels were invoked to reproduce a thermal capture cross section, $\sigma_{\gamma}{ }^{0}=29.0 \pm 1.5 \mathrm{~b}$, recommended in Mughabghab's BNL compilation [Mu81], an incoherent scattering length, $b_{+}-b_{-}=2.61 \pm 0.3 \mathrm{fm}$, measured by Glaettli et al. [G179], and a bound coherent scattering length, $b=5.42 \pm 0.02 \mathrm{fm}$, adopted from Koester's compilation [Ko91]. An effective scattering radius, $R^{\prime}=5.3 \mathrm{fm}$ was obtained from the BNL compilation [Mu81]. In addition, the thermal capture contribution of the bound level with spin 4 was assumed as 3 times that of the bound level with spin 3. The contribution of positive energy resonances to the thermal capture cross section was calculated as $19.5 \mathrm{~b}$. The bound level resonance parameters are listed in Table 18. A comparison of the present thermal cross sections and resonance integrals with those of ENDF/B-VI.5, JEF-2.2 and JENDL-3.2 evaluations is presented in Table 19. The thermal capture cross sections are in very good agreement with each other. The capture resonance integral of the present evaluation is in good agreement with those recommended in the BNL compilation [Mu81] and Holden compilation [Ho98].

\section{Resolved Resonance Parameters}

The primary source of resolved resonance parameters for this nuclide is the BNL compilation [Mu81]. In addition to this source, measurements by Nakajima et al. [Na90], Macklin [Ma82c], and Popov and Tzeciak [Po81] were incorporated into the present set of parameters. By combining the capture data with the transmission data, radiative widths of some resonances were derived in favorable cases. The resolved energy region extends up to $3.99 \mathrm{keV}$. Resonances above $3.99 \mathrm{keV}$ [Na90, Ma82] were represented with average resonance parameters in the unresolved resonance region. On the basis of Bayesian analysis, 148 and 39 resonances were assigned as s-wave and p-wave resonances, respectively. Resonance spins were assigned randomly to those resonances with unknown spins. The random assignment assumes the level density is proportional to $(2 \mathrm{~J}+1)$. The uncertainty-weighted averaging of 31 measured radiative widths resulted in an average value of $123 \pm 4 \mathrm{meV}$. This value was assumed for those resonances for which the radiative widths are unknown. The present average value is consistent with values presented in the BNL compilation (120 meV) [Mu81], RIPL [Re98] (120 $\pm 10 \mathrm{meV})$,

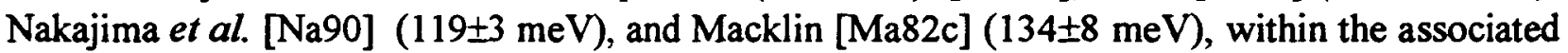
uncertainty range.

The distributions of s- and p-wave reduced neutron widths were analyzed in terms of the Porter-Thomas distribution. For s-wave resonances, those with reduced widths smaller than $\mathbf{0 . 2}$ $\mathrm{meV}$ were excluded in the analysis. The fit for the s-wave resonances, Fig. 26, resulted in $\left\langle\mathrm{g} \Gamma_{\mathbf{n}}{ }^{0}\right\rangle=1.44 \pm 0.17 \mathrm{meV}$ and $\left\langle\mathrm{D}_{0}\right\rangle=19.8 \pm 0.9 \mathrm{eV}$. These values yield an s-wave strength function of $S_{0}=0.73 \pm 0.09$. The present $\left\langle D_{0}\right\rangle$ is consistent with values recommended in the BNL compilation $(20.65 \pm 2.3 \mathrm{eV})$ [Mu81] and RIPL [Re98] $(21 \pm 2 \mathrm{eV})$. However, it deviates from the values reported by Nakajima et al. [Na90], $22.4 \pm 1.5 \mathrm{eV}$, and Anufriev et al. [An78], 16.3 \pm 2.3 $\mathrm{eV}$. The present $\mathrm{S}_{0}$ is consistent again with values in the BNL compilation, $0.70 \pm 0.07$, and RIPL, $0.76 \pm 0.10$, as well as with that reported by Nakajima et al. [Na90] $0.77 \pm 0.09$. For p-wave resonances, the $\chi^{2}$ value of the fit for all the observed resonances was very large. It is clear from 
Fig. 27 that many weak p-wave resonances were missed. Subsequently, resonances with reduced widths, $\left\langle\mathrm{g} \Gamma_{\mathrm{a}}{ }^{1}\right\rangle$, smaller than $12.5 \mathrm{meV}$ were excluded from the analysis. As shown in Fig. 27, we obtained a good fit, with a $95 \%$ confidence limit, which resulted in $\left\langle\mathrm{g} \Gamma_{\mathrm{n}}{ }^{1}\right\rangle=5.27 \pm 1.25 \mathrm{meV}$, $\left\langle D_{1}\right\rangle=14.0 \pm 3.5 \mathrm{eV}$ and $S_{1}=1.25 \pm 0.37$. Because of the small sample size for the p-wave population, the uncertainties of the derived average quantities are large.

\section{Unresolved Resonance Parameters}

The present unresolved resonance region covers the energy range from $3.99 \mathrm{keV}$ to 81.6 $\mathrm{keV}$. The latter energy corresponds to the threshold energy of neutron inelastic scattering to the first excited level of ${ }^{133} \mathrm{Cs}$. For this nucleus, s-, p- and d-wave average resonance parameters were provided. The level spacing varies with energy according to Gilbert-Cameron level density formula with associated parameters adopted from Mughabghab and Dunford [Mu98a] (neutron separation energy $=6.891 \mathrm{MeV}$, pairing energy $=0.0 \mathrm{MeV}$, and level density parameter $=14.2$ $\mathrm{MeV}^{-1}$ ). Because of the large target spin of this nuclide, $7 / 2$, the dependence of level spacing on $(2 \mathrm{~J}+1) \exp \left\{-(\mathrm{J}+1 / 2)^{2} / 2 \sigma^{2}\right\}$ was taken into account. With a value of $\sigma=2.8$ for the spin dispersion parameter [Mu98b], the level spacing for $\mathrm{p}$ - and d-wave resonances were calculated as $1 / 1.87$ and $1 / 2.49$ of the s-wave spacing, respectively. These values deviate from $1 / 2$ and $1 / 3$, obtained by assuming only a $(2 \mathrm{~J}+1)$ dependence of the level spacing.

In the unresolved resonance region, the average capture cross sections measured by Bokhovko et al. [Bo91], Yamamuro et al. [Ya83], and Macklin [Ma82c] were taken into consideration. These measurements agree with each other, while earlier data, included in McLane's compilation [Mc88], deviate from the above measurements. As shown in Fig. 28, the present unresolved resonance parameters resulted in a good fit to the experimental data. The present parameters are listed in the last column of Table 20. The values of $\left\langle\Gamma_{\gamma}\right\rangle,\left\langle D_{0}\right\rangle$ and $S_{0}$ are those which were obtained from the analysis of resolved energy region. The present value of $S_{1}$ was adopted from a deformed optical model calculation [Mu84, Fig. 4]; the value of $S_{2}$ was assumed to be the same as that of $S_{0}$. It is noted that the $p$ - and d-wave strength functions are consistent with those derived by Bokhovko et al. $[B 091]\left(S_{1}=1.3 \pm 0.4\right.$ and $\left.S_{2}=0.7 \pm 0.2\right)$. The average capture cross section for a Maxwellian spectrum at $30 \mathrm{keV}$ is calculated as $513 \mathrm{mb}$. For comparisons with other calculations, see Table 56.

Table 18. Bound Level Resonance Parameters $\left({ }^{133} \mathrm{Cs}\right)$

\begin{tabular}{|c|c|c|c|}
\hline Energy (eV) & $l$ and Resonance Spin & Neutron Width (meV) & $\begin{array}{c}\text { Capture Width } \\
(\mathrm{meV})\end{array}$ \\
\hline-41.32 & $L=0, \mathrm{~J}=4.0$ & 273.6 & 123.0 \\
-25.21 & $l=0, \mathrm{~J}=3.0$ & 34.1 & 123.0 \\
\hline
\end{tabular}


Table 19. Thermal Characteristics $\left({ }^{133} \mathrm{Cs}\right)$

\begin{tabular}{|c|c|c|c|c|c|c|c|}
\hline Quantity & Unit & $\begin{array}{c}\text { BNL } \\
{[\text { Mu81] }}\end{array}$ & $\begin{array}{c}\text { 98CRC } \\
\text { [Ho98] }\end{array}$ & $\begin{array}{c}\text { ENDF/ } \\
\text { B-VI }\end{array}$ & JEF-2.2 & $\begin{array}{c}\text { JENDL- } \\
3.2\end{array}$ & Present \\
\hline $\mathrm{R}^{\prime}$ & fm & $5.3 \pm 0.5$ & & 7.517 & 5.30 & 5.35 & 5.30 \\
\hline$\sigma_{\gamma}{ }^{0}$ & barn & $29.0 \pm 1.5$ & 30.4 & 29.7 & 29.1 & 29.0 & 29.0 \\
$\sigma_{\mathrm{s}}{ }^{0}$ & barn & & & 4.98 & 3.88 & 4.29 & 3.97 \\
\hline $\mathrm{g}_{w}{ }^{*}$ & & & & 1.004 & 1.002 & 1.002 & 1.0029 \\
\hline RI-capt. & barn & $437 \pm 26$ & 422 & 383 & 439 & 396 & 421 \\
RI-total & barn & & & 518 & 556 & 508 & 535 \\
\hline
\end{tabular}

*Westcott factor for capture cross section.

Table 20. Average Resonance Parameters for Unresolved Resonance Region $\left({ }^{133} \mathrm{Cs}\right)$

\begin{tabular}{|c|c|c|c|c|c|c|c|}
\hline \multirow[b]{2}{*}{ Quantity } & \multirow[b]{2}{*}{ Unit } & \multirow[b]{2}{*}{$\begin{array}{c}\text { BNL } \\
\text { [Mu81] }\end{array}$} & \multirow[b]{2}{*}{$\begin{array}{c}\text { ENDF/ } \\
\text { B-VI }\end{array}$} & \multirow[b]{2}{*}{ JEF-2.2 } & \multirow[b]{2}{*}{$\begin{array}{l}\text { JENDL- } \\
3.2^{*}\end{array}$} & \multicolumn{2}{|c|}{ Present } \\
\hline & & & & & & $\begin{array}{c}\text { PT } \\
\text { Analysis }\end{array}$ & Adopted \\
\hline $\mathbf{R}^{\prime}$ & $\mathrm{fm}$ & $5.3 \pm 0.5$ & & 5.50 & 5.84 & & 5.30 \\
\hline$\overline{\left\langle\mathrm{D}_{0}\right\rangle}$ & $\mathrm{eV}$ & $20.65 \pm 2.3$ & & 21.8 & 16.53 & $19.8 \pm 0.9$ & $19.8^{* *}$ \\
\hline$S_{0}$ & $\times 10^{-4}$ & $0.7 \pm 0.07$ & & 0.8 & 0.70 & $0.73 \pm 0.09$ & 0.73 \\
\hline$\left\langle\Gamma_{\gamma 0}\right\rangle$ & $\mathrm{meV}$ & 120 & & 125.3 & 120.0 & & 123.0 \\
\hline$\left\langle\mathrm{D}_{1}\right\rangle$ & $\mathrm{eV}$ & & & 11.25 & 8.27 & $14.0 \pm 3.5$ & $10.6^{2 *}$ \\
\hline$S_{1}$ & $\times 10^{-4}$ & & & 4.0 & 1.40 & $1.25 \pm 0.37$ & 1.40 \\
\hline$\left\langle\Gamma_{\gamma_{1}}\right\rangle$ & $\mathrm{meV}$ & & & 125.3 & 120.0 & & 123.0 \\
\hline$\left\langle\mathrm{D}_{2}\right\rangle$ & $\mathrm{eV}$ & & & 7.91 & 5.51 & & $7.95^{* *}$ \\
\hline$S_{2}$ & $\times 10^{-4}$ & & & 1.64 & 1.30 & & 0.73 \\
\hline$\left\langle\Gamma_{\gamma 2}\right\rangle$ & $\mathrm{meV}$ & & & 125.3 & 120.0 & & 123.0 \\
\hline
\end{tabular}

* Average parameters at the low energy of the unresolved resonance region $(5.98 \mathrm{keV})$.

${ }^{* *}$ Value at the neutron separation energy of ${ }^{134} \mathrm{Cs}$. 


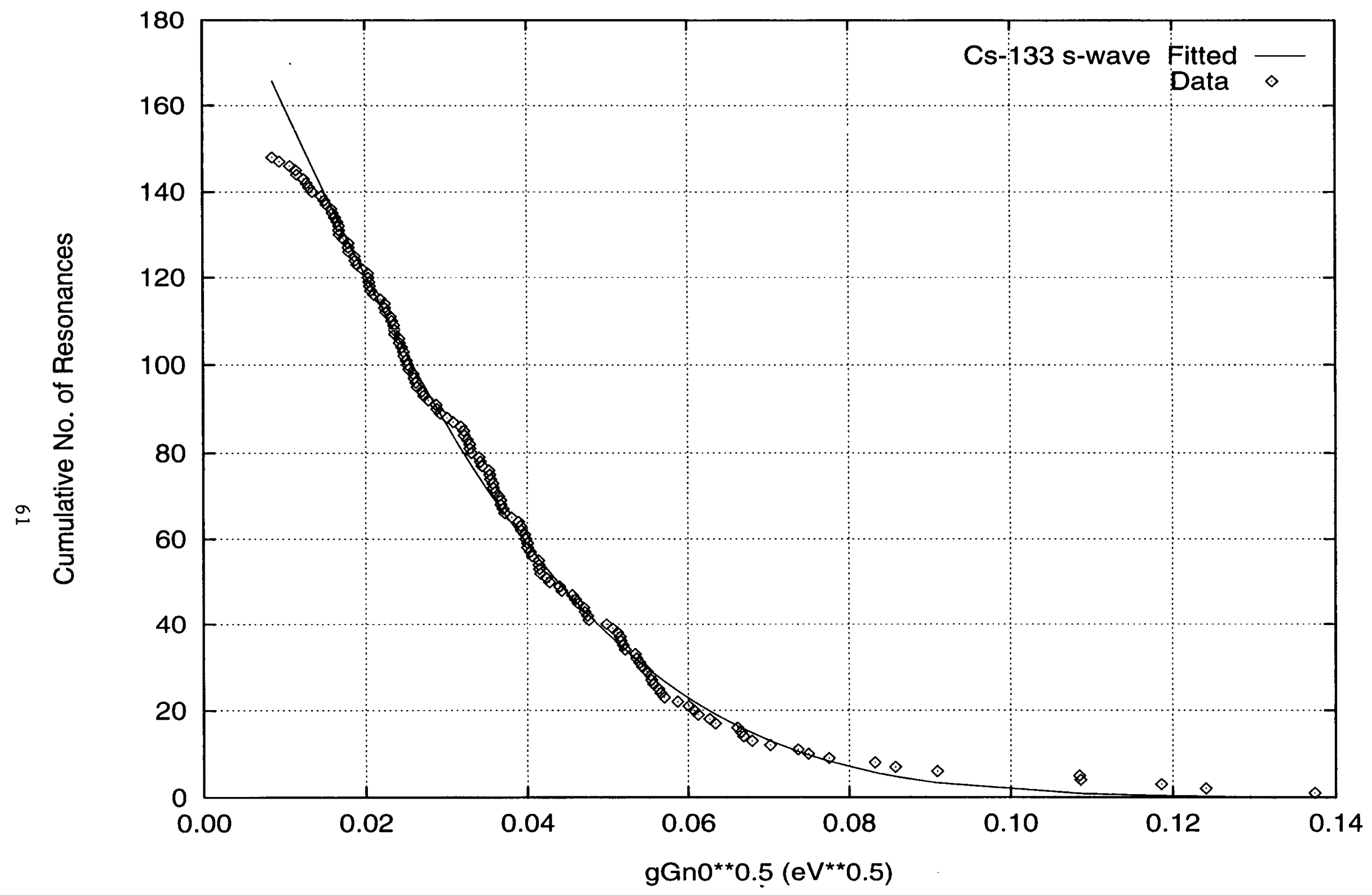

Fig. 26. Complement of the Cumulative Distribution of Neutron Reduced Widths ( ${ }^{133} \mathrm{Cs}$, s-wave) 


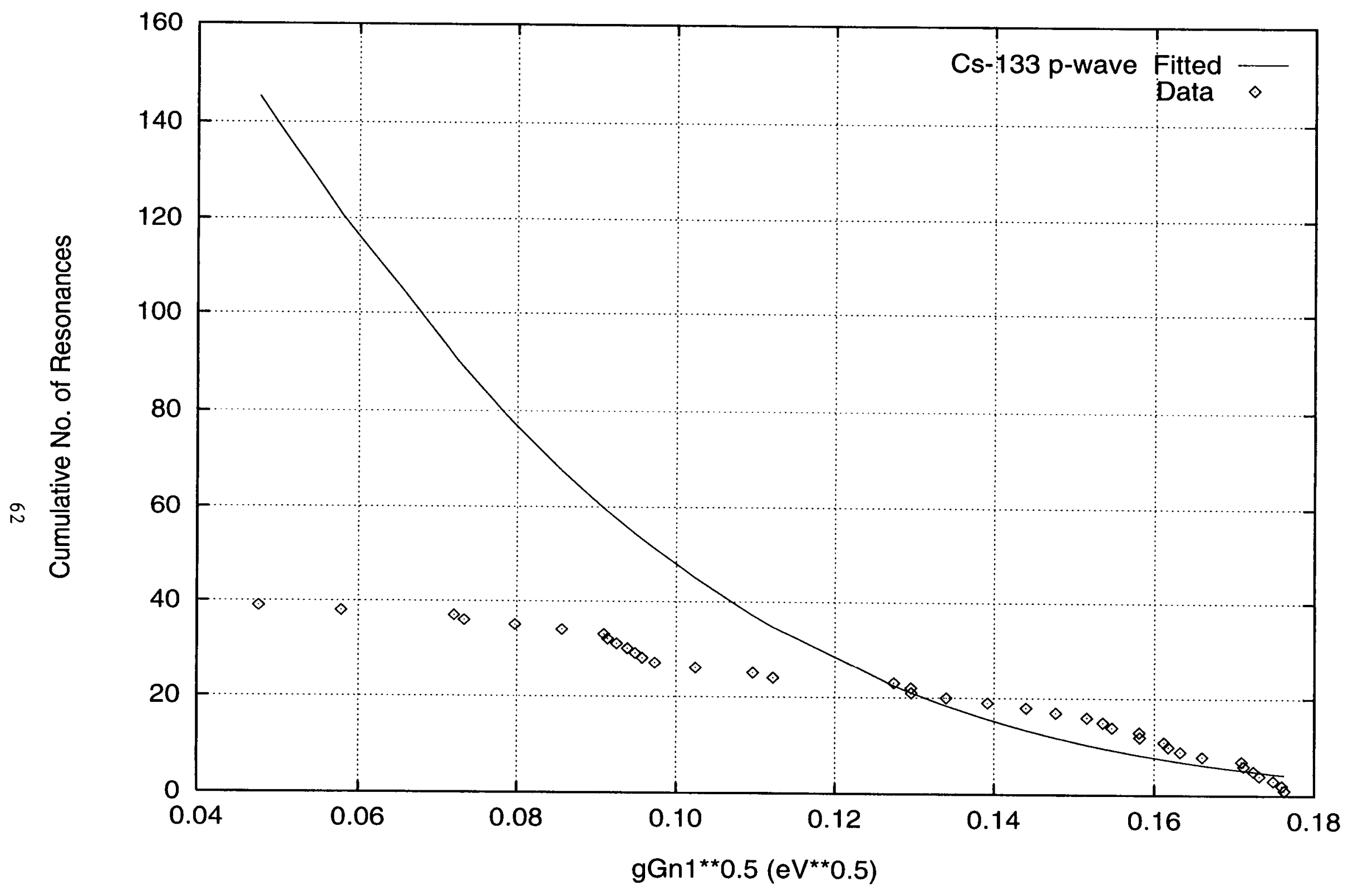

Fig. 27. Complement of the Cumulative Distribution of Neutron Reduced Widths ( ${ }^{133} \mathrm{Cs}$, $\mathrm{p}$-wave) 


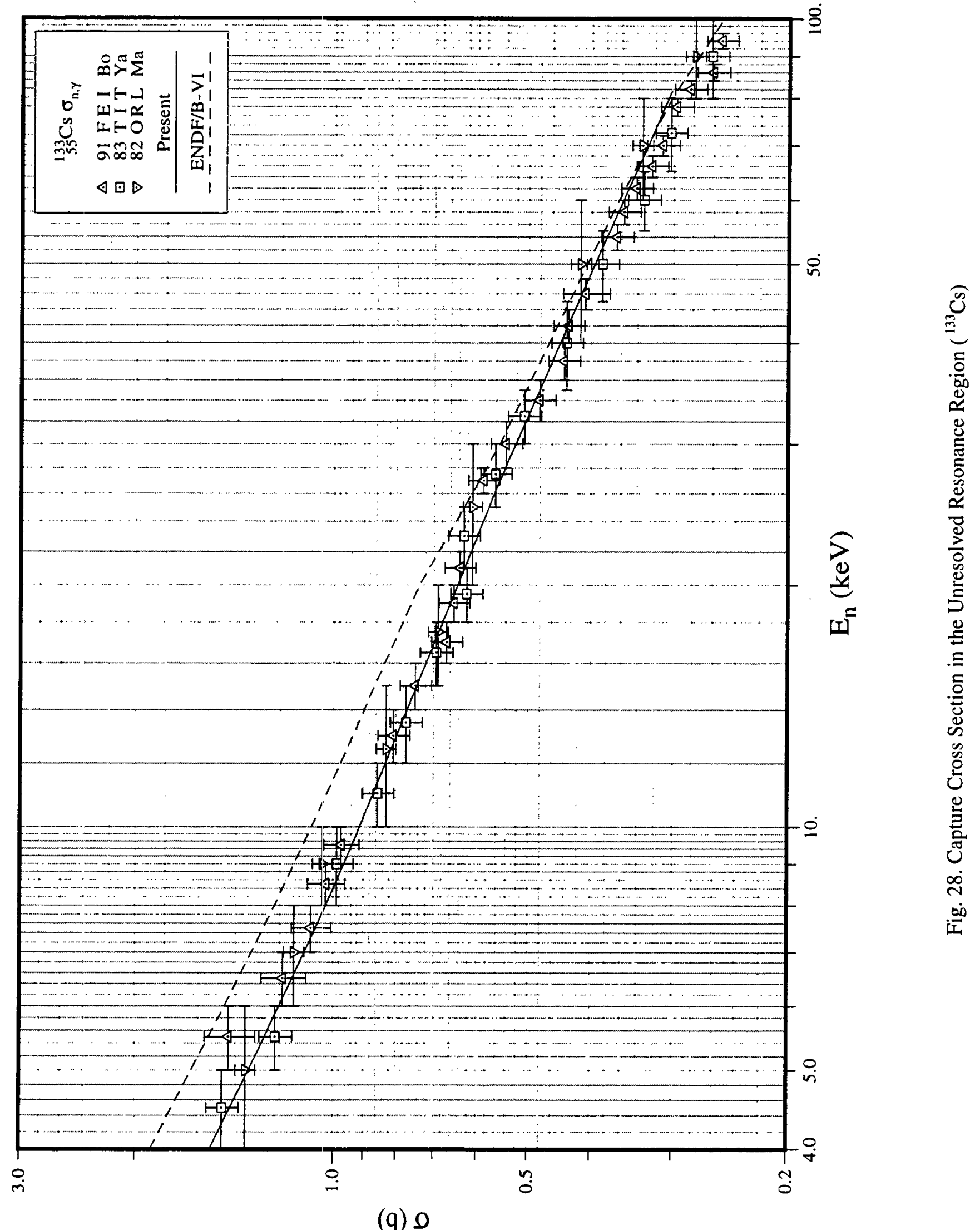




\section{III.I. ${ }^{141} \mathrm{Pr}$}

\section{Thermal Region}

Positive-energy resolved resonance parameters and an effective scattering radius $R$ ' of $4.93 \mathrm{fm}$ were adopted basically from Mughabghab's BNL compilation [Mu81]. However, two new bound levels were invoked to reproduce a thermal capture cross section of $11.5 \mathrm{~b}$ [Mu81], a bound coherent scattering length of $4.58 \pm 0.05 \mathrm{fm}$ [Ko91], and an incoherent scattering length $\left(b_{+}-b_{-}\right)$of $-7.2 \pm 0.07 \mathrm{fm}$ [Ak76, Ko91]. An attempt to fit the thermal capture and scattering cross sections with a single bound level resulted in an unreasonable value for the scattering radius $\left(R^{\prime}=7.2 \mathrm{fm}\right)$, which is at variance with a value of $4.9 \pm 0.5 \mathrm{fm}$ [Mu81]. On this basis, two bound levels were invoked. To determine the parameters of the two bound levels, we assumed that the capture cross section for the bound level with spin 3 is equal to the other bound with spin 4. The resulting parameters for the two bound levels are listed in Table 21.

As shown in Table 22, the various quantities computed in the present study are generally in good agreement with those in the JEF-2 and JENDL-3 evaluations. However, a disagreement between the present evaluation and that of ENDF/B-VI for the capture and total resonance integrals exists.

\section{Resolved Resonance Parameters}

Resonance parameters up to $10.049 \mathrm{keV}$ were adopted basically from the BNL compilation [Mu81]. In addition to these data, new measurements were taken into consideration. These are the capture and scattering widths of Taylor et al. [Ta79], the spin determinations of Alfimenkov et al. [Al82a], and the $l$ assignments of Morgenstern et al. [Mo69]. Uncertaintyweighted averaging of known 69 radiative widths resulted in a value of $86 \pm 2 \mathrm{meV}$, which was assumed for resonances with unknown radiative widths. Of a total of 126 resonances, 79 (61 previously assigned by measurements) were assigned as s-wave and 47 ( 6 previously assigned) as $p$-wave by Bayesian analysis. We remark that resonances, with relatively large neutron widths, which were determined as $\mathrm{p}$-wave resonances by the transmission measurements, were assigned as s-wave resonances by the Bayesian analysis. As an example, the p-wave resonance at $3395 \mathrm{eV}$ with $\mathrm{g} \Gamma_{\mathrm{n}}=80 \mathrm{meV}$ was determined by Bayesian analysis as s-wave with a probability of 0.98 . Spins of resonances with undetermined values were assigned randomly on the basis of $(2 \mathrm{~J}+1)$ law.

The distribution of reduced neutron widths was analyzed on the basis of the PorterThomas distribution. For s-wave resonances, Fig. 29 shows the calculated cumulative numbers of s-wave resonances with widths larger than a given $\operatorname{Vg}_{\mathrm{g}}{ }^{0}$ value. The fit resulted in $\left\langle\mathrm{D}_{0}\right\rangle=118.0 \pm 7.2 \mathrm{eV}$ and $\left\langle\mathrm{g}_{\mathrm{n}}{ }^{0}\right\rangle=20.9 \pm 3.4 \mathrm{meV}$. These values yield s-wave strength function of $S_{0}=1.77 \pm 0.3$. The present $\left\langle D_{0}\right\rangle$ is consistent with values reported by Taylor et al. [Ta79], $116 \pm 10 \mathrm{eV}$, and RIPL [Re98], $110 \pm 20$, but is larger than that in the BNL compilation, $88 \pm 9 \mathrm{eV}$ [Mu81]. The $S_{0}$ value is consistent with those reported in [Ta79], 1.8, RIPL, 1.7 \pm 0.3 , and the BNL compilation [Mu81], 1.5 \pm 0.2 . As shown in Fig. 30, the p-wave Porter-Thomas distribution without excluding weak resonances resulted in $\left\langle D_{1}\right\rangle=101.6 \pm 10.9 \mathrm{eV}$ and $S_{1}=0.65 \pm 0.14$. The $\mathrm{p}-$ wave level spacing is unreasonably large when compared with an expected value of about $60 \mathrm{eV}$. 
This indicated that several p-wave resonances were not detected experimentally. On the other hand, the p-wave strength function is consistent with the systematics study of p-wave strength functions in this mass region [Mu81].

\section{Unresolved Resonance Parameters}

The upper energy of the unresolved energy region was set to $146.47 \mathrm{keV}$, which corresponds to the threshold energy for neutron inelastic scattering to the first excited level of ${ }^{141} \mathrm{Pr}$ at $145.44 \mathrm{keV}$.

The capture cross section measurements of Taylor et al. [Ta79], subsequently corrected by Allen et al. [Al82b], Zaikin et al. [Za71], Konks et al. [Ko64], and Gibbons et al. [Gi61] were taken into consideration in this evaluation. Other measurements in the EXFOR database [EX], which deviated considerably from these measurements, were not considered.

In this energy region, average resonance parameters for $s-, p$ - and $d$-waves were provided. Since the upper energy of the unresolved energy region is relatively high, $d$-wave contribution is not negligible. An energy-dependent level spacing according to Gilbert-Cameron level density formula, with parameters adopted from Mughabghab and Dunford [Mu98a], was adopted. From the $(2 \mathrm{~J}+1)$ dependence of the level density, level spacings for $\mathrm{p}$ - and $\mathrm{d}$-wave resonances were calculated as $1 / 2$ and $1 / 3$ of the value for $s$-wave spacing, respectively. In the first attempt, the following average parameters were implemented in the computation of the capture cross section: 1) the average resonance parameters, $\left\langle D_{0}\right\rangle, S_{0}$ and $S_{1}$, derived from the resolved resonance region; 2) $S_{2}=3.0$, obtained from a spherical optical model calculation [Mu84]; and 3) $\left\langle\Gamma_{\gamma}\right\rangle=86 \mathrm{meV}$ for s-, p-, and d-wave resonances. The resulting calculation is in very good agreement with the measurements in the $10-40 \mathrm{keV}$ region [Ta79]. However, in the high energy region, there is a discrepancy of up to $30 \%$. To achieve an improved fit in the highenergy region, $S_{2}$ was decreased to 0.6 and $S_{1}$ was slightly increased to 0.75 . This small d-wave strength function is in reasonable agreement with measured strength function data in this mass region [Mu84]. Our finalized parameters for the unresolved region are listed in the last column of Table 23 and are compared with other evaluations. The calculated capture cross section with the adjusted parameters is shown in Fig. 31 and is compared with measurements [Ta79, Za71, Ko64, Gi63].

Maxwellian-averaged capture cross section for a temperature of $30 \mathrm{keV}$ was computed as $117 \mathrm{mb}$. This cross section is in excellent agreement with $119 \pm 15 \mathrm{mb}$ reported in Beer's compilation [Be92] and $111 \pm 15 \mathrm{mb}$ in the BNL compilation [Mu81].

Table 21. Bound Level Resonance Parameters $\left({ }^{141} \mathrm{Pr}\right)$

\begin{tabular}{|c|c|c|c|}
\hline Energy (eV) & $l$ and Resonance Spin & $\begin{array}{c}\text { Scattering Width } \\
(\mathrm{meV})\end{array}$ & $\begin{array}{c}\text { Capture Width } \\
(\mathrm{meV})\end{array}$ \\
\hline-48.12 & $l=0, \mathrm{~J}=3.0$ & 411.2 & 86.0 \\
-17.81 & $l=0, \mathrm{~J}=2.0$ & 47.6 & 87.3 \\
\hline
\end{tabular}


Table 22. Thermal Characteristics $\left({ }^{141} \mathrm{Pr}\right)$

\begin{tabular}{|c|c|c|c|c|c|c|c|}
\hline Quantity & Unit & $\begin{array}{c}\text { BNL } \\
{[\mathrm{Mu81}]}\end{array}$ & $\begin{array}{c}\text { 98CRC } \\
\text { [Ho98] }\end{array}$ & $\begin{array}{c}\text { ENDF/ } \\
\text { B-VI }\end{array}$ & JEF-2.2 & $\begin{array}{c}\text { JENDL- } \\
3.2\end{array}$ & Present \\
\hline $\mathrm{R}^{\prime}$ & $\mathrm{fm}$ & $4.9 \pm 0.5$ & & 6.278 & 4.40 & 4.90 & 4.93 \\
$\mathrm{~B}$ & $\mathrm{fm}$ & $4.45 \pm 0.05$ & $4.58 \pm 0.05$ & & & & 4.58 \\
\hline$\sigma_{\gamma}^{0}$ & barn & $11.5 \pm 0.3$ & 11.5 & 11.5 & 11.5 & 11.5 & 11.5 \\
$\sigma_{\mathrm{s}}{ }^{0}$ & barn & $2.54 \pm 0.06$ & & 2.18 & 2.59 & 2.54 & 2.71 \\
\hline $\mathrm{gw}^{*}$ & & & & 1.002 & 0.9997 & 0.9990 & 0.9995 \\
\hline $\begin{array}{c}\mathrm{RI}- \\
\text { capt. }^{* *}\end{array}$ & barn & $17.4 \pm 2.0$ & $14 \pm 3$ & 19.0 & 17.9 & 18.4 & 17.6 \\
RI-total $^{* *}$ & barn & & & 303 & 234 & 239 & 237 \\
\hline
\end{tabular}

* Westcott factor for capture cross section.

** Integrated from $0.5 \mathrm{eV}$ to $100 \mathrm{keV}$ with $1 / \mathrm{E}$ spectrum.

Table 23. Average Resonance Parameters for Unresolved Resonance Region $\left({ }^{141} \mathrm{Pr}\right)$

\begin{tabular}{|c|c|c|c|c|c|c|c|}
\hline \multirow{2}{*}{ Quantity } & Unit & \multirow{2}{*}{$\begin{array}{c}\text { BNL } \\
\text { compilation }\end{array}$} & $\begin{array}{c}\text { ENDF/ } \\
\text { B-VI }\end{array}$ & JEF-2 & JENDL- & \multicolumn{2}{|c|}{ Present } \\
\cline { 6 - 8 } & & & & PT & Analysis & Adopted \\
\hline $\mathrm{R}^{\prime}$ & $\mathrm{fm}$ & $4.9 \pm 0.5$ & & 4.40 & 5.181 & & 4.93 \\
\hline$\left\langle\mathrm{D}_{0}\right\rangle$ & $\mathrm{eV}$ & $88 \pm 9$ & & 120.0 & 134.2 & $118.0 \pm 7.2$ & $118.0^{* *}$ \\
$\mathrm{~S}_{0}$ & $\times 10^{-4}$ & $1.5 \pm 0.2$ & & 1.00 & 1.50 & $1.77 \pm 0.30$ & 1.77 \\
$\left\langle\Gamma_{\gamma 0}\right\rangle$ & $\mathrm{meV}$ & & & 85 & 86 & & 86 \\
\hline$\left\langle\mathrm{D}_{1}\right\rangle$ & $\mathrm{eV}$ & & & & & $101.6 \pm 10$. & $59.0^{* *}$ \\
$\mathrm{~S}_{1}$ & $\times 10^{-4}$ & & & 62.2 & 67.1 & 9 & \\
$\left\langle\Gamma_{\gamma 1}\right\rangle$ & $\mathrm{meV}$ & & & 1.00 & 1.20 & $0.65 \pm 0.14$ & 0.75 \\
\hline$\left\langle\mathrm{D}_{2}\right\rangle$ & $\mathrm{eV}$ & & & 85 & 86 & & 86 \\
$\mathrm{~S}_{2}$ & $\times 10^{-4}$ & & & 44.1 & 44.7 & & $39.3 *$ \\
$\left\langle\Gamma_{\gamma 2}\right\rangle$ & $\mathrm{meV}$ & & & 3.574 & 1.50 & & 0.60 \\
\hline
\end{tabular}

* Average parameters at the low energy $(13.23 \mathrm{keV})$ of the unresolved region.

** Value at the neutron separation energy of ${ }^{142} \mathrm{Pr}$. 


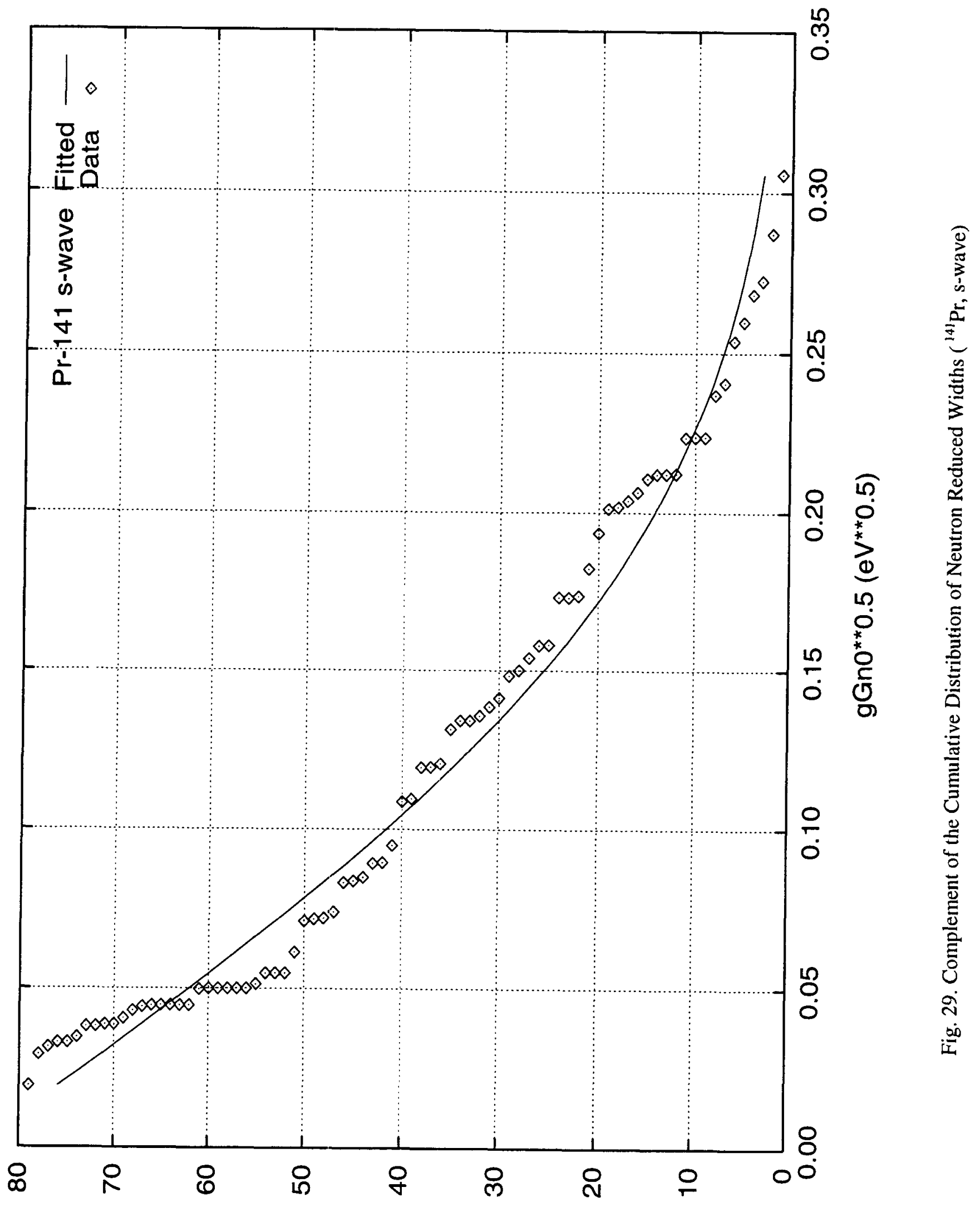

səoueuosay fo 'ON әм!̣e|nuno 


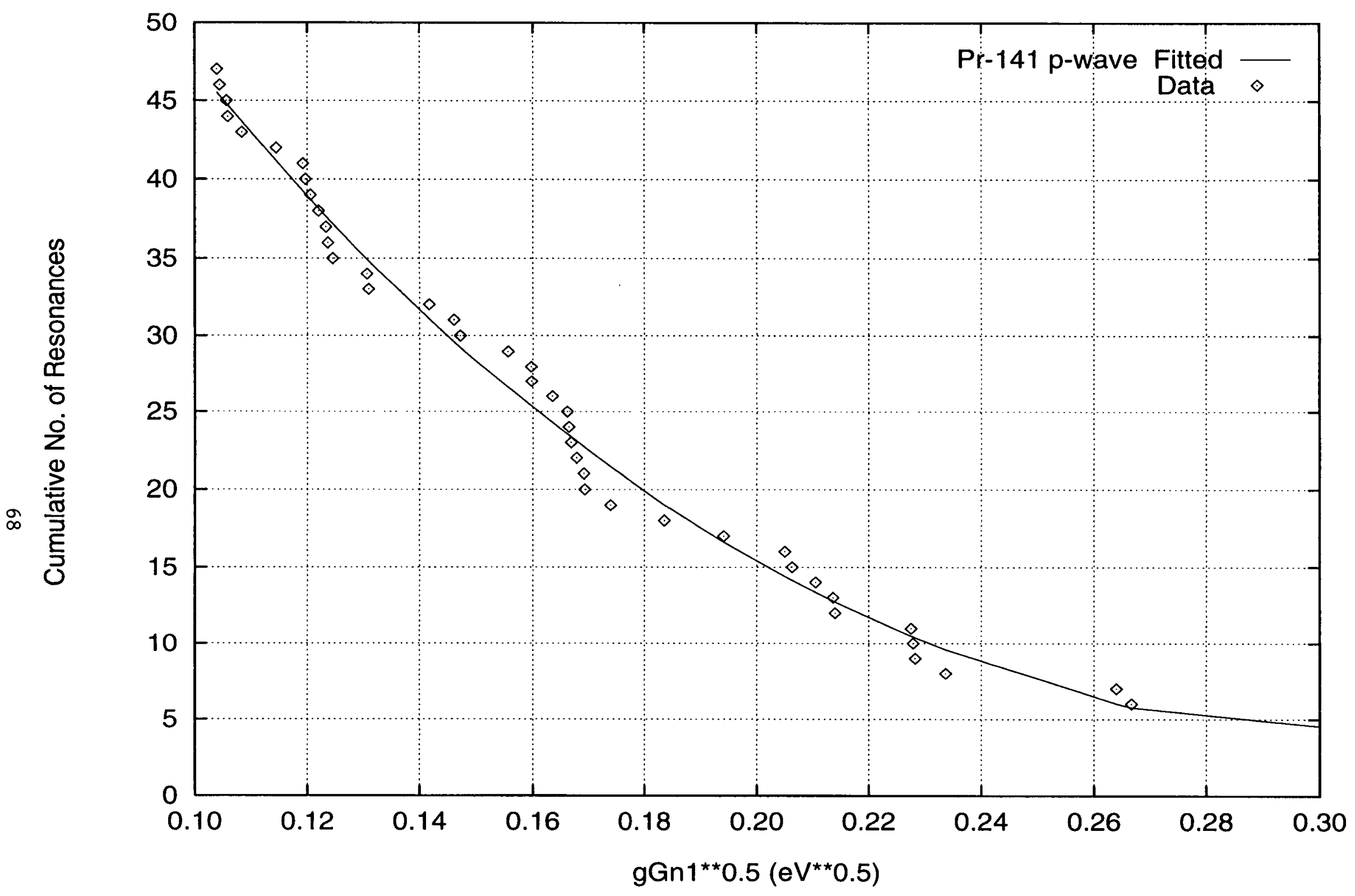

Fig. 30. Complement of the Cumulative Distribution of Neutron Reduced Widths ( ${ }^{141} \mathrm{Pr}, \mathrm{p}$-wave) 


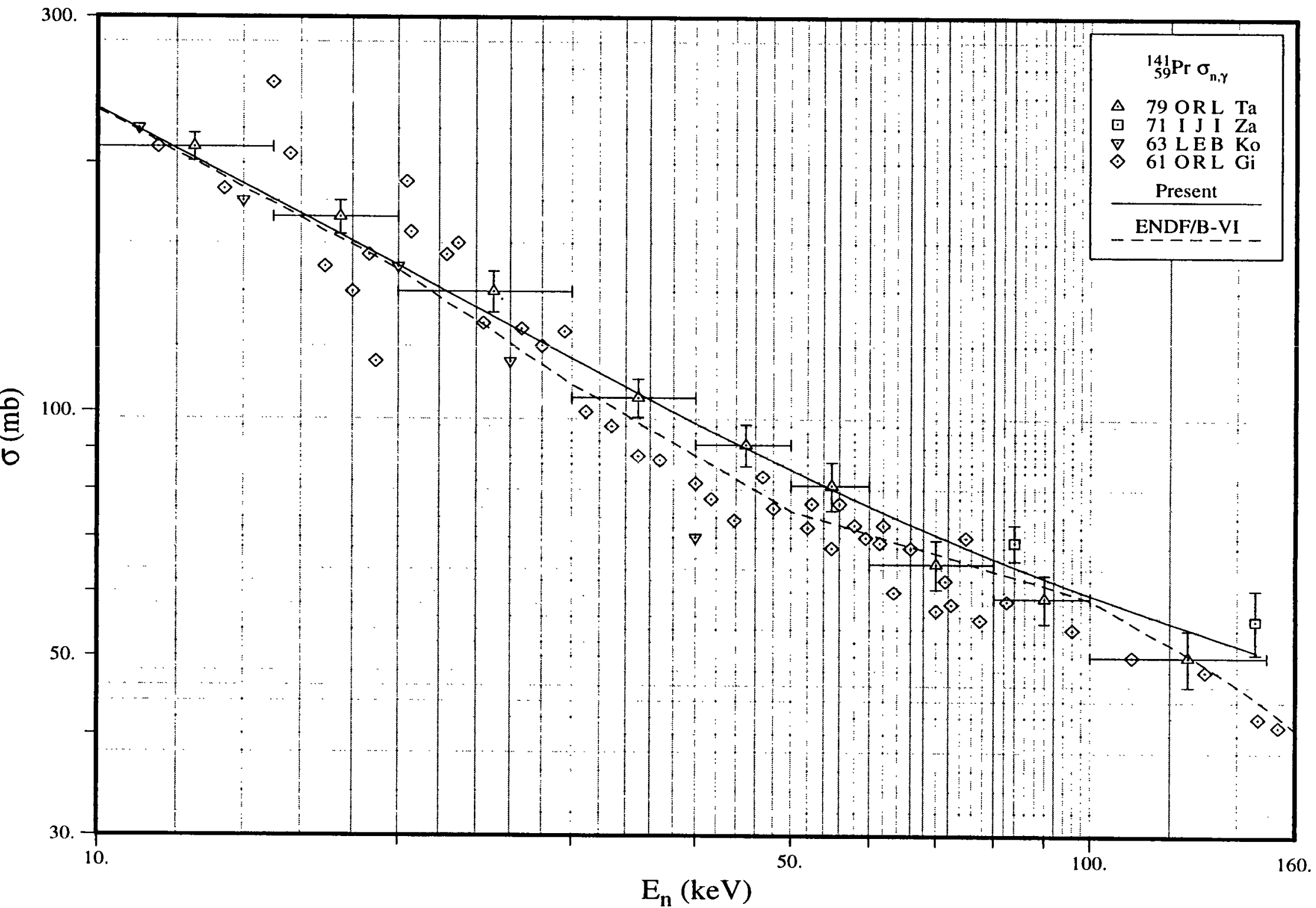

Fig. 31. Capture Cross Section in the Unresolved Resonance Region $\left({ }^{141} \mathrm{Pr}\right)$ 


\section{III.J. ${ }^{143} \mathrm{Nd}$}

1. Thermal Region

Since the contributions of positive energy resonances to the thermal capture cross section of ${ }^{143} \mathrm{Nd}$ is only 2.7 barns, a bound level is invoked to explain its thermal capture cross section, $325 \pm 10 \mathrm{~b}$ [Mu81]. The spin of the bound level is assumed as 3 on the basis of gamma-ray spectra measurements at thermal energy. The energy for a bound level is determined as $-6.5 \mathrm{eV}$ with a reduced neutron width of $104.7 \mathrm{meV}$ for an assumed radiative width of $72.3 \mathrm{meV}$. The Westcott factor for capture was calculated as 0.9966 . Table 24 summarizes thermal parameters and compares our results with other sources.

\section{Resolved Resonance Parameters}

The resonance parameters in the BNL compilation [Mu81] are mainly based on the data of Tellier at Saclay [Te71] and Musgrove et al. at ORNL [Mu77]. However, Allen et al. [A182a] reported that a correction factor is required for the ORNL data. A re-evaluation of the resonance parameters was carried out by reducing the reported capture areas by 0.9507 [Mu77] and recomputing $\Gamma_{\gamma}$ with the known $\mathrm{g} \Gamma_{\mathrm{n}}$ values. The spin assignments by Cauvin et al. [Ca71a] and Rohr et al. [Ro71] were adopted. Spins for other resonances were assigned randomly on the basis of the $(2 \mathrm{~J}+1)$ dependence of the level density.

On the basis of the Porter-Thomas distribution for all observed resonances (Fig. 32), an excess of weak resonances, interpreted as p-wave, was observed. The orbital angular momenta of resonances were determined by applying Bayesian analysis. Figures 33 and 34 display the Porter-Thomas distributions for $\mathrm{s}$ - and $\mathrm{p}$-wave resonances, respectively. The average resonance parameters obtained from this analysis are displayed in Table 25.

The upper limit of the resolved resonance region is set at $5503 \mathrm{eV}$.

\section{Unresolved Resonance Parameters}

Several capture measurements were carried out in the keV energy region. Musgrove et al. [Mu77] covered the energy region from $3 \mathrm{keV}$ to $100 \mathrm{keV}$. More recent measurement by Wisshak et al. [Wi98] covered the energy region from 3 to $225 \mathrm{keV}$. Other measurements, made by Yamamuro et al. at JAERI [Ya79] and Bokhovko et al. at Obninsk [Bo85], are significantly high at a few $\mathrm{keV}$ when compared with the data of Musgrove et al. [Mu77] and Wisshak et al. [Wi98].

As a starting point, the average resonance parameters obtained from the resolved resonance region were used. The level spacing was assumed to vary with energy and spin according to the Gilbert-Cameron's level density relation with associated parameters of [Mu98a] and a spin dispersion parameter of 3.5 [Mu98b]. In addition, p-wave strength function of 0.8 [Mu81] was assumed. P-wave average radiative width of $40 \mathrm{meV}$ and d-wave strength function of 1.5 were adopted from the systematics [Mu81]. These parameters resulted in a calculated capture cross section smaller than that of Wisshak et al. [Wi98] in the energy region up to 
several $\mathrm{keV}$. To achieve agreement with the data [Wi98], the s-wave average level spacing was adjusted to $36 \mathrm{eV}$ from a value of $38 \pm 2 \mathrm{eV}$. This adjusted value is within the uncertainty limits of that obtained from the Porter-Thomas distribution analysis. Figure 35 shows the resulting capture cross section, constructed from the average parameters that are listed in the last column of Table 25.

In addition to the $(n, \alpha)$ reaction, the neutron inelastic scattering to the first excited level is open at an energy of $747 \mathrm{keV}$. The upper energy limit of the unresolved resonance region was set at $225 \mathrm{keV}$.

Maxwellian average capture cross section for a $30 \mathrm{keV}$ temperature was computed as 239 $\mathrm{mb}$ for an upper energy limit of $1 \mathrm{MeV}$. From $225 \mathrm{keV}$ to $1 \mathrm{MeV}$, the point-wise capture cross section of the ENDF/B-VI evaluation was adopted. Our result is compared with a value of $244.6 \pm 3.1 \mathrm{mb}$ of Wisshak et al. [Wi98] and 242 $\pm 10 \mathrm{mb}$ reported in Beer's compilation [Be92].

Table 24. Thermal Parameters $\left({ }^{143} \mathrm{Nd}\right)$

\begin{tabular}{|c|c|c|c|c|c|c|c|}
\hline Quantity & Unit & $\begin{array}{c}\text { BNL } \\
\text { Compilation }\end{array}$ & $\begin{array}{c}\text { 98CRC } \\
\text { [Ho98] }\end{array}$ & $\begin{array}{c}\text { ENDF/B- } \\
\text { VI }\end{array}$ & JEF-2.2 & $\begin{array}{c}\text { JENDL- } \\
3.2\end{array}$ & Present \\
\hline$R^{\prime}$ & $\mathrm{fm}$ & & & 5.54 & 5.60 & 5.60 & 5.60 \\
$b^{\prime}$ & $\mathrm{fm}$ & & & 16.7 & & 17.3 & 16.7 \\
\hline$\sigma_{r}$ & barns & $325 \pm 10$ & $330 \pm 10$ & 325 & 323 & 330 & 325 \\
$\sigma_{s}$ & barns & $80 \pm 2$ & & 80.6 & 80.8 & 80.3 & 80.4 \\
\hline RI-capt. & barns & $128 \pm 30$ & $128 \pm 30$ & 130 & 130 & 130 & 130 \\
\hline
\end{tabular}


Table 25. Average Resonance Parameters in the Unresolved Resonance Region $\left({ }^{143} \mathrm{Nd}\right)$

\begin{tabular}{|c|c|c|c|c|c|c|c|}
\hline \multirow[b]{2}{*}{ Quantity } & \multirow[b]{2}{*}{ Unit } & \multirow[b]{2}{*}{$\begin{array}{c}\text { ENDF/B } \\
-\mathrm{VI}\end{array}$} & \multirow[b]{2}{*}{ JEF-2.2 } & \multirow[b]{2}{*}{$\begin{array}{c}\text { JENDL- } \\
3.2^{\mathrm{a}}\end{array}$} & \multirow[b]{2}{*}{$\begin{array}{c}\text { BNL } \\
\text { Compilation }\end{array}$} & \multicolumn{2}{|c|}{ Present } \\
\hline & & & & & & $\begin{array}{c}\text { PT } \\
\text { Analysis }\end{array}$ & Adopted \\
\hline$R^{\prime}$ (URR) & $\mathrm{fm}$ & 5.54 & 7.34 & 4.14 & & & 5.60 \\
\hline$<D_{0}>$ & $\mathrm{eV}$ & 32.5 & 36.0 & 36.4 & $45 \pm 4$ & $38.0 \pm 2.0$ & $36.0^{b}$ \\
\hline$S_{0}$ & $\times 10^{-4}$ & 3.5 & 3.4 & 2.62 & $3.2 \pm 0.3$ & $\begin{array}{c}3.62 \pm 0.5 \\
1\end{array}$ & 3.62 \\
\hline$<\Gamma_{y 0}>$ & $\mathrm{meV}$ & 80 & $76-68$ & 79.1 & $80 \pm 9$ & & 79.9 \\
\hline$\left.<D_{1}\right\rangle$ & $\overline{\mathrm{eV}}$ & 18.4 & 18.0 & 18.2 & & & $19.3^{b}$ \\
\hline$S_{1}$ & $\times 10^{-4}$ & 0.8 & 0.6 & 1.04 & $0.8 \pm 0.3$ & & 0.8 \\
\hline$\left\langle\Gamma_{r 1}\right\rangle$ & $\mathrm{meV}$ & 80 & $74 \sim 65$ & 79.1 & & & 40.0 \\
\hline$\left\langle D_{2}\right\rangle$ & $\mathrm{eV}$ & 12.3 & 12.0 & 12.1 & & & $14.5^{b}$ \\
\hline$S_{2}$ & $\times 10^{-4}$ & 1.0 & 3.0 & 1.78 & & & 1.50 \\
\hline$<\Gamma_{y 2}>$ & $\mathrm{meV}$ & 80 & $96 \sim 64$ & 79.1 & & & 79.9 \\
\hline
\end{tabular}

a: value at the low energy $(5 \mathrm{keV})$ of the unresolved energy region.

b: value at the neutron separation energy of ${ }^{144} \mathrm{Nd}$. 


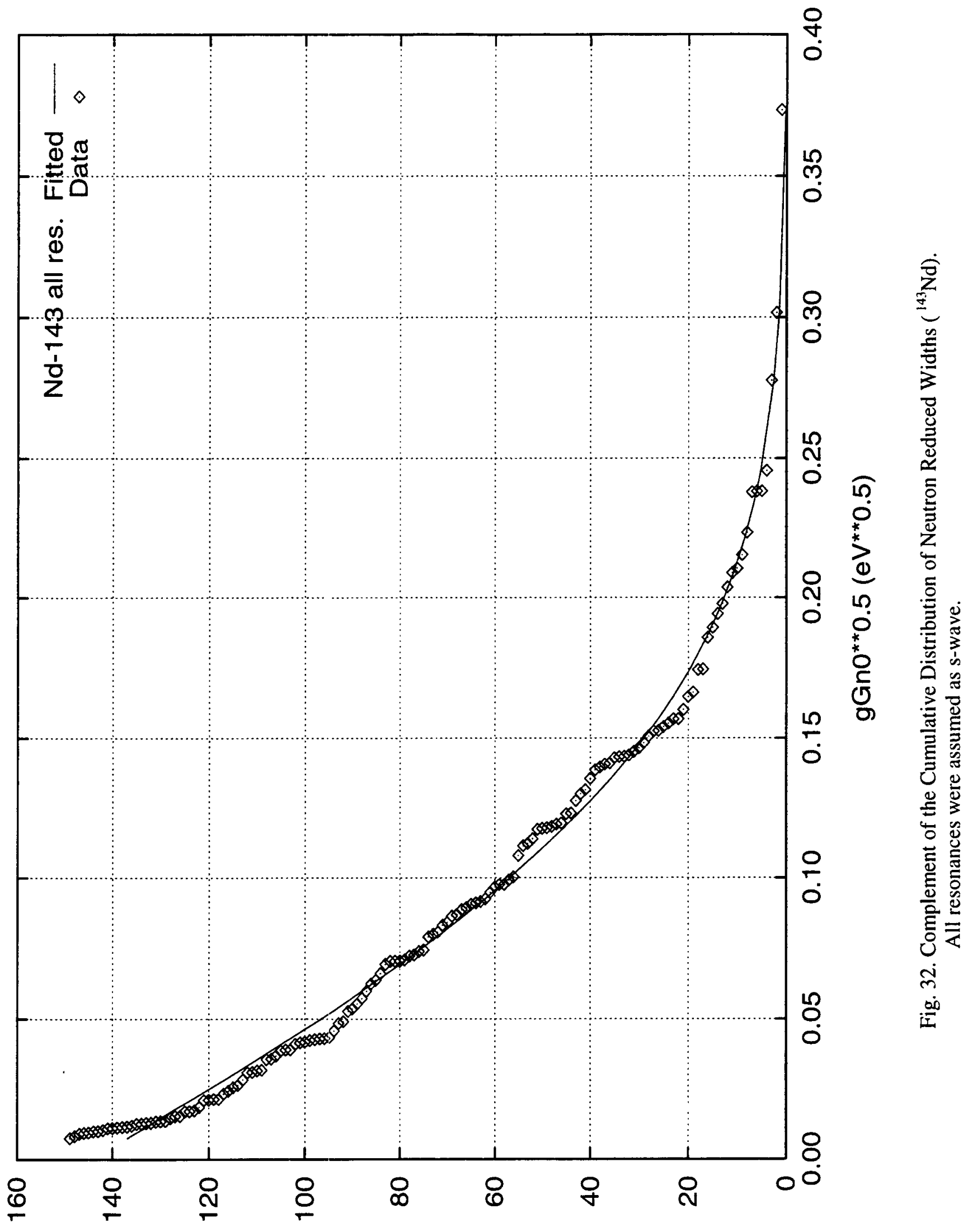

səoueuosey to "ON әм!̣e|numo 


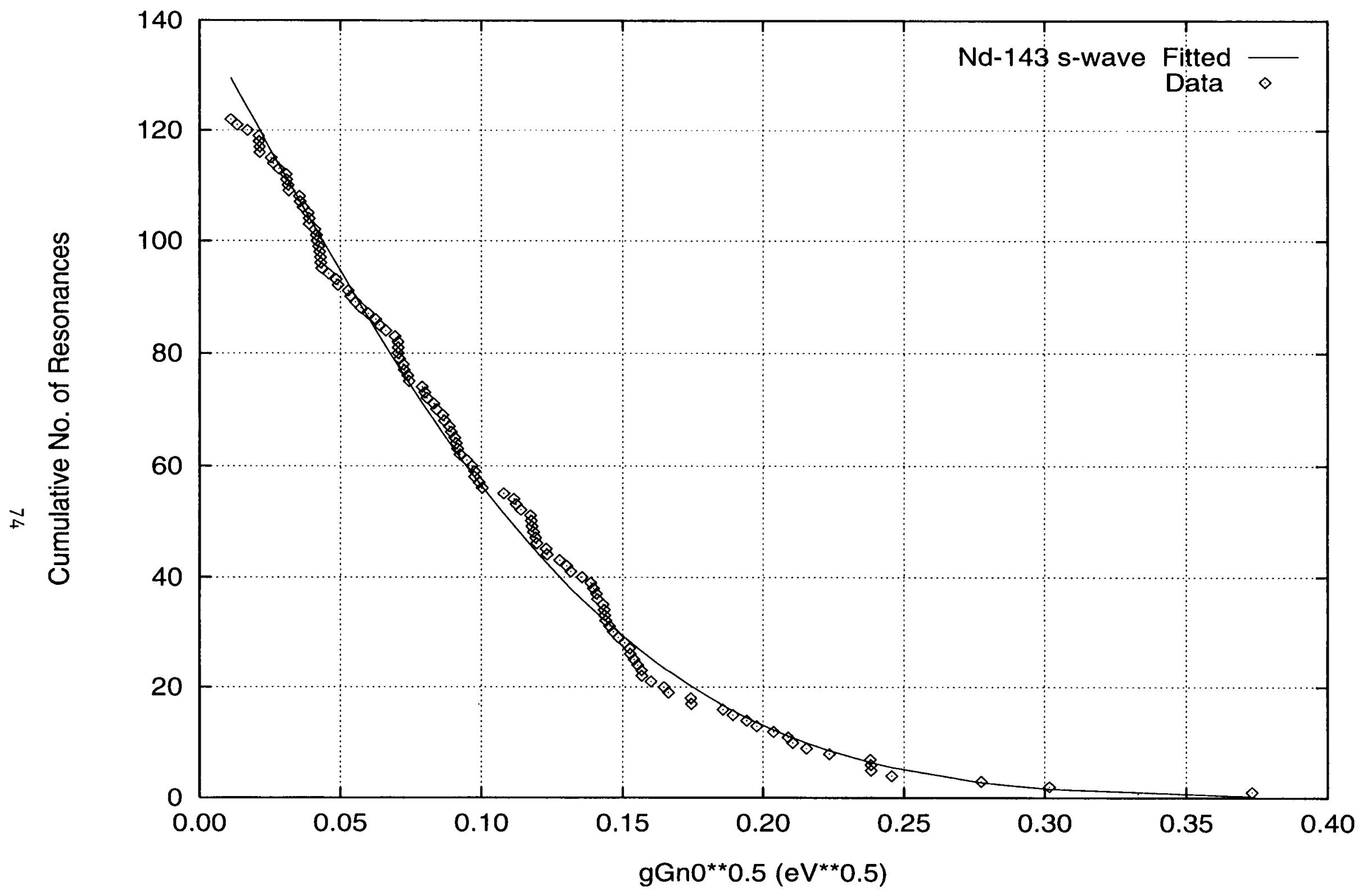

Fig. 33. Complement of the Cumulative Distribution of Neutron Reduced Widths ( ${ }^{143} \mathrm{Nd}, \mathrm{s}$-wave) 


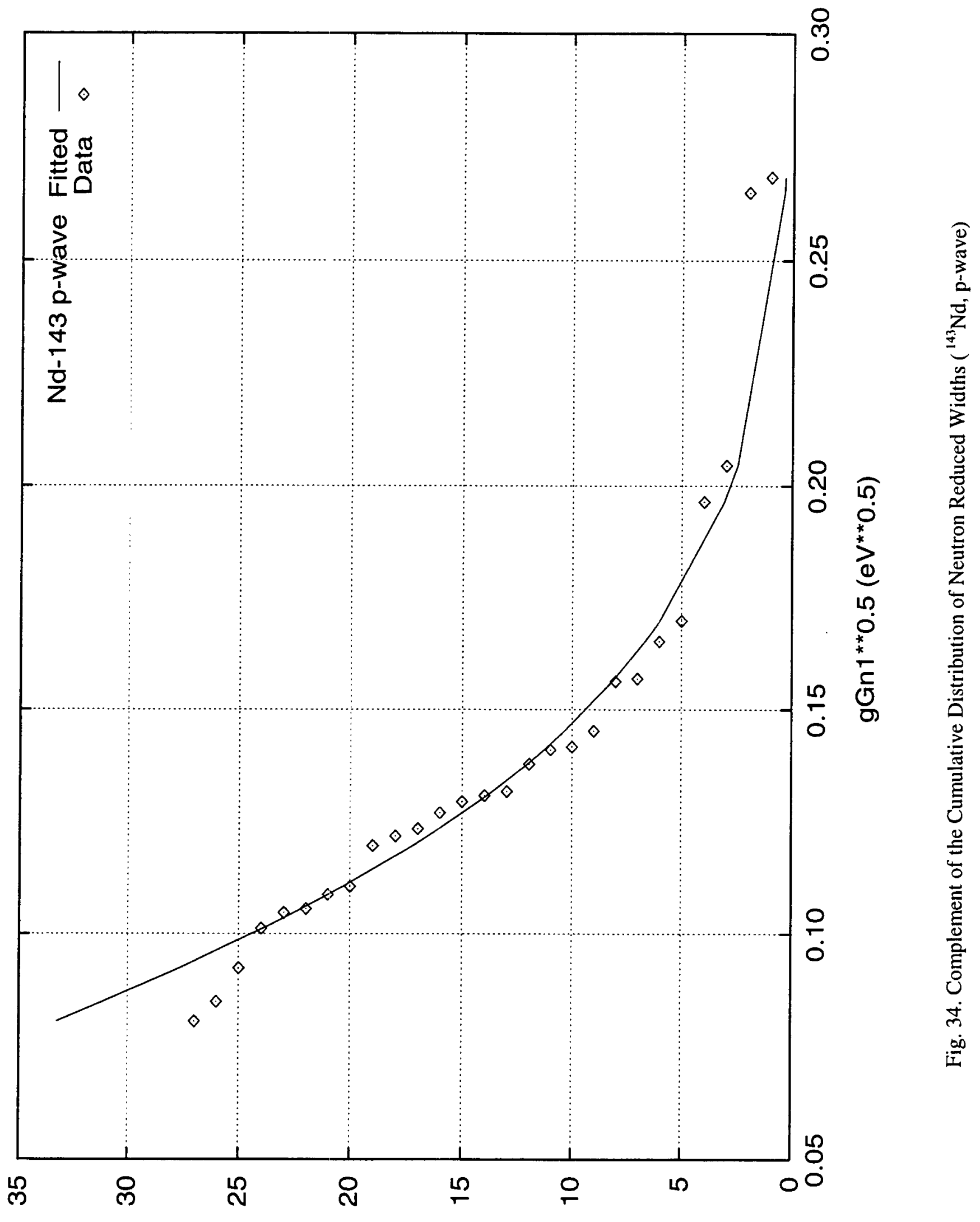

səoueuosey to 'ON əA!̣ejnuno 


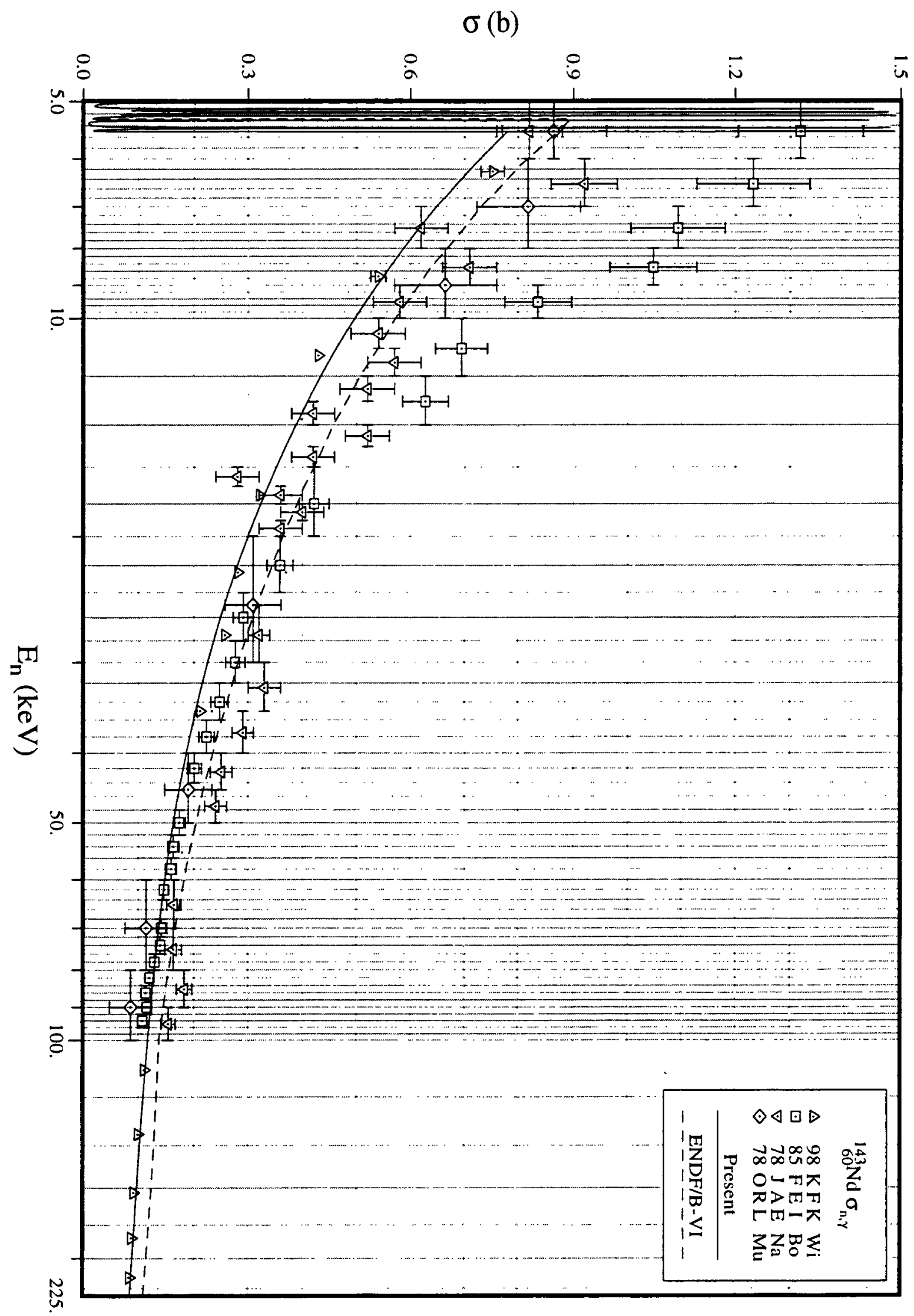




\section{III.K. ${ }^{145} \mathrm{Nd}$}

\section{Thermal Characteristics}

The thermal scattering and capture cross sections are evaluated by taking a weighted average of the results of Pattenden et al. [Pa58], Tattersall et al. [Ta60], Lucas et al. [Lu77], Vertebnyi et al. [Ve74], and Anufriev et al. [An79]. The present evaluated cross sections are included in Table 26 and are compared with other evaluations. A bound level is required to fit the capture and scattering cross sections in the thermal energy region. Its derived parameters are: $\mathrm{E}_{0}=-25.334 \mathrm{eV}, \mathrm{J}=3, \Gamma_{\mathrm{n}}=976.7 \mathrm{meV}$ for an assumed $\Gamma_{\gamma}=76.5 \mathrm{meV}$. The bound coherent scattering amplitude is computed as $8.73 \mathrm{fm}$ for an assumed potential scattering radius of 6.00 $\mathrm{fm}$, adopted from a deformed optical model calculation [Mu81]. The calculated Westcott factor for capture is 1.0000 .

\section{Resolved Resonance Parameters}

The evaluated resonance parameters [Mu81] are mainly based on the transmission data of Tellier at Saclay [Te71], the capture data of Rohr et al. at Geel [Ro71], and Musgrove et al. at ORNL [Mu77]. In evaluating the resonance parameters, the ORNL capture areas [Mu77] were decreased by a factor of 0.9507 as reported by Allen et al. [Al82]. A comparison of the scattering widths of the weak resonances of Rohr et al. [Ro71] with those of Tellier et al. [Te71], Karzhavina et al. [Ka69], and Anufriev et al. [An79] indicated that the capture areas of Rohr et $a l$. are low by about $20 \%$. This conclusion is also supported by the fact that the derived radiative widths of Rohr are low when compared with the results of Musgrove et al. [Mu77]. On this basis, the capture areas [Ro71] were increased by 20\%. The capture widths of the strong resonances were then recalculated with the aid of the known scattering widths [Te71]. For weak resonances, an average $\Gamma_{\gamma}=76.5 \mathrm{meV}$ was assumed. A weak resonance reported at $18.9 \mathrm{eV}$ [An79] is also included in the file.

Spin assignments, made by Cauvin et al. [Ca71a] and Stolovy et al. [St72], were considered. In addition, other spin assignments for strong resonances were made by us on the basis of the following criterion: resonances with a large capture area are assigned as $\mathrm{J}=4$ and those with a low value as $\mathrm{J}=3$. The scattering widths of weak resonances were calculated from the renormalized capture areas of Musgrove et al. [ $\mathrm{Mu} 77]$ for an assumed average radiative width of $76.5 \mathrm{meV}$ for the two possible spin values, 3 and 4. Subsequently, an average value was computed from the two results. The spins of the remaining resonances were assigned randomly on the basis of the $(2 \mathrm{~J}+1)$ law.

The analysis of reduced neutron widths for resonances below $4.0 \mathrm{keV}$ in terms of the Porter-Thomas distribution shows that few weak s-wave resonances are missed. A least squares fit, carried out for resonances having $\left(g \Gamma_{n}^{0}\right)^{1 / 2}>0.05 \mathrm{eV}$, is shown in Fig. 36. The average resonance parameters derived from this analysis are listed in Table 2. The Bayesian analysis shows that, with the exception of the $18.9 \mathrm{eV}$ resonance, all observed resonances are s-wave resonances. However, in the resonance parameter file, the weak resonance at $18.9 \mathrm{eV}$ was treated as an s-wave resonance. 


\section{Unresolved resonance parameters}

The low- and high-energy limits of the unresolved resonance region were set at $3979 \mathrm{eV}$ and $67.69 \mathrm{keV}$ respectively; the latter corresponds to the threshold energy for inelastic neutron scattering to the first excites state of ${ }^{145} \mathrm{Nd}$.

The capture data by Nakajima et al. at JAERI [Na78], Bokovko et al. at Obninsk [Bo85], and Wisshak et al. at Karlsruhe [Wi98] were considered in this evaluation. Wisshak et al.'s measurement covers the energy range from 3 to $225 \mathrm{keV}$.

The average s-wave resonance properties obtained from the resolved resonance analysis were used without adjustment. In addition, the $p$-wave average radiative width and the $d$-wave strength function were obtained from the systematics [Mu84]. The average parameters are summarized in Table 27. The energy-dependent level spacing of Gilbert-Cameron with the parameters of [Mu98a] and a spin dispersion parameter of 3.5 [Mu98b] was adopted. Our calculated capture cross section in the unresolved energy region is shown in Fig. 37 and is compared with the ENDF/B-VI evaluation and measured data. The present calculations strongly support Wisshak et al.'s measurement [Wi98]. Note that the JENDL-3.2 evaluation adopted Nakajima et al.'s measurement [Na78], which is high when compared with other measurements [Wi98, Bo85]. In addition, the average radiative widths for s-, p- and d-wave resonances in JENDL-3.2 are quite high when compared with our values (Table 27). The large s-wave radiative width of JENDEL-3.2 is not consistent with that obtained from the resolved energy region.

Maxwellian average capture cross section for a temperature of $30 \mathrm{keV}$ was computed as $423 \mathrm{mb}$. The calculation was carried out up to $1 \mathrm{MeV}$; the capture cross section was constructed with the present parameters up to $67.69 \mathrm{keV}$ and those of ENDF/B-VI from $67.69 \mathrm{keV}$ to $1 \mathrm{MeV}$. An excellent agreement was obtained with a value of $424.8 \pm 4.5 \mathrm{mb}$ [Wi98]; the reported value in the Beer's compilation is $485 \pm 100 \mathrm{mb}$ [Be92].

Table 26. Thermal Characteristics $\left({ }^{145} \mathrm{Nd}\right)$

\begin{tabular}{|c|c|c|c|c|c|c|c|}
\hline Quantity & Unit & $\begin{array}{c}\text { BNL } \\
\text { Compilation }\end{array}$ & $\begin{array}{c}\text { 98CRC } \\
\text { [Ho98] }\end{array}$ & ENDF/B-VI & JEF-2.2 & JENDL-3.2 & Present \\
\hline$R^{\prime}$ & $\mathrm{fm}$ & & & 6.8 & 6.0 & 6.0 & 6.0 \\
$b^{\prime}$ & $\mathrm{fm}$ & & & 9.9 & & & 8.73 \\
\hline$\sigma_{\gamma}$ & barns & $42 \pm 2$ & $47 \pm 6$ & 42.1 & 41.9 & 43.8 & 49.8 \\
$\sigma_{s}$ & barns & & & 17.4 & 18.3 & 20.3 & 18.7 \\
\hline RI-capt. & barns & $240 \pm 35$ & $260 \pm 40$ & 231 & 231 & 204 & 245 \\
\hline
\end{tabular}


Table 27. Average Resonance Parameters in the Unresolved Resonance Region $\left({ }^{145} \mathrm{Nd}\right)$

\begin{tabular}{|c|c|c|c|c|c|c|c|}
\hline \multirow{2}{*}{ Quantity } & Unit & ENDF/B & JEF-2.2 & JENDL- & BNL & \multicolumn{2}{|c|}{ Present } \\
\cline { 6 - 8 } & & & & BNompilation & P-T & Present \\
\hline$R^{\prime}(\mathrm{URR})$ & $\mathrm{fm}$ & 6.80 & 5.00 & 7.683 & & & 6.00 \\
\hline$\left\langle D_{0}\right\rangle$ & $\mathrm{eV}$ & 17.3 & 18.2 & 17.2 & $22 \pm 2$ & $18.0 \pm 0.8$ & $18.0^{\mathrm{b}}$ \\
$S_{0}$ & $\times 10^{-4}$ & 4.00 & 5.20 & 2.93 & $4.4 \pm 0.4$ & $4.75 \pm 0.52$ & 4.75 \\
$\left\langle\Gamma_{r 0}\right\rangle$ & $\mathrm{meV}$ & 75.0 & 89.1 & 97.5 & $75 \pm 9$ & & 76.5 \\
\hline$\left\langle D_{1}\right\rangle$ & $\mathrm{eV}$ & 8.66 & 9.40 & 8.61 & & & $9.64^{\mathrm{b}}$ \\
$S_{1}$ & $\times 10^{-4}$ & 0.80 & 0.98 & 0.69 & $0.8 \pm 0.4$ & - & 1.20 \\
$\left\langle\Gamma_{r 1}\right\rangle$ & $\mathrm{meV}$ & 75.0 & 88.1 & 97.5 & & & 40.0 \\
\hline$\left\langle D_{2}\right\rangle$ & $\mathrm{eV}$ & 5.78 & 6.60 & 5.74 & & & $7.25^{\mathrm{b}}$ \\
$S_{2}$ & $\times 10^{-4}$ & 1.00 & 3.90 & 3.51 & & & 0.80 \\
$\left\langle\Gamma_{r 2}\right\rangle$ & $\mathrm{meV}$ & 75.0 & 89.1 & 97.5 & & & 76.5 \\
\hline
\end{tabular}

a: value at energy $5.1 \mathrm{keV}$ of the unresolved energy region.

b: value at the neutron separation energy of ${ }^{146} \mathrm{Nd}$. 


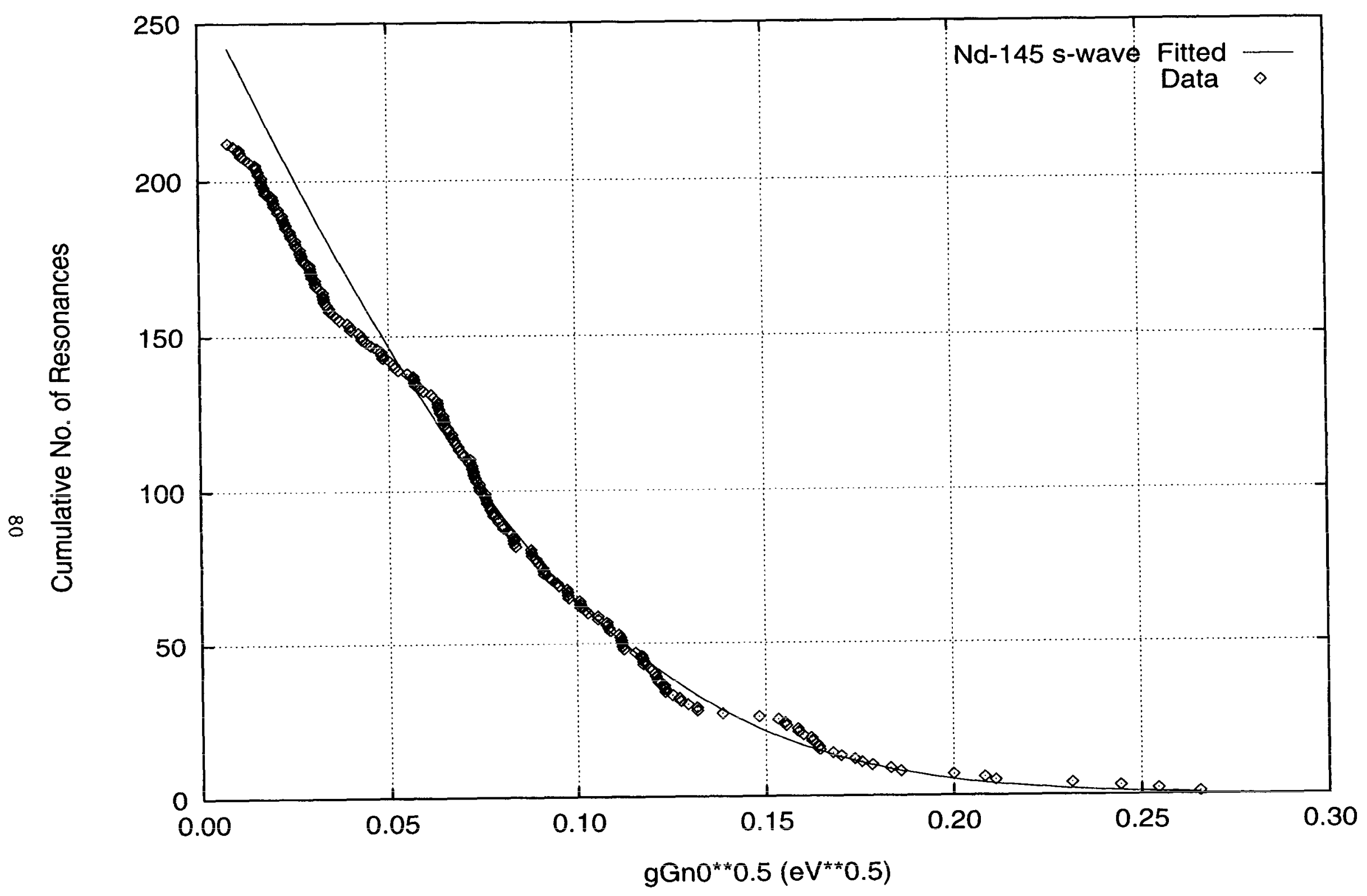

Fig. 36. Complement of the Cumulative Distribution of Neutron Reduced Widths ( ${ }^{145} \mathrm{Nd}$, s-wave) 


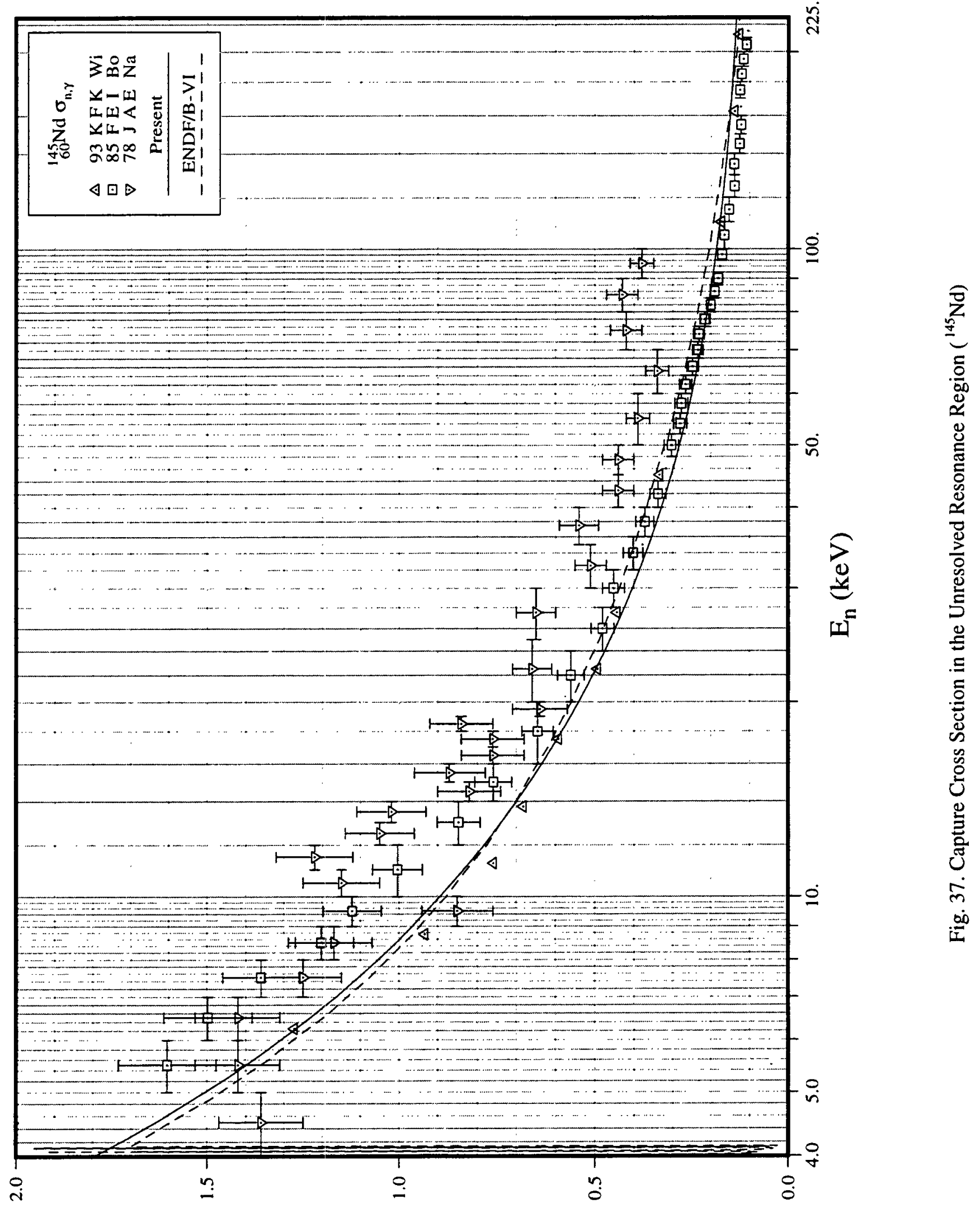

(q) $\mathrm{D}$ 


\section{III.L. ${ }^{147} \mathrm{Sm}$}

\section{Thermal Region}

Since the contribution of positive energy resonances to the thermal capture cross section is $21.4 \mathrm{~b}$, a bound level was invoked to reproduce a thermal capture cross section of $\sigma_{\gamma}{ }^{0}=50.0 \mathrm{~b}$. The latter value was obtained by re-normalizing the measurement of Fenner and Large [Fe67] $\left(\sigma_{\gamma}{ }^{0}=51 \pm 3 \mathrm{~b}, \mathrm{I}_{\gamma}=640 \pm 200 \mathrm{~b}\right)$ to a capture resonance integral of $\mathrm{I}_{\gamma}=700 \mathrm{~b}$. The bound coherent scattering length, $b_{\infty \circ h}=14 \pm 3 \mathrm{fm}$, recommended in the BNL compilation, was not considered in the determination of the bound level parameters for two reasons: a) a value of $14 \mathrm{fm}$ was previously deduced from the transmission measurement of Eiland et al. [Ei74] which suffered from a large uncertainty due to neutron absorption in the moisture and b) this large value of $b_{\text {coh }}$ resulted in unrealistic resonance parameters for the bound level. On the other hand, an effective scattering radius $R^{\prime}=8.3 \mathrm{fm}$, adopted from the BNL compilation [Mu84], resulted in the following parameters for the bound level: $E_{0}=-2.50 \mathrm{eV}, \mathrm{J}=4, \Gamma_{\mathrm{n}}=1.67 \mathrm{meV}, \Gamma_{\gamma}=73.8 \mathrm{meV}$. A comparison of the thermal cross sections and resonance integrals of the present evaluation with those of ENDF/B-VI, JEF-2.2, JENDL-3.2, and LIPAR-5 [Ab97] evaluations is presented in Table 28. With the exception of the capture cross section, the present quantities are in good agreement with those of JENDL-3.2 evaluation.

\section{Resolved Resonance Parameters}

The primary source of the resolved resonance parameters for this nuclide is the BNL compilation [Mu84]. In addition to this source, recent results of Georgiev et al. [Ge93] were incorporated into the present set of parameters. The present resolved energy region extends up to $1.998 \mathrm{keV}$. On the basis of Bayesian analysis, the observed 211 resonances were assigned as swave resonances. Resonance spins were assigned randomly to those resonances for which the spin is unknown. This random assignment assumed that the level density is proportional to $(2 \mathrm{~J}+1)$. The weighted averaging of 28 known radiative widths resulted in an average value of $73.8 \pm 1.5 \mathrm{meV}$, which was assumed for the remaining resonances with undetermined radiative widths. This average value is consistent with $69 \pm 2 \mathrm{meV}$ recommended in the BNL compilation [Mu84] and RIPL [Re98].

The distribution of reduced neutron widths was analyzed in terms of the Porter-Thomas distribution. Those resonances with reduced widths smaller than $1.0 \mathrm{meV}$ were excluded in the fitting procedure to obtain a best fit to the data. The fit, shown in Fig. 38, resulted in $\left\langle\mathrm{g} \Gamma_{\mathrm{n}}^{0}\right\rangle=2.94 \pm 0.29 \mathrm{meV}$ and $\left\langle\mathrm{D}_{0}\right\rangle=6.05 \pm 0.23 \mathrm{eV}$. These values yield an s-wave strength function, $S_{0}=4.86 \pm 0.50$. The present $\left\langle D_{0}\right\rangle$ is consistent with a value recommended in the BNL compilation [Mu84], $(5.7 \pm 0.5 \mathrm{eV})$, but is slightly higher than that recommended in the RIPL [Re98], $(5.1 \pm 0.5 \mathrm{eV})$. In addition, the present $S_{0}$ is consistent with a $4.8 \pm 0.5$ reported in the BNL compilation and RIPL. 


\section{Unresolved Resonance Parameters}

The present unresolved resonance region covers the energy range from $1.998 \mathrm{keV}$ to $122.05 \mathrm{keV}$. The latter energy corresponds to the threshold energy for inelastic neutron scattering to the first excited level of ${ }^{147} \mathrm{Sm}$ at $121.22 \mathrm{keV}$. For this nucleus, s-, $\mathrm{p}$ - and d-wave average resonance parameters were provided. The level spacing varies with energy according to Gilbert-Cameron level density formula with associated parameters adopted from Mughabghab and Dunford [Mu98a] (neutron separation energy $=8.14 \mathrm{MeV}$, pairing energy $=2.14 \mathrm{MeV}$, and level density parameter $\left.=17.5 \mathrm{MeV}^{-1}\right)$. The dependence of level spacing on $\exp \left\{-(\mathrm{J}+1 / 2)^{2} / 2 \sigma^{2}\right\}$, as well as $(2 \mathrm{~J}+1)$, was taken into account for this nucleus. With a value of $\sigma=3.4$ for the spin dispersion parameter [Mu98b], the level spacing for $\mathrm{p}$ - and d-wave resonances were calculated as $1 / 1.86$ and $1 / 2.47$ of the s-wave spacing, respectively. Note that these values deviate slightly from $1 / 2$ and $1 / 3$, obtained by assuming only $(2 \mathrm{~J}+1)$ dependence of the level spacing.

In the unresolved resonance region, the average cross sections measured by Wisshak et al. [Wi93], Macklin [Ma86], and Bokhovko et al. [Bo85] were taken into consideration. These measurements agree with each other, while the data sets of Kononov et al. [Ko77] and Mizumoto et al. [Mi81] deviate from the above measurements. As shown in Fig. 39, the present unresolved resonance parameters resulted in a good fit to the experimental data. The present parameters are listed in the last column of Table 29. The values of $\left\langle\Gamma_{\gamma}\right\rangle,\left\langle D_{0}\right\rangle$ and $S_{0}$ are those which resulted from the analysis in the resolved resonance region. The value of $S_{1}$ was adopted from a deformed optical model calculation [Mu84, Fig. 4], while $S_{2}$ was adjusted to 2.50 to reproduce the measured capture cross sections at the high energy limit. We note that $S_{1}$ is consistent with a value derived by Bokhovko et al. [Bo91] $\left(S_{1}=0.8 \pm 0.2\right)$, while $S_{2}$ deviates from those reported

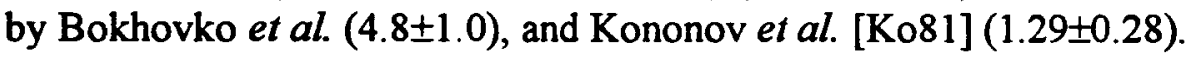

Maxwellian-averaged cross section for a temperature of $30 \mathrm{keV}$ was computed as 890 $\mathrm{mb}$. The present cross section is compared with $973.1 \pm 10 \mathrm{mb}$ of Wisshak et al. [Wi93] and $1005 \pm 100 \mathrm{mb}$ presented in Beer's compilation [Be92]. 
Table 28. Thermal Characteristics $\left({ }^{147} \mathrm{Sm}\right)$

\begin{tabular}{|c|c|c|c|c|c|c|c|c|}
\hline Quantity & Unit & $\begin{array}{c}\text { BNL } \\
\text { compilation }\end{array}$ & $\begin{array}{c}\text { 98CRC } \\
\text { [Ho98] }\end{array}$ & $\begin{array}{c}\text { ENDF/ } \\
\text { B-VI }\end{array}$ & $\begin{array}{c}\text { JEF- } \\
2.2\end{array}$ & $\begin{array}{c}\text { JENDL- } \\
3.2\end{array}$ & $\begin{array}{c}\text { LIPAR-5 } \\
\text { [Ab97] }\end{array}$ & Present \\
\hline $\mathrm{R}^{\prime}$ & $\mathrm{fm}$ & $8.3 \pm 0.2$ & & 8.30 & 8.30 & 8.30 & & 8.30 \\
\hline$\sigma_{\gamma}{ }^{0}$ & barn & $57 \pm 3$ & $56 \pm 4$ & 57.5 & 57.2 & 58.0 & 56.7 & 50.0 \\
$\sigma_{\mathrm{s}}{ }^{0}$ & barn & & & 39.1 & 39.9 & 1.06 & 3.8 & 1.06 \\
\hline $\mathrm{gw}^{*}$ & & & & 0.9990 & 1.001 & 0.9946 & 0.9942 & 0.9965 \\
\hline RI-capt. & barn & & $710 \pm 50$ & 789 & 793 & 779 & 721 & 777 \\
RI-total & barn & & & 1660 & 1639 & 1524 & & 1505 \\
\hline
\end{tabular}

*Westcott factor for capture cross section.

Table 29. Average Resonance Parameters for the Unresolved Resonance Region $\left({ }^{147} \mathrm{Sm}\right)$

\begin{tabular}{|c|c|c|c|c|c|c|c|}
\hline \multirow[b]{2}{*}{ Quantity } & \multirow[b]{2}{*}{ Unit } & \multirow{2}{*}{$\begin{array}{c}\text { BNL } \\
\text { compilation }\end{array}$} & \multirow{2}{*}{$\begin{array}{l}\text { ENDF/ } \\
\text { B-VI }\end{array}$} & \multirow[b]{2}{*}{ JEF-2.2 } & \multirow{2}{*}{$\begin{array}{c}\text { JENDL- } \\
3.2\end{array}$} & \multicolumn{2}{|c|}{ Present } \\
\hline & & & & & & $\begin{array}{c}\text { PT } \\
\text { Analysis }\end{array}$ & Adopted \\
\hline $\mathbf{R}^{\prime}$ & $\mathrm{fm}$ & $8.3 \pm 0.2$ & 8.30 & 6.37 & 6.64 & & 8.30 \\
\hline$\left\langle\overline{D_{0}}\right\rangle$ & $\overline{\mathrm{eV}}$ & $5.7 \pm 0.5$ & 5.70 & 6.32 & 5.70 & $6.05 \pm 0.23$ & $6.05^{*}$ \\
\hline $\mathrm{S}_{0}$ & $\times 10^{-4}$ & $4.8 \pm 0.5$ & 4.80 & 4.26 & 4.80 & $4.86 \pm 0.50$ & 4.86 \\
\hline$\left\langle\Gamma_{\gamma 0}\right\rangle$ & $\mathrm{meV}$ & $69 \pm 2$ & 69.0 & 87.6 & 69.0 & & 73.8 \\
\hline$\left\langle\overline{\left.D_{1}\right\rangle}\right.$ & $\mathrm{eV}$ & & 2.85 & 3.25 & 2.85 & & $3.25^{*}$ \\
\hline $\mathrm{S}_{1}$ & $\times 10^{-4}$ & & 0.65 & 1.75 & 1.00 & & 0.80 \\
\hline$\left\langle\Gamma_{\gamma_{1}}\right\rangle$ & $\mathrm{meV}$ & & 69.0 & 87.6 & 69.0 & & 40.0 \\
\hline$\left\langle\mathrm{D}_{2}\right\rangle$ & $\overline{\mathrm{eV}}$ & & & 2.28 & 1.90 & & $2.45^{*}$ \\
\hline $\mathrm{S}_{2}$ & $\times 10^{-4}$ & & & 6.50 & 4.70 & & 2.50 \\
\hline$\left\langle\Gamma_{\gamma_{2}}\right\rangle$ & $\mathrm{meV}$ & & & 87.6 & 69.0 & & 73.8 \\
\hline
\end{tabular}

${ }^{*}$ Value at the neutron separation energy of ${ }^{148} \mathrm{Sm}$. 


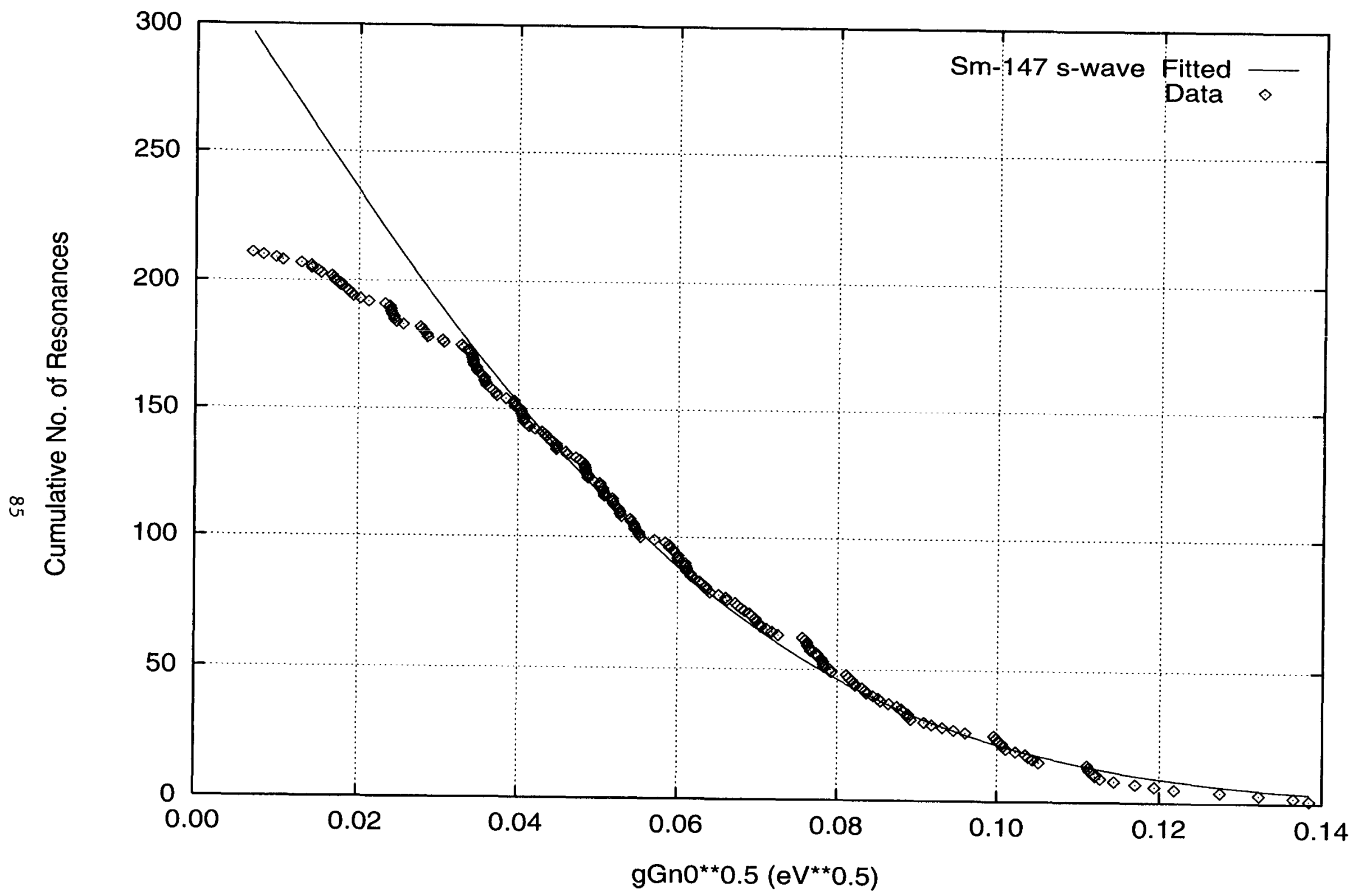

Fig. 38. Complement of the Cumulative Distribution of Neutron Reduced Widths ( ${ }^{147} \mathrm{Sm}, \mathrm{s}$-wave) 


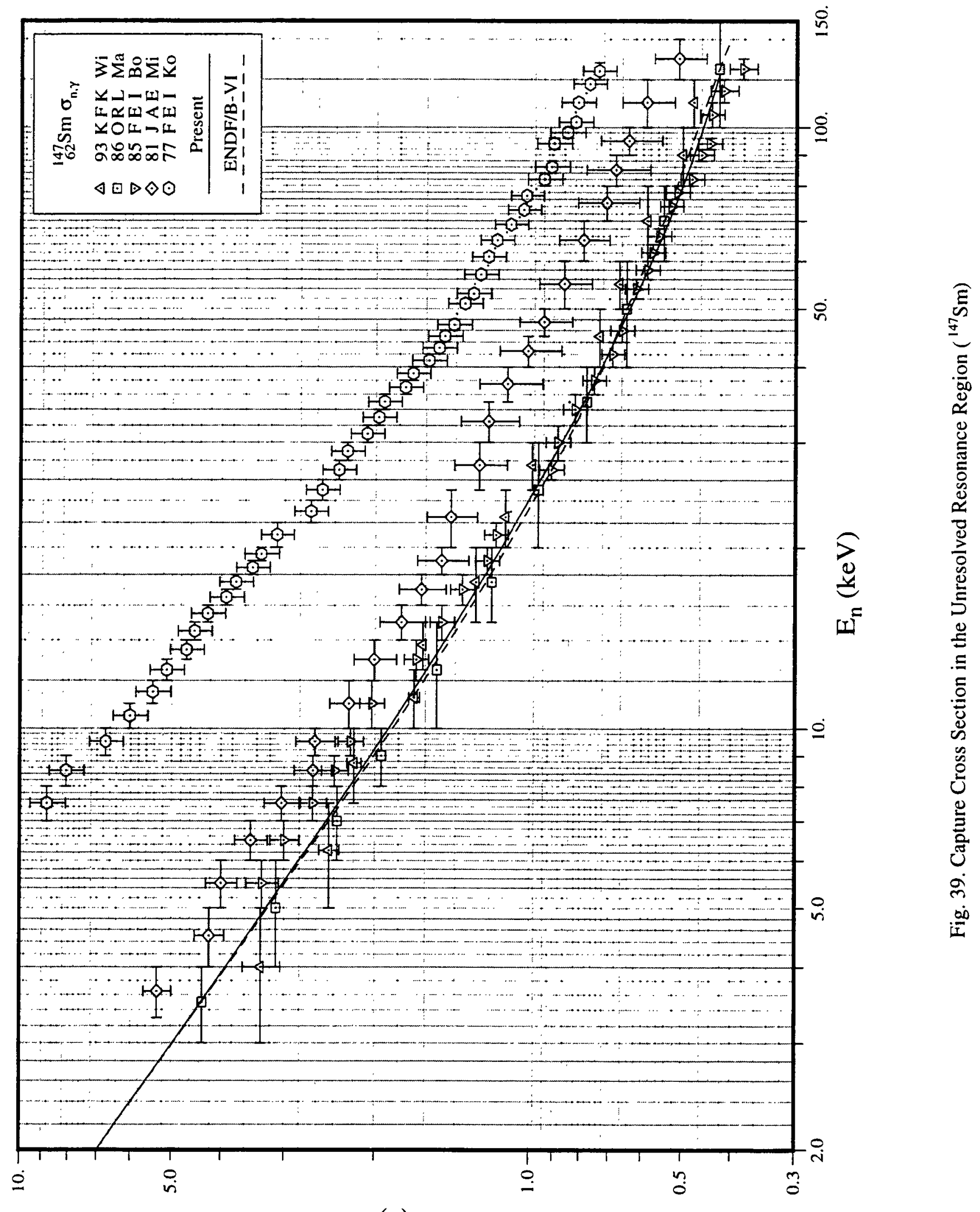

(q) 0 


\section{III.M. ${ }^{149} \mathrm{Sm}$}

\section{Thermal Cross Sections}

The thermal capture cross section is adopted from the Mughabghab's BNL compilation [Mu84]. The spin of the bound level is assigned as 3 according to the $(n, \alpha)$ measurements of Dakowski [Da67]. The energy and reduced neutron width of the bound level were determined as $1.5 \mathrm{eV}$ and $7.243 \mathrm{meV}$, respectively. Since the first resonance at $0.0923 \mathrm{eV}$ is close to the thermal energy, the coherent scattering length $(b)$ varies strongly with energy. Word and Werner [Wo82] measured the energy dependence of the bound coherent scattering length in the energy range from 0.02945 to $0.09274 \mathrm{eV}$. Subsequently, Lynn and Seeger [Ly90] carried out R-matrix analysis in the low energy region by considering only the resonance at $0.09274 \mathrm{eV}$. The influence of other positive energy resonances was taken into account by energy dependent background terms. The R-matrix calculation [Ly90], represented by a dashed line in Fig. 40, is compared with measurement [Wo82]. By applying multi-level Breit-Wigner formalism [Mu81, Eq.3], we have also carried out computations of the energy-dependence of the $\operatorname{Re}\left(b^{\prime}\right)$ for two cases. In one case, represented by a solid line, the effect of the bound level, as well as all positive energy resonances, was considered. In the second, denoted by a dotted line, the effect of the bound level was excluded. As shown in Fig. 40, our results support the important contribution of the bound level.

Our results, listed in Table 30, are in very good agreement with the reference values, reported in the BNL conpilation [Mu84], but are discrepant with those of the ENDF/B-VI evaluation. The present Westcott factor for capture is calculated as 1.7171.

\section{Resolved Resonance Parameters}

Recent scattering, capture, and gamma-ray multiplicity measurements by Georgiev [Ge92] determined radiative widths and spins of resonances in the energy region $15.85-272.8$ $\mathrm{eV}$. These resonance parameters, along with the scattering widths of the BNL compilation of [Mu84], were incorporated into a new evaluation of the resolved resonance parameters.

The weighted-average capture width of 61 resonances is determined as $56.7 \pm 8.9 \mathrm{meV}$. This result seems quite low, because of the dominant weights of the first two resonances at $0.0973 \mathrm{eV}$ and $0.872 \mathrm{eV}$. However, we opted to adopt an un-weighted average of $78.5 \mathrm{meV}$.

All the observed resonances were assigned as s-wave according to Bayesian analysis. The Porter-Thomas distribution shows that many weak s-wave resonances are missed. A leastsquares fit for values of $\left(g \Gamma_{n}^{0}\right)^{1 / 2}>0.024$, represented by a solid line, is shown in Fig. 41. The average resonance parameters, determined from this analysis, are displayed in Table $\mathbf{3 1}$ and are compared with other evaluations. The derived s-wave parameters are to be compared also with those reported in RIPL [Re98]: $\left\langle\mathrm{D}_{0}\right\rangle=2.10 \pm 0.30 \mathrm{eV}, \mathrm{S}_{0}=6.30 \pm 1.20$, and $\left\langle\Gamma_{\gamma}\right\rangle=62 \pm 2 \mathrm{meV}$.

The upper energy limit of the resolved resonance region was set to $520 \mathrm{eV}$. 


\section{Unresolved resonance parameters}

The average s-wave resonance parameters, obtained from the resolved resonance region, were applied without adjustments in the unresolved energy region. The $p$ - and $d$-wave strength functions, as well as the p-wave average radiative width, were adopted from the systematics [Mu84]. The average parameters, implemented in the unresolved energy region, are summarized in Table 31 and are compared with other evaluations. The level spacing was assumed to vary with energy according to Gilbert-Cameron level density relation [Mu98a].

Neutron inelastic scattering to the first excited level of ${ }^{149} \mathrm{Sm}$ is open at an energy of $22.507 \mathrm{keV}$. The upper energy limit of the unresolved resonance region was set to $100 \mathrm{keV}$. Competition widths in the energy region from $22.5 \mathrm{keV}$ to $100 \mathrm{keV}$ were not provided. The energy of the second excited level is $277.075 \mathrm{keV}$. The capture cross section, constructed from the present parameters, is compared in Fig. 42 with measurements of Wisshak et al. [Wi93], Macklin [Ma86], Kononov et al. [Ko77] and Hockenberry et al.

Maxwellian average capture cross section for a temperature of $30 \mathrm{keV}$, computed as 1866 $\mathrm{mb}$, is to be compared with $1819.9 \pm 17.2 \mathrm{mb}$ [Wi93] and $1409 \pm 95 \mathrm{mb}$ [Be92]. The calculation was extended up to $1 \mathrm{MeV}$. The capture cross section was constructed with the present parameters up to an energy of $100 \mathrm{keV}$; from $100 \mathrm{keV}$ to $1 \mathrm{MeV}$, it was borrowed from the ENDF/B-VI evaluation.

Table 30. Thermal Characteristics $\left({ }^{149} \mathrm{Sm}\right)$

\begin{tabular}{|c|c|c|c|c|c|c|c|}
\hline Quantity & Unit & $\begin{array}{c}\text { BNL } \\
\text { Compilation }\end{array}$ & $\begin{array}{c}\text { 98CRC } \\
\text { [Ho98] }\end{array}$ & $\begin{array}{c}\text { ENDF/ } \\
\text { B-VI }\end{array}$ & JEF-2.2 & $\begin{array}{c}\text { JENDL- } \\
3.2\end{array}$ & Present \\
\hline$R^{\prime}$ & $\mathrm{fm}$ & $8.3 \pm 0.2$ & & 5.093 & 8.30 & 7.52 & 8.30 \\
$\operatorname{Re}\left(b^{\prime}\right)$ & $\mathrm{fm}$ & -24 & $-19.08^{*}$ & -24.2 & & -24.1 & -19.08 \\
\hline$\sigma_{r}$ & barns & $40140 \pm 600$ & $40100 \pm 600$ & 39730 & 40480 & 40150 & 40530 \\
$\sigma_{s}$ & barns & 197 & & 139 & 176 & 173 & 194 \\
\hline RI-capt. & barns & 3390 & $3310 \pm 500$ & 3258 & 3484 & 3490 & 3482 \\
\hline
\end{tabular}

* Value of Lynn [Ly90]. 
Table 31. Average Resonance Parameters in the Unresolved Resonance Region $\left({ }^{149} \mathrm{Sm}\right)$

\begin{tabular}{|c|c|c|c|c|c|c|c|}
\hline \multirow[b]{2}{*}{ Quantity } & \multirow[b]{2}{*}{ Unit } & \multirow{2}{*}{$\begin{array}{c}\text { ENDF/B- } \\
\text { VI }\end{array}$} & \multirow{2}{*}{ JEF-2.2 } & \multirow{2}{*}{$\begin{array}{c}\text { JENDL- } \\
3.2^{2}\end{array}$} & \multirow{2}{*}{$\begin{array}{c}\text { BNL } \\
\text { Compilation }\end{array}$} & \multicolumn{2}{|c|}{ Present } \\
\hline & & & & & & $\begin{array}{c}\mathrm{PT} \\
\text { Analysis }\end{array}$ & Adopted \\
\hline$R^{\prime}$ (URR) & $\mathrm{fm}$ & 5.093 & 5.09 & 7.90 & $8.3 \pm 0.2$ & & 8.30 \\
\hline$<D_{0}>$ & $\mathrm{eV}$ & 3.42 & 1.97 & 1.54 & $2.2 \pm 0.2$ & $2.45 \pm 0.11$ & $2.45^{b}$ \\
\hline$S_{0}$ & $\times 10^{-4}$ & 3.20 & 4.13 & 4.60 & $4.6 \pm 0.6$ & $4.53 \pm 0.54$ & 4.53 \\
\hline$\left\langle\Gamma_{y 0}\right\rangle$ & $\mathrm{meV}$ & 62.0 & 75.1 & 62.0 & $62 \pm 2$ & & 78.5 \\
\hline$<D_{1}>$ & $\mathrm{eV}$ & 1.71 & 1.01 & $0.77^{+}$ & & & $1.31^{\mathrm{b}}$ \\
\hline$S_{1}$ & $\times 10^{-4}$ & 0.50 & 2.32 & 0.30 & $0.3 \pm 0.1$ & & 1.00 \\
\hline$\left.<\Gamma_{\gamma 1}\right\rangle$ & $\mathrm{meV}$ & 62.0 & 75.1 & 62.0 & & & 40.0 \\
\hline$<D_{2}>$ & $\mathrm{eV}$ & & 0.707 & $0.514^{+}$ & & & $0.987^{b}$ \\
\hline$S_{2}$ & $\times 10^{-4}$ & & 6.50 & 4.90 & & & 4.00 \\
\hline$\left\langle\mathrm{r}_{\gamma_{2}}\right\rangle$ & $\mathrm{meV}$ & & 75.1 & 62.0 & & & 78.5 \\
\hline
\end{tabular}

a: At the low energy of the unresolved resonance region, $0.52 \mathrm{keV}$.

b: At the neutron separation energy of ${ }^{150} \mathrm{Sm}$. 


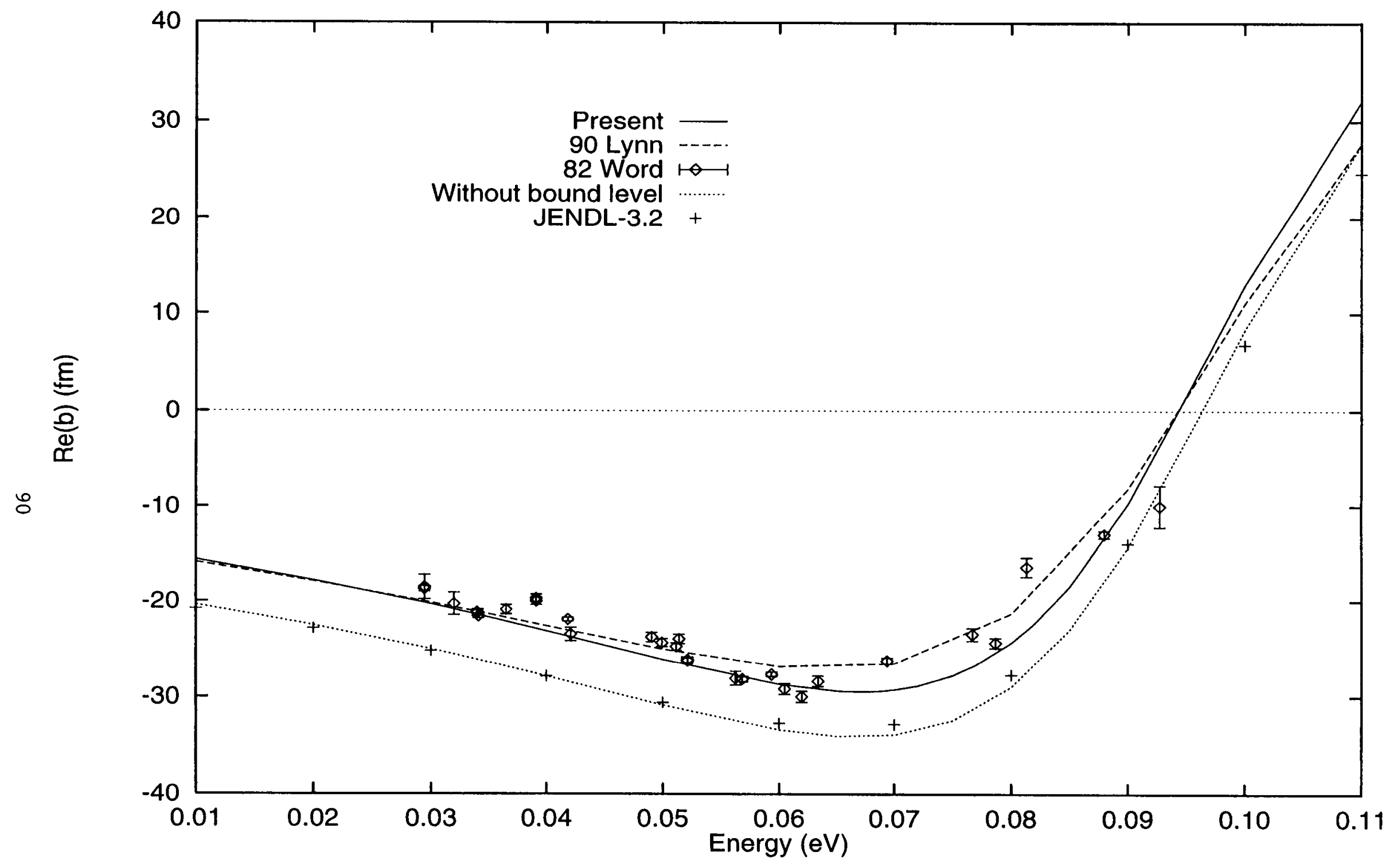

Fig. 40. Real Part of the Bound Coherent Scattering Length of ${ }^{149} \mathrm{Sm}$ 


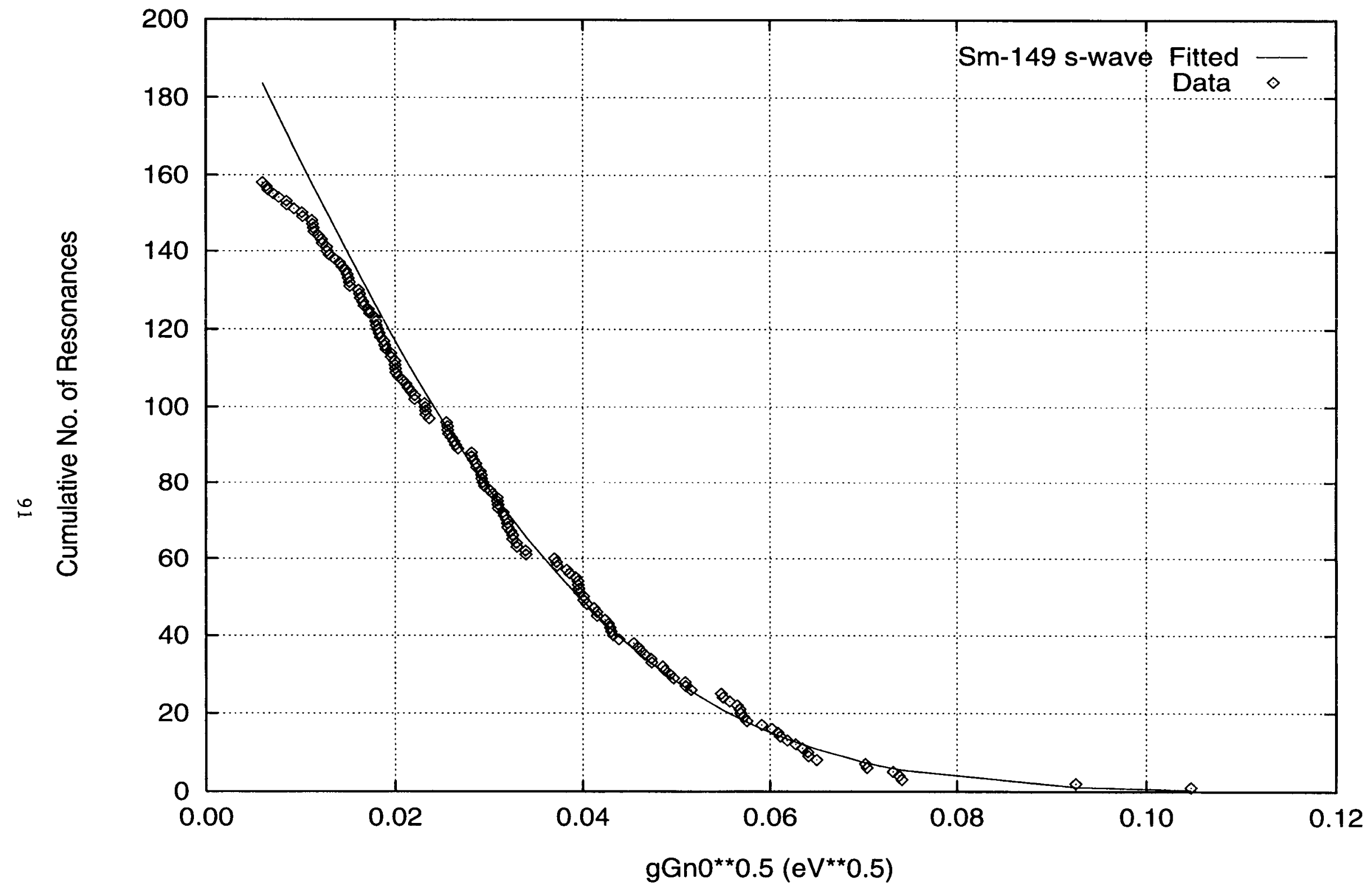

Fig. 41. Complement of the Cumulative Distribution of Neutron Reduced Widths ( ${ }^{149} \mathrm{Sm}$, s-wave) 


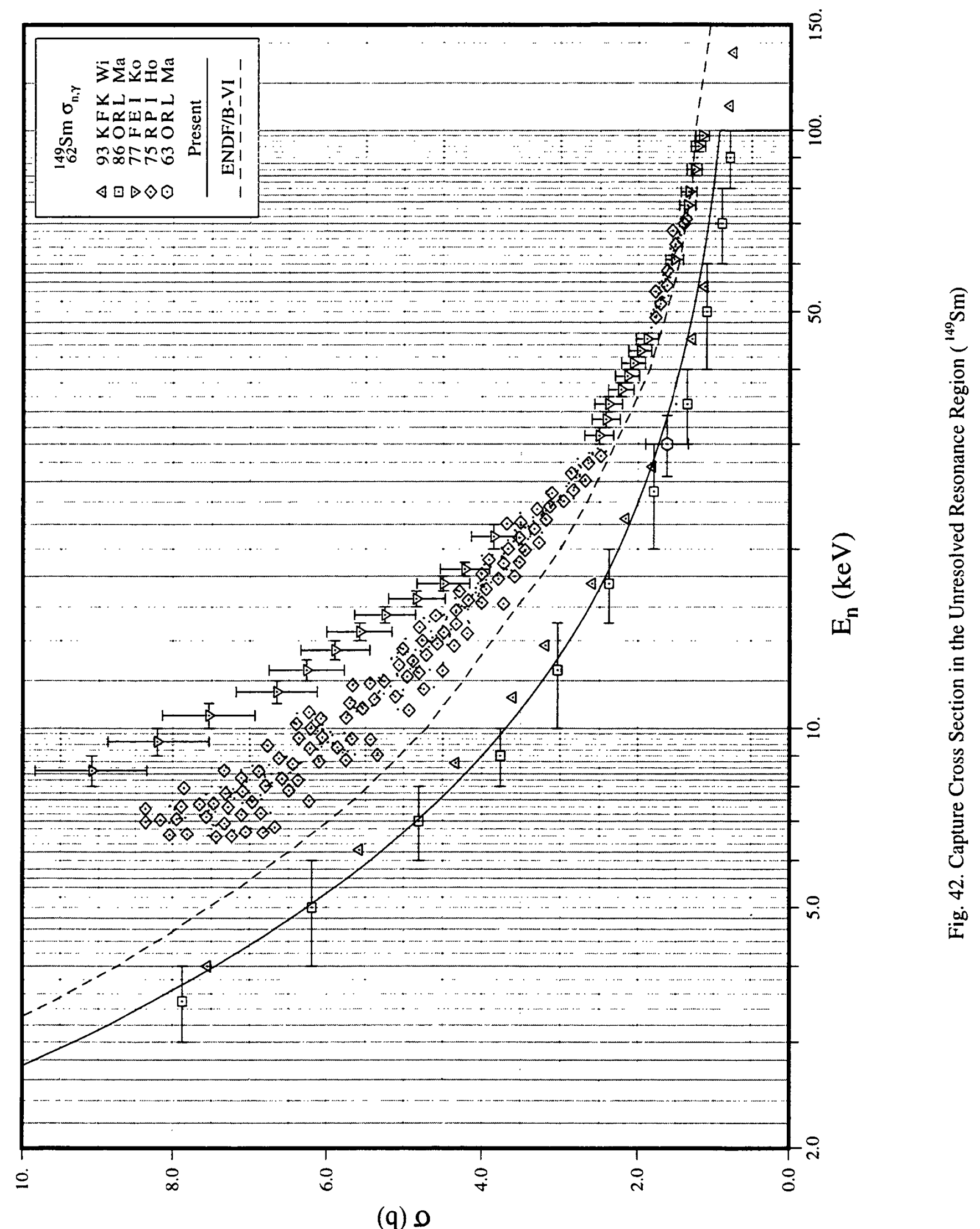


III.N. ${ }^{150} \mathrm{Sm}$

1. Thermal Region

To reproduce a thermal capture cross section of $100 \mathrm{~b}$, as well as a thermal elastic scattering cross section of $27.6 \mathrm{~b}$, one bound level was invoked. The reference thermal capture cross section was obtained by weighting Maxwellian-averaged values $97 \pm 10 \mathrm{~b}$ of Halperin et al. [Ha62] and $101 \pm 5 \mathrm{~b}$ of Aitken and Cornish [Ai61]. The latter value is adjusted from the original value of $102 \pm 5 \mathrm{~b}$ on the basis of a revised value for the resonance integral. The reference scattering cross section was obtained by subtracting the capture cross section from the total cross section, 127.5 b [Ei74], in which a paramagnetic scattering cross section of $7.5 \mathrm{~b}$ [Ei74] was excluded. The bound resonance parameters are; $\mathrm{E}_{0}=-11.72 \mathrm{eV}, \Gamma_{\mathrm{n}}=176.6 \mathrm{meV}, \Gamma_{\gamma}=60.2$ $\mathrm{meV}$. In these calculations, an effective scattering radius ( $\left.\mathrm{R}^{\prime}\right)$ of $8.0 \mathrm{fm}$ was adopted from a deformed optical model calculation [Mu81, Fig. 2]. The contribution of positive energy resonances to the thermal capture cross section was calculated as $69.5 \mathrm{~b}$. The bound coherent scattering length was calculated as $14.8 \mathrm{fm}$ ( $4.7 \mathrm{fm}$ without a bound level), which is consistent with a value of $14 \pm 3 \mathrm{fm}$ in the BNL compilation [Mu84].

A comparison of the various thermal cross sections and resonance integrals of the present evaluation and those of ENDF/B-VI, JEF-2 and JENDL-3 is presented in Table 32. While there is general agreement between the various evaluations of the thermal capture cross sections and capture resonance integrals, our evaluated scattering cross section deviates from those in the other cross section libraries.

\section{Resolved Resonance Parameters}

Because measurements were not carried out since the date of the BNL compilation, the resolved resonance parameters of the present evaluation were based on [Mu84].

On the basis of Bayesian analysis, the reported 22 resonances [Mu84] were assigned as swave. An average radiative width of $60 \pm 5 \mathrm{meV}$, based on [Mu84], was assumed for all resonances except for the first two positive-energy resonances for which radiative widths were determined experimentally [Mu84]. It is noted that this average value is much smaller than $87 \pm 16 \mathrm{meV}$ reported in RIPL [Re98].

The distributions of reduced neutron widths were analyzed in terms of the Porter-Thomas distribution. Resonances with reduced widths smaller than $3 \mathrm{meV}$ were excluded in the fitting procedure to obtain a minimum $\chi^{2}$ value. Figure 43 shows the fit to the data, which resulted in $\left\langle g \Gamma_{\mathrm{n}}{ }^{9}\right\rangle=20.1 \pm 6.6 \mathrm{meV}$ and $\left\langle\mathrm{D}_{0}\right\rangle=46.5 \pm 8.6 \mathrm{eV}$. These values yield s-wave strength function of $\mathrm{S}_{0}=4.31 \pm 1.42$. The present $\left\langle\mathrm{D}_{0}\right\rangle$ is consistent with values recommended in the BNL compilation [Mu84] (55 $\pm 9 \mathrm{eV})$ and RIPL [Re98] $(46 \pm 8 \mathrm{eV})$ within the associated uncertainties.

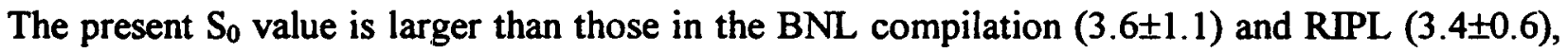
but consistent with them within the uncertainties. Because of the limited number of resonances, the uncertainties of the derived average quantities are rather large. 


\section{Unresolved Resonance Parameters}

The present unresolved resonance region covers the energy range from $1.563 \mathrm{keV}$ to $336.1 \mathrm{keV}$. The latter energy corresponds to neutron inelastic scattering to the first excited level of ${ }^{150} \mathrm{Sm}$ at $333.86 \mathrm{keV}$. For this nuclide, s-, $\mathrm{p}$ - and d-wave average resonance parameters were provided. The level spacing varies with energy according to Gilbert-Cameron level density formula in which the associated constants were adopted from Mughabghab and Dunford [Mu98a, Mu98b] (neutron separation energy $=5.596 \mathrm{MeV}$, pairing energy $=1.22 \mathrm{MeV}$, and level density parameter $\left.=21.76 \mathrm{MeV}^{-1}\right)$. The dependence of level spacing on $\exp \left\{-(\mathrm{J}+1 / 2)^{2} / 2 \sigma^{2}\right\}$, as well as $(2 \mathrm{~J}+1)$, was taken into account for this nucleus. With a value of $\sigma=3.7$ for the spin dispersion parameter [Mu98b], the level spacings for $p$ - and d-wave resonances are calculated as $1 / 2.78$ and $1 / 4.04$ of the s-wave spacing, respectively. These values deviate from $1 / 3$ and $1 / 5$, obtained by assuming only a $(2 \mathrm{~J}+1)$ dependence of the level spacing.

In the unresolved resonance region, cross sections measurements carried out by Wisshak et al. [Wi93], Winters et al. [Wi86], and Macklin et al. [Ma63] were taken into consideration. The data of Kononov et al. [Ko78] deviate significantly from the others. As shown in Fig. 44, our computed capture cross section is in very good agreement with the experimental data [Wi93, Wi78]. The present average resonance parameters are listed in the last column of Table 33. Values of $\left\langle D_{0}\right\rangle$ and $S_{0}$ are those which were derived from the analysis of the resolved region. The present value of $S_{1}$ was adopted from a deformed optical model calculation [Mu84, Fig. 4], while that of $S_{2}$ was adjusted to reproduce the capture cross section measured by Wisshak et al. [Wi93] above $50 \mathrm{keV}$. An average radiative width of $60 \mathrm{meV}$ was assumed for s- and d-wave resonances. A p-wave radiative width of $40 \mathrm{meV}$ was determined in the present analysis to reproduce the capture cross section in the low energy region. This value is consistent with the systematics of $\mathrm{p}$-wave radiative widths around mass number of 150 [Mu84, Fig. 7].

Table 32. Thermal Characteristics $\left({ }^{150} \mathrm{Sm}\right)$

\begin{tabular}{|c|c|c|c|c|c|c|c|}
\hline Quantity & Unit & $\begin{array}{c}\text { BNL } \\
\text { compilation }\end{array}$ & $\begin{array}{l}\text { 98CRC } \\
\text { [Ho98] }\end{array}$ & $\begin{array}{c}\text { ENDF/ } \\
\text { B-VI }\end{array}$ & JEF-2.2 & $\begin{array}{l}\text { JENDL- } \\
3.2\end{array}$ & Present \\
\hline $\mathbf{R}^{\prime}$ & $\mathrm{fm}$ & & & 6.41 & 6.40 & 8.08 & 8.00 \\
\hline $\begin{array}{l}\sigma_{\gamma}^{0} \\
\sigma_{s}{ }^{0}\end{array}$ & $\begin{array}{l}\text { barn } \\
\text { barn }\end{array}$ & $104 \pm 4$ & $102 \pm 5$ & $\begin{array}{c}104 \\
19.0\end{array}$ & $\begin{array}{c}103 \\
21.2\end{array}$ & $\begin{array}{c}109 \\
8.34\end{array}$ & $\begin{array}{l}100 \\
27.7\end{array}$ \\
\hline $\mathrm{gw}_{\mathrm{w}}^{*}$ & & & & 1.000 & 0.9979 & 0.9938 & 0.9985 \\
\hline $\begin{array}{c}\text { RI- } \\
\text { capt. }^{* *} \\
\text { RI-total }^{* *}\end{array}$ & $\begin{array}{l}\text { barn } \\
\text { barn }\end{array}$ & $358 \pm 50$ & $290 \pm 30$ & $\begin{array}{l}338 \\
830\end{array}$ & $\begin{array}{l}339 \\
840\end{array}$ & $\begin{array}{l}325 \\
799\end{array}$ & $\begin{array}{l}334 \\
890\end{array}$ \\
\hline
\end{tabular}

* Westcott factor for capture cross section.

** Integrated from $0.5 \mathrm{eV}$ to $100 \mathrm{keV}$ with $1 / \mathrm{E}$ spectrum. 
Table 33. Average Resonance Parameters for the Unresolved Resonance Region $\left({ }^{150} \mathrm{Sm}\right)$

\begin{tabular}{|c|c|c|c|c|c|c|c|}
\hline \multirow[b]{2}{*}{ Quantity } & \multirow[b]{2}{*}{ Unit } & \multirow{2}{*}{$\begin{array}{c}\text { BNL } \\
\text { compilation }\end{array}$} & \multirow{2}{*}{$\begin{array}{l}\text { ENDF/ } \\
\text { B-VI }\end{array}$} & \multirow[b]{2}{*}{ JEF-2.2 } & \multirow[b]{2}{*}{$\begin{array}{l}\text { JENDL- } \\
3.2^{*}\end{array}$} & \multicolumn{2}{|c|}{ Present } \\
\hline & & & & & & $\begin{array}{c}\text { PT } \\
\text { Analysis }\end{array}$ & Adopted \\
\hline $\mathbf{R}^{\prime}$ & $\mathrm{fm}$ & & 6.41 & & 5.92 & & 8.00 \\
\hline$\left\langle\mathrm{D}_{0}\right\rangle$ & $\overline{\mathrm{eV}}$ & $55 \pm 9$ & 48.0 & & 59.8 & $46.5 \pm 8.6$ & $46.5^{* *}$ \\
\hline $\mathrm{S}_{0}$ & $\times 10^{-4}$ & $3.6 \pm 1.1$ & 3.60 & & 3.60 & $4.31 \pm 1.42$ & 4.31 \\
\hline$\left\langle\Gamma_{\gamma 0}\right\rangle$ & $\mathrm{meV}$ & $60 \pm 5$ & 60.0 & & 60.0 & & 60.0 \\
\hline$\left\langle\mathrm{D}_{1}\right\rangle$ & $\mathrm{eV}$ & & 15.0 & & 20.0 & & $16.7^{* *}$ \\
\hline$S_{1}$ & $\times 10^{-4}$ & $0.08 \pm 0.02$ & 0.60 & & 1.40 & & 1.00 \\
\hline$\left\langle\Gamma_{\gamma_{1}}\right\rangle$ & $\mathrm{meV}$ & & 60.0 & & 60.0 & & 40.0 \\
\hline$\left\langle\mathrm{D}_{2}\right\rangle$ & $\overline{\mathrm{eV}}$ & & 6.00 & & 12.0 & & $11.5^{* *}$ \\
\hline$S_{2}$ & $\times 10^{-4}$ & & 2.90 & & 2.30 & & 3.50 \\
\hline$\left\langle\Gamma_{\gamma_{2}}\right\rangle$ & $\mathrm{meV}$ & & 60.0 & & 60.0 & & 60.0 \\
\hline
\end{tabular}

* Average parameters at the low energy bound of the unresolved resonance region $(1.54 \mathrm{keV})$. ** At the neutron separation energy of ${ }^{151} \mathrm{Sm}$.

Maxwellian average capture cross section for a temperature of $30 \mathrm{keV}$, computed as 409 $\mathrm{mb}$, is to be compared with $421.9 \pm 3.8 \mathrm{mb}$ [Wi93] and 434 $\pm 26 \mathrm{mb}$ [Be92]. 


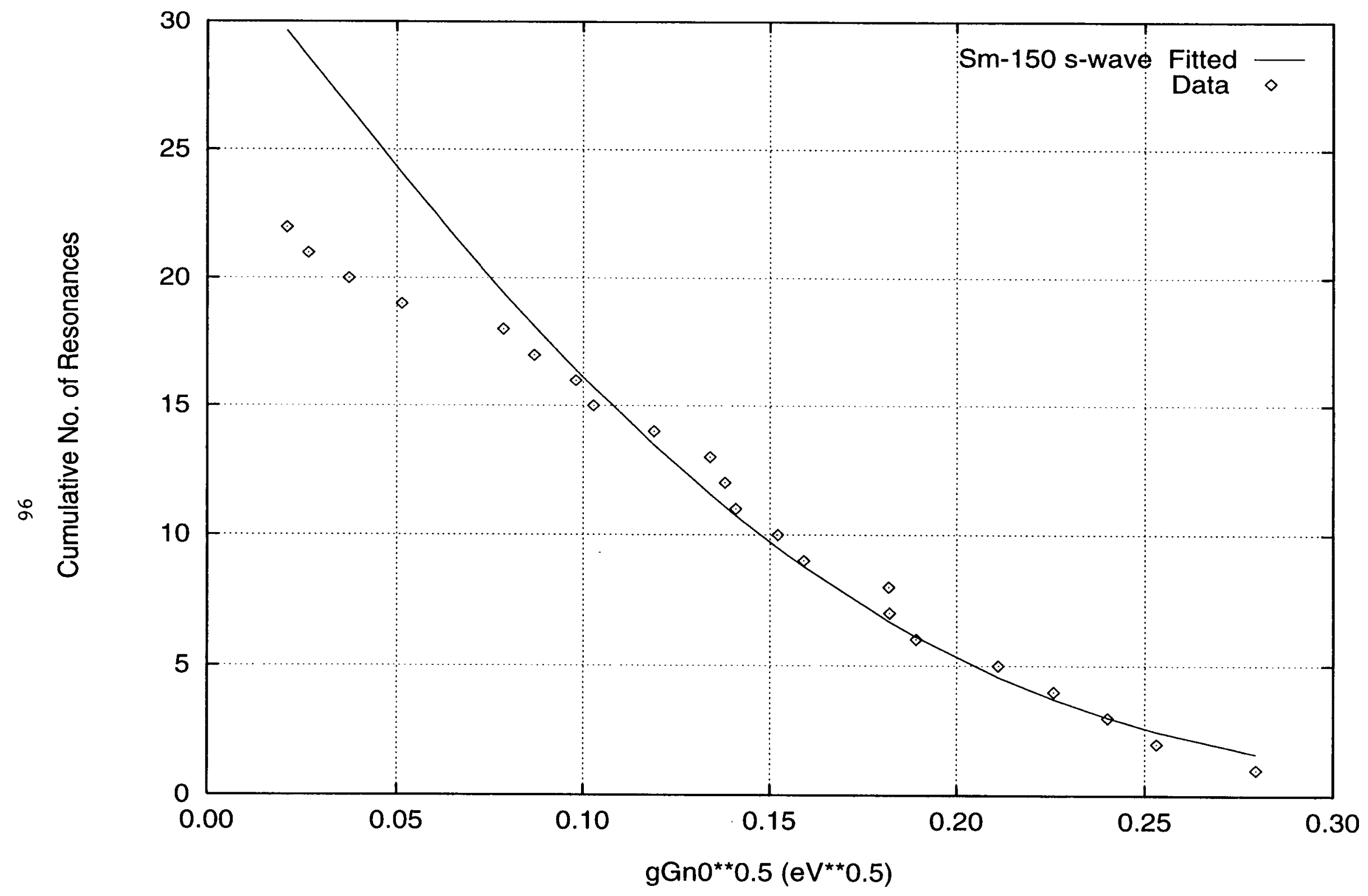

Fig. 43. Complement of the Cumulative Distribution of Neutron Reduced Widths ( ${ }^{150} \mathrm{Sm}, \mathrm{s}$-wave) 


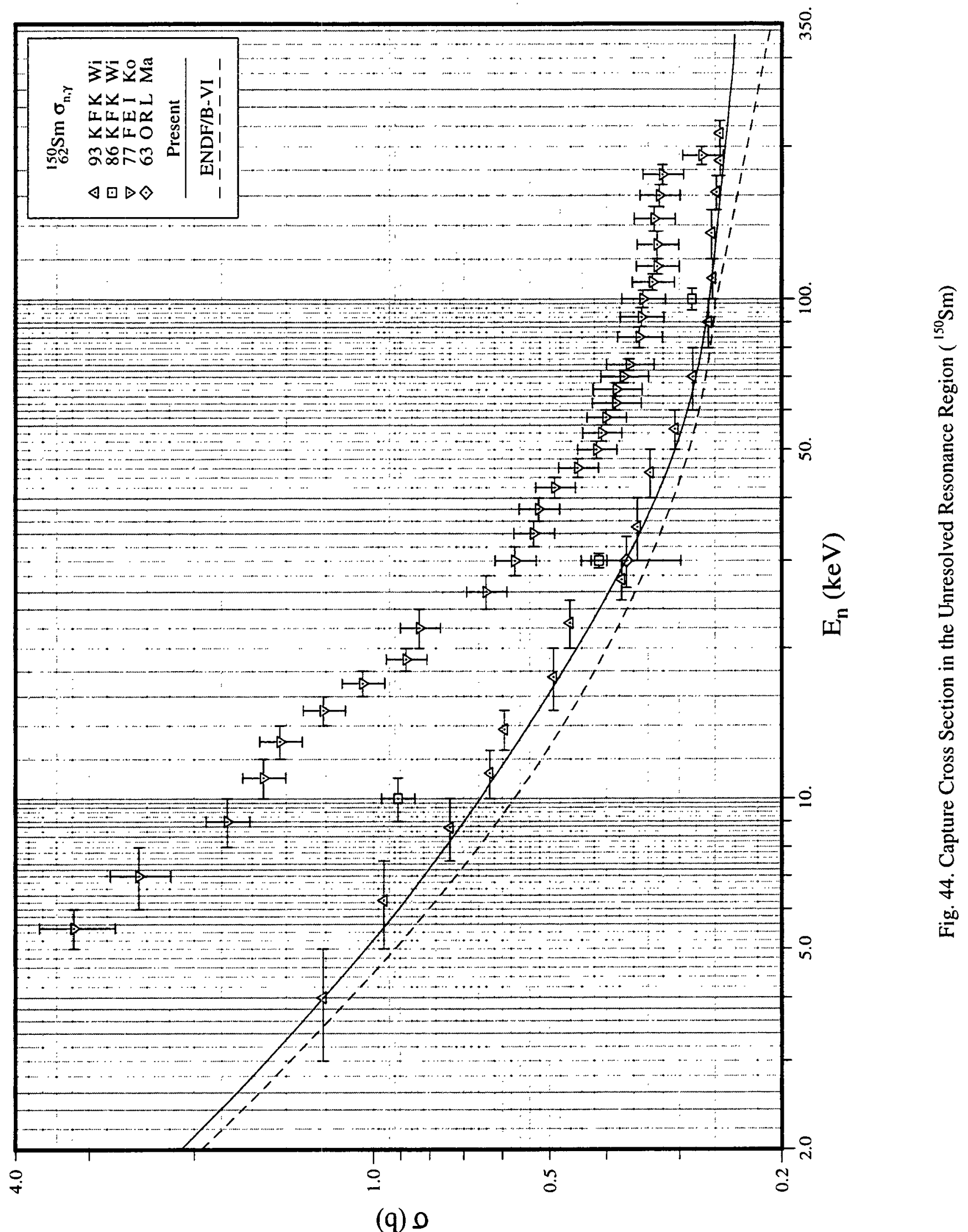




\section{III.O. ${ }^{151} \mathrm{Sm}$}

\section{Thermal Characteristics}

Since the contribution of positive energy resonances [Mu84] to the thermal capture cross section is $235 \mathrm{~b}$, a bound level was stipulated to reproduce a thermal capture cross section $\left(\sigma_{\gamma}{ }^{0}\right)$ of $15200 \pm 300 \mathrm{~b}$ [Mu84]. The resonance parameters of the bound level were determined as: $\mathrm{E}_{0}=-0.12 \mathrm{eV}, \mathrm{J}=2(l=0), \Gamma_{\mathrm{n}}=0.752 \mathrm{meV}$, and $\Gamma_{\gamma}=92.9 \mathrm{meV}$. The effective scattering radius of $8.3 \mathrm{fm}$ was adopted from a deformed optical model calculation [Mu84]. A comparison of the thermal cross sections and resonance integrals of the present evaluation with those of ENDF/BVI, JEF-2 and JENDL-3 evaluations is presented in Table 34. While there is general agreement in the various evaluations of the thermal capture cross sections, as well as the capture resonance integrals, large discrepancies exist in the value of the thermal scattering cross section. Because the scattering cross section is only $0.5 \%$ of the capture cross section, scattering measurements in the thermal region are not available. The calculated Westcott factor, 0.9274 , shows that this nuclide is a non- $1 / \mathrm{v}$ absorber.

\section{Resolved Resonance Parameters}

Because of the unavailability of measurements since 1984, the present evaluation adopted the resolved resonance parameters of the BNL compilation [Mu84]. The resolved energy region extends to $296 \mathrm{eV}$. On the basis of Bayesian analysis, all 120 resonances were assigned as swave resonances. All the resonance spins were assigned randomly on the assumption that the level density is proportional to $(2 \mathrm{~J}+1)$. The uncertainty-weighted average for 13 radiative widths resulted in a value of $92.9 \pm 3.7 \mathrm{meV}$. This value was assumed for the remaining resonances, as well as the bound level. The present average value is in very good agreement with the values $(92 \pm 7 \mathrm{meV})$ [Mu84] and (95 $\pm 4 \mathrm{meV})$ [Re98].

The distribution of s-wave reduced neutron widths was analyzed in terms of the PorterThomas-distribution. Resonances with reduced widths smaller than $0.3 \mathrm{meV}$ were excluded in the fitting procedure (Fig. 45). The fit resulted in $\left\langle\mathrm{g} \Gamma_{\mathrm{n}}{ }^{0}\right\rangle=0.60 \pm 0.08 \mathrm{meV}$ and $\left\langle\mathrm{D}_{0}\right\rangle=1.48 \pm 0.09$ eV. These values yield s-wave strength function of $S_{0}=4.06 \pm 0.56$. The present $\left\langle D_{0}\right\rangle$ is consistent, within the associated uncertainty, with $(1.2 \pm 0.2 \mathrm{eV})$ [Mu84]. However, it deviates from a recommended value of $1.04 \pm 0.15 \mathrm{eV}$ [Re98]. The present $\mathrm{S}_{0}$ is consistent with values in

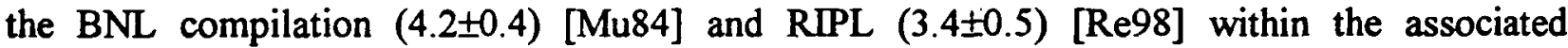
uncertainties.

\section{Unresolved Resonance Parameters}

The unresolved resonance region covers the energy range from $296 \mathrm{eV}$ to $66.24 \mathrm{keV}$. The latter energy corresponds to neutron inelastic scattering to the second excited level of ${ }^{151} \mathrm{Sm}$ at $65.83 \mathrm{keV}$. The energy of the first excited level at $4.82 \mathrm{keV}$ is very low when compared with other fission products. However, the inelastic neutron scattering cross section was not taken into account as a competition channel in $M F=2$ in the present evaluation. Table 35 presents the various evaluations of the capture and inelastic scattering cross sections at $10 \mathrm{keV}$, where the inelastic scattering cross section to the first excited level shows a peak. As indicated, 
disagreements exist in the various evaluations because of differences in the nuclear model calculations, as well as the model parameters.

For this nuclide, s-, $p$ - and d-wave average resonance parameters were provided. The level spacing varies with energy according to Gilbert-Cameron level density formula with associated parameters adopted from Mughabghab and Dunford [Mu98] (neutron separation energy $=8.258 \mathrm{MeV}$, pairing energy $=2.32 \mathrm{MeV}$, and level density parameter $=21.43 \mathrm{MeV}^{-1}$ ). The dependence of the level spacing on spin by the relation $(2 \mathrm{~J}+1) \exp \left\{-(\mathrm{J}+1 / 2)^{2} / 2 \sigma^{2}\right\}$ was taken into account in this evaluation. With a value of $\sigma=3.7$ for the spin dispersion parameter, level spacings for $p$ - and d-wave resonances were calculated as $1 / 1.84$ and $1 / 2.38$ of the s-wave spacing, respectively.

In the unresolved resonance region, capture cross section measurements are not reported. The adopted average resonance parameters are listed in the last column of Table 36. The ENDF/B-VI evaluation does not include unresolved resonance parameter file. The values of $\left\langle\Gamma_{\gamma 0}\right\rangle,\left\langle D_{0}\right\rangle$ and $S_{0}$ are those which resulted from the analysis of the resolved energy region. The present values of $S_{1}$ and $S_{2}$ were adopted from the systematics of strength functions near this mass region [Mu84, Figs. 4 and 5], while the value of $\left\langle\Gamma_{\gamma 1}\right\rangle$ was assumed from systematics of pwave radiative widths [Mu84, Fig. 7]. These parameters resulted in a computed capture cross section that is represented by a solid line in Fig. 46. The present calculation is compared with existing evaluations, as well as a recent calculation of Toukan et al. [To95], represented by diamonds. Our results are in reasonable agreement with those of the ENDF/B-VI evaluation, but not with the JEF-2 evaluation, and are larger than those of Toukan [To95]. We note that other calculations of Toukan resulted in capture cross sections which are lower by $20 \%$, on the average, than those measured by Wisshak et al. [Wi93] for the four $\mathrm{Sm}$ isotopes, ${ }^{147-150} \mathrm{Sm}$.

Maxwellian average capture cross section for a temperature of $30 \mathrm{keV}$ was calculated as $2569 \mathrm{mb}$. In these calculations, which were extended up to $1 \mathrm{MeV}$, the capture cross section was reconstructed from the present resonance parameters up to $66.2 \mathrm{keV}$ and from the ENDF/B-VI evaluation for the energy region from $66.2 \mathrm{keV}$ to $1 \mathrm{MeV}$. Our calculated Maxwellian average capture cross section is large when compared with $1932 \pm 206 \mathrm{mb}$ [Be92] and $1825 \pm 450 \mathrm{mb}$ [To95]. 
Table 34. Thermal Characteristics $\left({ }^{151} \mathrm{Sm}\right)$

\begin{tabular}{|c|c|c|c|c|c|c|c|c|}
\hline Quantity & Unit & $\begin{array}{c}\text { BNL } \\
\text { compilation }\end{array}$ & $\begin{array}{l}98 \mathrm{CRC} \\
\text { [Ho98] }\end{array}$ & $\begin{array}{l}\text { ENDF/ } \\
\text { B-VI }\end{array}$ & JEF-2 & JENDL-3 & $\begin{array}{c}\text { LIPAR-5 } \\
\text { [Ab97] }\end{array}$ & Present \\
\hline $\mathbf{R}^{\prime}$ & $\mathrm{fm}$ & & & 8.30 & 7.20 & 7.95 & & 8.30 \\
\hline $\begin{array}{l}\sigma_{\gamma}^{0} \\
\sigma_{s}^{0}\end{array}$ & $\begin{array}{l}\text { barn } \\
\text { barn }\end{array}$ & $15200 \pm 300$ & $15200 \pm 300$ & $\begin{array}{c}15250 \\
43.5 \\
\end{array}$ & $\begin{array}{c}15190 \\
39.3 \\
\end{array}$ & $\begin{array}{c}15160 \\
50.4 \\
\end{array}$ & $\begin{array}{c}15160 \\
34.4 \\
\end{array}$ & $\begin{array}{c}15170 \\
62.0 \\
\end{array}$ \\
\hline$g_{w}{ }^{*}$ & & & & 0.9308 & 0.9271 & 0.9278 & 0.9256 & 0.9274 \\
\hline $\begin{array}{c}\text { RI- } \\
\text { capt. }^{* *} \\
\text { RI-total }\end{array}$ & $\begin{array}{l}\text { barn } \\
\text { barn }\end{array}$ & $3520 \pm 160$ & $3520 \pm 60$ & $\begin{array}{l}3449 \\
3726\end{array}$ & $\begin{array}{l}3465 \\
3765\end{array}$ & $\begin{array}{l}3410 \\
3752\end{array}$ & $3397^{+}$ & $\begin{array}{l}3430 \\
3765\end{array}$ \\
\hline
\end{tabular}

* Westcott factor for capture cross section.

** Integrated from $0.5 \mathrm{eV}$ to $100 \mathrm{keV}$ with 1/E spectrum; For present calculation, ENDF/B-VI cross sections were imported for an energy region $66.2 \mathrm{keV}$ to $100 \mathrm{keV}$.

+ Integrated from $0.5 \mathrm{eV}$ to $100 \mathrm{eV}$.

Table 35. Capture and Inelastic Scattering Cross Sections at $10 \mathrm{keV}$ of ${ }^{151} \mathrm{Sm}$

\begin{tabular}{|c|c|c|c|}
\hline & ENDF/B-VI & JEF-2.2 & JENDL-3.2 \\
\hline Capture CS (barn) & 5.28 & 6.40 & 3.80 \\
Inel. Scatt. CS (barn) & 4.96 & 1.53 & 0.56 \\
\hline
\end{tabular}

Table 36. Average Resonance Parameters for the Unresolved Resonance Region $\left({ }^{151} \mathrm{Sm}\right)$

\begin{tabular}{|c|c|c|c|c|c|c|c|}
\hline \multirow{2}{*}{ Quantity } & Unit & \multirow{2}{*}{$\begin{array}{c}\text { BNL } \\
\text { compilation }\end{array}$} & \multirow{2}{*}{$\begin{array}{c}\text { ENDF/ } \\
\text { B-VI }\end{array}$} & JEF-2.2 & JENDL- & \multicolumn{2}{|c|}{ Present } \\
\cline { 6 - 8 } & & & & $3.2^{*}$ & $\begin{array}{c}\text { PT } \\
\text { Analysis }\end{array}$ & Adopted \\
\hline $\mathrm{R}^{\prime}$ & $\mathrm{fm}$ & & & 7.46 & 5.72 & & 8.3 \\
\hline$\left\langle\mathrm{D}_{0}\right\rangle$ & $\mathrm{eV}$ & $1.2 \pm 0.2$ & & 1.20 & 4.85 & $1.48 \pm 0.09$ & $1.48^{* *}$ \\
$\mathrm{~S}_{0}$ & $\times 10^{-4}$ & $4.2 \pm 0.4$ & & 4.5 & 4.20 & $4.06 \pm 0.56$ & 4.06 \\
$\left\langle\Gamma_{\gamma 0}\right\rangle$ & $\mathrm{meV}$ & $92 \pm 7$ & & 96.0 & 92.0 & & 92.9 \\
\hline$\left\langle\mathrm{D}_{1}\right\rangle$ & $\mathrm{eV}$ & & & 0.60 & 2.43 & $14.0 \pm 3.5$ & $0.80^{* *}$ \\
$\mathrm{~S}_{1}$ & $\times 10^{-4}$ & & & 1.0 & 1.40 & $1.25 \pm 0.37$ & 0.80 \\
$\left\langle\Gamma_{\gamma 1}\right\rangle$ & $\mathrm{meV}$ & & & $94 \sim 95$ & 92.0 & & 40.0 \\
\hline$\left\langle\mathrm{D}_{2}\right\rangle$ & $\mathrm{eV}$ & & & 0.40 & 1.62 & & $0.62^{* *}$ \\
$\mathrm{~S}_{2}$ & $\times 10^{-4}$ & & & 4.0 & 2.30 & & 2.5 \\
$\left\langle\Gamma_{\gamma 2}\right\rangle$ & $\mathrm{meV}$ & & & $95 \sim 100$ & 92.0 & & 92.9 \\
\hline
\end{tabular}

* Average parameters at the low energy bound of the unresolved resonance region $(0.25 \mathrm{keV})$.

${ }^{* *}$ At the neutron separation energy of ${ }^{152} \mathrm{Sm}$. 


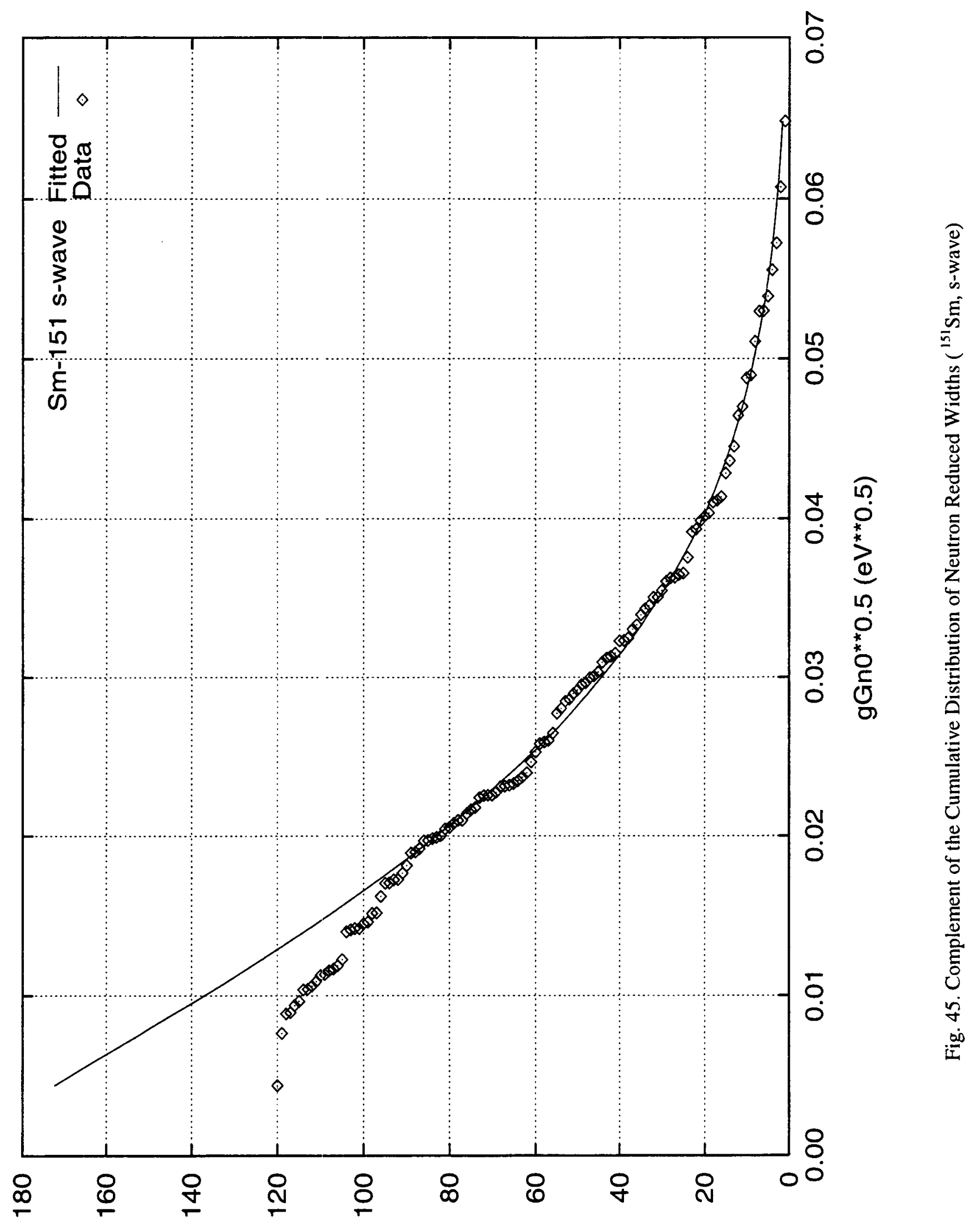

sasueuosəy to 'oN ə^!̣e|nuñ 


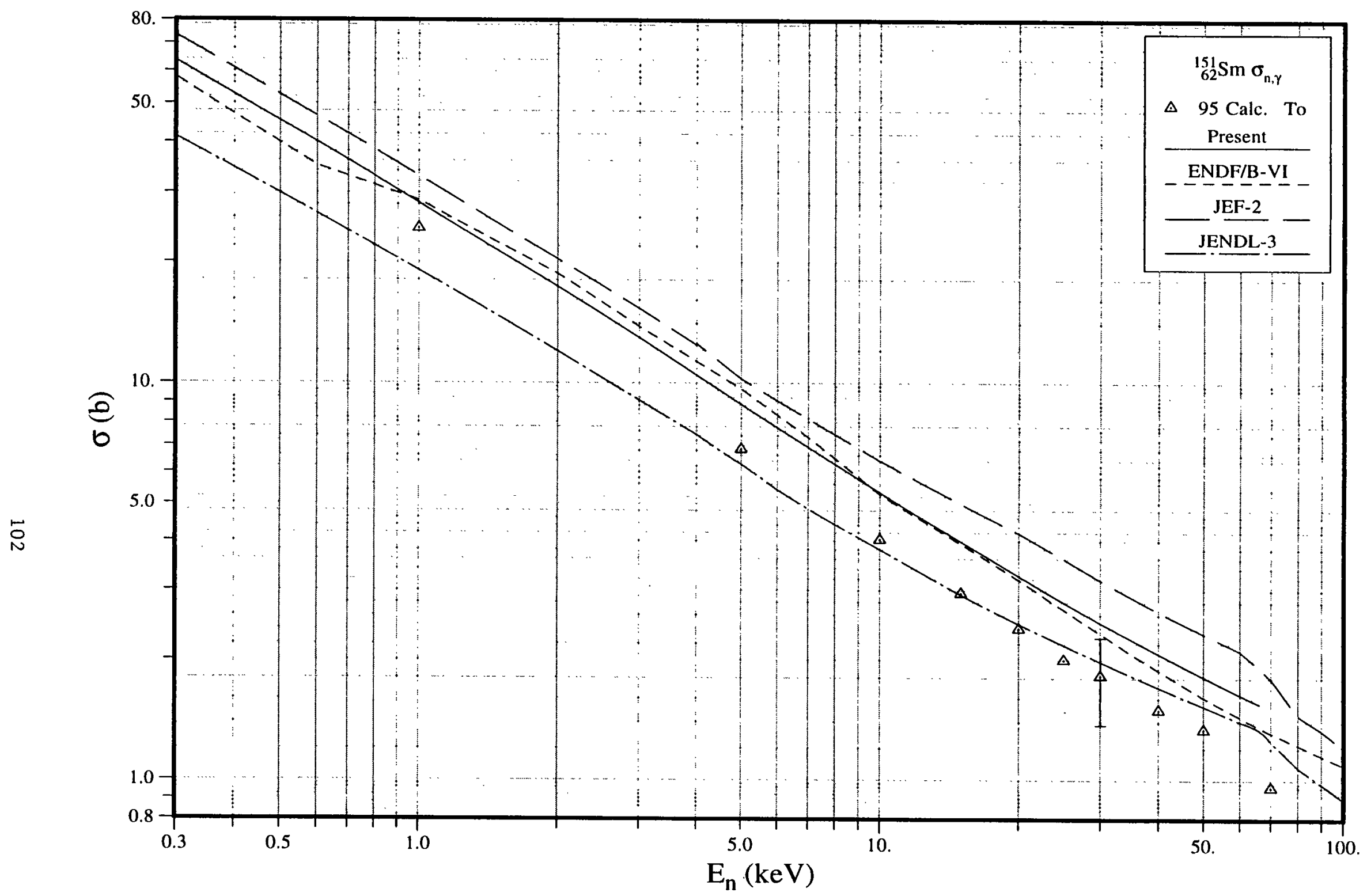

Fig. 46. Capture Cross Section in the Unresolved Resonance Region $\left({ }^{151} \mathrm{Sm}\right)$ 
III.P. ${ }^{152} \mathrm{Sm}$

1. Thermal Region

The resolved resonance parameters, including those of a bound level, and an effective scattering radius R' of $8.3 \mathrm{fm}$ were adopted from the BNL compilation [Mu84]. The contribution of the bound level to the thermal capture cross section at $0.0253 \mathrm{eV}$ is only 2.1 barns. The coherent scattering amplitude of $-5.0 \mathrm{fm}$ [Mu84] is also accounted for by the bound level. As shown in Table 37, the present values are essentially the same as those in the BNL compilation [Mu84] and are in good agreement with those in the ENDF/B-VI and JEF-2.2 valuations. There is disagreement between our calculated scattering cross section and resonance integrals and those of the JENDEL-3.2 evaluation.

\section{Resolved Resonance Parameters}

Because new measurements in the resolved energy region were not carried out since 1984, the resonance parameters reported in the BNL compilation [Mu84] were adopted without modification.

All 91 reported resonances [Mu84] were assigned as s-wave on the basis of the Bayesian analysis. The weighted-average value of nine determined radiative widths resulted in a $\left\langle\Gamma_{\gamma 0}\right\rangle=60.0 \pm 5.4 \mathrm{meV}$. This value is in very good agreement with that presented in the BNL compilation, $61 \pm 7 \mathrm{meV}$ [Mu84]. This average was assumed for the remaining resonances.

The distribution of reduced neutron widths was analyzed by fitting the widths to the Porter-Thomas distribution. As shown in Fig. 47, none of the resonances was excluded from the fitting procedure. The analysis resulted in $\left\langle\mathrm{D}_{0}\right\rangle=47.5 \pm 2.7 \mathrm{eV}$ and $\left\langle\mathrm{g} \Gamma_{\mathrm{n}}{ }^{0}\right\rangle=10.6 \pm 1.6 \mathrm{meV}$. These values yield a strength function for s-wave resonances of $(2.23 \pm 0.35)$, which is in very good agreement with (2.2 \pm 0.3$)$ reported in the BNL compilation [Mu84]. The present $\left\langle\mathrm{D}_{0}\right\rangle$ is also consistent, within the associated uncertainty, with $51.8 \pm 3.3 \mathrm{eV}$ [Mu84]. The resolved energy region consists of $92 \mathrm{~s}$-wave resonances up to $5101.2 \mathrm{eV}$.

\section{Unresolved Resonance Parameters}

The unresolved energy region of the present evaluation extends from $5 \mathrm{keV}$ to 122.58 $\mathrm{keV}$, the latter energy corresponding to neutron inelastic scattering to the first excited state of ${ }^{152} \mathrm{Sm}$.

In the energy range 5 to $200 \mathrm{keV}$, the capture cross sections of Luo et al. [Lu94], Wisshak et al. [Wi93] and Bohkovko et al. [Bo85] were taken into account. The measurement of Kononov et al. [Ko78] was disregarded because the capture cross section is consistently larger than recent data (Fig. 48). Earlier measurements, reported in the Neutron Cross Section Curve book [Mc88], were not taken into account, largely because of their inconsistencies with recent measurements. As depicted in Fig. 48, ENDF/B-VI, JEF-2 and JENDL-3 evaluations, are larger than recent data [Lu94, Wi93, Bo85] by about $10 \%$ to $30 \%$ in the energy region from $70 \mathrm{keV}$ to $120 \mathrm{keV}$. 
As a starting point, the s-wave average resonance parameters, $\left\langle D_{0}\right\rangle, S_{0}$ and $\left\langle\Gamma_{\gamma_{0}}\right\rangle$, derived from the resolved energy region, as well as $S_{1}=0.55$ and $S_{2}=2.2$ [Mu84], were adopted in this energy region. These parameters resulted in a calculated capture cross section, which is low by $\sim 30 \%$ and $\sim 10 \%$ at about energies of $5 \mathrm{keV}$ and $120 \mathrm{keV}$ respectively, when compared with measurement [Wi93]. To achieve agreement with the measurements [Lu94, Wi93, Bo85], $\left\langle\mathrm{D}_{0}\right\rangle$ was set to $34.6 \mathrm{eV}$ and $\left\langle\Gamma_{\gamma 0}\right\rangle$ was increased from $61 \mathrm{eV}$ to $72.7 \mathrm{eV}$ to satisfy s-wave gamma strength function of 0.0021 [Bo91]. In addition, $\left\langle\Gamma_{\gamma_{2}}\right\rangle$ was adjusted to reproduce the capture cross section at high energies. The resulting average resonance parameters are listed in Table 38. The calculated capture cross section is shown in Fig. 49.

In the present evaluation, the level spacing varies with energy according to GilbertCameron level density relation. Parameters for the level density, neutron separation energy, pairing energy, and the single level nuclear density, are found in Mughabghab and Dunford [Mu98a, Mu98b]. The average level spacing and reduced widths, listed in the last column, are the values at the neutron separation energy.

Maxwellian-averaged capture cross section for a temperature of $30 \mathrm{keV}$, computed as 460 $\mathrm{mb}$, is to be compared with, $473.2 \pm 4.4 \mathrm{mb}$ [Wi93], $401 \pm 24 \mathrm{mb}$ [Be92], and $445 \pm 25 \mathrm{mb}$ [Bo91].

Table 37. Thermal Characteristics $\left({ }^{152} \mathrm{Sm}\right)$

\begin{tabular}{|c|c|c|c|c|c|c|c|c|}
\hline Quantity & Unit & $\begin{array}{c}\text { BNL } \\
\text { Comp. }\end{array}$ & $\begin{array}{c}\text { 98CRC } \\
\text { [Ho98] }\end{array}$ & $\begin{array}{c}\text { ENDF/ } \\
\text { B-VI }\end{array}$ & JEF-2.2 & $\begin{array}{c}\text { JENDL- } \\
3.2\end{array}$ & $\begin{array}{c}\text { LIPAR-5 } \\
\text { [Ab97] }\end{array}$ & Present \\
\hline $\mathrm{R}^{\prime}$ & Fm & $8.3 \pm 0.2$ & & 8.3 & 8.3 & 8.2 & & 8.3 \\
\hline$\sigma_{\gamma}{ }^{0}$ & barn & $206 \pm 6$ & $206 \pm 15$ & 207 & 206 & 206 & 201.5 & 206 \\
$\sigma_{\mathrm{s}}{ }^{0}$ & barn & $3.0 \pm 0.2$ & & 3.12 & 2.93 & 0.95 & 7.49 & 3.11 \\
\hline gw $^{*}$ & & & & 1.004 & 1.003 & 1.003 & 1.0032 & 1.0035 \\
\hline $\begin{array}{c}\text { RI- } \\
\text { capt.* }\end{array}$ & barn & $2970 \pm 100$ & $3000 \pm 300$ & 2981 & 2977 & 2770 & $2958^{+}$ & 2976 \\
RI-total $^{* *}$ & barn & & & 9055 & 9048 & 8740 & & 9045 \\
\hline
\end{tabular}

* Westcott factor for capture cross section.

** Integrated from $0.5 \mathrm{eV}$ to $100 \mathrm{keV}$ with $1 / \mathrm{E}$ spectrum.

+ Integrated from $0.5 \mathrm{eV}$ to $2.15 \mathrm{keV}$. 
Table 38. Average Resonance Parameters for the Unresolved Resonance Region $\left({ }^{152} \mathrm{Sm}\right)$

\begin{tabular}{|c|c|c|c|c|c|c|c|}
\hline Quantity & Unit & $\begin{array}{c}\text { ENDF/ } \\
\text { B-VI }\end{array}$ & JEF-2.2 & $\begin{array}{c}\text { JENDL- } \\
3.2^{*}\end{array}$ & $\begin{array}{c}\text { BNL } \\
\text { Comp. }\end{array}$ & $\begin{array}{c}\text { PT Dist'n } \\
\text { Analysis }\end{array}$ & Adopted \\
\hline R' & $\mathrm{fm}$ & 7.665 & 6.438 & 7.556 & $8.3 \pm 0.2$ & & 8.3 \\
(URR) & & & & & & & \\
\hline$\left\langle\mathrm{D}_{0}\right\rangle$ & $\mathrm{eV}$ & 28.00 & 52.52 & 33.46 & $51.8 \pm 3.3$ & $47.5 \pm 2.7$ & 34.6 \\
$\mathrm{~S}_{0}$ & $\times 10^{-4}$ & 2.50 & 2.30 & 2.20 & $2.2 \pm 0.3$ & $2.23 \pm 0.35$ & 2.23 \\
$\left\langle\Gamma_{\gamma 0}\right\rangle$ & $\mathrm{meV}$ & 61.0 & 71.82 & 61.0 & $61 \pm 7$ & & 72.7 \\
\hline$\left\langle\mathrm{D}_{1}\right\rangle$ & $\mathrm{eV}$ & 9.33 & 17.89 & 11.15 & & & 11.5 \\
$\mathrm{~S}_{1}$ & $\times 10^{-4}$ & 0.60 & 1.60 & 0.55 & $0.55 \pm 0.08$ & & 0.60 \\
$\left\langle\Gamma_{\gamma 1}\right\rangle$ & $\mathrm{meV}$ & 61.0 & 71.82 & 61.0 & & & 61.0 \\
\hline$\left\langle\mathrm{D}_{2}\right\rangle$ & $\mathrm{eV}$ & 5.54 & 11.22 & 6.69 & & & 6.91 \\
$\mathrm{~S}_{2}$, & $\times 10^{-4}$ & 3.00 & 6.80 & 2.30 & & & 1.50 \\
$\left\langle\Gamma_{\gamma 2}\right\rangle$ & $\mathrm{meV}$ & 61.0 & 71.82 & 61.0 & & & 34.0 \\
\hline
\end{tabular}

* Average parameters at the low energy $(5.029 \mathrm{keV})$ of the unresolved region. 


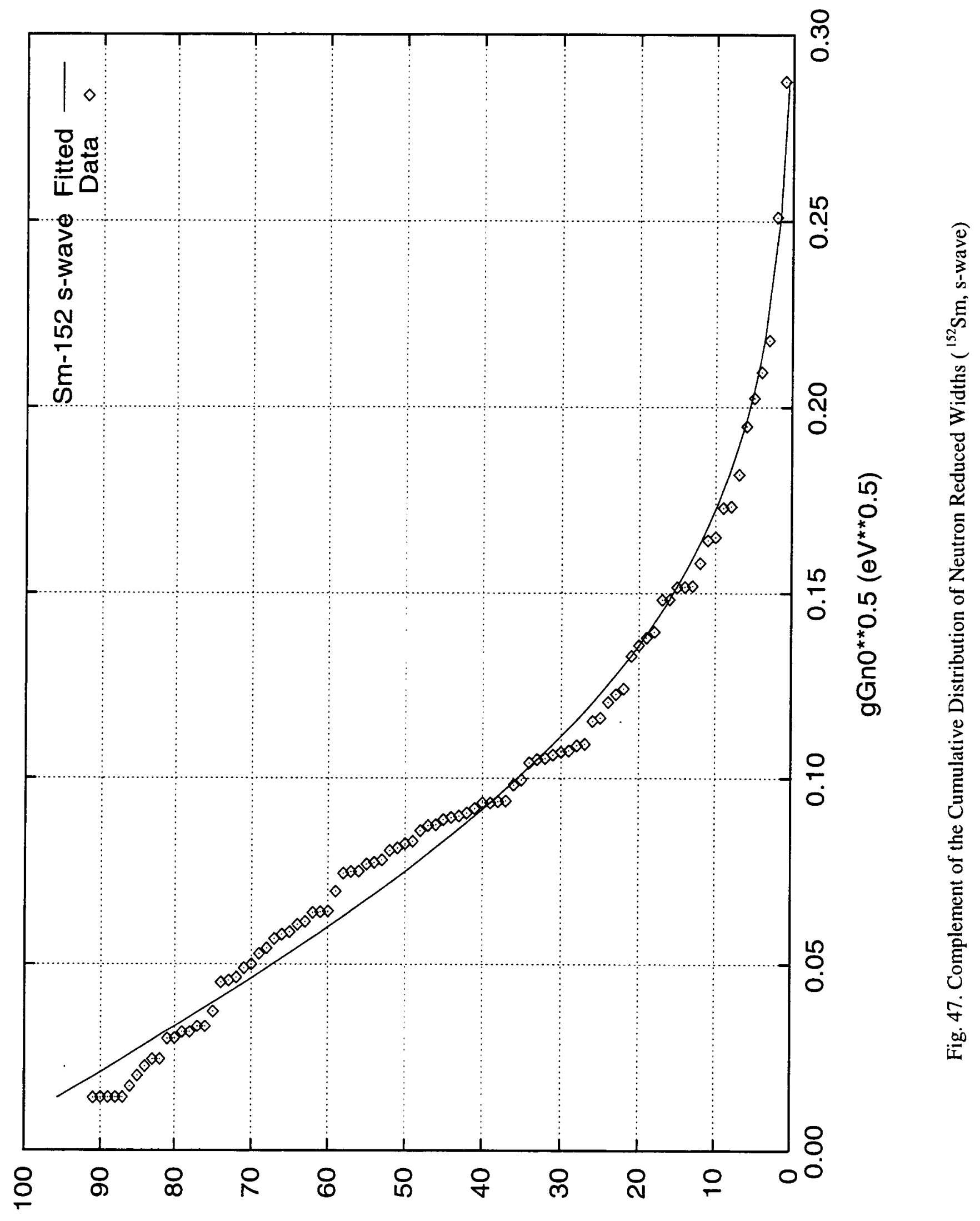

səoueuosəy to 'ON әィ!̣e|nunว 


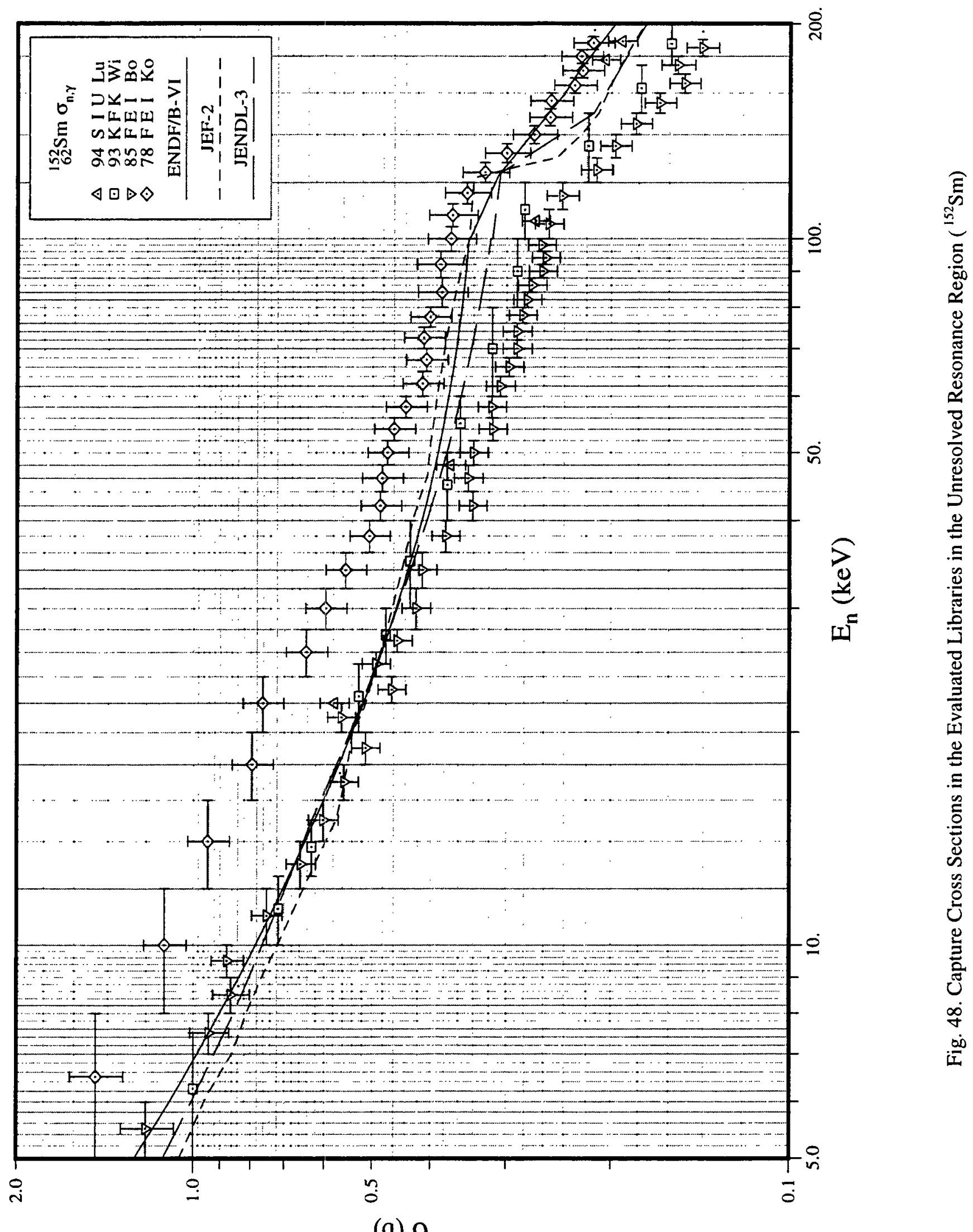




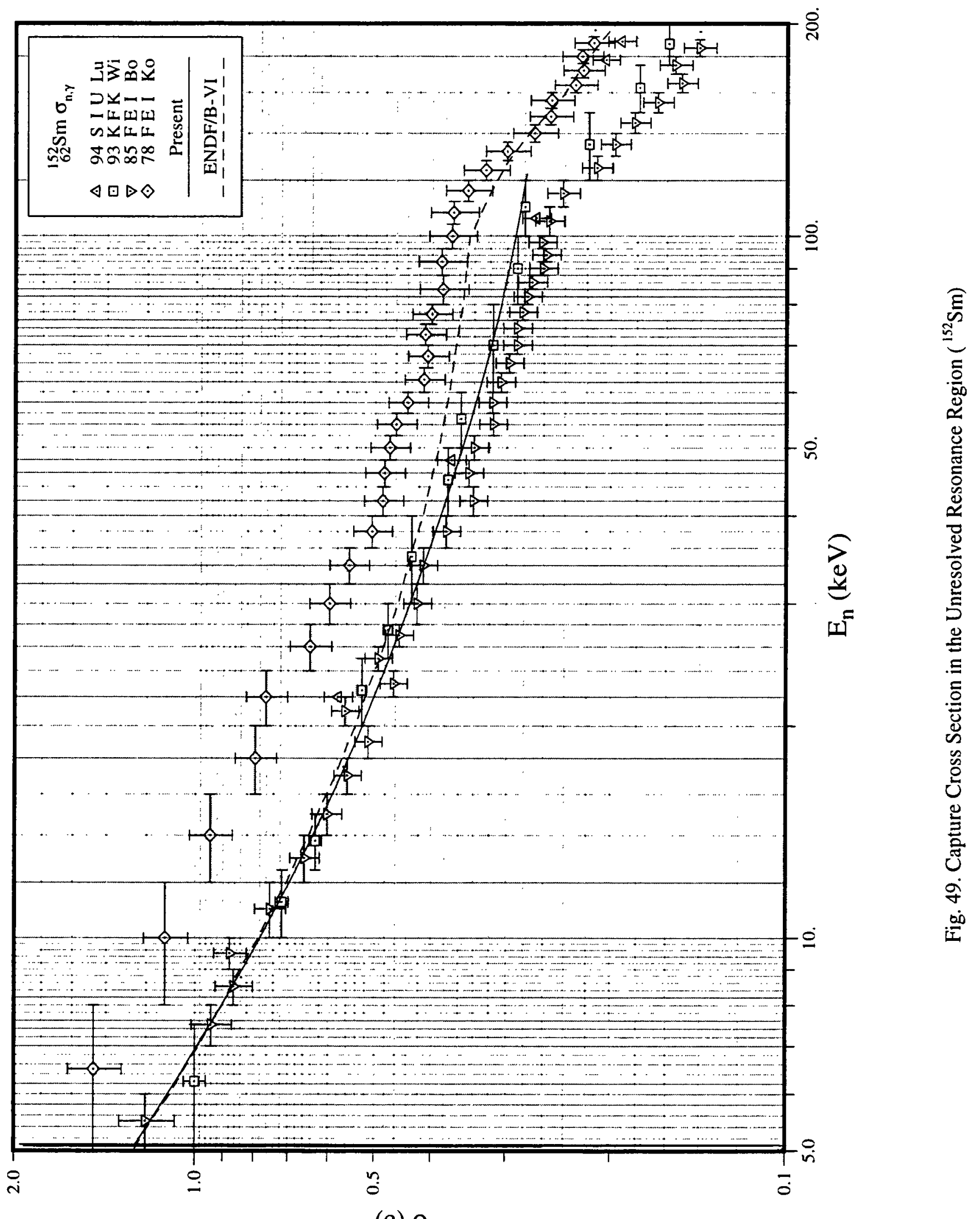

(q) $D$ 


\section{III.Q. ${ }^{153} \mathbf{E u}$}

\section{Thermal Characteristics}

The resolved resonance parameters, including those of a bound level, and the effective scattering radius $R^{\prime}(8.2 \mathrm{fm})$ were adopted from Mughabghab's BNL compilation [Mu84]. The calculated capture cross section at $0.0253 \mathrm{eV}$ due to the bound level is 256.8 barn. As shown in Table 39, the present values are in good agreement with those reported in the BNL compilation [Mu84], as well as JENDL-3.2 evaluation. The present resonance integrals are significantly lower than those in the ENDF/B-VI.5 and JEF-2.2 ${ }^{1}$ evaluations.

\section{Resolved Resonance Parameters}

The resonance parameters up to an energy of $97.8 \mathrm{eV}$ were adopted from the BNL compilation [Mu84].

On the basis of Bayesian analysis, all of the 71 resonances are assigned as s-wave. Note that the s-wave strength function shows a peak while the p-wave strength function has a minimum around this mass region. Only the resonance at $2.456 \mathrm{eV}$ has a spin. Resonance spins were assigned randomly to all the other resonances on the basis of the $(2 \mathrm{~J}+1)$ dependence of the level density. The weighted-average value for the known 55 radiative widths resulted in $\left\langle\Gamma_{\gamma_{0}}\right\rangle=$ $93 \pm 2 \mathrm{meV}$, which was assumed for the remaining resonances.

The distribution of reduced neutron widths was analyzed by fitting to the Porter-Thomas distribution. Three resonances for which $\mathrm{g}_{\mathrm{n}}^{0}$ is smaller than $0.015 \mathrm{meV}$ were excluded from the fitting procedure. Figure 50 shows the cumulative number of resonances for which the widths are greater than the value given on the $\mathrm{x}$-axis versus $\operatorname{Vg} \Gamma_{\mathrm{n}}{ }^{0}$. The fit resulted in $\left\langle\mathrm{D}_{0}\right\rangle=1.14 \pm 0.08$ $\mathrm{eV}$ and $\left\langle\mathrm{g} \Gamma_{\mathrm{n}}{ }^{0}\right\rangle=0.27 \pm 0.05 \mathrm{meV}$. These numbers yield s-wave strength function of $S_{0}=2.37 \pm 0.43$. The present $\left\langle D_{0}\right\rangle$ and $S_{0}$ are consistent, within the uncertainty ranges, with those reported in the BNL compilation [Mu84] $\left(\left\langle\mathrm{D}_{0}\right\rangle=1.3 \pm 0.2 \mathrm{eV}\right.$ and $\left.\mathrm{S}_{0}=2.5 \pm 0.2\right)$ and RIPL [Re98] $\left(\left\langle\mathrm{D}_{0}\right\rangle=1.1 \pm 0.2 \mathrm{eV}\right.$ and $\left.\mathrm{S}_{0}=2.2 \pm 0.3\right)$.

\section{Unresolved Resonance Parameters}

The unresolved resonance region covers the energy region from $97.8 \mathrm{eV}$ to $83.91 \mathrm{keV}$. The upper energy range corresponds to the threshold energy of neutron inelastic scattering to the first excited level of ${ }^{153} \mathrm{Eu}$ at $83.37 \mathrm{keV}$. In the unresolved region, the average resonance parameters for $s-, p$ - and $d$-wave resonances were provided. Since the upper energy of this region is relatively high, $d$-wave resonance contribution to the capture cross section is not negligible. We adopted a level spacing varying with the energy according to the GilbertCameron's level density relation. Parameters for the formula, neutron separation energy, pairing energy and level density, are found in Mughabghab and Dunford [Mu98a Mu98b]. From the

1 Both ENDF/ B-VI and JEF-2 contain the same resonance data, which had been imported from ENDF/B-V. However, since they have different background cross sections in File 3, the resulting thermal cross sections are different from each other. 
$(2 \mathrm{~J}+1)$ dependence of the level density, level spacings for $\mathrm{p}$ - and $\mathrm{d}$-waves were assumed to be $1 / 2$ and $1 / 3$ of the s-wave spacing, respectively.

Capture cross sections measured by Xia et al. [Xi94], Yu et al. [Yu93], Macklin and Young [Ma87], and Bokhovko et al. [Bo85], as well as earlier measurements were taken into account. The cross sections of Mizumoto et al. [Mi79], Kononov et al. [Ko77] and Hockenbury et al. [Ho75] are consistently higher than recent measurements in the energy range of 5 100 $\mathrm{keV}$. The values of $\left\langle D_{0}\right\rangle, S_{0}$ and $\left\langle\Gamma_{\gamma 0}\right\rangle$, deduced from the resolved resonances, and of $S_{1}$ and $S_{2}$ of the BNL compilation [Mu84] resulted in a calculated capture cross section, which is in good agreement with the data in the $1 \sim 30 \mathrm{keV}$ range, but low by $\sim 15 \%$ around $80 \mathrm{keV}$. Subsequently, the $S_{1}$ was increased to 0.6 to improve the fit at the high energy region. The calculated capture cross section is shown in Fig. 51. The finalized average resonance parameters are listed in the last column of Table 40.

Maxwellian-averaged capture cross section for a temperature of $30 \mathrm{keV}$ was computed as $2.52 \mathrm{barn}$. The integration was performed up to an energy of only $83.9 \mathrm{keV}$; this upper energy cutoff may result in an error of about $12 \%$ [Be92]. The present cross section is to be compared with values of $3.17 \pm 0.3 \mathrm{~b}$ reported in Beer's compilation [Be92] and 2.45 $\pm 0.07 \mathrm{~b}$ [Bo91].

Table 39. Thermal Characteristics $\left({ }^{153} \mathrm{Eu}\right)$

\begin{tabular}{|c|c|c|c|c|c|c|c|c|}
\hline Quantity & Unit & $\begin{array}{c}\text { BNL } \\
\text { Compil. }\end{array}$ & $\begin{array}{l}98 \mathrm{CRC} \\
{[\mathrm{Ho} 08]}\end{array}$ & $\begin{array}{c}\text { ENDF/ } \\
\bar{B}-\overline{V I}\end{array}$ & JEF-2.2 & $\begin{array}{c}\text { JENDL- } \\
3.2 \\
\end{array}$ & $\begin{array}{l}\text { LIPAR-5 } \\
\text { [Áb97] }\end{array}$ & Present \\
\hline $\mathbf{R}^{\prime}$ & $\mathrm{fm}$ & $8.2 \pm 0.2$ & & 8.8 & 8.8 & 8.63 & & 8.20 \\
\hline $\begin{array}{l}\sigma_{\gamma}^{0} \\
\sigma_{s}^{0}\end{array}$ & $\begin{array}{l}\text { barn } \\
\text { barn }\end{array}$ & $\begin{array}{c}312 \pm 7 \\
9.7 \pm 0.7\end{array}$ & $300 \pm 20$ & $\begin{array}{l}313 \\
9.04\end{array}$ & $\begin{array}{l}300 \\
6.75\end{array}$ & $\begin{array}{l}313 \\
10.3\end{array}$ & $\begin{array}{l}312 \\
7.68\end{array}$ & $\begin{array}{l}312 \\
9.00\end{array}$ \\
\hline $\mathrm{gw}^{*}$ & & 0.9663 & & 0.9662 & 0.9662 & 0.9869 & 0.9801 & 0.9871 \\
\hline $\begin{array}{c}\mathrm{RI}-. . \\
\text { capt. } \\
\text { RI-total }^{*}\end{array}$ & $\begin{array}{l}\text { barn } \\
\text { barn }\end{array}$ & $1420 \pm 100$ & $1800 \pm 400$ & $\begin{array}{l}1499 \\
1699\end{array}$ & $\begin{array}{l}1448 \\
1644\end{array}$ & $\begin{array}{l}1410 \\
1605\end{array}$ & $1305^{+}$ & $\begin{array}{l}1408 \\
1590\end{array}$ \\
\hline
\end{tabular}

* Westcott factor for capture cross section.

** Integrated from $0.5 \mathrm{eV}$ to $83 \mathrm{keV}$ with $1 / \mathrm{E}$ spectrum.

+ Integrated from $0.5 \mathrm{eV}$ to $46.5 \mathrm{eV}$. 
Table 40. Average Resonance Parameters for the Unresolved Resonance Region $\left({ }^{153} \mathrm{Eu}\right)$

\begin{tabular}{|c|c|c|c|c|c|c|c|}
\hline \multirow{2}{*}{ Quantity } & \multirow{2}{*}{ Unit } & BNL & \multirow{2}{*}{$\begin{array}{c}\text { ENDF/ } \\
\text { Compil. }\end{array}$} & B-VI & JEF-2.2 & JENDL- & \multicolumn{2}{|c|}{$3.2^{*}$} & $\begin{array}{c}\text { PT } \\
\text { Analysis }\end{array}$ & Adopted \\
\hline $\mathrm{R}^{\prime}$ & $\mathrm{fm}$ & $8.2 \pm 0.2$ & 8.80 & 8.80 & 6.421 & & 8.20 \\
\hline$\left\langle\mathrm{D}_{0}\right\rangle$ & $\mathrm{eV}$ & $1.3 \pm 0.2$ & 1.30 & 1.30 & 1.661 & $1.14 \pm 0.08$ & $1.14^{* *}$ \\
$\mathrm{~S}_{0}$ & $\times 10^{-4}$ & $2.5 \pm 0.2$ & 2.50 & 2.50 & 2.79 & $2.37 \pm 0.43$ & 2.37 \\
$\left\langle\Gamma_{\gamma 0}\right\rangle$ & $\mathrm{meV}$ & $93 \pm 3$ & 95.82 & 95.82 & 94.0 & & 93.0 \\
\hline$\left\langle\mathrm{D}_{1}\right\rangle$ & $\mathrm{eV}$ & & 0.65 & 0.65 & 0.831 & & $0.57^{* *}$ \\
$\mathrm{~S}_{1}$ & $\times 10^{-4}$ & $0.3 \pm 0.1$ & 0.60 & 0.60 & 1.497 & & 0.60 \\
$\left\langle\Gamma_{\gamma 1}\right\rangle$ & $\mathrm{meV}$ & & 95.82 & 95.82 & 94.0 & & 93.0 \\
\hline$\left\langle\mathrm{D}_{2}\right\rangle$ & $\mathrm{eV}$ & & & & 0.554 & & $0.38^{* *}$ \\
$\mathrm{~S}_{2}$ & $\times 10^{-4}$ & $5.4 \pm 1.2$ & & & 3.116 & & 5.40 \\
$\left\langle\Gamma_{\gamma 2}\right\rangle$ & $\mathrm{meV}$ & & & & 94.0 & & 93.0 \\
\hline
\end{tabular}

* Average parameters at the low energy $(97.2 \mathrm{eV})$ of the unresolved region.

** At the neutron separation energy of ${ }^{154} \mathrm{Eu}$. 


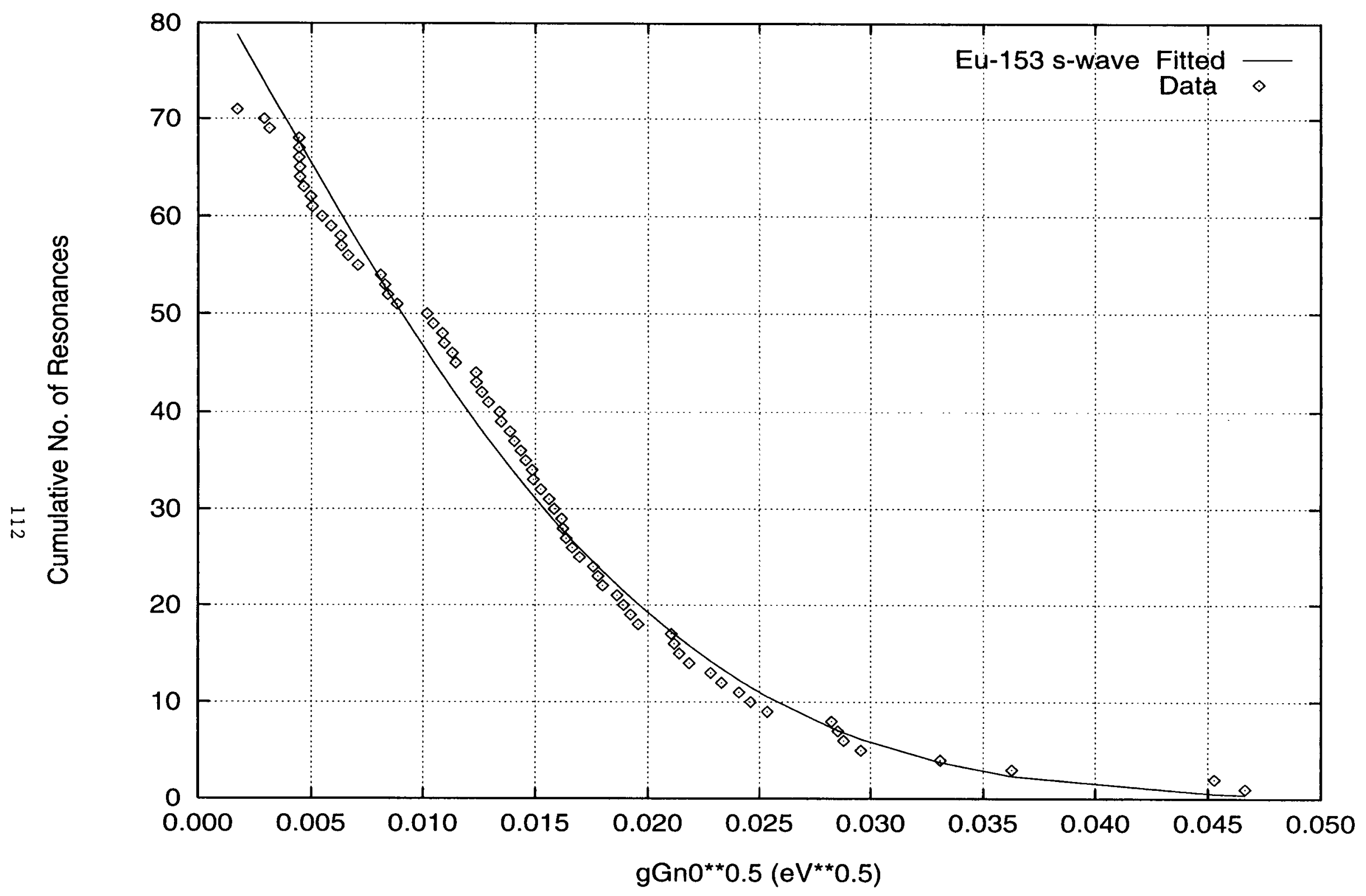

Fig. 50. Complement of the Cumulative Distribution of Neutron Reduced Widths ( ${ }^{153} \mathrm{Eu}$, s-wave) 


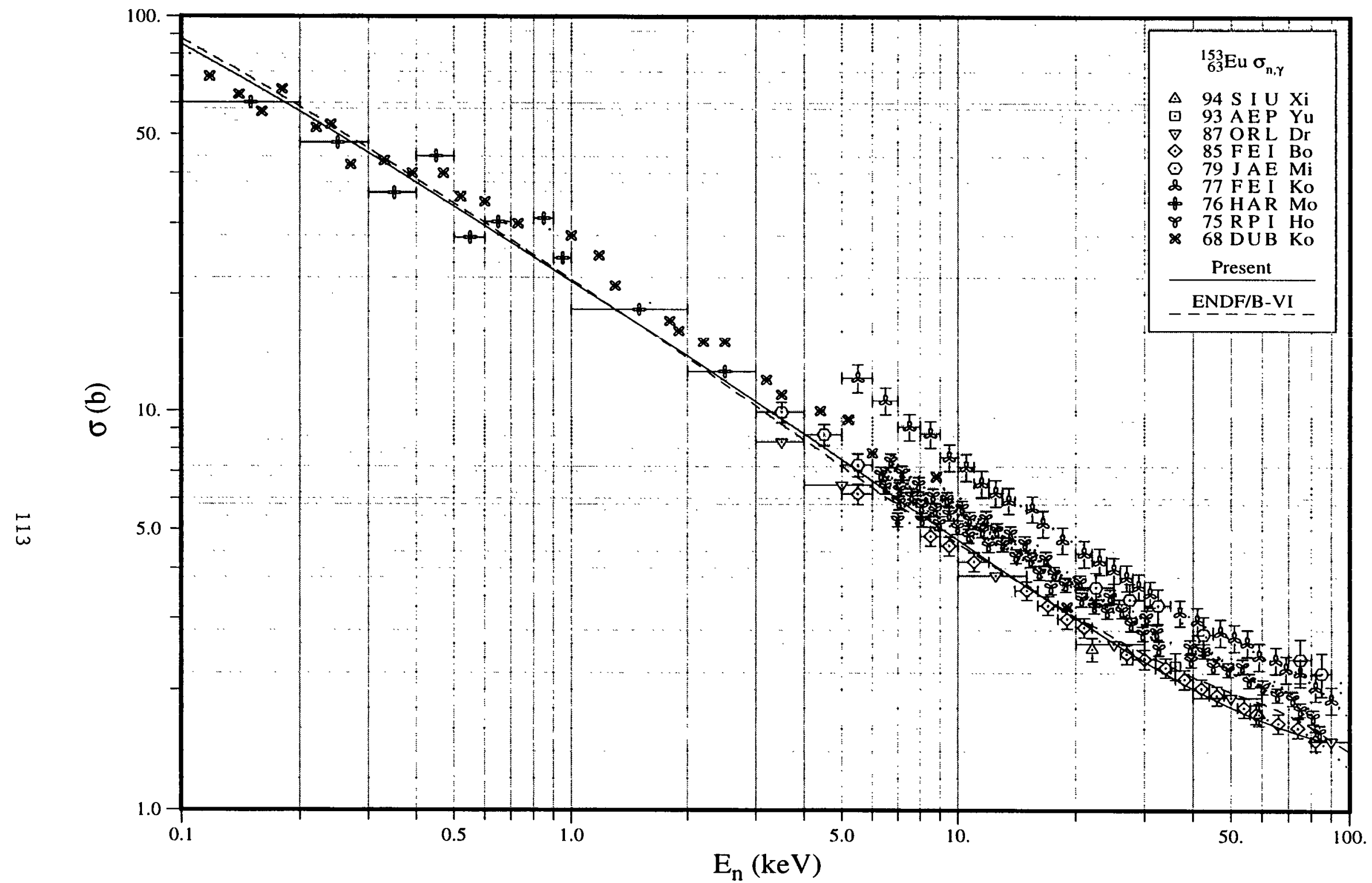

Fig. 51. Capture Cross Section in the Unresolved Resonance Region $\left({ }^{153} \mathrm{Eu}\right)$ 


\section{III.R. ${ }^{155} \mathrm{Gd}$}

\section{Thermal Region}

Within the reported uncertainty of the measured capture cross section, a negative energy resonance is not required to interpret the behavior of the cross section. The calculated thermal cross sections, coherent scattering amplitude, and the capture resonance integral are summarized in Table 41. In these calculations, a potential scattering radius of $8.0 \mathrm{fm}$, based on the systematics [Mu84], was assumed. The calculated Westcott factor for capture is 0.8440 .

Since the first resonance at $0.0268 \mathrm{eV}$ of ${ }^{155} \mathrm{Gd}$ is very close to thermal energy, the scattering, capture, and total cross sections vary significantly with energy. At low energies, the variation of the total cross section multiplied by the square root of the energy, as calculated in the present study, is displayed in Fig. 52 and is compared with Moeller's data [Mo60].

Utilizing R-matrix least-squares fit of the data [Mo60], Lynn and Seeger [Ly90], derived the parameters of the first resonance, which are listed in the third column of Table 42. The calculated energy variation of the real part of the bound coherent scattering length, $\operatorname{Re}\left(b^{\prime}\right)$, [Ly90], designated by open diamonds, is shown in Fig. 53. Applying the Breit-Wigner formalism, we carried out a similar calculation where all positive-energy resonances were included. Our result, represented by a solid line, is displayed in Fig. 53. In our analysis, the parameters of the first positive energy resonance are listed in the last column of Table 42 . As indicated, a large discrepancy exists between our calculation and that of Lynn and Seeger [Ly90]. To understand this disagreement, we carried out several additional calculations. In one calculation, the resonance parameters of the first resonance were changed to those of Lynn and Seeger [Ly90]. Except for a small decrease in the coherent scattering length below $0.04 \mathrm{eV}$, this calculation, indicated by a dotted line, is the same as our former result. In a second calculation in which the effect of the first resonance was taken into account, we obtained the result represented by a dashed line. The calculations are larger than those of [Ly90] throughout the energy region under consideration. In a third calculation, designated by a dash-dotted line, the effect of the second and third resonances at $2.008 \mathrm{eV}$ and $2.568 \mathrm{eV}$ were excluded. For this case, reasonable agreement is achieved with Lynn and Seeger result [Ly90]. The contributions of the second and third resonances to the coherent scattering length are calculated as $+0.12 \mathrm{fm}$ and $+0.63 \mathrm{fm}$, respectively.

\section{Resolved Resonance Parameters}

The resonance parameters of the BNL compilation [Mu84] were adopted in this evaluation. In addition, the resonance spin assignments of Belyaev et al. [Be90], which were made by the gamma multiplicity method in the energy range from 6.3 to $183.3 \mathrm{eV}$, were considered.

The weighted-average radiative width was determined as $114 \pm 3 \mathrm{meV}$ from 61 resonances with reported radiative widths. 
All of the reported resonances were determined as s-wave according to Bayesian analysis. As displayed in Fig. 54, the analysis of reduced neutron widths according to the PorterThomas distribution suggests that many weak s-wave resonances are missed. A least-squares fit to the Porter-Thomas distribution was carried out for values of $\left(g \Gamma_{n}^{0}\right)^{1 / 2}>0.007 \mathrm{eV}$. The average s-wave resonance parameters determined from this analysis are listed in Table 43.

The upper energy of the resolved resonance region was set to $183.4 \mathrm{eV}$.

\section{Unresolved Resonance Parameters}

Since inelastic neutron scattering to the first excited state of ${ }^{155} \mathrm{Gd}$ is at $60.009 \mathrm{keV}$ in the center-of-mass system, the upper energy limit of the unresolved resonance region was set to $60.40 \mathrm{keV}$.

The capture data of Shorin et al. at IPPE [Sh74], Beer and Macklin at ORELA [Be88], Nakajima et al. at JAERI [Na89], and Wisshak et al. at Karlsruhe [Wi95] were considered. As shown in Fig. 55, Shorin's data is higher than the more recent results [Be88, Na89, Wi95].

In the present evaluation, the average s-wave resonance parameters obtained from resolved resonances were adopted without adjustments. The energy-dependent level spacing was assumed to vary with energy and spin according to Gilbert-Cameron level density formula with associated parameters of Mughabghab and Dunford [Mu98a] and a spin dispersion parameter of 3.5 [Mu98b]. Since d-wave contribution is negligible below $60 \mathrm{keV}$, its effect was not included in the calculations. The average $p$-wave parameters, $\left\langle\Gamma_{\gamma 1}>=0.050 \mathrm{eV}, \mathrm{S}_{1}=2.0\right.$, were adopted from the systematics and optical model calculations [Mu84]. As shown in Fig. 55, the calculated capture cross sections supports the measurement of Wisshak et al. [Wi95] and Beer and Macklin [Be88]. Note that the ENDF/B-VI evaluation significantly diverges from the present evaluation below $20 \mathrm{keV}$.

Maxwellian average capture cross section for a temperature of $30 \mathrm{keV}$ was computed as $2564 \mathrm{mb}$. The computation was carried out up to $1 \mathrm{MeV}$. The capture cross section was constructed with the present parameters up to $60.4 \mathrm{keV}$ and from this energy to $1 \mathrm{MeV}$ with that of the ENDF/B-VI evaluation. Our result is to be compared with $2648 \pm 30 \mathrm{mb}$ [Wi95] and $2721 \pm 90$ $\mathrm{mb}[\mathrm{Be} 92]$. 
Table 41. Thermal Characteristics $\left({ }^{155} \mathrm{Gd}\right)$

\begin{tabular}{|c|c|c|c|c|c|c|c|}
\hline Quantity & Unit & $\begin{array}{c}\text { BNL } \\
\text { Compilation }\end{array}$ & $\begin{array}{c}\text { 98CRC } \\
\text { [Ho98] }\end{array}$ & $\begin{array}{c}\text { ENDF/ } \\
\text { B-VI }\end{array}$ & JEF-2.2 & $\begin{array}{c}\text { JENDL- } \\
3.2\end{array}$ & Present \\
\hline$R^{\prime}$ & $\mathrm{fm}$ & & & 6.70 & 6.70 & 6.70 & 8.0 \\
$\operatorname{Re}\left(b^{\prime}\right)^{*}$ & $\mathrm{fm}$ & 13.8 & & 12.74 & & 12.74 & 14.02 \\
\hline$\sigma_{r}$ & barns & $60900 \pm 500$ & $61000 \pm 1000$ & 61100 & 60790 & 60890 & 60730 \\
$\sigma_{s}$ & barns & 60 & & 58.9 & 59.0 & 59.0 & 60.7 \\
$\sigma_{\text {coh }}$ & barns & 38.6 & & & & & 38.6 \\
\hline RI-capt. & barns & $1447 \pm 100$ & $1540 \pm 100$ & 1555 & 1543 & 1540 & 1537 \\
\hline
\end{tabular}

* at $0.08 \mathrm{eV}$.

Table 42. Parameters of the First Resonance and Scattering Lengths (Real Part) of ${ }^{155} \mathrm{Gd}$

\begin{tabular}{|c|c|c|c|}
\hline Parameter & Unit & Lynn & Present \\
\hline Scattering Radius & $\mathrm{fm}$ & $6.831^{*}$ & 8.00 \\
\hline Resonance Energy & $\mathrm{eV}$ & 0.0281 & 0.0268 \\
Neutron Width & $\mathrm{meV}$ & 0.104 & 0.104 \\
Radiative Width & $\mathrm{meV}$ & 105 & 108 \\
\hline Scattering Length at $0.08 \mathrm{eV}$ & $\mathrm{fm}$ & 15.27 & 14.02 \\
\hline
\end{tabular}

* Potential radius for R-matrix formulation. 
Table 43. Average Resonance Parameters in the Unresolved Resonance Region $\left({ }^{155} \mathrm{Gd}\right)$

\begin{tabular}{|c|c|c|c|c|c|c|c|}
\hline \multirow[b]{2}{*}{ Quantity } & \multirow[b]{2}{*}{ Unit } & \multirow{2}{*}{$\begin{array}{c}\text { ENDF/B- } \\
\text { VI }\end{array}$} & \multirow[b]{2}{*}{ JEF-2.2 } & \multirow{2}{*}{$\begin{array}{c}\text { JENDL- } \\
3.2^{2}\end{array}$} & \multirow{2}{*}{$\begin{array}{l}\text { BNL } \\
\text { Compil. }\end{array}$} & \multicolumn{2}{|c|}{ Present } \\
\hline & & & & & & $\begin{array}{c}\text { PT } \\
\text { Analysis }\end{array}$ & Adopted \\
\hline$R^{\prime}$ & $\mathrm{fm}$ & 6.70 & 3.59 & 6.83 & $6.7 \pm 1.5$ & & 8.00 \\
\hline$<D_{0}>$ & $\mathrm{eV}$ & 1.27 & 2.20 & 0.857 & $1.8 \pm 0.2$ & $1.45 \pm 0.09$ & $1.45^{b}$ \\
\hline$S_{0}$ & $\times 10^{-4}$ & 3.08 & 3.00 & 2.00 & $2.0 \pm 0.2$ & $2.13 \pm 0.35$ & 2.13 \\
\hline$<\Gamma_{r 0}>$ & $\mathrm{meV}$ & 112 & 120 & 110 & $110 \pm 3$ & & 114 \\
\hline$\left\langle D_{1}\right\rangle$ & eV & 1.27 & 1.10 & 0.429 & & & $0.81^{b}$ \\
\hline$S_{1}$ & $\times 10^{-4}$ & 0.80 & 3.70 & 1.10 & & & 2.00 \\
\hline$<\Gamma_{\gamma 1}>$ & $\mathrm{meV}$ & 112 & 120 & 110 & & & 50.0 \\
\hline$<D_{2}>$ & $\mathrm{eV}$ & & 0.705 & 0.274 & & & \\
\hline$S_{2}$ & $\times 10^{-4}$ & & 1.0 & 2.30 & & & \\
\hline$<\Gamma_{y^{2}}>$ & $\mathrm{meV}$ & & 120 & 110 & & & \\
\hline
\end{tabular}

a: At the lowest energy $(182 \mathrm{eV})$ of the unresolved resonance region.

b: At the neutron separation energy of ${ }^{156} \mathrm{Gd}$. 


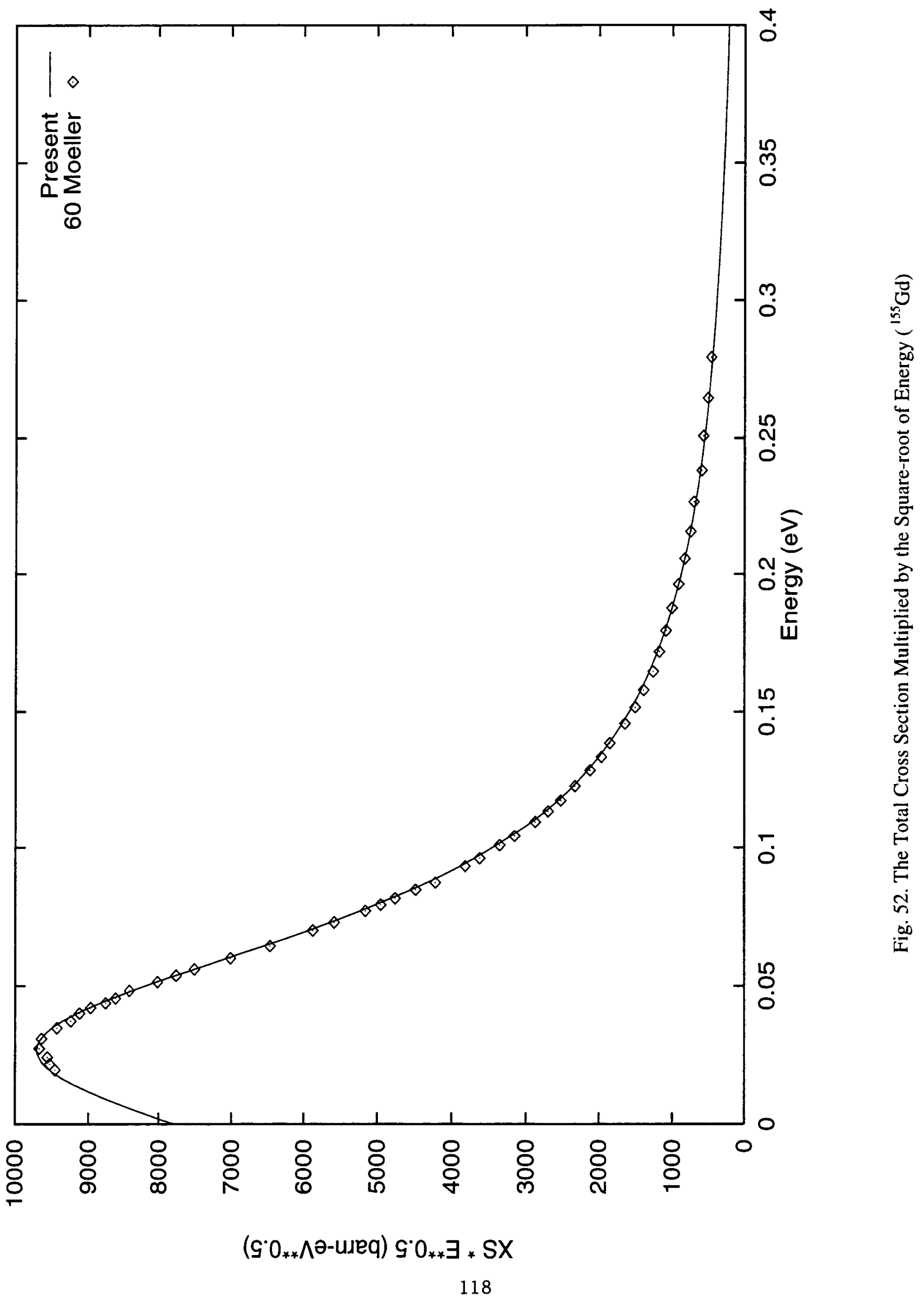




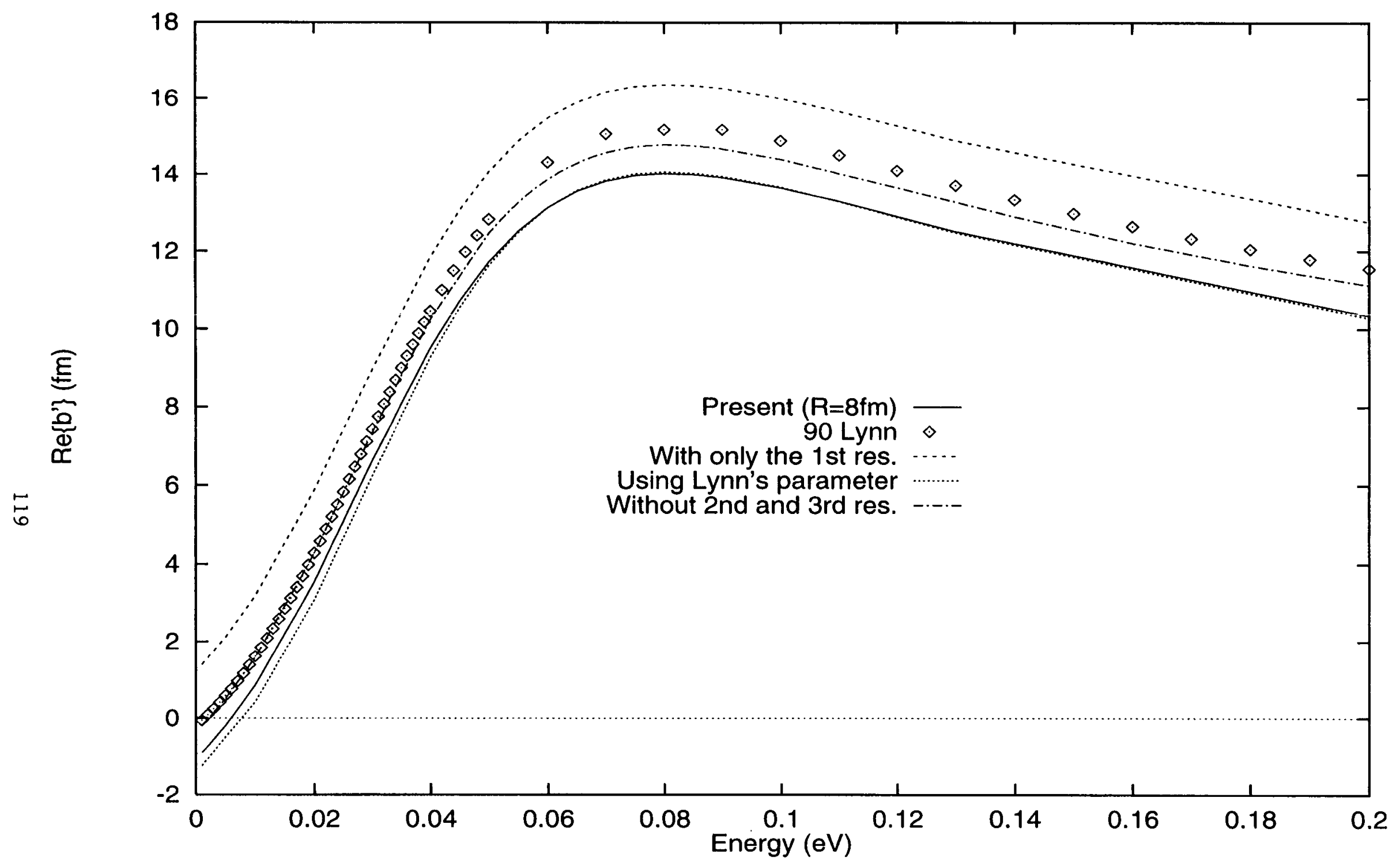

Fig. 53. Real Part of the Bound Coherent Scattering Length of ${ }^{155} \mathrm{Gd}$ 


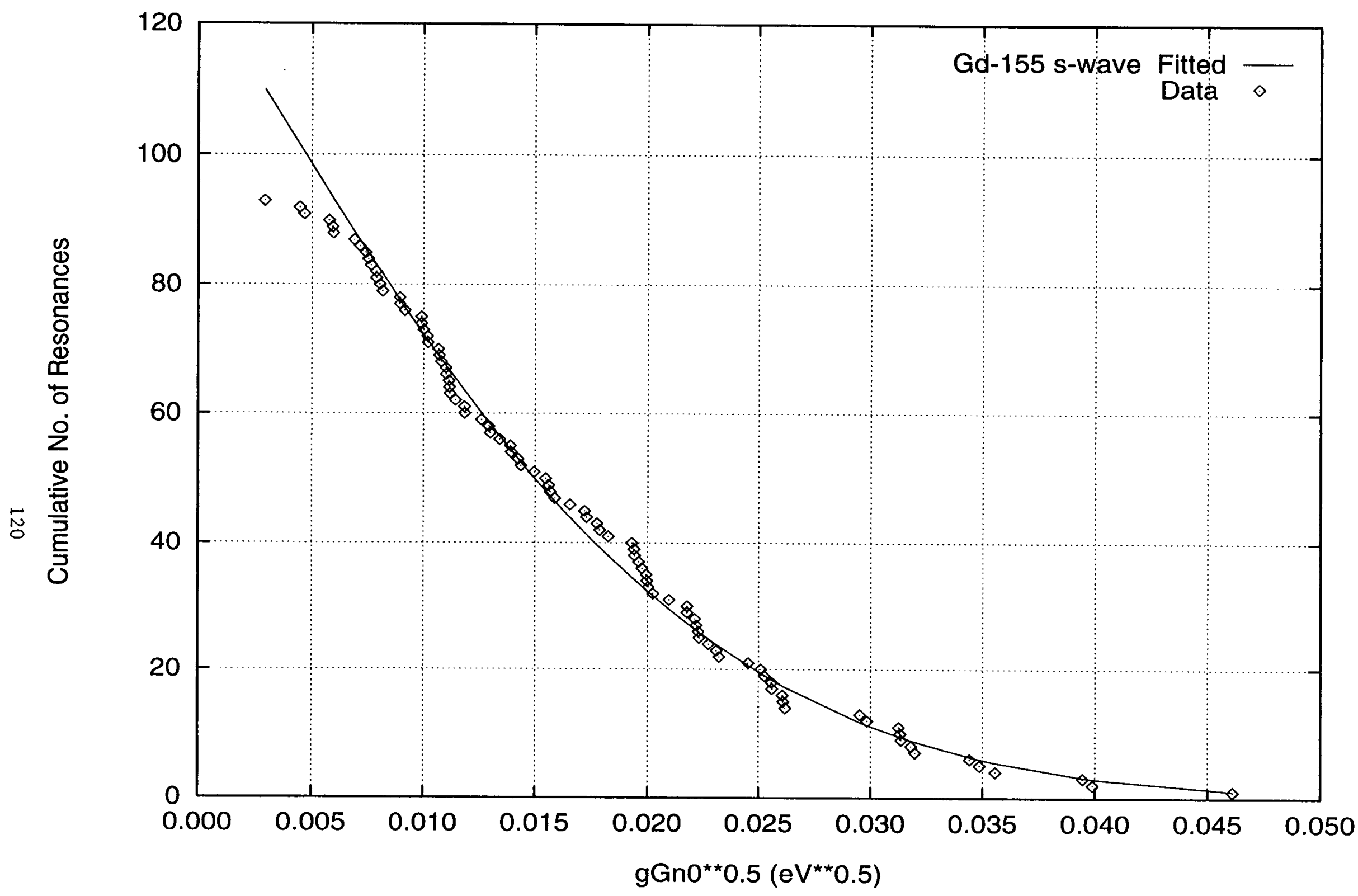

Fig. 54. Complement of the Cumulative Distribution of Neutron Reduced Widths ( ${ }^{155} \mathrm{Gd}$, s-wave) 


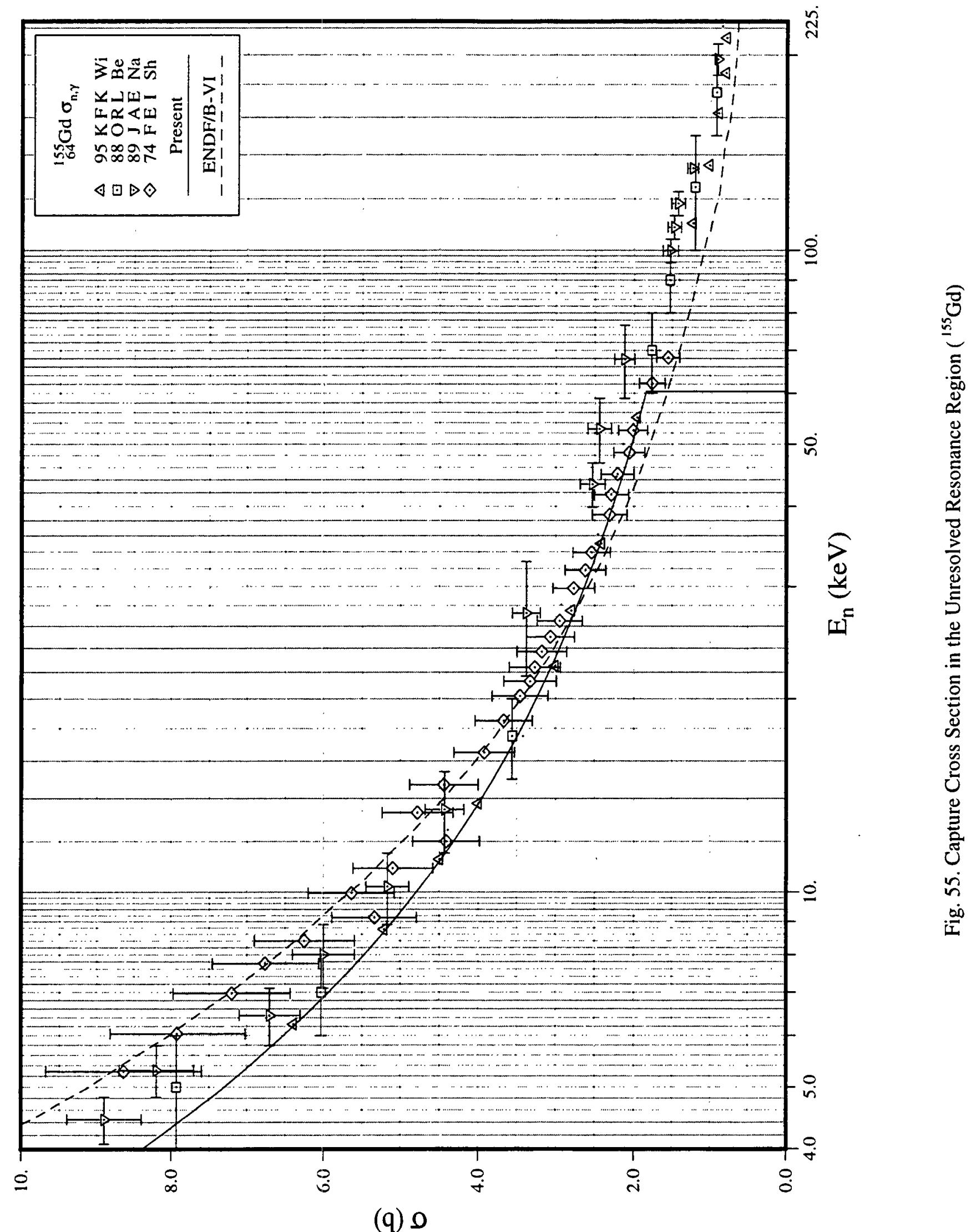




\section{III.S. ${ }^{157} \mathbf{G d}$}

\section{Thermal Region}

The resolved resonance parameters were adopted from Mughabghab's compilation [Mu84]; an effective scattering radius $R^{\prime}$ of $7.8 \mathrm{fm}$, which was read off from a deformed optical model calculation [Mu84], was assumed. For this nuclide, the total contribution of positiveenergy resonances to the thermal capture cross section at $0.0253 \mathrm{eV}$ is calculated as $253.7 \mathrm{~kb}$, which is consistent with the value in the BNL compilation ( $254.0 \pm 0.815 \mathrm{~kb})$. Therefore, a bound level is not required. The thermal characteristics are summarized in Table 44.

Because of the strong resonance at $0.0314 \mathrm{eV}$, the energy dependence of the real and imaginary parts of the coherent scattering lengths was investigated. Figure 56 shows our calculated coherent scattering length, which is based on the present resonance parameters, and is compared with the calculations of Lynn and Seeger [Ly90].

\section{Resolved Resonance Parameters}

The resolved resonance parameters were adopted from the BNL compilation [Mu84]. Spin assignments made by Belyaev et al. [Be90] by the $\gamma$ ray multiplicity method were incorporated into the present evaluation. On the basis of Bayesian analysis, all of the 60 resonances up to an energy of $306.6 \mathrm{eV}$ were assigned as s-wave. Spins of 53 resonances are reported in the BNL compilation. For the remaining resonances, spins were assigned randomly on the basis of the $(2 \mathrm{~J}+1)$ dependence of the level density. Weighted averaging of 27 measured gamma widths resulted in a value of $100 \pm 8 \mathrm{meV}$; this value was assumed for the remaining resonances. The average radiative width is consistent with those reported in the BNL compilation ( $97 \pm 8 \mathrm{meV}$ ) [Mu84], and determined by Nakajima et al. [Na89], $115 \pm 28 \mathrm{meV}$.

The distribution of reduced neutron widths was analyzed in terms of a Porter-Thomas distribution. None of the weak resonances was excluded from the fitting. Figure 57 shows a fit to the data, which resulted in $\left\langle D_{0}\right\rangle=4.48 \pm 0.33 \mathrm{eV},\left\langle\mathrm{g} \Gamma_{\mathrm{n}}{ }^{0}\right\rangle=1.01 \pm 0.19 \mathrm{meV}$, and s-wave strength function of $S_{0}=2.25 \pm 0.44$. The present $\left\langle D_{0}\right\rangle$ is consistent with reported values, (4.9 \pm 0.4$)$ [Mu84] and (4.910.5) [Re98], within the associated uncertainty. The $S_{0}$ value is consistent with

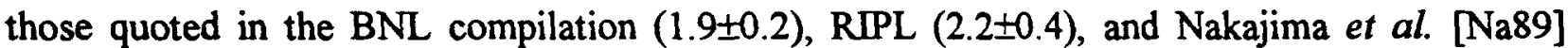
$(2.23 \pm 0.57)$.

\section{Unresolved Resonance Parameters}

The present unresolved region covers the energy range from $306.6 \mathrm{eV}$ to $54.88 \mathrm{keV}$. The latter energy corresponds to the threshold energy for inelastic neutron scattering to the first excited level of ${ }^{15 t} \mathrm{Gd}$ at $54.53 \mathrm{keV}$. For this isotope, s- and p-wave average resonance parameters were provided. Since the upper energy is relatively low, d-wave contribution is negligible. The level spacing varies with energy according to Gilbert-Cameron level density formula with associated parameters adopted from Mughabghab and Dunford [Mu98]. Based on a $(2 \mathrm{~J}+1)$ dependence of the level density, the $\mathrm{p}$-wave level spacing is assumed to be $1 / 2$ of the swave spacing. 
In the unresolved resonance region, the average capture cross sections measured by Wisshak et al. [Wi95], Nakajima et al. [Na89], and Beer and Macklin [Be88] were taken into consideration. These sets show good agreement with each other. The final unresolved resonance parameters, listed in the last column of Table 45, resulted in a calculated capture cross section in agreement with the experimental data (Fig. 58).

Table 44. Thermal Characteristics $\left({ }^{157} \mathrm{Gd}\right)$

\begin{tabular}{|c|c|c|c|c|c|c|c|c|}
\hline Quantity & Unit & $\begin{array}{c}\text { BNL } \\
\text { compil. }\end{array}$ & $\begin{array}{l}98 \mathrm{CRC} \\
{[\mathrm{Ho98}]}\end{array}$ & $\begin{array}{c}\text { ENDF/ } \\
\text { B-VI }\end{array}$ & JEF-2.2 & $\begin{array}{c}\text { JENDL- } \\
3.2\end{array}$ & $\begin{array}{l}\text { LIPAR } \\
\text { [Ab97] }\end{array}$ & Present \\
\hline $\mathbf{R}^{\prime}$ & $\mathrm{fm}$ & & & 4.90 & 4.90 & 4.90 & & 7.80 \\
\hline $\begin{array}{c}\sigma_{\gamma}^{0} \\
\sigma_{s}^{0} \\
\sigma_{\text {cohh }}{ }^{0} \\
\sigma_{\text {incoh }}{ }^{0}\end{array}$ & $\begin{array}{l}\text { kbarn } \\
\text { barn } \\
\text { barn } \\
\text { barn }\end{array}$ & $\begin{array}{c}254 \pm 0.815 \\
1000 \\
622 \\
378\end{array}$ & $254 \pm 3$ & $\begin{array}{l}255.8 \\
1015\end{array}$ & $\begin{array}{l}253.4 \\
1756\end{array}$ & $\begin{array}{c}254.1 \\
1007\end{array}$ & $\begin{array}{l}253.5 \\
1005\end{array}$ & $\begin{array}{c}253.7 \\
1009 \\
627 \\
382\end{array}$ \\
\hline$g_{w}^{*}$ & & & & 0.8500 & 0.8514 & 0.8522 & 0.8527 & 0.8515 \\
\hline $\begin{array}{c}\text { RI- } \\
\text { capt. }^{* *} \\
\text { RI-total }^{* *}\end{array}$ & $\begin{array}{l}\text { barn } \\
\text { barn }\end{array}$ & $700 \pm 20$ & $800 \pm 100$ & $\begin{array}{l}759 \\
960\end{array}$ & $\begin{array}{l}762 \\
946\end{array}$ & $\begin{array}{l}763 \\
936\end{array}$ & $711^{+}$ & $\begin{array}{l}754 \\
989\end{array}$ \\
\hline
\end{tabular}

* Westcott factor for capture cross section.

** Integrated from $0.5 \mathrm{eV}$ to $100 \mathrm{keV}$ with $1 / \mathrm{E}$ spectrum.

+ Integrated from 0.5 to $215 \mathrm{eV}$.

Table 45. Average Resonance Parameters for the Unresolved Resonance Region $\left({ }^{157} \mathrm{Gd}\right)$

\begin{tabular}{|c|c|c|c|l|c|c|c|}
\hline \multirow{2}{*}{ Quantity } & Unit & \multirow{2}{*}{$\begin{array}{c}\text { BNL } \\
\text { compil. }\end{array}$} & \multirow{2}{*}{$\begin{array}{c}\text { ENDF/ } \\
\text { B-VI }\end{array}$} & JEF-2.2 & JENDL- & \multicolumn{2}{|c|}{ Present } \\
\cline { 6 - 8 } & & & & & $\begin{array}{c}\text { PT } \\
\text { Analysis }\end{array}$ & Adopted \\
\hline $\mathrm{R}^{\prime}$ & $\mathrm{fm}$ & & 4.90 & & 6.88 & & 7.80 \\
\hline$\left\langle\mathrm{D}_{0}\right\rangle$ & $\mathrm{eV}$ & $4.9 \pm 0.4$ & 3.53 & & 3.94 & $4.48 \pm 0.33$ & $4.48^{* *}$ \\
$\mathrm{~S}_{0}$ & $\times 10^{-4}$ & $1.9 \pm 0.2$ & 2.92 & & 1.90 & $2.25 \pm 0.44$ & 2.25 \\
$\left\langle\Gamma_{\gamma 0}\right\rangle$ & $\mathrm{meV}$ & $97 \pm 8$ & 98.0 & & 97.0 & & 100.0 \\
\hline$\left\langle\mathrm{D}_{1}\right\rangle$ & $\mathrm{eV}$ & & 3.53 & & 1.97 & & $2.24^{* *}$ \\
$\mathrm{~S}_{1}$ & $\times 10^{-4}$ & & 0.76 & & 1.10 & & 1.60 \\
$\left\langle\Gamma_{\gamma 1}\right\rangle$ & $\mathrm{meV}$ & & 98.0 & & 97.0 & & 100.0 \\
\hline$\left\langle\mathrm{D}_{2}\right\rangle$ & $\mathrm{eV}$ & & & & 1.26 & & \\
$\mathrm{~S}_{2}$ & $\times 10^{-4}$ & & & & 2.30 & & \\
$\left\langle\Gamma_{\gamma 2}\right\rangle$ & $\mathrm{meV}$ & & & & 97.0 & & \\
\hline
\end{tabular}

* Average parameters at the low energy of the unresolved region $(304 \mathrm{eV})$.

** At the neutron separation energy of ${ }^{158} \mathrm{Gd}$. 


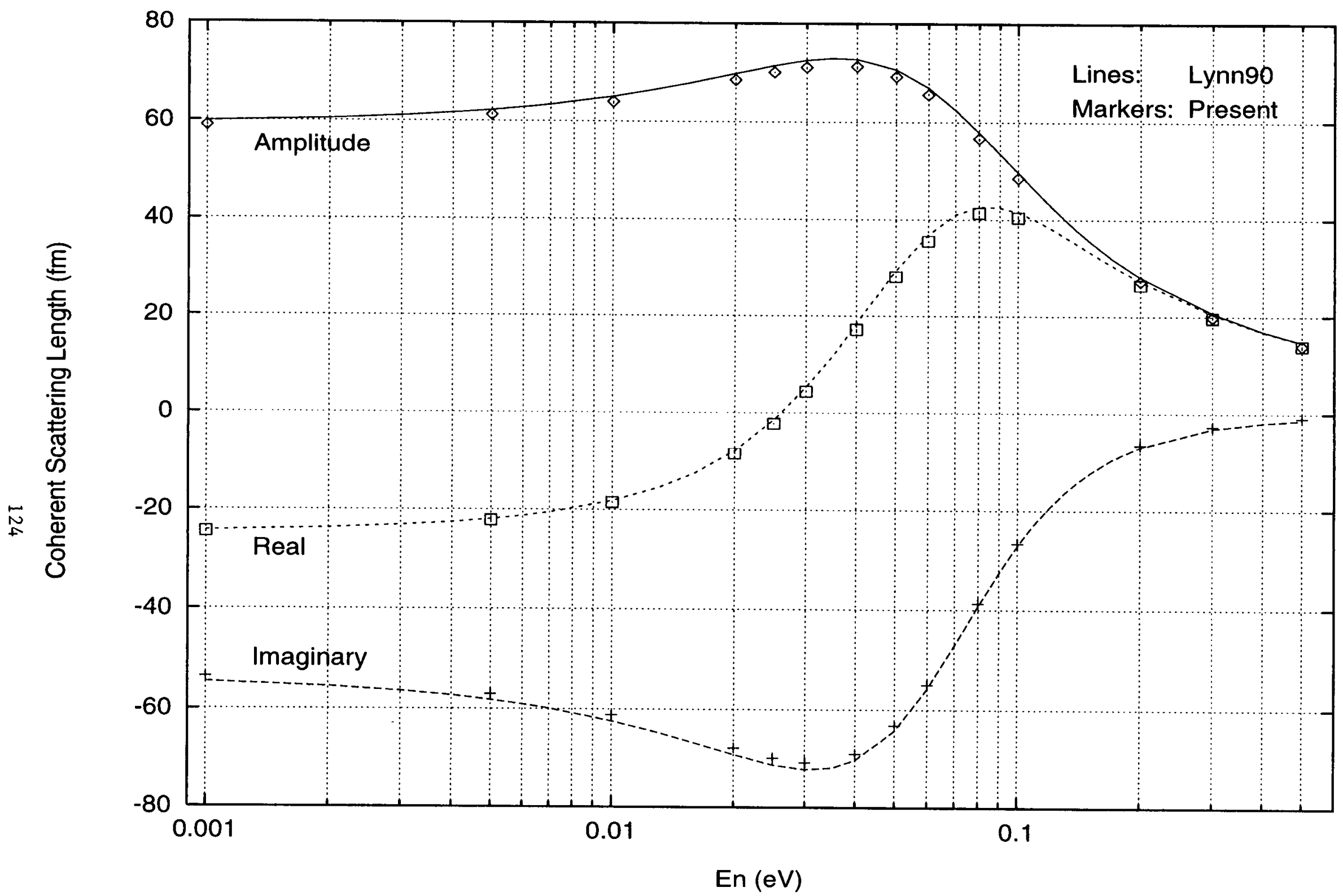

Fig. 56. Bound Coherent Scattering Lengths of ${ }^{157} \mathrm{Gd}$ 


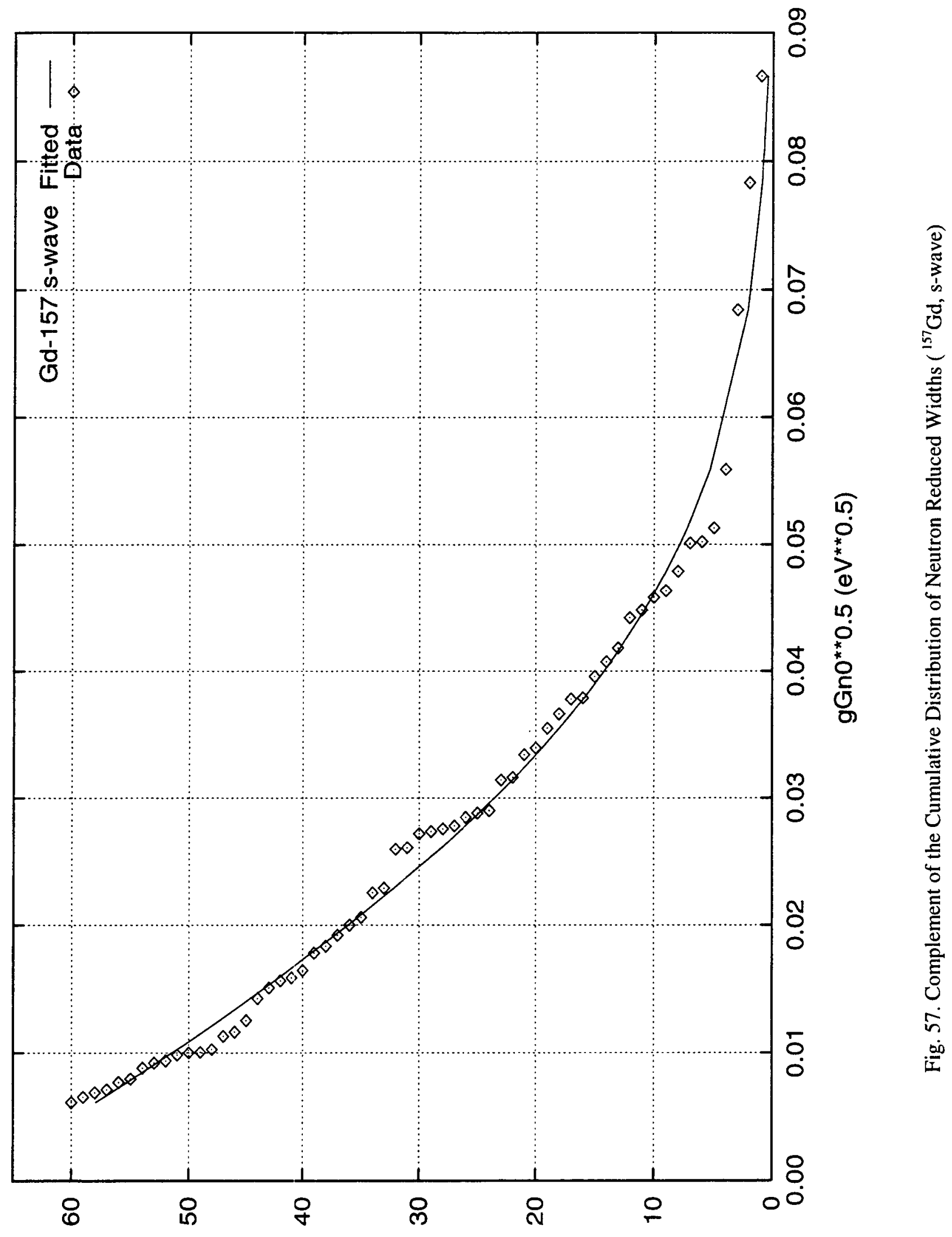

səoueuosəy to "ON əর!๋̣|nuñ 


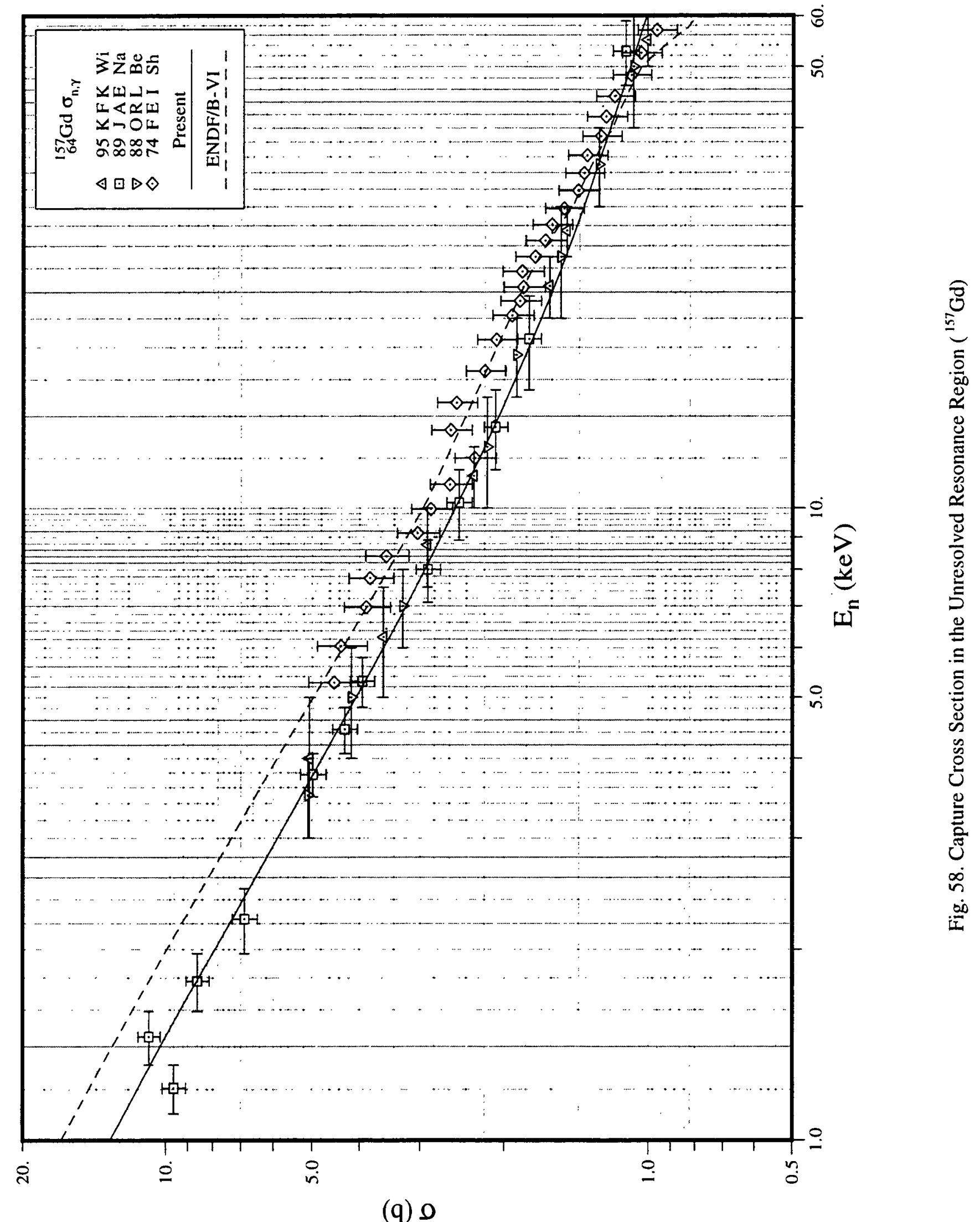




\section{COMPARISONS WITH OTHER EVALUATIONS}

In this section, we present a brief over-all summary of the evaluations of the 19 fissionproduct nuclei and compare our results with previous evaluations, high-lighting the major differences and disagreements with other evaluations.

\section{IV.A Resolved and Unresolved Resonance Data Files}

Table 46 gives a summary of the resolved and unresolved energy regions of the present and ENDF/B-VI evaluations. As shown for ${ }^{99} \mathrm{Tc},{ }^{103} \mathrm{Rh},{ }^{105} \mathrm{Pd},{ }^{109} \mathrm{Ag},{ }^{131} \mathrm{Xe},{ }^{133} \mathrm{Cs}{ }^{141} \mathrm{Pr},{ }^{147} \mathrm{Sm}$, and ${ }^{149} \mathrm{Sm}$, the resonance regions of the present evaluations are represented by more additional resonances than those of the ENDF/B-6 evaluations.

\section{IV.B Thermal Cross Sections}

In Table 47, the thermal capture cross sections of the nuclei ${ }^{95} \mathrm{Mo},{ }^{105} \mathrm{Pd},{ }^{145} \mathrm{Nd},{ }^{147} \mathrm{Sm}$, and

${ }^{150} \mathrm{Sm}$ display absolute differences greater than $3 \%$ between the present evaluations and those of ENDF/B-6. However, good agreement is achieved with other evaluations for these isotopes.

\section{IV.C Capture Resonance Integrals}

As demonstrated in Table 48, disagreements larger than $\pm 5 \%$ between the present results and those of ENDF/B-6 exist in the calculated capture resonance integrals for ${ }^{99} \mathrm{Tc},{ }^{105} \mathrm{Pd},{ }^{131} \mathrm{Xe},{ }^{131} \mathrm{Cs}$, and ${ }^{141} \mathrm{Pr}$.

\section{IV.D Thermal Elastic Cross Sections}

Table 49 shows that the calculated thermal scattering cross sections of the present study are larger than any of the evaluations for ${ }^{95} \mathrm{Mo},{ }^{105} \mathrm{Pd}$, and ${ }^{151} \mathrm{Sm}$ while for ${ }^{131} \mathrm{Xe}$ it is smaller than the other evaluations.

\section{IV.E Effective Scattering Radii}

The potential scattering radii implemented in the resolved and unresolved energy regions of the various evaluations are summarized in Table 50. With the exception of ${ }^{155} \mathrm{Gd}$ and ${ }^{157} \mathrm{Gd}$, general agreement exits among the various evaluations. However, our values for these two isotopes, which are consistent with the systematic study [Mu84], are much larger than those of the other evaluations.

\section{IV.F Wescott Factors for Capture Cross Sections}

As shown in Table 51, there is good agreement between the present calculations of the Wescott factors for capture and those of other evaluations. 


\section{IV.G Wescott Factors for Scattering and Total Cross Sections}

Table 52 gives a summary of the calculated scattering and total resonance integrals of the various evaluations. Major disagreements, larger than $15 \%$, exist among the various evaluations of the scattering resonance integrals for ${ }^{101} \mathrm{Ru},{ }^{105} \mathrm{Pd},{ }^{150} \mathrm{Sm},{ }^{151} \mathrm{Sm},{ }^{155} \mathrm{Gd}$, and ${ }^{157} \mathrm{Gd}$.

\section{IV.H S-Wave Strength Functions}

In Table 53, the s-wave neutron strength functions, implemented in the unresolved-energy regions of the various evaluations, as well as those reported in [Mu81, Mu84, Re98], are compared. Within the uncertainty limits generated by the Porter-Thomas analysis in the resolved energy region, general agreement exists among the different evaluations

\section{IV.I S-Wave Radiative Widths}

In Table 54, the s-wave average level spacings, implemented in the unresolved energy region of the various evaluations, as well as those of [Mu81], [Mu84] and [Re98] are compared. With the exception of ${ }^{95} \mathrm{Mo},{ }^{143} \mathrm{Nd}$ and ${ }^{152} \mathrm{Sm}$, the level spacings of the present study, derived from the resolved energy region, are applied in the unresolved energy region without any adjustments.

\section{IV.J S-Wave Radiative Widths}

In Table 55, the s-wave average radiative widths, implemented in the unresolved energy regions of the various evaluations, as well as those reported in [Mu81], [Mu84] and [Re98], are compared. In the present study, the average values obtained from the resolved energy region were generally applied in the unresolved region. Varying adjustments in the radiative widths were carried out in order to fit the unresolved capture cross section. Adjustments larger than $20 \%$ were made on the radiative widths of ${ }^{99} \mathrm{Tc},{ }^{145} \mathrm{Nd}$, and ${ }^{149} \mathrm{Sm}$, none on ${ }^{101} \mathrm{Ru},{ }^{105} \mathrm{Pd},{ }^{131} \mathrm{Xe},{ }^{133} \mathrm{Cs},{ }^{147} \mathrm{Sm},{ }^{150} \mathrm{Sm},{ }^{153} \mathrm{Eu}$, ${ }^{155} \mathrm{Gd}$, and ${ }^{157} \mathrm{Gd}$, and minor ones on the remainder.

\section{IV.K Marwellian Capture Cross Sections at $30 \mathrm{KeV}$}

Because of interest in astrophysical calculations, we computed the Maxwellian capture cross sections at a temperature of $30 \mathrm{keV}$ on the basis of the present evaluated capture cross sections, as well as those of ENDF/B-6. The results are included in Table 56 and are compared with Beer's [Be92] and Bao's [Ba87] compilations. When compared with the results derived on the basis of the ENDF/B-6 evaluations, differences larger than $10 \%$ exist for ${ }^{103} \mathrm{Rh},{ }^{143} \mathrm{Nd},{ }^{149} \mathrm{Sm}$. However, our results for these nuclei are in good agreement with those of [Be92] and [Ba87]. 
Table 46. Status of Resonance Data in $M F=2$

\begin{tabular}{|c|c|c|c|c|c|c|c|}
\hline \multirow{2}{*}{ Isotope } & \multirow{2}{*}{$\begin{array}{l}\text { Ist Inelastic } \\
\text { Excited Level } \\
(\mathrm{keV})^{a}\end{array}$} & \multicolumn{2}{|c|}{ Upper Energy of RRR (keV) } & \multicolumn{2}{|c|}{ No. of Resolved Resonances ${ }^{b}$} & \multicolumn{2}{|c|}{ Upper Energy of URR (keV) } \\
\hline & & ENDF/B-VI & Present & ENDF/B-VI & Present & ENDF/B-VI & Present \\
\hline 42-Mo-95 & 204.12 & 2.188 & 2.141 & $25+30(0)$ & $20+35(1)$ & - & 206.3 \\
\hline 43-Tc-99 & 140.51 & 0.800 & 0.983 & $42+25(1)$ & $41+54(1)$ & 141.4 & 141.9 \\
\hline $44-R u-101$ & 127.23 & 1.000 & 1.035 & $40+0(0)$ & $41+7(0)$ & 100.0 & 128.5 \\
\hline $45-\mathrm{Rh}-103$ & 39.756 & 1.500 & 4.116 & $59+60(0)$ & $101+178(1)$ & 40.4 & 40.2 \\
\hline 46-Pd-105 & 280.51 & 1.000 & 2.054 & $80+0(1)$ & $141+57(2)$ & - & 283.2 \\
\hline 47-Ag-109 & 88.034 & 2.509 & 4.996 & $83+0(0)$ & $236+71(0)$ & - & 88.8 \\
\hline $54-X \mathrm{e}-131$ & 80.185 & 4.000 & 3.945 & $39+0(1)$ & $45+2(1)$ & - & 80.8 \\
\hline $55-\mathrm{Cs}-133$ & 80.997 & 2.500 & 3.989 & $123+0(0)$ & $148+39(2)$ & - & 81.6 \\
\hline 59-Pr-141 & 145.44 & 0.991 & 10.049 & $15+0(0)$ & $79+47(2)$ & - & 146.5 \\
\hline $60-\mathrm{Nd}-143$ & 742.05 & 5.285 & 5.503 & $121+27(1)$ & $122+27(1)$ & 100.0 & 225.0 \\
\hline $60-\mathrm{Nd}-145$ & 67.220 & 4.140 & 3.979 & $212+0(1)$ & $194+0(1)$ & 50.0 & 67.7 \\
\hline $62-\mathrm{Sm}-147$ & 121.22 & 1.000 & 1.998 & $140+0(1)$ & $211+0(1)$ & 30.0 & 122.1 \\
\hline $62-\mathrm{Sm}-149$ & 22.507 & $0.100^{c}$ & 0.520 & $29+0(1)$ & $158+0(1)$ & 10.0 & $100.0^{d}$ \\
\hline $62-\mathrm{Sm}-150$ & 333.86 & 1.600 & 1.563 & $22+0(1)$ & $22+0(1)$ & 100.0 & 336.1 \\
\hline $62-\mathrm{Sm}-151$ & 4.821 & 0.300 & 0.296 & $120+0(1)$ & $120+0(1)$ & - & $66.2^{e}$ \\
\hline $62-\mathrm{Sm}-152$ & 121.78 & 5.025 & 5.102 & $91+0(1)$ & $91+0(1)$ & 100.0 & 122.6 \\
\hline $63-\mathrm{Eu}-153$ & 83.367 & 0.097 & 0.098 & $71+0(1)$ & $71+0(1)$ & 1.0 & 83.9 \\
\hline $64-G d-155$ & 60.009 & 0.183 & 0.183 & $92+0(0)$ & $92+0(0)$ & 10.4 & 60.4 \\
\hline $64-G d-157$ & 54.533 & 0.307 & 0.307 & $56+0(0)$ & $60+0(0)$ & 10.4 & 54.9 \\
\hline
\end{tabular}

a: In the center-of-mass system

b: Number of s-wave positive-energy resonances + Number of p-wave resonances; Number of bound level resonances in parentheses

c: Lower limit of the resolved resonance region(RRR) is $2.361 \mathrm{eV}$.

$\mathrm{d}$ : The second inelastic level is $277.075 \mathrm{keV}$ in the center-of-mass system.

e: This corresponds to the second inelastic level. 
Table 47. Capture Cross Sections at $0.0253 \mathrm{eV}$ (barn)

\begin{tabular}{|c|c|c|c|c|c|c|c|c|}
\hline Isotope & $\begin{array}{c}\text { BNL } \\
\text { Compilation }\end{array}$ & 98CRC & ENDF/B-VI ${ }^{a}$ & JEF-2.2 & JENDL-3. $2^{b}$ & LIPAR-5 & Present $^{c}$ & $\begin{array}{c}\text { Relative } \\
\text { Diff. (\%) }\end{array}$ \\
\hline 42-Mo-95 & $14.0 \pm 0.5$ & $13.4 \pm 0.3$ & 14.6 & 14.0 & 14.0 & - & 13.6 & -7.4 \\
\hline 43-Tc-99 & $20 \pm 1$ & $23 \pm 2$ & 19.6 & 19.1 & 19.6 & - & 20.0 & 2.0 \\
\hline $44-R u-101$ & $3.4 \pm 0.9$ & $5 \pm 1$ & 3.43 & 3.42 & 3.36 & - & 3.45 & 0.6 \\
\hline $45-\mathrm{Rh}-103$ & $145 \pm 2$ & 145 & 147 & 146 & 147 & - & 145 & -1.4 \\
\hline 46-Pd-105 & $20.0 \pm 3.0$ & $22 \pm 2$ & 20.1 & 21.8 & 20.3 & - & 20.9 & 3.8 \\
\hline 47-Ag-109 & $91.0 \pm 1.0$ & 91.2 & 91.0 & 90.8 & 90.5 & 90.7 & 90.8 & -0.2 \\
\hline $54-\mathrm{Xe}-131$ & $85 \pm 10$ & $90 \pm 10$ & 90.6 & 85.1 & 85.0 & - & 90.0 & -0.7 \\
\hline $55-\mathrm{Cs}-133$ & $29.0 \pm 1.5$ & 30.4 & 29.7 & 29.1 & 29.0 & - & 29.0 & -2.4 \\
\hline 59-Pr-141 & $11.5 \pm 0.3$ & 11.5 & 11.5 & 11.5 & 11.5 & - & 11.5 & 0.0 \\
\hline $60-\mathrm{Nd}-143$ & $325 \pm 10$ & $330 \pm 10$ & 325 & 323 & 330 & 319 & 325 & 0.0 \\
\hline $60-\mathrm{Nd}-145$ & $42 \pm 2$ & $47 \pm 6$ & 42.1 & 41.9 & 43.8 & 41.9 & 49.8 & 15.5 \\
\hline $62-S m-147$ & $57 \pm 3$ & $56 \pm 4$ & 57.5 & 57.2 & 58.0 & 56.7 & 50.0 & -15.0 \\
\hline $62-S m-149$ & $40140 \pm 600$ & $40100 \pm 600$ & 39730 & 40480 & 40150 & 39420 & 40530 & 2.0 \\
\hline $62-\mathrm{Sm}-150$ & $104 \pm 4$ & $102 \pm 5$ & 104 & 103 & 109 & 108.2 & 100 & -3.9 \\
\hline $62-\mathrm{Sm}-151$ & $15200 \pm 300$ & $15200 \pm 300$ & 15250 & 15190 & 15160 & 15160 & 15170 & -0.5 \\
\hline $62-\mathrm{Sm}-152$ & $206 \pm 6$ & $206 \pm 15$ & 207 & 206 & 206 & 202 & 206 & -0.5 \\
\hline 63-Eu-153 & $312 \pm 7$ & $300 \pm 20$ & 313 & 300 & 313 & 312 & 312 & 0.0 \\
\hline $64-G d-155$ & $60900 \pm 500$ & $61000 \pm 1000$ & 61100 & 60790 & 60890 & 60710 & 60730 & -0.6 \\
\hline $64-G d-157$ & $254000 \pm 815$ & $254000 \pm 3000$ & 255800 & 253400 & 254100 & 253500 & 253700 & -0.8 \\
\hline
\end{tabular}

a: From JEF Report 14, OECD/NEA, Paris (1994)

b: From General Description (MF=1) of JENDL-3.2

c: Calculated using LINEAR-RECENT-SIGMA1-INTER codes

d: Relative Difference $=\left(1-\sigma_{\gamma}{ }^{\mathrm{ENDF} / \mathrm{VI}} / \sigma_{\gamma}^{\text {Present }}\right) \times 100$ 
Table 48. Capture Resonance Integrals (barn)

\begin{tabular}{|c|c|c|c|c|c|c|c|c|}
\hline Isotope & $\begin{array}{c}\text { BNL } \\
\text { Compilation }\end{array}$ & 98CRC & ENDF/B-VI ${ }^{\mathrm{a}}$ & JEF-2.2 & JENDL-3. $2^{b}$ & LIPAR $^{\circ}{ }^{c}$ & Present ${ }^{d}$ & $\begin{array}{c}\text { Relative } \\
\text { Diff. (\%) }\end{array}$ \\
\hline 42-Mo-95 & $109 \pm 5$ & $109 \pm 5$ & 113 & 110 & 119 & - & 111 & -2.2 \\
\hline 43-Тc-99 & $340 \pm 20$ & - & 350 & 304 & 312 & - & 312 & -12.2 \\
\hline 44-Ru-101 & $100 \pm 20$ & $110 \pm 30$ & 111 & 111 & 100 & - & 111 & 0.1 \\
\hline 45-Rh-103 & $1100 \pm 50$ & 1180 & 1035 & 1035 & 1040 & - & 1036 & 0.1 \\
\hline 46-Pd-105 & 62.2 & $60 \pm 20$ & 111 & 93.1 & 96.8 & - & 95.2 & -16.6 \\
\hline 47-Ag-109 & $1400 \pm 48$ & 1480 & 1471 & 1473 & 1470 & 1467 & 1476 & 0.4 \\
\hline $54-X e-131$ & $900 \pm 100$ & $900 \pm 100$ & 1016 & 890 & 900 & - & 882 & -15.2 \\
\hline $55-C s-133$ & $437 \pm 26$ & 422 & 383 & 439 & 396 & - & 421 & 9.0 \\
\hline 59-Pr-141 & $17.4 \pm 2.0$ & $14 \pm 3$ & 19.0 & 17.9 & 18.4 & - & 17.6 & -7.7 \\
\hline $60-N d-143$ & $128 \pm 30$ & $128 \pm 30$ & 130 & 130 & 130 & 127 & 130 & 0.7 \\
\hline $60-N d-145$ & $240 \pm 35$ & $260 \pm 40$ & 231 & 231 & 204 & 228 & 245 & 5.7 \\
\hline $62-\mathrm{Sm}-147$ & - & $710 \pm 50$ & 789 & 794 & 781 & 721 & 777 & -1.4 \\
\hline $62-\mathrm{Sm}-149$ & 3390 & $3100 \pm 500$ & 3258 & 3484 & 3490 & 3355 & 3482 & 6.4 \\
\hline $62-\mathrm{Sm}-150$ & $358 \pm 50$ & $290 \pm 30$ & 338 & 339 & 325 & 334 & 334 & -1.2 \\
\hline $62-\mathrm{Sm}-151$ & $3520 \pm 160$ & $3520 \pm 60$ & 3449 & 3465 & 3410 & 3397 & 3430 & -0.6 \\
\hline $62-\mathrm{Sm}-152$ & $2970 \pm 100$ & $3000 \pm 300$ & 2981 & 2977 & 2770 & 2958 & 2976 & -0.2 \\
\hline 63-Eu-153 & $1420 \pm 100$ & $1800 \pm 400$ & 1499 & 1448 & 1410 & 1305 & 1408 & -6.5 \\
\hline $64-G d-155$ & $1447 \pm 100$ & $1540 \pm 100$ & 1555 & 1543 & 1540 & 1437 & 1537 & -1.2 \\
\hline $64-G d-157$ & $700 \pm 20$ & $800 \pm 100$ & 759 & 762 & 763 & 711 & 754 & -0.7 \\
\hline
\end{tabular}

a: From JEF Report 14, OECD/NEA, Paris (1994)

b: From General Description (MF=1) of JENDL-3.2

c: Integrated from $0.5 \mathrm{eV}$ to the upper energy of resolved resonance region

d: Calculated using LINEAR-RECENT-SIGMA1-INTER codes; Integrated from $0.5 \mathrm{eV}$ to $100 \mathrm{keV}$ with $1 / \mathrm{E}$ spectrum

e: Relative Difference $=\left(1-\mathrm{I}_{\gamma}^{\text {ENDF/B-VI }} / \mathrm{I}_{\gamma}^{\text {Present }}\right) \times 100$ 
Table 49. Elastic Scattering Cross Sections at $0.0253 \mathrm{eV}$ (barn)

\begin{tabular}{|c|c|c|c|c|c|c|}
\hline Isotope & $\begin{array}{c}\text { BNL } \\
\text { Compilation }\end{array}$ & ENDF/B-VI & $\mathrm{JEF}-2.2^{\mathrm{a}}$ & JENDL-3. $2^{b}$ & LIPAR-5 & Present $^{c}$ \\
\hline 42-Mo-95 & - & 2.48 & 5.21 & $5.57^{\circ}$ & - & 6.41 \\
\hline 43-Тc-99 & - & 6.88 & 3.54 & 3.44 & - & 5.81 \\
\hline $44-R u-101$ & - & 3.23 & 5.05 & 3.74 & - & 4.50 \\
\hline 45-Rh-103 & - & 4.69 & 3.43 & 3.28 & - & 4.37 \\
\hline $46-\mathrm{Pd}-105$ & $5.0 \pm 0.6$ & 5.01 & 5.22 & 5.13 & - & 7.36 \\
\hline 47-Ag-109 & $2.55 \pm 0.06$ & 2.25 & 2.30 & 2.48 & 2.42 & 2.12 \\
\hline $54-\mathrm{Xe}-131$ & - & 4.32 & 24.3 & 24.0 & - & 1.19 \\
\hline $55-\mathrm{Cs}-133$ & - & 4.98 & 3.88 & 4.29 & - & 3.97 \\
\hline 59-Pr-141 & $2.54 \pm 0.06$ & 2.18 & 2.59 & 2.54 & - & 2.71 \\
\hline $60-N d-143$ & $80 \pm 2$ & 80.6 & 80.8 & 80.3 & 80.0 & 80.4 \\
\hline $60-\mathrm{Nd}-145$ & - & 17.4 & 18.3 & 20.3 & 17.9 & 18.7 \\
\hline 62-Sm-147 & - & 39.1 & 39.9 & 1.06 & 3.8 & 1.06 \\
\hline 62-Sm-149 & 197 & 139 & 176 & 173 & 163 & 194 \\
\hline $62-\mathrm{Sm}-150$ & - & 19.0 & 21.2 & 8.34 & 10.3 & 27.7 \\
\hline $62-S m-151$ & - & 43.5 & 39.3 & 50.4 & 34.4 & 62.0 \\
\hline $62-\mathrm{Sm}-152$ & $3.0 \pm 0.2$ & 3.12 & 2.93 & 0.95 & 7.49 & 3.11 \\
\hline 63-Eu-153 & $9.7 \pm 0.7$ & 9.04 & 6.75 & 10.3 & 7.68 & 9.00 \\
\hline $64-G d-155$ & 60 & 58.9 & 59.0 & 59.0 & 58.6 & 60.7 \\
\hline $64-G d-157$ & 1000 & 1015 & 1756 & 1007 & 1005 & 1009 \\
\hline
\end{tabular}

a: From JEF Report 14, OECD/NEA, Paris (1994)

b: From General Description (MF=1) of JENDL-3.2

c: Calculated using LINEAR-RECENT-SIGMAI-INTER codes 
Table 50. Effective Scattering Radii (fm)

\begin{tabular}{|c|c|c|c|c|c|c|c|c|}
\hline \multirow{2}{*}{ Isotope } & \multirow{2}{*}{$\begin{array}{c}\text { BNL } \\
\text { Compilation }\end{array}$} & \multicolumn{2}{|c|}{ ENDF/B-VI } & \multicolumn{2}{|c|}{ JEF-2.2 } & \multicolumn{2}{|c|}{ JENDL-3.2 } & \multirow{2}{*}{ Present } \\
\hline & & In RRR & In URR & In RRR & In URR & In RRR & In URR & \\
\hline 42-Mo-95 & $7.0 \pm 0.2$ & 5.50 & & 7.00 & & 7.00 & 6.68 & 7.00 \\
\hline 43-Тc-99 & $6.0 \pm 0.5$ & 7.91 & $\leftarrow$ & 6.00 & 6.80 & 6.00 & 6.22 & 7.00 \\
\hline 44-Ru-101 & & 5.62 & $\leftarrow$ & 6.90 & $\leftarrow$ & 6.10 & 5.06 & 6.53 \\
\hline 45-Rh-103 & $6.2 \pm 0.3$ & 7.09 & $\leftarrow$ & 6.20 & 6.56 & 6.20 & 6.52 & 6.56 \\
\hline 46-Pd-105 & $6.6 \pm 0.3$ & 6.60 & & 6.70 & $\leftarrow$ & 6.50 & 4.60 & 6.60 \\
\hline 47-Ag-109 & $6.6 \pm 0.2$ & 6.60 & & 6.60 & 6.30 & 7.05 & 6.62 & 6.60 \\
\hline $54-X e-131$ & & 5.85 & & 5.40 & & 5.31 & 5.63 & 5.40 \\
\hline $55-\mathrm{Cs}-133$ & $5.3 \pm 0.5$ & 7.52 & & 5.30 & 5.50 & 5.35 & 5.84 & 5.30 \\
\hline 59-Pr-141 & $4.9 \pm 0.5$ & 6.28 & & 4.40 & $\leftarrow$ & 4.90 & 5.18 & 4.93 \\
\hline $60-\mathrm{Nd}-143$ & & 5.54 & $\leftarrow$ & 5.60 & 7.34 & 5.60 & 4.13 & 5.60 \\
\hline $60-\mathrm{Nd}-145$ & & 6.80 & $\leftarrow$ & 6.00 & 5.00 & 6.00 & 7.68 & 6.00 \\
\hline $62-\mathrm{Sm}-147$ & $8.3 \pm 0.2$ & 8.30 & $\leftarrow$ & 8.30 & 6.37 & 8.30 & 6.64 & 8.30 \\
\hline 62-Sm-149 & $8.3 \pm 0.2$ & 5.09 & $\leftarrow$ & 8.30 & 5.09 & 7.52 & 7.90 & 8.30 \\
\hline $62-\mathrm{Sm}-150$ & & 6.41 & $\leftarrow$ & 6.40 & & 8.08 & 5.92 & 8.00 \\
\hline $62-\mathrm{Sm}-151$ & & 8.30 & & 7.20 & 7.46 & 7.95 & 5.72 & 8.30 \\
\hline $62-\mathrm{Sm}-152$ & $8.3 \pm 0.2$ & 8.30 & 7.67 & 8.30 & 6.44 & 8.20 & 7.56 & 8.30 \\
\hline 63-Eu-153 & $8.2 \pm 0.2$ & 8.80 & $\leftarrow$ & 8.80 & $\leftarrow$ & 8.63 & 6.42 & 8.20 \\
\hline 64-Gd-155 & & 6.70 & $\leftarrow$ & 6.70 & 3.59 & 6.70 & 6.83 & 8.00 \\
\hline 64-Gd-157 & & 4.90 & $\leftarrow$ & 4.90 & & 4.90 & 6.88 & 7.80 \\
\hline
\end{tabular}

* The same value is adopted for both resolved and unresolved resonance regions. 
Table 51. Westcott Factors for Capture

\begin{tabular}{|c|c|c|c|c|c|}
\hline Isotope & ENDF/B-VI & JEF-2.2 & JENDL-3.2 ${ }^{\star}$ & LIPAR-5 & Present $^{b}$ \\
\hline 42-Mo-95 & 0.9990 & 0.9987 & 0.9994 & - & 1.0000 \\
\hline 43-Tc-99 & 1.004 & 1.004 & 1.004 & - & 1.0036 \\
\hline 44-Ru-101 & 1.003 & 1.001 & 1.001 & - & 1.0016 \\
\hline $45-\mathrm{Rh}-103$ & 1.022 & 1.023 & 1.022 & - & 1.0229 \\
\hline 46-Pd-105 & 0.9973 & 0.9951 & 0.9986 & - & 0.9995 \\
\hline 47-Ag-109 & 1.006 & 1.005 & 1.005 & 1.0050 & 1.0053 \\
\hline $54-\mathrm{Xe}-131$ & 0.9996 & 1.001 & 1.001 & - & 1.0014 \\
\hline $55-\mathrm{Cs}-133$ & 1.004 & 1.002 & 1.002 & - & 1.0029 \\
\hline 59-Pr-141 & 1.002 & 0.9997 & 0.9990 & - & 0.9995 \\
\hline $60-\mathrm{Nd}-143$ & 0.9981 & 0.9961 & 0.9961 & 0.9961 & 0.9966 \\
\hline $60-\mathrm{Nd}-145$ & 1.002 & 0.9996 & 1.000 & 0.9998 & 1.0000 \\
\hline $62-\mathrm{Sm}-147$ & 0.9990 & 1.001 & 0.9946 & 0.9942 & 0.9965 \\
\hline $62-\mathrm{Sm}-149$ & 1.704 & 1.717 & 1.685 & 1.7088 & 1.7171 \\
\hline $62-S m-150$ & 1.000 & 0.9979 & 0.9938 & 0.9936 & 0.9985 \\
\hline 62-Sm-151 & 0.9308 & 0.9271 & 0.9278 & 0.9256 & 0.9274 \\
\hline $62-\mathrm{Sm}-152$ & 1.004 & 1.003 & 1.003 & 1.0032 & 1.0035 \\
\hline 63-Eu-153 & 0.9738 & 0.9662 & 0.9869 & 0.9801 & 0.9871 \\
\hline 64-Gd-155 & 0.8419 & 0.8435 & 0.8437 & 0.8446 & 0.8440 \\
\hline 64-Gd-157 & 0.8500 & 0.8514 & 0.8522 & 0.8527 & 0.8515 \\
\hline
\end{tabular}

a: Calculated with values of $\sigma_{y}{ }^{0}$ and $\sigma_{\gamma, \text { Maxw }}$ in JEF Report 14, OECD/NEA, Paris (1994)

b: Calculated using LINEAR-RECENT-SIGMA1-INTER codes 
Table 52. Elastic Scattering and Total Resonance Integrals*

\begin{tabular}{|c|c|c|c|c|c|c|c|c|}
\hline \multirow{2}{*}{ Isotope } & \multicolumn{4}{|c|}{ Elastic Scattering Resonance Integral (barn) } & \multicolumn{4}{|c|}{ Total Resonance Integral (barn) } \\
\hline & ENDF/B-VI & JEF-2.2 & JENDL-3.2 & Present & ENDF/B-VI & JEF-2.2 & JENDL-3.2 & Present \\
\hline 42-Mo-95 & 178 & 196 & 216 & 205 & 291 & 306 & 334 & 315 \\
\hline 43-Tc-99 & 113 & 84.7 & 74.8 & 97.1 & 463 & 389 & 386 & 409 \\
\hline 44-Ru-101 & 72.9 & 89.6 & 73.9 & 89.3 & 184 & 200 & 173 & 200 \\
\hline 45-Rh-103 & 94.1 & 79.8 & 78.5 & 89.5 & 1129 & 1115 & 1121 & 1126 \\
\hline 46-Pd-105 & 88.7 & 82.2 & 72.1 & 90.8 & 200 & 175 & 168 & 186 \\
\hline 47-Ag-109 & 219 & 216 & 218 & 214 & 1691 & 1690 & 1689 & 1691 \\
\hline $54-\mathrm{Xe}-131$ & 1862 & 2068 & 2039 & 1883 & 2877 & 2958 & 2938 & 2765 \\
\hline $55-C s-133$ & 135 & 117 & 113 & 114 & 518 & 556 & 508 & 535 \\
\hline $59-\operatorname{Pr}-141$ & 284 & 216 & 221 & 220 & 303 & 234 & 239 & 237 \\
\hline $60-\mathrm{Nd}-143$ & 597 & 597 & 567 & 598 & 726 & 726 & 696 & 728 \\
\hline $60-\mathrm{Nd}-145$ & 567 & 566 & 573 & 562 & 797 & 798 & 776 & 807 \\
\hline $62-S m-147$ & 871 & 845 & 745 & 728 & 1660 & 1639 & 1524 & 1505 \\
\hline 62-Sm-149 & 560 & 485 & 578 & 538 & 3819 & 3969 & 4071 & 4021 \\
\hline $62-S m-150$ & 492 & 501 & 475 & 556 & 830 & 840 & 799 & 890 \\
\hline $62-\mathrm{Sm}-151$ & 267 & 297 & 343 & 325 & 3726 & 3765 & 3752 & 3765 \\
\hline $62-\mathrm{Sm}-152$ & 6074 & 6070 & 5977 & 6068 & 9055 & 9048 & 8740 & 9045 \\
\hline 63-Eu-153 & 199 & 196 & 196 & 181 & 1699 & 1644 & 1605 & 1590 \\
\hline 64-Gd-155 & 171 & 162 & 134 & 168 & 1726 & 1705 & 1667 & 1705 \\
\hline $64-G d-157$ & 200 & 182 & 175 & 234 & 960 & 946 & 936 & 989 \\
\hline
\end{tabular}

* Integrated from $0.5 \mathrm{eV}$ to $100 \mathrm{keV}$ with 1/E spectrum: Present values are calculated using LINEAR-RECENT-SIGMA l-INTER codes.

Others are from JEF Report 14, OECD/NEA, Paris (1994). 
Table 53. s-wave Neutron Strength Functions in the Unresolved Resonance Region $\left(\times 10^{4}\right)$

\begin{tabular}{|c|c|c|c|c|c|c|c|}
\hline \multirow{2}{*}{ Isotope } & \multirow{2}{*}{$\begin{array}{c}\text { BNL } \\
\text { Compilation }\end{array}$} & \multirow{2}{*}{ ENDF/B-VI } & \multirow{2}{*}{ JEF-2.2 } & \multirow{2}{*}{ JENDL-3.2* } & \multirow{2}{*}{ RIPL } & \multicolumn{2}{|c|}{ Present } \\
\hline & & & & & & PT Analysis & Adopted \\
\hline $42-\mathrm{Mo}-95$ & $0.35 \pm 0.07$ & - & - & 0.37 & $0.60 \pm 0.10$ & $0.45 \pm 0.16$ & 0.45 \\
\hline 43-Тc-99 & $0.45 \pm 0.05$ & 0.43 & 0.55 & 0.54 & $0.48 \pm 0.07$ & $0.43 \pm 0.10$ & 0.43 \\
\hline 44-Ru-101 & $0.54 \pm 0.04$ & 0.59 & 0.59 & 0.59 & $0.56 \pm 0.05$ & $0.62 \pm 0.15$ & 0.62 \\
\hline $45-\mathrm{Rh}-103$ & $0.6 \pm 0.1$ & 0.43 & 0.49 & 0.45 & $0.47 \pm 0.06$ & $0.57 \pm 0.09$ & 0.57 \\
\hline 46-Pd-105 & $0.60 \pm 0.05$ & - & 0.58 & 0.60 & $0.60 \pm 0.05$ & $0.66 \pm 0.08$ & 0.50 \\
\hline 47-Ag-109 & $0.46 \pm 0.15$ & - & 0.64 & 0.54 & $0.75 \pm 0.08$ & $0.52 \pm 0.05$ & 0.57 \\
\hline $54-\mathrm{Xe}-131$ & $1.2 \pm 0.4$ & - & - & 0.70 & $1.20 \pm 0.30$ & $0.79 \pm 0.20$ & 0.79 \\
\hline $55-\mathrm{Cs}-133$ & $0.7 \pm 0.07$ & - & 0.80 & 0.70 & $0.76 \pm 0.10$ & $0.73 \pm 0.09$ & 0.73 \\
\hline $59-\operatorname{Pr}-141$ & $1.5 \pm 0.2$ & - & 1.00 & 1.50 & $1.70 \pm 0.30$ & $1.77 \pm 0.30$ & 1.77 \\
\hline $60-\mathrm{Nd}-143$ & $3.2 \pm 0.3$ & 3.50 & 3.40 & 2.62 & $3.80 \pm 0.40$ & $3.62 \pm 0.51$ & 3.62 \\
\hline $60-\mathrm{Nd}-145$ & $4.4 \pm 0.4$ & 4.00 & 5.20 & 2.93 & $3.20 \pm 0.40$ & $4.75 \pm 0.52$ & 4.75 \\
\hline $62-\mathrm{Sm}-147$ & $4.8 \pm 0.5$ & 4.80 & 4.26 & 4.80 & $4.80 \pm 0.50$ & $4.86 \pm 0.50$ & 4.86 \\
\hline $62-\mathrm{Sm}-149$ & $4.6 \pm 0.6$ & 3.20 & 4.13 & 4.60 & $6.30 \pm 1.20$ & $4.53 \pm 0.54$ & 4.53 \\
\hline $62-S m-150$ & $3.6 \pm 1.1$ & 3.60 & - & 3.60 & $3.40 \pm 0.60$ & $4.31 \pm 1.42$ & 4.31 \\
\hline $62-\mathrm{Sm}-151$ & $4.2 \pm 0.4$ & - & 4.50 & 4.20 & $3.40 \pm 0.50$ & $4.06 \pm 0.56$ & 4.06 \\
\hline $62-\mathrm{Sm}-152$ & $2.2 \pm 0.3$ & 2.50 & 2.30 & 2.20 & $3.00 \pm 0.40$ & $2.23 \pm 0.35$ & 2.23 \\
\hline $63-E u-153$ & $2.5 \pm 0.2$ & 2.50 & 2.50 & 2.80 & $2.20 \pm 0.30$ & $2.37 \pm 0.43$ & 2.37 \\
\hline $64-G d-155$ & $2.0 \pm 0.2$ & 3.08 & 3.00 & 2.00 & $2.00 \pm 0.30$ & $2.13 \pm 0.35$ & 2.13 \\
\hline $64-G d-157$ & $1.9 \pm 0.2$ & 2.92 & - & 1.90 & $2.20 \pm 0.40$ & $2.25 \pm 0.44$ & 2.25 \\
\hline
\end{tabular}

* At the lowest energy of the unresolved resonance region 
Table 54. s-wave Average Level Spacings in the Unresolved Resonance Region (eV)

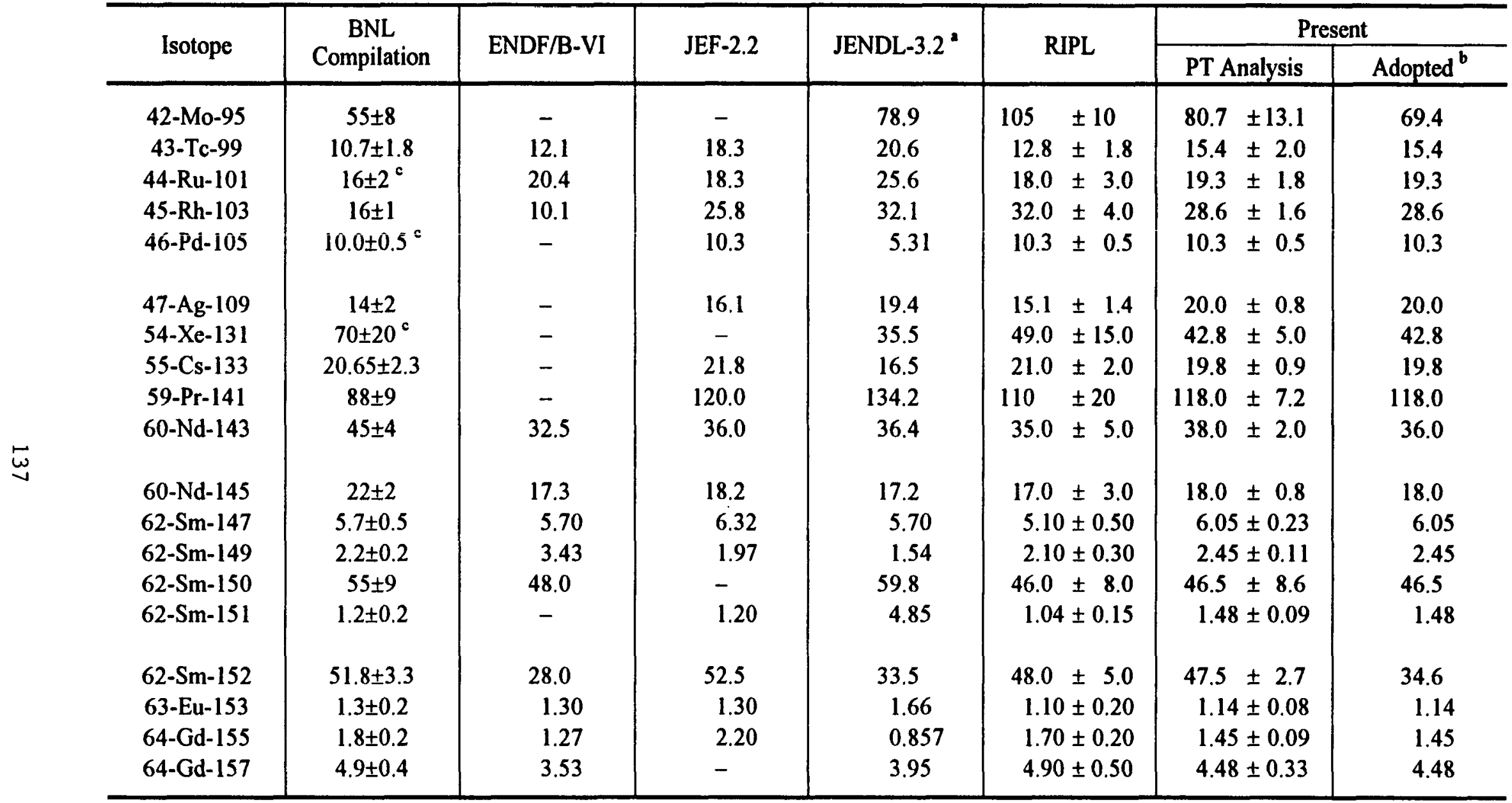

a: At the lowest energy of unresolved resonance region

b: At the neutron separation energy of target+n

c: Observed level spacing 
Table 55. s-wave Average Radiative Widths in the Unresolved Resonance Region (meV)

\begin{tabular}{|c|c|c|c|c|c|c|c|}
\hline \multirow{2}{*}{ Isotope } & \multirow{2}{*}{$\begin{array}{c}\text { BNL } \\
\text { Compilation }\end{array}$} & \multirow{2}{*}{ ENDF/B-VI } & \multirow{2}{*}{ JEF-2.2 } & \multirow{2}{*}{ JENDL-3.2 } & \multirow{2}{*}{ RIPL } & \multicolumn{2}{|c|}{ Present } \\
\hline & & & & & & Average " & Adopted \\
\hline 42-Mo-95 & $160 \pm 20$ & - & - & 232 & $150 \pm 20$ & $158 \pm 14$ & 150 \\
\hline 43-Tc-99 & 160 & 122 & 131.4 & 187 & $160 \pm 50$ & $173 \pm 16$ & 130 \\
\hline 44-Ru-101 & $180 \pm 15$ & 180 & 180 & 173 & $180 \pm 15$ & $184 \pm 10$ & 184 \\
\hline $45-\mathrm{Rh}-103$ & $160 \pm 15$ & 153 & 161.3 & 230 & $160 \pm 15$ & $162 \pm 28$ & 170 \\
\hline 46-Pd-105 & $145 \pm 8$ & - & 155 & 145 & $150 \pm 8$ & $148 \pm 4$ & 148 \\
\hline 47-Ag-109 & 130 & - & 132.3 & 130 & $130 \pm 20$ & $130 \pm 3$ & 133 \\
\hline $54-X e-131$ & - & - & - & 114 & $114 \pm 37$ & $112 \pm 11$ & 112 \\
\hline $55-C s-133$ & 120 & - & 125.3 & 120 & $120 \pm 10$ & $123 \pm 4$ & 123 \\
\hline 59-Pr-141 & - & - & 85.0 & 86 & $88 \pm 9$ & $86.0 \pm 2.0$ & 86.0 \\
\hline $60-\mathrm{Nd}-143$ & $80 \pm 9$ & 80 & $68.0,78.0$ & 79.1 & $86 \pm 9$ & $79.9 \pm 2.0$ & 79.9 \\
\hline $60-\mathrm{Nd}-145$ & $54 \pm 5$ & 75 & 89.1 & 97.5 & $87 \pm 9$ & $63.8 \pm 3.8$ & $76.5^{b}$ \\
\hline $62-\mathrm{Sm}-147$ & $69 \pm 2$ & 69 & 87.6 & 69 & $69 \pm 2$ & $73.8 \pm 1.5$ & 73.8 \\
\hline $62-S m-149$ & $62 \pm 2$ & 62 & 75.1 & 62 & $62 \pm 2$ & $56.7 \pm 8.9$ & $78.5^{b}$ \\
\hline $62-\mathrm{Sm}-150$ & $60 \pm 5$ & 60 & - & 60 & $87 \pm 16$ & $60.0 \pm 0.02$ & 60.0 \\
\hline $62-\mathrm{Sm}-151$ & $92 \pm 7$ & - & 96 & 92 & $95 \pm 4$ & $92.9 \pm 3.7$ & 92.9 \\
\hline $62-\mathrm{Sm}-152$ & $61 \pm 7$ & 61 & 71.8 & 61 & $67 \pm 5$ & $60.0 \pm 5.4$ & 72.7 \\
\hline $63-\mathrm{Eu}-153$ & $93 \pm 3$ & 95.8 & 95.8 & 94 & $95 \pm 12$ & $93 \pm 2$ & 93.0 \\
\hline $64-G d-155$ & $110 \pm 3$ & 112 & 120 & 110 & $108 \pm 10$ & $114 \pm 3$ & 114 \\
\hline $64-G d-157$ & $97 \pm 8$ & 98 & - & 97 & $97 \pm 22$ & $100 \pm 8$ & 100 \\
\hline
\end{tabular}

a: Uncertainty-weighted average of known radiative widths of resolved resonances

b: Unweighted average radiative width 
Table 56. Average Capture Cross Sections Weighted with Maxwellian Spectrum at $30 \mathrm{keV}(\mathrm{mb})$

\begin{tabular}{|c|c|c|c|c|c|c|}
\hline \multirow{2}{*}{ Isotope } & \multicolumn{3}{|c|}{ Measured } & \multicolumn{3}{|c|}{ Calculated ${ }^{8}$} \\
\hline & Beer's Compilation ${ }^{b}$ & Bao's Compilation ${ }^{c}$ & Recent Data & ENDF/B-VI & Present & Relative Diff. (\%) \\
\hline Mo-95 & $292 \pm 12$ & $374 \pm 50$ & & 375 & 377 & 0.4 \\
\hline Tc-99 & $782 \pm 39$ & $779 \pm 40$ & & 855 & 795 & -7.5 \\
\hline $\mathrm{Ru}-10 \mathrm{l}$ & $996 \pm 40$ & $996 \pm 40$ & & 952 & 970 & 1.8 \\
\hline Rh-103 & $810 \pm 14$ & $875 \pm 35$ & & 943 & 836 & -12.7 \\
\hline Pd-105 & $1199 \pm 60$ & $1199 \pm 60$ & & 1150 & 1190 & 3.3 \\
\hline Ag-109 & $779 \pm 23$ & $779 \pm 23$ & & 807 & 787 & -2.6 \\
\hline $\mathrm{Xe}-131$ & $453 \pm 81$ & 348 & & 291 & 306 & 5.1 \\
\hline Cs-133 & $509 \pm 21$ & $509 \pm 21$ & & 552 & 513 & -7.5 \\
\hline Pr-141 & $119 \pm 15$ & $119 \pm 15$ & & 115 & 117 & 1.7 \\
\hline Nd-143 & $242 \pm 10$ & $253 \pm 10^{d}$ & $244.6 \pm 3.1^{e}$ & 287 & 239 & -19.9 \\
\hline $\mathrm{Nd}-145$ & $485 \pm 100$ & $485 \pm 100$ & $424.8 \pm 4.5^{\circ}$ & 444 & 423 & -5.0 \\
\hline $\mathrm{Sm}-147$ & $1005 \pm 100$ & $1005 \pm 100$ & $973.1 \pm 10.0^{f}$ & 931 & 929 & -0.2 \\
\hline Sm-149 & $1409 \pm 65$ & $1454 \pm 66$ & $1819.9 \pm 17.2^{f}$ & 2384 & 1866 & -27.8 \\
\hline Sm-150 & $434 \pm 26$ & $447 \pm 26$ & $421.9 \pm 3.8^{f}$ & 378 & 409 & 7.5 \\
\hline $\mathrm{Sm}-151$ & $1932 \pm 206$ & 1932 & $1825 \pm 450^{\mathrm{g}}$ & 2466 & 2569 & 4.0 \\
\hline Sm-152 & $401 \pm 24$ & $396 \pm 22^{d}$ & $473.2 \pm \quad 4.4^{\mathrm{f}}$ & 494 & 460 & -7.4 \\
\hline Eu-153 & $3170 \pm 317$ & $3170 \pm 317$ & & 2575 & 2486 & -3.5 \\
\hline Gd-155 & $2721 \pm 90$ & $2800 \pm 280$ & $2648 \pm 30^{h}$ & 2592 & 2564 & -1.1 \\
\hline Gd-157 & $1355 \pm 39$ & $1538 \pm 154$ & $1369 \pm 15^{h}$ & 1387 & 1361 & -2.0 \\
\hline
\end{tabular}

a: Using INTER; integrated from $10^{-5} \mathrm{eV}$ to $1 \mathrm{MeV}$. b: Beer, H., Voss, F. and Winters, R.R., Astrophys. J. Suppl. Ser. 80, 403 (1992) c: Bao, Z.Y. and Kaeppeler, F., Atomic Data and Nucl. Data Tables 36, 411 (1987) d: Renormalized value in Ratynski, W. and Kaeppeler F., Phys. Rev. C37, 595 (1988) e: Wisshak, K. et al., Phys. Rev. C57, 391 (1998) f: Wisshak, K. et al., Phys. Rev. C48, 1401 (1993)

g: Calculated value in Toukan, K.A. et al., Phys. Rev. C51, 1540 (1995) h: Wisshak, K. et al., Phys. Rev. C52, 2762 (1995) 
This page has been intentionally left blank. 


\section{REFERENCES}

[Ab97] Abagyan, L.P., "The LIPAR Resonance Parameter Library," INDC(CCP)-406, IAEA (1997).

[Ai61] Aitken, K.L. and Cornish, F.W., "The Neutron Capture Cross Sections of Sm-149 and Sm-150," J. Inorg. Nucl. Chem. 17, 6 (1961).

[Ak76] Akopian, G.G. et al., "Neutron Spin States Amplitudes and Scattering Cross-Sections for Rare-Earth Nuclei," Proc. Int'l Conf. on Interactions of Neutrons with Nuclei, Vol. 2, p.1243, Lowell, July 6-9 (1976).

[A182] Allen, B.J. et al., "Stable Isotope Capture Cross Sections from the Oak Ridge Electron Linear Accelerator - Part II," Nucl. Sci. Eng., 82, 230 (1982).

[A182b] Alfimenkov, V.P. et al., "Investigation of Spin Dependence of Neutron Cross Sections and of Strenghth Functions for Rare Earth Nuclei in Experiments with Polarized Neutrons and Nuclei," Nucl. Phys. A376, 229 (1982).

[An78] Anufriev, V.A. et al., "Resonance Parameters of ${ }^{133} \mathrm{Cs}$ and ${ }^{134} \mathrm{Cs}$ Isotopes at Energies up to $400 \mathrm{eV}$," Sov. Atomic Enegy 43, 828 (1978).

[An79] Anufriev, V.A. et al, Atom. Energiya 47, 269 (1979).

[An80] Antonov, A.V. et al., Proc. $5^{\text {th }}$ All-Union Conf. on Nucl. Phys., p.125, Sept. 15-19, Kiev (1980).

[An85] Anufriev, V.A. et al., "Total Neutron Cross Section of ${ }^{101} \mathrm{Ru}$ and ${ }^{102} \mathrm{Ru}$ in the Neutron Energy Range 0.01- $1750 \mathrm{eV}$," Sov. Atomic Energy 58, 326 (1985).

[Ba87] Bao, Z.Y. and Kaeppeler, F., "Neutron Capture Cross Sections for s-Process Studies," Atomic Data and Nucl. Data Tables 36, 411 (1987).

[Be88] Beer, H. and Macklin, R.L., "The ${ }^{151} \mathrm{Sm}$ Branching; A Probe for the Irradiation Time Scale of the s-Process," Astrophys. J. 331, 1047 (1988).

[Be90] Belyaev, F.N. et al., "Study of ${ }^{155,157}$ Gd Resonances by $\gamma$ ray Multiplicity," Sov. J. Nucl. Phys. 52, 401 (1990).

[Be92] Beer, H. et al., "On the Calculation of Maxwellian-Averaged Capture Cross Sections," Astrophys. J. Suppl. Ser., 80, 403 (1992).

[Bo68] Bollinger, L.M. and Thomas, G.E., "p-Wave Resonances of $U^{238}$," Phys. Rev. 171, 1293 (1968).

[Bo85] Bokhovko, M.V. et al., Yad. Konst. 1985(3), 12 (1985).

[Bo85b] Bokhovko, M.V. et al., "Neutron Radiation Cross-Section, Neutron Transmission and Average Resonance Parameters for Some Fission Product Nuclei," Yad. Konst. 1985(2), 44 (1985).

[Bo87] Bokhovko, M.V. et al., "Measurement of the Neutron Capture Cross-Sections for Silver in the Energy Region $4-400 \mathrm{keV}$," INDC(CCP)-280/L, p.15 (Translation of Yad. Konst., 1987(2), 21 (1987)).

[Bo91] Bokhovko, M.V. et al., "Average Fast Neutron Radiative Capture Cross-Sections for Fission Products and for Isotopes of Rare Earth Elements," Proc. Int 'l Conf. Nucl. Data for Sci. Technol., p.62, Juelich, May 13-17 (1991).

[Bu82] Buyl, R. and Corvi, F., "Thermal Capture Measurements of ${ }^{105} \mathrm{Pd}$ and ${ }^{108} \mathrm{Pd}$," NEANDC(E) 232U, Vol. III, p. 18 (1982).

[Ca71] Cauvin, B. et al, Proc. of 3rd Conf. Neutron Cross Sections and Technology, p.785, March 15-17, Knoxville (1971). 
[Ca71b] Carlson, A.D. and Fricke, M.P., UC-34, Gulf Radiation Tech. (1971).

[Ch73] Chou, J.C. and Werle, H., J. Nucl. Energy 27, 811 (1973).

[Co82] Cornelis, E. et al., "Average Capture Cross Section of the Fission Product Nuclei 104,105,106,108 Pd," Proc. Int. Conf. on Nucl. Data for Sci. and Technol., p.222, Antwerp, Sept. 6-10 (1982).

[Co97] Corvi, F., private communication. Also see: Corvi, F., et al., "Parity Dependence of Low Level Populations in the ${ }^{107} \mathrm{Ag}(\mathrm{n}, \gamma)$ and ${ }^{109} \mathrm{Ag}(\mathrm{n}, \gamma)$ Resonance Reactions," Proc. Int'l Conf. Nucl. Data for Sci. Technol., p.731, Trieste, May 19-24 (1997).

[Da67] Dakowski, M. et al., Nucl. Phys. A97, 187 (1967).

[De95] M.D. DeHart, 1995. "Sensitivity and Parametric Evaluations of Significant Aspects of Burnup Credit for PWR Spent Nuclear Fuel Packages," ORNL/TM-12973, Oak Ridge National Laboratory, Martin Marietta Energy Systems, Inc. (to be published).

[Dy63] Dyson, F.J. and Mehta, M.L., "Statistical Theory of the Energy Levels of Complex Systems. IV," J. Mathematical Phys., 4, 701 (1963).

[Ei74] Eiland, H.M. et al., "Neutron Cross Sections of Samarium-147 and Samarium-150," Nucl. Sci. Eng. 54, 286 (1974).

[EX] http://www.nea.fr/html/dbdata/x4 or http://www.nndc.bnl.gov/nndc/exfor

[Fe67] Fenner, N.C. and Large, R.S., "Measurement of the Neutron Capture Cross Sections of ${ }^{147} \mathrm{Pm},{ }^{148} \mathrm{Pm},{ }^{148 m} \mathrm{Pm}$ and ${ }^{147} \mathrm{Sm}$, J. Inorg. Nucl. Chem. 29, 2147 (1967).

[Fi78] Fischer, P. et al., Neutron Physics and Nuclear Data, p.718, Harwell (Sept. 1978).

[Fr70] Fiesenhan, S.J. et al., Nucl. Phys. A146, 337.

[Ga81] Garg, J.B., Tikku, V.K., and Harvey, J.A., Phys. Rev. C23, 671 (1981).

[Ge92] Georgiev, G.P. et al., JNR-P3-92-346, Joint Institute of Nuclear Research (1992).

[Ge93] Georgiev, G. at al., "Determination of ${ }^{147} \mathrm{Sm}$ and ${ }^{148} \mathrm{Sm}$ Resonance Parameters," Nucl. Phys. A565, 643 (1993).

[Gi61] Gibbons, J.H. et al., "Average Radiative Capture Cross Sections for 7- to 170-keV Neutrons," Phys. Rev., 122, 182 (1961).

[G179] Glaettli, H. et al., "Experimental Values of Spin Dependent Nuclear Scattering Lengths of Slow Neutrons," J. de Physique 40, 629 (1979).

[G187] Glaettli, H. et al., "Spin-Dependent Scattering Lengths Slow Neutron-Nucleous by Pseudo-Magnetic Measurements," Z. Phys. A- Atomic Nuclei, 327, 149 (1987).

[Gu97] Gunsing, F. et al., Proc. Int. Conf. On Nucl. Data for Sci. Technol., p.1293, 19-24 May 1997, Trieste. (1997).

[Ha62] Halperin, J. et al., "Thermal Neutron Cross Section and Resonance Integral of Sm150," Trans. Amer. Nucl. Soc. 5, 376 (1962).

[Ha65] Halperin, J. et al., "Neutron Capture Cross Sections of ${ }^{99,100,101}{ }^{R u}$," ORNL-3832, p.4 (1965).

[Ha75] Haste, T.J. and Thomas, B.W., J. Phys. G: Nucl. Phys. 1, 981 (1975).

[Ha95] Harada, H. et al., J. Nucl. Sci. Tech. 32, 395 (1995).

[Ho75] Hockenbury, R.W. et al., COO-2479-4, Rensselaer Polytechnic Institute (1975).

[Ho75b] Hockenbury, R.W. et al., "Fission Product Capture Cross Sections in the keV Region," Proc. Conf. Nucl. Cross Section and Technol., March 3-7, Washington, p.905 (1975).

[Ho98] Holden, N.E., "Neutron Scattering and Absorption Properties," CRC Handbook of Chemistry and Physics, $78^{\text {th }}$ Ed., CRC Press (1998). 
[Ka64] Kapchigashev, S.P. and Popov, Yu.P., "Neutron Capture Cross Section Measurements for Several Molybdenum Isotopes," Proc. Conf. on Neutron Interactions, p.104, Dubna (1964).

[Ka69] Karzhavina, E.N. et al., Sov. J. of Nucl. Phys. 8, 371 (1969).

[Ko64] Konks, V.A. et al., "Cross Sections for Radiative Capture of Neutrons with Energies up to $50 \mathrm{keV}$ by La-139, Pr-141, Ta-181, and Au-197," Soviet Phys., 19, 56 (1964).

[Ko68] Konks, V.A. et al., "Radiative Capture of Neutrons by Nuclei with A=140-200," Sov. J. Nucl. Phys., 7, 310 (1968) (EXFOR 40362).

[Ko77] Kononov, V.N. et al., "Fast-Neutron Capture Cross Sections for Indium, Tantalum, Gold, Samarium, and Europium," Sov. J. Nucl. Phys., 26, 500 (1977).

[Ko78] Kononov, V.N. et al., "Fast-Neutron Capture Cross Sections for Even-Even Isotopes of Neodymium, Samarium, Gadolinium, and Erbium," Sov. J. Nucl. Phys. 27, 5 (1978).

[Ko81] Kononov, V.N. et al., "Fast-Neutron Radiative Capture Cross Sections and d-wave Strength Functions," Proc. $4^{\text {th }}$ Int'l Sympo. Neutron Capture Gamma-Ray Spectroscopy and Related Topics, p.518, Grenoble, Sept. 7-11 (1981).

[Ko87a] Koester, L. et al., "Interaction of Slow Neutrons with the Isotopes of Molybdenum," Z. Phys. A-Atomic Nuclei, 326, 227 (1987).

[Ko87b] Koester, L. et al., "Experimental Study on the 3p Size Resonance in the p-wave Neutron Strength Function," Z. Phys. A- Atomic Nuclei, 326, 185 (1987).

[Ko91] Koester, L. et al., "Neutron Scattering Lengths: A Survey of Experimental Data and Method," Atom. Data and Nucl. Data Tables, 49, 65 (1991).

[Li77] Little, R.C. and Block, R.C., Trans. Amer. Nucl. Soc. 26, 574 (1977).

[Lo97] Lowie, L.Y. et al., "Neutron Resonance Spectroscopy of ${ }^{107} \mathrm{Ag}$ and ${ }^{109} \mathrm{Ag}$," Phys.Rev. C, 56, 90 (1997).

[Lu77] Lucas, M. et al., Proc. Mtg. of the Technical Committee on Natural Fission Reactors, Vol. 1, p.431, Dec.19-21, Paris (1977).

[Lu94] Luo Xiao-bing et al., "Measurement of Neutron Capture Cross Section for ${ }^{152} \mathrm{Sm}$," Chinese J. Nucl. Phys., 16, 275 (1994).

[Ly90] Lynn, J.E. and Seeger, P.A., "Resonance Effects in Neutron Scattering Lengths of Rare-Earth Nuclides," At. Data Nucl. Data Tables 44, 191 (1990).

[Ma63] Macklin, R.L. et al., "Neutron Capture in the Samarium Isotopes and the Formation of the Element of the Solar System," Nature 197, 369 (1963).

[Ma79] Macklin, R.L. et al., “104,105,106,108,109 Pd(n, $\gamma$ ) Cross Sections Above $2.6 \mathrm{keV}$," Nucl. Sci. Eng., 71, 182 (1979).

[Ma80] Macklin, R.L. and Halperin, J., “100,101,102,104 $\mathrm{Ru}(\mathrm{n}, \gamma)$ and ${ }^{103} \mathrm{Rh}(\mathrm{n}, \gamma)$ Cross Sections above $2.6 \mathrm{keV}$," Nucl. Sci. Eng. 73, 174 (1980).

[Ma81] Macklin, R.L. and Winters, R.R., "Stable Isotope Capture Cross Sections from the Oak Ridge Electron Linear Accelerator," Nucl. Sci. Eng. 78, 110 (1981).

[Ma82] Macklin, R.L., Nucl. Sci. Eng. 81, 520 (1982).

[Ma82b] Macklin, R.L., "Neutron Capture Cross Sections of the Silver Isotopes ${ }^{107} \mathrm{Ag}$ and ${ }^{109} \mathrm{Ag}$ from 2.6 to $2000 \mathrm{keV}$," Nucl. Sci. Eng., 82, 400 (1982).

[Ma82c] Macklin, R.L., "Cesium-133 Neutron Capture Cross Section," Nucl. Sci. Eng. 81, 418 (1982).

[Ma86] Macklin, R.L., EXFOR 12966 (1986).

[Ma87] Macklin, R.L. and Young, P.G., "Neutron Capture Cross Sections of ${ }^{151} \mathrm{Eu}$ and ${ }^{153} \mathrm{Eu}$ from 3 to $2200 \mathrm{keV}$," Nucl. Sci. Eng., 95, 189 (1987) (EXFOR 12873). 
[Mc88] McLane, V. et al., Neutron Cross Sections, Vol. 2: Neutron Cross Section Curves, Academic Press (1988).

[Mi79a] Mizumoto, M. et al., "Low-Energy Neutron Resonances in ${ }^{206} \mathrm{~Pb}$," Phys. Rev. C19, 335 (1979).

[Mi79] Mizumoto, M. et al., "Average Neutron Capture Cross Sections of ${ }^{151}$ Eu and ${ }^{153}$ Eu from 3 to $100 \mathrm{keV}$," J. Nucl. Sci. Technol., 16, 711 (1979).

[Mi81] Mizumoto, M., "Neutron Resonance Parameters and Radiative Capture Cross Sections of ${ }^{147} \mathrm{Sm}$ and ${ }^{149} \mathrm{Sm}$," Nucl. Phys. A357, 90 (1981).

[Mi83] Mizumoto, M. et al., "Neutron Resonance Parameters of Silver-107 and Silver-109," J. Nucl. Sci. Technol., 20, 883 (1983).

[Mo60] Moeller, H.J. et al., Nucl. Sci. Eng. 8, 183 (1960).

[Mo69] Morgenstern, J. et al., "Parametres des Resonances et Fonctions Densites $S_{0}$ et $S_{1}$ pour $\mathrm{Cl},{ }^{51} \mathrm{~V},{ }^{89} \mathrm{Y}, \mathrm{Zr}, \mathrm{La},{ }^{141} \mathrm{Pr}$ et ${ }^{209} \mathrm{Bi}$," Nucl. Phys. A123, 561 (1969).

[Mo76] Moxon, M.C. et al., "The Neutron Capture Cross-Section of Eu-151 and Eu-153 in the Energy Range 0.1 to $100 \mathrm{keV,"} \mathrm{Ann.} \mathrm{Nucl.} \mathrm{Energy,} \mathrm{3,} 399$ (1976).

[Mu71] Mughabghab, S.F. et al., "Reaction Mechanism for p-Wave Neutron Capture in Mo" and $\mathrm{Mo}^{98}$," Phys. Rev. Letters, 26, 1118 (1971).

[Mu76] Musgrove, A.R. de L. et al., "Average Neutron Resonance Parameters and Radiative Capture Cross Sections for the Isotopes of Molybdenum," Nucl. Phys. A270, 108 (1976).

[Mu77] Musgrove, A.R. et al., AAEC/E401 (1977).

[Mu81] Mughabghab, S.F. et al., Neutron Cross Sections, Vol.1, Part A, Academic Press (1981).

[Mu84] Mughabghab, S.F., Neutron Cross Sections, Vol.1, Part B, Academic Press (1984).

[Mu98a] Mughabghab, S.F. and Dunford, C.L., Proc. Int. Conf. on Phys. of Nucl. Sci. and Technol., p.784, Long Island, Oct. 5-8 (1998).

[Mu98b] Mughabghab, S.F. and Dunford, C.L., Phys. Rev. Lett. 81, 4083 (1998).

[Mu99] Mughabghab, S.F. and Dunford, C.L., "A Unified Model for E1 Photon Strength Functions and the Landau Migdal Constants," BNL-NCS-66524 (1999). Also see "On the Questions of the Nuclear Level Density and the E1 Photon Strength Functions," Int. School on Nucl. Phys., Neutron Phys., and Nucl. Energy, Sept. 27 - Oct. 3, 1999, Varna, Bulgaria.

[Na78] Nakajima, Y. et al., Proc. Int. Conf. on Neutron Phys. and Nucl. Data for Reactors and Other Applied Purposes, p.438, Sept. 25-29, Harwell (1978).

[Na89] Nakajima, Y. et al., "Neutron Capture Cross Section Measurements of ${ }^{155} \mathrm{Gd}$ and ${ }^{157} \mathrm{Gd}$ from 1.1 to $235 \mathrm{keV}$," Ann. Nucl. Energy 16, 589 (1989).

[Na90] Nakajima, Y. et al., "Neutron Resonances in ${ }^{133}$ Cs," Ann. Nucl. Energy 17, 569 (1990).

[Oh99] Oh, S.Y., "The Probability Density Function of Neutron Reduced Width," Nuclear Data Evaluation Lab. Calculation Note, NDL-32/99, KAERI (Nov. 1999).

[Or70] Orphan, V.J. et al., "Line and Continuum Gamma-Ray Yields from Thermal-Neutron Capture in 75 Elements," GA-10248, Gulf General Atomic (1970).

[Pa58] Pattenden, N.J., Proc. Second UN Int. Conf. Peaceful Uses of Atomic Energy, v.16, p.44, Sept. 1-13, Geneva (1958).

[Pa92] Pandita, A. and Agrawal, H.M., "Statistical Analysis of s-Wave Neutron Reduced Widths," J. Phys. Soc. Japan, 61, 3524 (1992). 
[Po56] Porter, C.E. and Thomas, R.G., "Fluctuations of Nuclear Reaction Widths," Phys. Rev. 104, 483 (1956).

[Po79] Popov, A.B. et al., "Parameters of Ru neutron resonances," Sov. J. Nucl. Phys. 29, 285 (1979).

[Po81] Popov, A.B. and Tzeciak, K., "Parameters of Iodine and Cesium Neutron Resonances in 20-250 eV range," P3-81-721, JINR (1981).

[Pr86] Press, W.H. et al., Numerical Recipes, Section 14.4, Cambridge Univ. Press, Cambridge (1986).

[Ra97] Raepsaet, C. et al., Proc. Int. Conf. On Nucl. Data for Sci. Technol., p.1289, 19-24 May 1997, Trieste. (1997).

[Re98] Reffo, G., "Average Neutron Resonance Parameters," in Reference Input Parameter Library, IAEA-TECDOC-1034, p.25 (1998).

[Ri69] Ribon, P., "Etudes des Quelques Proprietes des Niveaus Excites des Noyaux Composes Formes par 103-Rh, Xe, 155-Gd, 157-Gd, 232-Th," CEA-N-1149 (1969) -Doctoral Thesis.

[Ri70] Ribon, P. et al., Nucl. Phys. A143, 130 (1970).

[Ro71] Rohr, G. et al., Proc. of 3rd Conf. Neutron Cross Sections and Technology, p.743, March 15-17, Knoxville (1971).

[Sh74] Shorin, V.S. et al., "Neutron Radiative-Capture Cross Sections in the Energy Region 5$70 \mathrm{keV}$ for Gd and Er Isotopes," Sov. J. Nucl. Phys. 19, 2 (1974).

[Sk96] Skoy, V.R. et al., "Isotopic Identification of the Parity-Violating Neutron p-wave Resonance at Energy $E_{0}=3.2 \mathrm{eV}$ in Xe," Phys. Rev. C53, 2573 (1996).

[Sm91] Smith, D.L., Probability, Statistics, and Data Uncertainties in Nuclear Science and Technology, American Nuclear Society (1991).

[St72] Stolovy, A. et al., Phys. Rev. C 5, 2030 (1972).

[St79] Staveloz, P. et al., "Neutron Resonance Parameters for Palladium Isotopes," Proc. Specialists' Mtg. on Neutron Cross Sections of Fission Product Nuclei," p.53, Bologna, Dec. 12-14 (1979); NEANDC(E) 209 L.

[Ta60] Tattersall, R.B. et al., J. Nucl. Energy/A 12, 32 (1960).

[Ta79] Taylor, R.B. et al., "keV Neutron Capture in ${ }^{141}$ Pr," Aust. J. Phys., 32, 551 (1979).

[Te71] Tellier, H., CEA-N-1459 (1971).

[To95] Toukan, K.A. et al., "Stellar Neutron Capture Cross Sections of Nd, Pm, and Sm Isotopes," Phys. Rev. C51, 1540 (1995).

[Ve74] Vertebnyi, V.P. et al., YFI-17, p.37, Fiziko-Energ. Institute, Obninsk (1974).

[Wa70] Watanabe, T. and Reeder, S.D., Nucl. Sci. Eng. 41, 188 (1970).

[Wi57] Wigner, E.P., Conf. on Neutron Physics by Time of Flight, Gatlinburg, 1956, p. 59, ORNL-2309 (1957).

[Wi86] Winters, R.R. et al., “ ${ }^{148,150} \mathrm{Sm}$ : A Test for s-process Nucleosynthesis," Astrophys. J. 300, 41 (1986).

[Wi87] Winters, R.R. and Macklin, R.L., "Maxwellian-Averaged Neutron Capture Cross Sections for ${ }^{99} \mathrm{Tc}$ and ${ }^{95-98} \mathrm{Mo}$," Astrophys. J., 313, 808 (1987).

[Wi90] Wisshak, K. et al., Phys. Rev. C42, 1731 (1990).

[Wi93] Wisshak, K. et al., "Neutron Capture in ${ }^{148,150} \mathrm{Sm}$ : A Sensitive Probe of the s-process Neutron Density," Phys.Rev. C48, 1401 (1993).

[Wi95] Wisshak, K. et al., "Stellar Neutron Capture Cross Sections of the Gd Isotopes," Phys. Rev. C52, 2762 (1995). 
[Wi98] Wisshak, K. et al., Phys. Rev. C 57, 391 (1998).

[Wo82] Word, R.E. and Werner, S.A., Phys. Rev. B 26, 4190 (1982).

[Wy68] Wynchank, S. et al., "Neutron Resonance Spectroscopy. VI. Mo, Sb, Te, and Pr," Phys. Rev., 166, 1234 (1968).

[Xi94] Xia Yijin et al., "Activation Cross Section Measurement for the Eu(n, $\gamma)$ Reactions," Proc. Int. Conf. Nucl. Data for Sci. Technol., May 9-13, Gatlinburg, Tennessee, p.251 (1994).

[Ya79] Yamamuro, N. and Asami, A. Proc. of Specialist's Mtg. in Neutron Cross Sections of Fission Product Nuclei, p.19, Dec. 12-14, Bologna, Italy (1979).

[Ya83] Yamamuro, N. et al., "KeV-Neutron Capture in Cesium-133, Gold-197 and Tantalum181," J. Nucl. Sci. Technol. 20, 797 (1983).

[Yu93] Yu Weixiang et al., "Activation Cross Section Measurement for the Eu(n,y) Reactions," Chinese J. Nucl. Phys., 15, 71 (1993).

[Za71] Zaikin, G.G. et al., "Fast-Neutron Radiative Capture Cross-Sections for the Isotopes ${ }^{69} \mathrm{Ga},{ }^{71} \mathrm{Ga},{ }^{139} \mathrm{La}$ and ${ }^{141} \mathrm{Pr}$," INDC(CCP)-15/U, p.52 (1971).

[Zh92] Zhao, Z. and Huang, Z., "Bayesian Approach to Evaluate Average Level Spacing," Proc. Int. Sympo. on Nucl. Data Eval. Methodology, p.327, Upton, Oct. 12-16 (1992). 


\section{APPENDICES}

\section{Appendix A: Evaluation Procedure}

Figure A1 describes the steps of the evaluation procedure and Fig. A2 presents a flow chart of the computer codes, which were used in accomplishing the various evaluation tasks. As a first step in the evaluation of each nuclide, the bibliographic information is retrieved from CINDA, the Computerized Index to Nuclear Data [CI], and Nuclear Science References [NS]. In addition, recent measured resonance parameters, as well as other data such as thermal cross sections and capture cross sections in the keV energy region, are obtained from CSISRS [CS], which is the NNDC version of EXFOR database [EX]. After reviewing the available measurements and updating the data reported in the BNL compilations, a computerized data file of resolved resonance parameters, $B N L 325 . T X T$, is prepared.

In the next step, as described in the main text, the $l$ and $\mathrm{J}$ values for resonances, which have not been determined from the measurements, are assigned by applying the Bayesian method and a random assignment method, respectively. In addition, the measured radiative widths are averaged and this averaged value is assigned to resonances with unknown radiative widths. The parameters for each resolved resonance are compiled into a File 2 in the ENDF- 6 format. The resulting file is named ENDFA.TXT. In addition, the fitting of reduced neutron widths to the Porter-Thomas distribution is performed. The computer code PTANAL (Fig. A2) performs all the tasks in this step. The resulting average parameters are then adopted in preparing the input file for WRIURR.

In the next step, a check is made as to whether the present resolved resonances reproduce reference thermal cross sections, scattering lengths, and resonance integrals. The thermal cross sections and scattering lengths are calculated by the PSY 325 computer code. If necessary, one or two bound levels are invoked. The bound levels are included in the file ENDFR.TXT. The thermal characteristics are checked again with PSY325, and, if necessary, the bound level parameters are adjusted to achieve agreement with the measurements. 
The energy-dependent average resonance parameters are then appended to the ENDFR.TXT by the WRIURR computer code. A set of parameters consists of energy dependent level spacing, reduced neutron width, and gamma width. The competition and fission widths are set as zero. Multiple sets of parameters, which show the energy dependence, are provided for each $l$ and J. The energy points are defined in the input file for WRIURR code. The file ENDFU.TXT is generated by WRIURR.

ENDFU.TXT is converted to the point-wise cross sections by the RECENT computer code. After extracting capture cross sections in the unresolved region from the endfu.pw file with the aid of PWC code, the calculated capture cross sections, together with measured data retrieved from EXFOR database, are plotted by the BNL325 plotting code. If the calculated cross sections do not show acceptable agreement with measured data, the resonance parameters for the unresolved region are then adjusted and a revised ENDFU.TXT file is then generated again. Note that the effect of Doppler broadening at a temperature of $300 \mathrm{~K}$ is negligible in the $\mathrm{keV}$ energy region.

To compare the present evaluation with other evaluated libraries or data sources, the thermal cross sections, resonance integrals, Maxwellian-averaged cross sections, and so on are calculated with the finalized ENDFU.TXT file. For this calculation, the point-wise cross sections are broadened to $300 \mathrm{~K}$ by using SIGMAil code. Subsequentiy, the INTER code caiculates the above quantities using the broadened.pw file resulting from SIGMA1.

Descriptions of the computer codes PSY325, LINEAR, RECENT, SIGMA1, and INTER can be found in [Le96].

\section{$\underline{\text { References }}$}

[CI] http://www.nndc.bnl.gov/nndc/cinda or http://www-nds.iaea.or.at/cinda

[CS] http://www.nndc.bnl.gov/nndc/exfor

[EX] http://www-nds.iaea.or.at/exfor

[Le96] Lemmel, H.D., "Index of Nuclear Data Libraries," IAEA-NDS-7, Rev. 96/11, IAEA (1996).

[NS] http://www.nndc.bnl.gov/nndc/nsr 


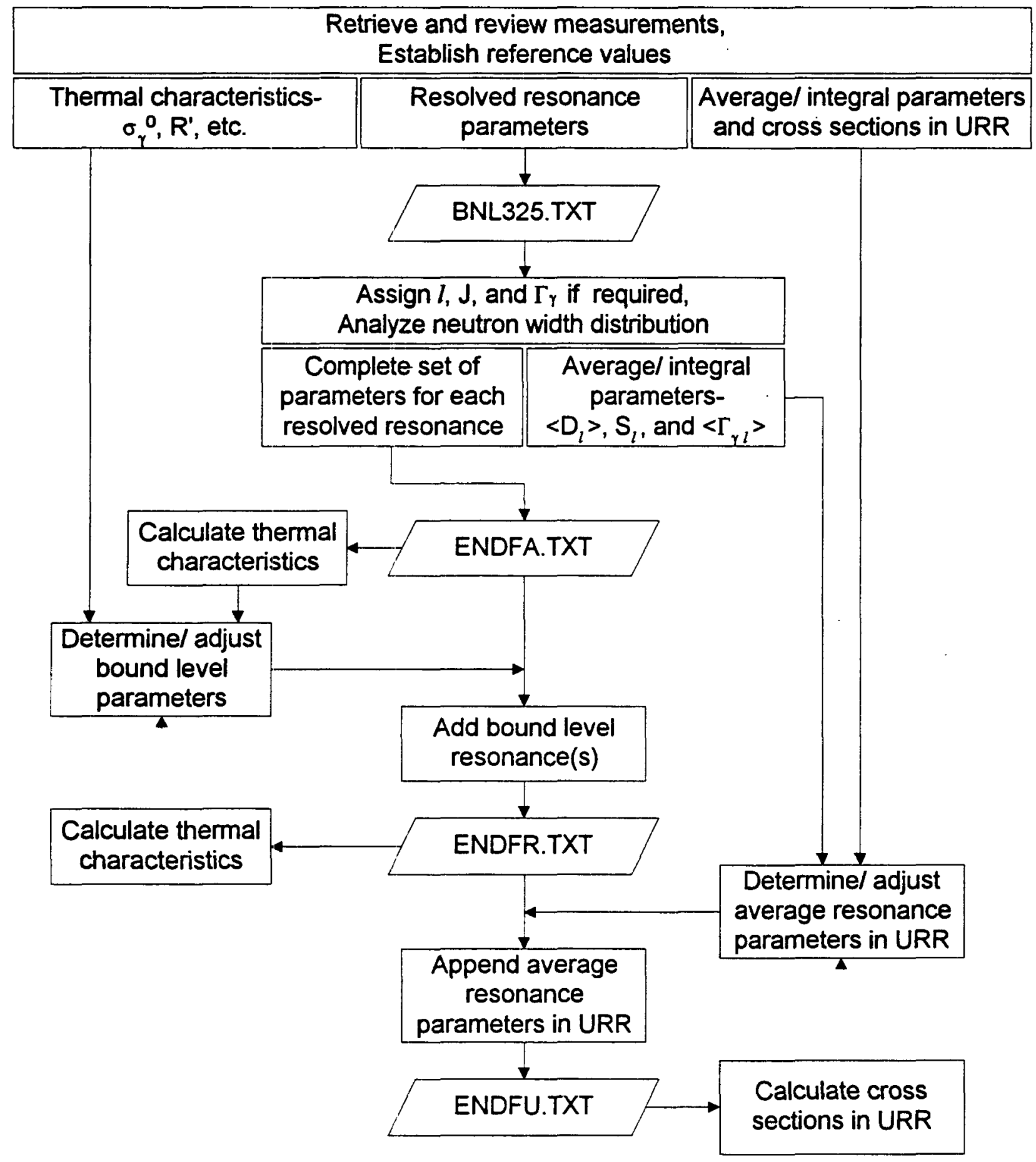

Figure Al. Evaluation Procedure 


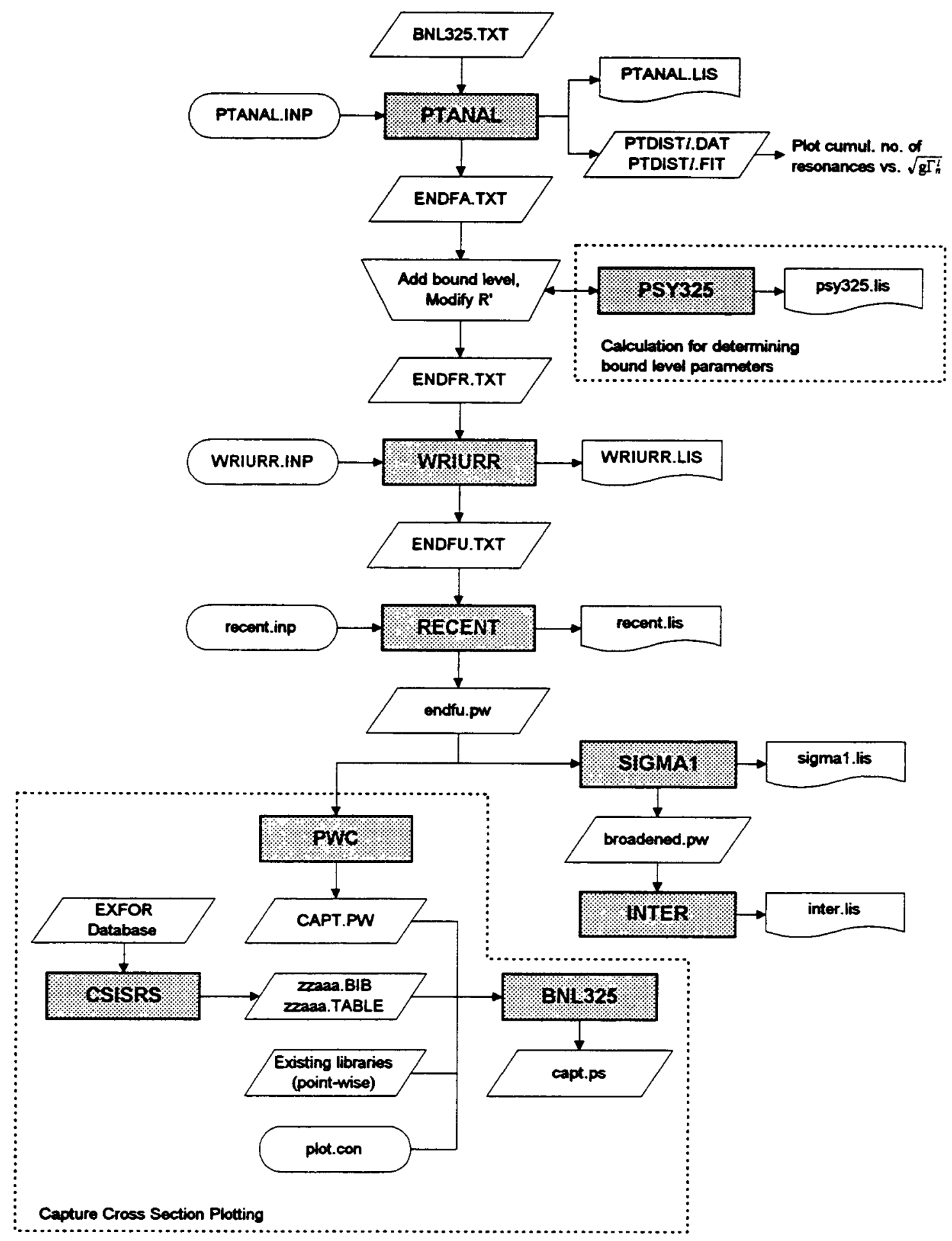

Figure A2. Flow of Computer Codes 


\section{Appendix B: PTANAL User's Guide}

The PTANAL program is used for obtaining the average resonance parameters from the analysis of the Porter-Thomas distribution for the reduced neutron widths in the resolved resonance energy region. It also assigns the $l$ and $\mathrm{J}$ values, which have not been determined from the measurements, by applying the Bayesian method and random assignment method, respectively. In addition, the known radiative widths are averaged in two ways: one is an arithmetic average and the other is the weighted-average with the inverse variance of the experimental value. Another purpose of PTANAL is to convert the resonance parameters from the CFMTA format into an ENDF-6 format for file 2.

The program requires two input files PTANAL.INP and BNL325.TXT. The PTANAL.INP file is the standard input in the name-list input format. This file consists of :

First card (in other words, record or line): "\&data" from column 2 to 6;

In the second to (last-1) cards: data section with the name-list keywords; and Last card: "\&end" from column 2 to 5.

The data section includes variables shown in Table B1. In the name list input, a user does not need to keep the order of variables. A sample input is shown at the end of Table B1.

The BNL325.TXT file contains recommended resonance parameters in CFMTA format. Two records are provided for each resonance as shown in Table B2. Flags indicating the status of given parameter are ' $R$ ' for recommended, ' $F$ ' for preferred, ' $A$ ' for assumed, ' '(blank) for undetermined. The PTANAL program deals only with the flags for the resonance spin and angular momentum. Unless the flag was set as ' $A$ ' or ' ', the program does not alter the $l$ value in the BNL325.TXT file regardless of the results of the Bayesian analysis. In the same context, the random assignment of $\mathrm{J}$ is applied only to resonances with the flag ' $A$ ' or "'.

The program generates output files PTANAL.LIS, ENDFA.TXT, PTDISTx.DAT, and PTDISTx.FIT files. The PTANAL.LIS is the standard output summarizing the results and ENDFA.TXT is the File 1 and 2 of the ENDF- 6 format containing resolved resonance parameters. The PTDISTx.DAT ( $x=$ null for all resonances, 0 for $s$-wave, or 1 for $p$-wave) files include the number of cumulative "measured" resonances vs. the neutron reduced widths multiplied by the spin statistical factor, $\mathrm{g}$, in the unit of $\mathrm{meV}$ in ascending order. The PTDISTx.FIT is same to .DAT file except "fitted" number of resonances. 
Table B1. Variables appearing in the PTANAL.INP file

\begin{tabular}{|c|c|c|c|}
\hline Name & Unit & Description & $\begin{array}{l}\text { Default } \\
\text { Value }\end{array}$ \\
\hline awt & none & $\begin{array}{l}\text { Atomic weight of the target nuclide. } \\
\text { The program calculates AWRI as AWRI = awt/1.008665. }\end{array}$ & none \\
\hline $\mathrm{zam}$ & none & ZZAAA of the target nuclide & none \\
\hline spin & none & Spin of the target nuclide & none \\
\hline mat & none & Material number of the target & none \\
\hline inflag & none & $\begin{array}{l}1 \text { or } 2 \text {. } \\
\text { The program will read inflag }{ }^{*} \Gamma_{\mathrm{n}} \text { from the BNL325.TXT. } \\
\text { Of usual, inflag }=2 \text { for odd and }=1 \text { for even nuclide. }\end{array}$ & none \\
\hline sfo & $1 \mathrm{E}-04$ & $\begin{array}{l}\text { s-wave neutron strength function for the Bayesian analysis. The s- } \\
\text { wave strength function is obtained from the fitting of reduced } \\
\text { neutron widths. Since the fitted value may be different from this } \\
\text { input value, it is recommended to re-run PTANAL with fitted } \\
\text { strength function. Note, however, that the s-wave probability is not } \\
\text { so sensitive to the strength function. }\end{array}$ & 0. \\
\hline $\begin{array}{l}\text { sfl(1), } \\
\text { sfl(2), } \\
\text { sfl(3) }\end{array}$ & $1 \mathrm{E}-04$ & $\begin{array}{l}\text { A set of three trial p-wave neutron strength functions. } \\
\text { The p-wave probability is calculated with each sfl. }\end{array}$ & 0. \\
\hline $\mathbf{s f 2}$ & 1E-04 & d-wave neutron strength function for the Bayesian analysis. & 0. \\
\hline iset & none & $\begin{array}{l}\text { Index number selected among sfl values. }(1,2, \text { or } 3) \\
\text { The 'sfl(iset)' value is used for final decision making from the } \\
\text { Bayesian p-wave probability. }\end{array}$ & 1 \\
\hline Do & $\mathrm{eV}$ & Average spacing of s-wave resonances for the Bayesian analysis & $\mathbf{0 .}$ \\
\hline ecut & $\mathrm{eV}$ & $\begin{array}{l}\text { Upper energy limit for the analysis. } \\
\text { Recommended: Energy of the last resonance }+2 * \Gamma\end{array}$ & 100000 \\
\hline $\begin{array}{l}\text { ggavg(1) } \\
\text { ggavg(2) }\end{array}$ & $\mathrm{meV}$ & $\begin{array}{l}\text { Average gamma width for } s \text { - and p-wave, respectively. These values } \\
\text { are assigned to resonances with unknown gamma widths. }\end{array}$ & 0. \\
\hline $\begin{array}{l}\operatorname{gncut}(1) \\
\operatorname{gncut}(2)\end{array}$ & $\mathrm{meV}$ & $\begin{array}{l}\text { Weak resonance cutoff reduced width for } s-\text { and p-wave, } \\
\text { respectively. Resonances having widths smaller than the values are } \\
\text { excluded from the fitting process. }\end{array}$ & 0. \\
\hline gncuth & $\mathrm{meV}$ & $\begin{array}{l}\text { Strong resonance cutoff reduced width for } s-\text { wave. Resonances } \\
\text { having widths larger than this value are excluded from the fitting } \\
\text { process. Large doublet or triplet resonances may be excluded from } \\
\text { the analysis by using this feature. }\end{array}$ & 10000 \\
\hline uncert & $\%$ & $\begin{array}{l}\text { Default uncertainty of } \mathrm{g} \Gamma_{\mathrm{n}} \text { of which uncertainty is not given in } \\
\text { BNL325.TXT file. If 'uncert' is less than zero, its absolute value } \\
\text { overrides all the uncertainties given in the BNL325.TXT. }\end{array}$ & 10 \\
\hline disp & none & $\begin{array}{l}\text { Spin dispersion parameter; } \sigma \text {, for the Bethe formula } \\
\text { Note: with a large 'disp', the level density follows }(2 \mathrm{~J}+1) \text { law. }\end{array}$ & 100 \\
\hline \multicolumn{4}{|c|}{ Sample input (for Dy-160) } \\
\hline \multicolumn{4}{|c|}{$\begin{array}{l}\text { \&data } \\
\text { awt }=159.925, \text { zam }=66160, \text { spin }=0, \text { mat }=6637, \text { inflag }=1, \text { ecut }=1994.5 \\
\text { sf }=2.0, \mathrm{sfl}=0.7,1.4,2.0, \mathrm{sf} 2=2.2, \mathrm{D} 0=27.3 \\
\text { gncut }=0.0,0.0, \text { gncuth }=100 ., \text { ggavg }=2 * 105.8, \text { uncert }=20 ., \text { disp }=3 . \\
\text { \&end }\end{array}$} \\
\hline
\end{tabular}


Table B2. CFMTA Format (for each resonance)

\begin{tabular}{|c|c|c|c|}
\hline $\begin{array}{l}\text { Column } \\
\text { No. }\end{array}$ & Format & Description & Note \\
\hline \multicolumn{4}{|l|}{ Record 1} \\
\hline $1 \sim 7$ & 17 & ZZAAA & \\
\hline $8 \sim 9$ & A2 & 'RP' & \\
\hline $\begin{array}{l}10 \sim 18 \\
19 \sim 24 \\
25\end{array}$ & $\begin{array}{l}\text { E9.4 } \\
\text { E6.2 } \\
\text { Al }\end{array}$ & $\begin{array}{l}\text { Resonance energy }[\mathrm{eV}] \\
\text { Uncertainty }[\mathrm{eV}] \text {, and } \\
\text { Flag indicating the status of the energy }\end{array}$ & 'R' \\
\hline 26 & $\mathrm{Al}$ & Parity & \\
\hline $\begin{array}{l}27 \sim 29 \\
30 \\
\end{array}$ & $\begin{array}{l}\text { F3.1 } \\
\mathrm{Al}\end{array}$ & $\begin{array}{l}\text { Resonance spin, } \mathrm{J} \text {, and } \\
\text { Flag indicating the status of given } \mathrm{J}\end{array}$ & Of usual 'F' or ' '(blank) \\
\hline $\begin{array}{l}31 \\
32 \\
\end{array}$ & $\begin{array}{l}\mathrm{Il} \\
\mathrm{Al} \\
\end{array}$ & $\begin{array}{l}\text { Angular momentum, } l \text {, and } \\
\text { Flag indicating the status of given } l\end{array}$ & Of usual 'F' or ", \\
\hline $\begin{array}{l}33 \sim 40 \\
41 \sim 47 \\
48\end{array}$ & $\begin{array}{l}\text { E8.4 } \\
\text { E7.4 } \\
\text { Al }\end{array}$ & $\begin{array}{l}\text { Total width } \Gamma[\mathrm{eV}] \text {, } \\
\text { Uncertainty }[\mathrm{eV}] \text {, and } \\
\text { Flag indicating the status of given value }\end{array}$ & Of usual, not provided \\
\hline $\begin{array}{l}49 \sim 56 \\
57 \sim 63 \\
64\end{array}$ & $\begin{array}{l}\text { E8.4 } \\
\text { E7.4 } \\
\mathrm{Al}\end{array}$ & $\begin{array}{l}\mathrm{g} \Gamma_{\mathrm{n}} \text { or } 2 \mathrm{~g} \Gamma_{\mathrm{n}}[\mathrm{eV}] \\
\text { Uncertainty [eV], and } \\
\text { Flag indicating the status of given value }\end{array}$ & See 'inflag' in PTANAL.INP \\
\hline $\begin{array}{l}65 \sim 72 \\
73 \sim 79 \\
80 \\
\end{array}$ & $\begin{array}{l}\text { E8.4 } \\
\text { E7.4 } \\
\text { A1 }\end{array}$ & $\begin{array}{l}\text { Radiative width } \Gamma_{\gamma}[\mathrm{eV}] \\
\text { Uncertainty }[\mathrm{eV}] \text {, and } \\
\text { Flag indicating the status of given value }\end{array}$ & $\begin{array}{l}\text { PTANAL accepts this value even } \\
\text { if the flag is ' } A \text { '. }\end{array}$ \\
\hline $\begin{array}{l}81 \sim 88 \\
89 \sim 95 \\
96 \\
\end{array}$ & $\begin{array}{l}\text { E8.4 } \\
\text { E7.4 } \\
\text { A1 }\end{array}$ & $\begin{array}{l}\text { s-wrave reduced width } \mathrm{g} \Gamma_{\mathrm{n}}^{0} \text { or } 2 \mathrm{~g} \Gamma_{\mathrm{n}}^{0}[\mathrm{eV}] \\
\text { Uncertainty }[\mathrm{eV}] \text {, and } \\
\text { Flag indicating the status of given value }\end{array}$ & Not used in PTANAL \\
\hline $\begin{array}{l}97 \sim 104 \\
105 \sim 111 \\
112\end{array}$ & $\begin{array}{l}\text { E8.4 } \\
\text { E7.4 } \\
\text { A1 }\end{array}$ & $\begin{array}{l}\text { Miscellaneous quantity, } \\
\text { Uncertainty, and } \\
\text { Flag indicating the status of given value }\end{array}$ & $\begin{array}{l}\text { Of usual, capture area } \mathrm{g} \Gamma_{\mathrm{n}} \Gamma_{\gamma} / \Gamma \\
{[\mathrm{eV}] \text { is provided. In this case, if }} \\
\mathrm{g} \Gamma_{\mathrm{n}} \text { is not provided, PTANAL } \\
\text { calculates } \mathrm{g} \Gamma_{\mathrm{n}} \text { from this quantity } \\
\text { with assumed } \mathrm{g} \text { and } \Gamma_{Y .}\end{array}$ \\
\hline $113 \sim 115$ & A3 & 'BNL' & \\
\hline $116 \sim 119$ & $\mathrm{~A} 4$ & Date in 'YYMM' & \\
\hline $120 \sim 124$ & 15 & $' 10000 '$ & \\
\hline $125 \sim 132$ & & Parameter codes & \\
\hline \multicolumn{4}{|l|}{ Record 2} \\
\hline $1 \sim 7$ & & Same as Record 1 & \\
\hline $8 \sim 9$ & $\mathrm{~A} 2$ & 'R2' & \\
\hline $10 \sim 25$ & & Same as Record 1 & \\
\hline $26 \sim 32$ & & Blanks & \\
\hline $33 \sim 112$ & & Reserved for 5 additional quantities & \\
\hline $113 \sim 124$ & & Same as Record 1 & \\
\hline
\end{tabular}




\section{Appendix C: WRIURR User's Guide}

The WRIURR program appends the average resonance parameters for the unresolved resonance region to the end of the resolved region data in File 2 of ENDF- 6 format.

The program requires two input files, WRIURR.INP and ENDFR.TXT. The ENDFR.TXT file contains resolved resonance parameters in ENDF-6 format. The ENDFR.TXT file is the same as the ENDFA.TXT file, except that it contains bound level resonance parameters, if any.

The WRIURR.INP file is the standard input file in a name-list format. This file consists of :

First card: "\&urr" from column 2 to 6 (Not '\&data' as in PTANAL);

In the second to (last-1) cards: a data section with the name-list keywords; and Last card: "\&end" from column 2 to 5.

The data section includes variables shown in Table $\mathrm{Cl}$.

WRIURR.LIS and ENDFU.TXT are the output files. The former one is the standard output summarizing the input information and calculated results of level spacing. ENDFU.TXT contains Files 1 and 2: the File 2 contains complete resonance parameter information in both the resolved and unresolved energy regions. One may edit the File 1 (general description) manually. 
Table C1. Variables appearing in the WRIURR.INP file

\begin{tabular}{|c|c|c|}
\hline Name & Unit & Description \\
\hline $\begin{array}{l}\text { sf(1) } \\
\text { sf(2) } \\
\text { sf(3) }\end{array}$ & $1 E-04$ & Neutron strength functions of s-, p-, and d-wave, respectively \\
\hline $\begin{array}{l}\operatorname{gg}(1) \\
g g(2) \\
g g(3)\end{array}$ & meV & Average gamma widths of s-, p-, and d-wave, respectively \\
\hline $\begin{array}{l}\mathrm{ds} O(1) \\
\mathrm{ds} O(2) \\
\mathrm{ds} 0(3)\end{array}$ & $\mathrm{eV}$ & $\begin{array}{l}\text { Average level spacing of } \mathrm{s}^{-}, \mathrm{p}^{-} \text {, and d-wave, respectively } \\
\text { Normally only ds } 0(1) \text { is required then ds } 0(2) \text { and ds } 0(3) \text { are calculated in the } \\
\text { program using the Bethe formula. If ds } 0(2) \text { and ds } 0(3) \text { are provided, energy- } \\
\text { independent level spacing and } \Gamma_{\mathrm{n}}^{\prime} \text { are written in ENDFU.TXT. }\end{array}$ \\
\hline $\mathrm{e} 0$ & $\mathrm{eV}$ & Low energy boundary of the unresolved resonance region \\
\hline $\begin{array}{l}\text { de(i) } \\
i=1 \text { to up } \\
\text { to } 300\end{array}$ & $\mathrm{eV}$ & $\begin{array}{l}\text { Energy intervals } \\
\text { The energy points that will be written in File } 2 \text { are calculated as } \mathrm{e} 0 \text { (first energy } \\
\text { point), } \mathrm{e} 0+\mathrm{de}(1), \mathrm{e} 0+\operatorname{de}(1)+\mathrm{de}(2), \text { and so on. }\end{array}$ \\
\hline bn & $\mathrm{MeV}$ & Neutron binding energy of the target nuclide \\
\hline pair & $\mathrm{MeV}$ & Pairing energy of the target nuclide \\
\hline dena & $\mathrm{MeV}^{-1}$ & Level density parameter of the target nuclide \\
\hline disp & none & Spin dispersion parameter, $\sigma$, of the target nuclide \\
\hline $\begin{array}{l}i \operatorname{con}(1) \\
i \operatorname{con}(2) \\
i \operatorname{con}(3)\end{array}$ & none & $\begin{array}{l}\text { Flags indicating the energy dependence of level spacing and } \Gamma_{\mathrm{n}}^{l} \text { for } \mathrm{s}-, \mathrm{p}-\text {, and } \mathrm{d}- \\
\text { wave, respectively. If it is } \mathrm{l} \text {, the program writes energy-dependent level spacing } \\
\text { and recluced width using the Bethe formula. Otherwise, the program writes } \\
\text { energy.independent values. Note that the strength functions are energy-- } \\
\text { independent in any case. Parameters such as 'bn', 'pair', 'dena', and 'disp' are } \\
\text { used in the calculation of energy-dependent level spacings. }\end{array}$ \\
\hline & & Sample input (for Eu-153) \\
\hline \multicolumn{3}{|c|}{$\begin{array}{l}\text { \&urr } \\
\text { bn }=6.435, \text { pair }=0 ., \text { dena }=20.00, \text { icon }=3 * 1, s f=2.37,0.6,5.4, \mathrm{gg}=3 * 93 ., \mathrm{ds} 0=1.14 \\
\mathrm{e} 0=97.8, \mathrm{de}=402.2,500 ., 1000 ., 4^{*} 2000 ., 7^{*} 10000 ., 3910 . \\
\text { \&end }\end{array}$} \\
\hline
\end{tabular}


